\title{
La Movilidad Socioeconómica Intergeneracional en Argentina
}

\author{
Maribel Jiménez \\ Tesis Doctoral
}

Doctorado en Economía

Facultad de Ciencias Económicas

Universidad Nacional de La Plata

Director de Tesis: Dr. Leonardo Gasparini

Director de Tesis Asociado: Dr. Jorge A. Paz

La Plata, 1 de marzo, 2016 


\section{Índice general}

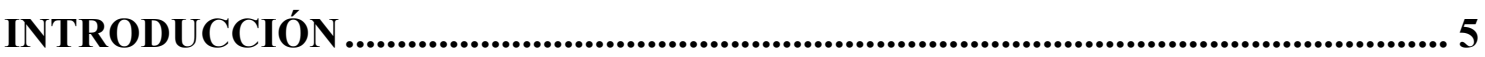

CAPÍTULO 1: MOVILIDAD EDUCATIVA INTERGENERACIONAL EN ARGENTINA. UN ANÁLISIS DE SU EVOLUCIÓN DESDE EL ENFOQUE DE IGUALDAD DE OPORTUNIDADES................................................................... 17

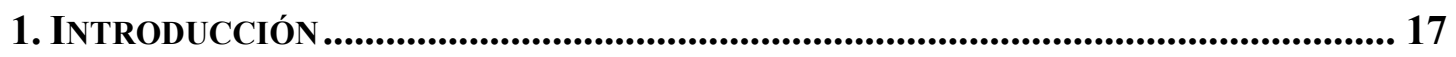

2. LA EVOLUCIÓN DE LA MOVILIDAD EDUCATIVA INTERGENERACIONAL: REVISIÓN

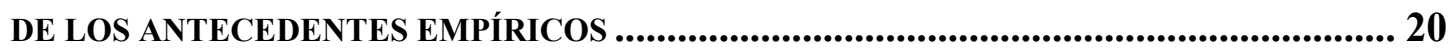

3. MOVILIDAD INTERGENERACIONAL E IGUALDAD DE OPORTUNIDADES ................... 30

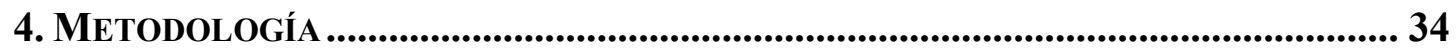

4.1. Enfoque paramétrico: los modelos de regresión intergeneracional................ 35

4.2. Enfoque no paramétrico: matrices de transición y la técnica del overlap ....... 39

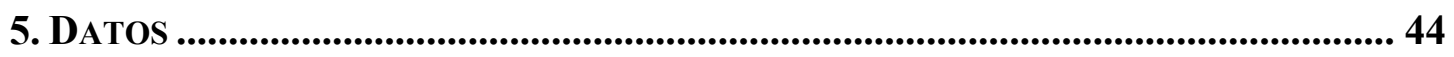

6. RESULTADOS................................................................................................................ 47

6.1. El modelo de regresión intergeneracional y los cambios en la correlación educativa entre generaciones ............................................................................ 47

6.2. Comparaciones temporales de los patrones de movilidad intergeneracional a partir de matrices de transición ...................................................................................... 52

6.3. Evaluación de los procesos de movilidad educativa intergeneracional desde el enfoque de igualdad de oportunidades ........................................................... 58

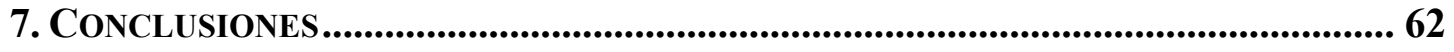

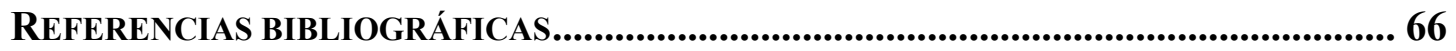

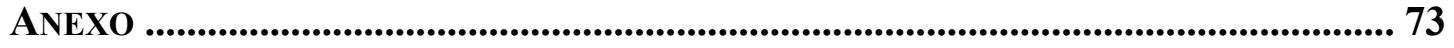

CAPÍTULO 2: MOVILIDAD INTERGENERACIONAL DEL INGRESO EN ARGENTINA: EVALUANDO MÉTODOS Y COMPARANDO RESULTADOS

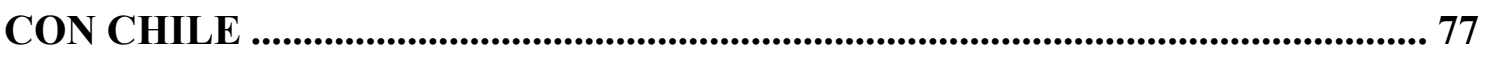

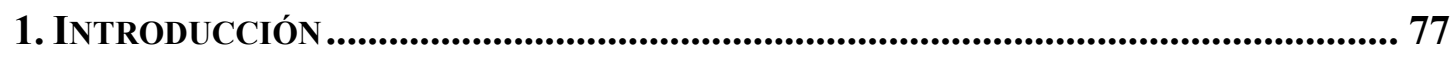

2. LA MOVILIDAD INTERGENERACIONAL DEL INGRESO: UNA REVISIÓN DE LOS

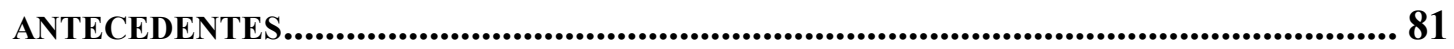

2.1. La medición de la MII en los estudios empíricos .............................................. 81 


\section{ANÁLISIS DE LOS MODELOS EMPÍRICOS INTERGENERACIONALES DEL INGRESO Y}

3.1. El modelo intergeneracional de ingreso permanente y el modelo clásico de error de medición

3.2. El método de estimación con variables instrumentales utilizando dos muestras

3.3. El efecto del life-cycle bias......

3.4. Un modelo intergeneracional alternativo y un nuevo método de estimación 107

3.5. ¿Qué medida de ingreso utilizar?

4. DAtos

5. RESUltados.

5.1. Estimaciones del modelo intergeneracional del ingreso permanente: una primera comparación entre Argentina y Chile

5.2. Cambios temporales en la persistencia intergeneracional del ingreso en Argentina.

5.3. Evaluación del nuevo modelo intergeneracional y su método de estimación 133

6. CONCLUSIONES................................................................................................................... 137

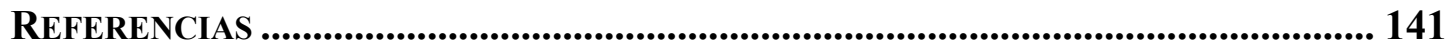

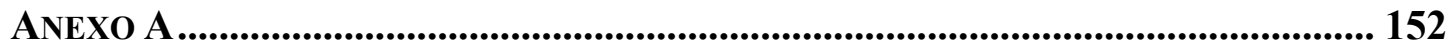

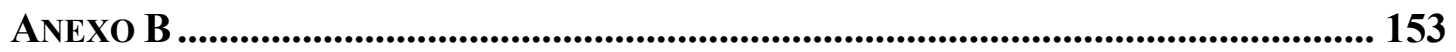

CAPÍTULO 3: MOVILIDAD INTERGENERACIONAL Y GASTO PÚBLICO. EVIDENCIA PARA ARGENTINA ........................................................................... 166

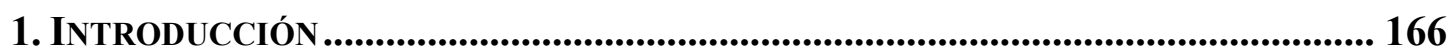

2. MODELOS TEÓRICOS Y ANTECEDENTES EMPÍRICOS ........................................ 169

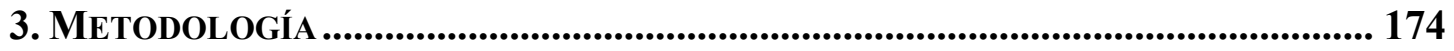

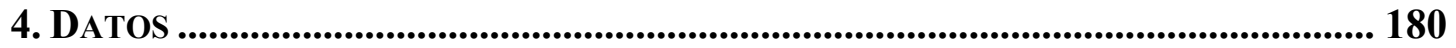

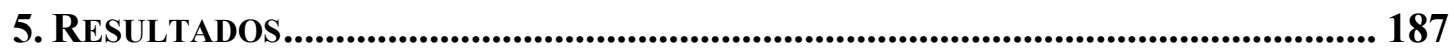

5.1. Efecto de distintas medidas de inversión pública en capital humano en la movilidad intergeneracional

5.2. Brechas de movilidad y gasto público: resultados del método de diferencias en diferencias. 195 
5.3. Las distintas categorías del gasto público y sus efectos en la movilidad

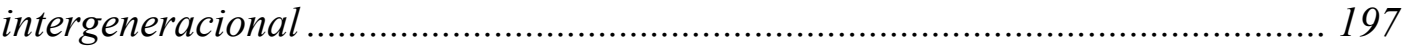

5.4. Gasto público y movilidad intergeneracional entre estratos de ingresos...... 203

5.5. Análisis de robustez ................................................................................... 207

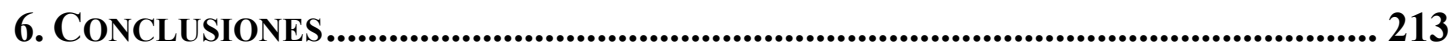

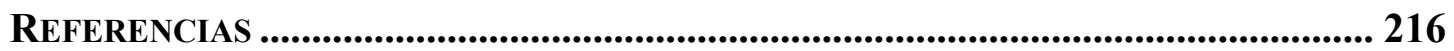

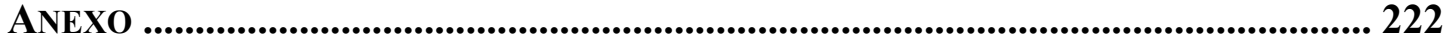




\section{Introducción}

El objetivo general de esta tesis es analizar la movilidad socioeconómica intergeneracional (MI) en Argentina. Éste es un concepto que se encuentra aún en desarrollo y es definido en la literatura con mucho menos claridad que el concepto de desigualdad (Benabou y Ok, 2001a y Formby et al. 2004). No obstante, siguiendo a Behrman (2000), se puede definir la movilidad social, sin pérdida de generalidad, como el movimiento de indicadores de status socioeconómico para entidades específicas entre período de tiempo. El estudio de la movilidad social tiene, entonces, diferentes facetas según cómo se mida la posición económica (a partir de la educación, los ingresos, el consumo, la riqueza o la clase social, por ejemplo), cuál sea el período temporal considerado (corto o largo plazo) y qué unidades de análisis se incluyan (individuos o familias). Desde el punto de vista del alcance temporal, si lo que interesa analizar son los cambios de corto o mediano plazo en la posición económica de una misma persona entonces se habla de movilidad intrageneracional. En cambio, en un estudio de movilidad intergeneracional el foco se extiende a períodos más extensos en los que se evalúan cambios entre generaciones. La MI se refiere, entonces, a la relación que existe entre el nivel socioeconómico de padres e hijos, medido generalmente a través de su educación o de sus ingresos (Conconi et al., 2008).

La movilidad así entendida agrega una dimensión dinámica fundamental al análisis distributivo pues permite determinar en qué medida la posición económica actual está determinada por la del pasado. Por esto, el origen socioeconómico de los individuos debería ser tenido en cuenta cuando se compara su situación presente. En esta línea, Becker (1987) afirma que un estudio completo de la distribución del ingreso debería incluir tanto la desigualdad entre familias en la misma generación, como la desigualdad entre generaciones diferentes de la misma familia, denominada movilidad social intergeneracional. Justamente, en su modelo teórico, Becker y Tomes (1979, 1986) se propusieron integrar la teoría de la distribución del ingreso (diferencias intrageneracionales) con la teoría de la movilidad (diferencias intergeneracionales) en base al supuesto del comportamiento maximizador de la utilidad. Esto nos conduce, 
entonces, a un concepto intergeneracional de desigualdad (Atkinson y Bourguignon, 2000).

Asimismo, como señala Stokey (1998) dos sociedades con distribución idéntica pero diferentes regímenes de movilidad no son equivalentemente igualitarias. Desde esta perspectiva, un escenario de alta movilidad social acompañada de una elevada desigualdad no es considerado tan perjudicial como uno de alta desigualdad combinada con una baja movilidad social (Cruces, 2008).

Además, existe una estrecha relación entre desigualdad y movilidad intergeneracional. De acuerdo con el modelo de Solon (2004), que constituye una interesante extensión teórica del modelo formulado por Becker y Tomes $(1979,1986)$, los mismos factores que afectan positivamente la persistencia intergeneracional de ingresos, incrementan la desigualdad cross-section del ingreso. Estos factores son una mayor productividad de las inversiones en capital humano de los hijos $(\mathrm{CH})$, una menor progresividad de la inversión pública en $\mathrm{CH}$, retornos crecientes al $\mathrm{CH}$ y un mayor nivel de heredabilidad de las dotaciones familiares ${ }^{1}$. Justamente, estos son los principales canales a través de los cuales los ingresos se transmiten intergeneracionalmente.

Asimismo, las percepciones de movilidad socioeconómica por parte de la sociedad pueden afectar las preferencias por la redistribución, condicionando la tendencia de largo plazo de las políticas públicas (Cruces, 2008). Estos argumentos han sido formalizados por Benabou y Ok (2001b) y por Benabou y Tirole (2006) en modelos teóricos que muestra cómo mayores oportunidades engendran más tolerancia por la desigualdad. Si las personas perciben que la sociedad es más justa porque existe una mayor movilidad y el éxito económico es altamente dependiente de su esfuerzo, esto puede tener implicancias para las políticas redistributivas en la medida en que mayores votantes con esta visión terminen representando un bloque votante crucial. Por lo tanto, la movilidad social puede potenciar una creciente cohesión social o un mayor apoyo político del sistema.

\footnotetext{
${ }^{1}$ En este modelo se entiende por dotación todo lo que puede ser transmitido de una generación a otra como, por ejemplo, habilidades cognitivas, preferencias, hábitos y formas de conducta, etc.
} 
Por otra parte, las medidas de MI están estrechamente relacionadas con el concepto de igualdad de oportunidades ${ }^{2}$ que permite analizar el grado con el que el origen socioeconómico de los individuos determina su conjunto de oportunidades disponibles. Por esto, la MI, en la medida en que implica una mayor igualdad de oportunidades, puede tener consecuencias importantes para la eficiencia económica (Conconi et al., 2008). Así, la posibilidad de tener movilidad socioeconómica incentiva a los individuos a realizar un mayor esfuerzo para aprovechar sus propias cualidades. Además, la persistencia de la desigualdad de oportunidades puede crear el denominado efecto túnel descripto por Hirschman y Rothschild (1973). De acuerdo con esta hipótesis, los retrocesos de los demás proveen información acerca de un ambiente externo más perverso que impide a las personas mantener la esperanza de ver alguna luz al final del túnel. En cambio, un mayor índice de MI indicaría que el origen socioeconómico de los individuos es menos importante en la determinación de su conjunto de oportunidades disponibles. En este caso, la tolerancia respecto de las desigualdades e injusticias predominará sobre la impaciencia, generándose una especie de "válvula de seguridad" que promueve la cohesión social.

A pesar de las razones previamente mencionadas que justifican el análisis de la movilidad socioeconómica intergeneracional, los estudios empíricos que examinan este tema son relativamente escasos en Argentina. En particular, son prácticamente inexistentes las investigaciones sobre MI del ingreso en el país. Ello probablemente responde a las limitaciones de las fuentes de datos disponibles. El análisis de la MI requiere contar con información sobre características socioeconómicas de padres e hijos para individuos adultos ${ }^{3}$. Esta información puede obtenerse a partir de tres tipos de datos: (a) encuestas a individuos adultos con preguntas retrospectivas sobre sus padres, (b) datos de panel que se extiendan durante un período de tiempo lo suficientemente largo como para incluir información sobre los resultados económicos de dos generaciones y (c) datos administrativos o de registros que permitan vincular la información de los padres con la de sus hijos adultos. Para Argentina y en general, para los países de América Latina, salvo algunas excepciones, no se cuenta con paneles ni de

\footnotetext{
${ }^{2}$ El concepto de igualdad de oportunidades, desarrollado entre otros por Roemer (1998, 2004), ha obtenido un fuerte respaldo del enfoque de capacidades de Sen.

${ }^{3}$ En general, las encuestas de hogares disponibles para Argentina contienen ésta información sólo para los hijos que residen con sus padres y no para todos los individuos entrevistados.
} 
registros administrativos de largo plazo. Sólo en algunos países se dispone de encuestas con información retrospectiva (Torche, 2014). Justamente, éste tipo de datos constituye la principal fuente de información en esta investigación.

La tesis está estructurada en tres capítulos que si bien son auto-contenidos se relacionan y complementan entre sí. Precisamente, los dos primeros capítulos contribuyen al objetivo general de la tesis pues en ellos se estiman y analizan los niveles así como los cambios temporales de la MI en Argentina utilizando dos medidas diferentes del status socioeconómicos de padres e hijos: la educación, en el Capítulo 1 y el ingreso, en Capítulo 2. En tanto que, el tercer capítulo explora los efectos de uno de los principales factores intervinientes en el proceso de transmisión intergeneracional: la inversión pública en el $\mathrm{CH}$ de niños y adolescentes. En concreto, el último capítulo busca determinar en qué medida los niveles y cambios temporales previamente analizados en la movilidad intergeneracional responden a las modificaciones que tuvieron lugar en el gasto público.

Se presenta a continuación un resumen del contenido de cada capítulo a fin de resaltar su contribución a la literatura existente.

Como se mencionó, en el Capítulo 1 se examina la transmisión de la educación entre generaciones. Aunque la literatura sobre este tema ha crecido, la mayoría de las investigaciones existentes examinan la movilidad educativa intergeneracional (MEI) en un determinado punto del tiempo. En cambio, los estudios que intentan medir la MI desde una perspectiva temporal son menos numerosos. Por ello, el primer objetivo de este capítulo es examinar los cambios temporales en la MEI en Argentina, a partir de una base de datos novedosa obtenida de una encuesta de opinión pública denominada Latinobarómetro que se realiza anualmente en varios países de América Latina. La principal ventaja de esta encuesta es la disponibilidad de información retrospectiva sobre la educación de los padres para todos los individuos adultos entrevistados. El análisis temporal se realiza para las cohortes de nacimiento desde 1949 hasta 1988 durante el período 2003-2013.

Por otra parte, como ya se indicó, la MI está relacionada con la noción de igualdad de oportunidades (EOP) pero, a diferencia de lo asumido por la mayoría de los estudios empíricos, no se corresponde directamente con ésta. En particular, los movimientos de la educación (o de los ingresos) entre generaciones pueden ser 
igualadores o desigualadores y los indicadores de movilidad propuestos en la literatura fallan en distinguirlos. Por lo tanto, otro objetivo de este capítulo es determinar las implicancias de los niveles de MI observados para la existencia de EOP así como medir el grado con el cual la MI produce un efecto nivelador de oportunidades. La pregunta central que se intenta responder es en qué medida los cambios observados en el proceso de MI modificaron los niveles de EOP, particularmente entre aquellos que pertenecen a los grupos socioeconómicos más desaventajados. Asimismo, se pretende analizar la forma más adecuada de comparar temporalmente procesos de MI a fin de implementar un criterio robusto para su ordenamiento. Para esto se utiliza una técnica novedosa, recientemente propuesta por Anderson et al. (2014) que consiste en medir el grado de solapamiento (overlap) entre la densidad condicional observada de la educación de los hijos dada la educación de los padres y la densidad teórica correspondiente a una situación de EOP o independencia entre las dos variables. Una de las principales ventaja de este método sobre otros existentes (como el enfoque de dominancia estocástica formulado por Lefranc et al., 2008, 2009) es que ofrece una medida (denominada índice de overlap) que indica el grado de progreso hacia la EOP realizado por una sociedad en su conjunto o por un grupo socioeconómico particular. Éste resulta particularmente útil como estadística para efectuar comparaciones temporales de los procesos de movilidad observados o para evaluar, por ejemplo, qué grupo socioeconómico resultó más beneficiado por una política que busca igualar las oportunidades.

La contribución central de este capítulo a la literatura consiste en evaluar por primera vez para Argentina, los cambios temporales en la MEI desde el enfoque de EOP y en implementar, con este fin, por primera vez para otro país, una técnica novedosa que presenta varias ventajas sobre otros métodos existentes.

Los principales resultados obtenidos muestran una disminución significativa en los niveles promedio de persistencia educativa intergeneracional en Argentina. Sin embargo, se observan también importantes asimetrías en el proceso de MI con una mayor persistencia de la educación entre los padres y los hijos que se encuentran en el nivel educativo más alto. Por otra parte, la evaluación de los procesos de MEI desde el enfoque de EOP, a partir de la técnica de overlap, sugiere una mejora en los niveles promedio de EOP entre las generaciones más jóvenes. No obstante, de acuerdo con los índices $O V$ estimados, esta ganancia en términos de EOP, a lo largo de las cohortes de 
nacimiento, se observa en mayor medida entre los hijos cuyos padres tienen nivel secundario completo y estudios superiores. Más aún, es notable la falta de mejora de los niveles de EOP, medidos por el índice $O V$, entre los hijos de padres con los niveles educativos más bajos

El Capítulo 2 examina la movilidad intergeneracional del ingreso (MII) en Argentina. Los objetivos de este capítulo son varios. En primer lugar, evaluar la performance de distintos estimadores de la elasticidad intergeneracional del ingreso $(\text { EII })^{4}$ que constituye la medida de inmovilidad de ingresos entre generaciones más utilizada en la literatura empírica. Con este fin, se analizan los límites en probabilidad y se evalúan empíricamente los distintos estimadores de la EII disponibles en la literatura, en el contexto del modelo intergeneracional tradicional del ingreso permanente. Para ello resultan particularmente útiles los datos de la Encuesta Panel CASEN 1996-20012006 (EPCASEN) de Chile que constituye uno de los paneles de datos más largo disponible para un país de América Latina. También se emplean los datos de diversas encuestas disponibles para Argentina como la Encuesta de Percepción de planes sociales (EPPS) del 2007, la Encuesta de Desarrollo Social (EDS) de 1997, la Encuesta del International Social Survey Programme (EISSP) de 2010 y la Encuesta Permanente de Hogares (EPH) de varios años. En segundo lugar, el capítulo se propone analizar el nivel así como los cambios temporales de la MII en Argentina. Asimismo, se busca contrastar el grado de persistencia de ingreso entre padres e hijos observado en el país, con el existente en Chile, a partir de estimaciones metodológicamente comparables. Las características comunes y diferentes que presentan estos dos países, susceptibles de tener un efecto importante sobre el proceso de transmisión intergeneracional, hacen que este ejercicio empírico sea particularmente interesante.

En tercer lugar, el capítulo evalúa, por primera vez para otros países (Argentina y Chile, en este caso), un modelo intergeneracional alternativo recientemente propuesto por Muller (2010) y, para ello, se propone un nueva estrategia de estimación. La idea central de este enfoque es que, a diferencia de lo sostenido por el modelo intergeneracional tradicional, la transmisión relevante entre generaciones, no sólo tiene

\footnotetext{
${ }^{4}$ Este indicador de persistencia intergeneracional se obtiene a partir del coeficiente del logaritmo del ingreso del padre en una regresión que tiene como variable dependiente el logaritmo del ingreso de los hijos.
} 
lugar en relación al componente permanente del ingreso sino también, a través de los ingresos transitorios. Esto, a su vez, tiene varias implicancias relevantes. Por un lado, sugiere que las comparaciones realizadas entre países, en base al modelo estándar de transmisión intergeneracional del ingreso permanente, ignoran una fuente potencial de heterogeneidad inobservada que podría afectar las estimaciones de la EII: la edad de los hijos en la que se mide el ingreso de sus padres. Por otra parte, si el ingreso transitorio afecta el ingreso futuro de los niños, esto indicaría que el ingreso de los padres per se tiene un efecto en sus oportunidades, lo que generalmente es incompatible con las formulaciones incluso más débiles del enfoque de EOP. La estimación de este modelo alternativo, al igual que el tradicional, es particularmente problemática en países en los que las bases de datos disponibles no incluyen información sobre el ingreso de los padres para los individuos encuestados, en edades centrales del ciclo vital de ambos. Si bien, una solución ampliamente utilizada para estimar la EII en este caso, es implementar el método Two-Sample Intrumental Variable (TSIV), esta técnica no sería adecuada para el modelo alternativo porque instrumenta el ingreso permanente pero no resuelve el problema de la exclusión del ingreso transitorio del modelo estimado. Por eso, en esta investigación se propone una nuevo enfoque metodológico que combina algunas características del método TSIV, para estimar el componente permanente del ingreso parental y del enfoque propuesto por Dang et al. (2014) que permite estimar su componente transitorio, particularmente durante la niñez y adolescencia de los hijos. Bajo determinados supuestos, con este método podrían obtenerse un límite inferior y superior de la verdadera EII en el contexto del modelo alternativo.

Las contribuciones del capítulo a la literatura existente son varias. En primer lugar, este es el primer estudio que analiza la MII en Argentina, a partir de la información retrospectiva sobre los padres de los entrevistados proveniente de distintas encuestas disponibles (la EDS 1997, la EPPS 2007 y la EISSP 2010) y que obtiene estimaciones metodológicamente comparables con las de otro país latinoamericano (Chile). En segundo lugar, el capítulo ofrece una derivación detallada de los límites en probabilidad de los estimadores de la EII utilizados en la literatura, lo que permite examinar formalmente sus potenciales sesgos. En tercer lugar, se evalúa aquí, por primera vez para otros países (Argentina y Chile), un modelo intergeneracional alternativo y se propone, para ello, una técnica de estimación novedosa. 
Los resultados obtenidos de la estimación del modelo tradicional de ingreso permanente sugieren que los niveles de MII disminuyeron entre 1997 y 2007 y tienden a ser menores en Chile que en Argentina. Sin embargo, cuando se considera el modelo intergeneracional alternativo, las estimaciones obtenidas con el nuevo método, sugieren que, los niveles de MII son similares en ambos países y relativamente bajos. También indican que los ingresos transitorios de los padres explicarían como máximo el 18\% y el $25 \%$ de la correlación del ingreso observada entre padres e hijos en Chile y Argentina, respectivamente. No obstante, estos resultados deben ser interpretados con precaución porque fueron derivados bajo determinados supuestos.

En el Capítulo 3 se evalúa el efecto del gasto público sobre los niveles de movilidad intergeneracional de la educación y de los ingresos previamente observados en el país. En los últimos años, la mayoría de la literatura sobre MI, particularmente, la que analiza la MII se ha concentrado en obtener una medida adecuada del nivel de MI, siendo relativamente más escasas las investigaciones que avanzaron en la identificación de los factores causales subyacentes al proceso de transmisión del ingreso entre generaciones. Según los modelos teóricos que analizan los factores asociados con la MII (Mulligan, 1997; Solon, 2004; Ichino et al., 2011), no sólo la inversión de los padres en sus hijos es uno de los principales canales de transmisión intergeneracional de ingresos, sino también la inversión del gobierno en el capital humano de los niños y adolescentes. Por ello, el principal objetivo de este capítulo es determinar el efecto que tiene sobre la MI en Argentina, el gasto público, concretamente, el realizado por los tres niveles de gobiernos (nacional, provincial y municipal) que pueden modificar las inversiones públicas en el capital humano de los niños y adolescentes. Específicamente, se busca evaluar si el gasto público cumple con uno de los objetivos centrales de la política social que es incrementar la movilidad económica intergeneracional y la EOP, disminuyendo el impacto del origen socioeconómico en la educación o el ingreso obtenido en la adultez. Asimismo, se pretende examinar si la brecha de movilidad observada entre los hijos que provienen de distintos trasfondos socioeconómicos familiares es menor para aquellos que experimentaron mayores niveles de gasto público durante su adolescencia.

Los estudios empíricos que examinan la relación entre MI y gasto público son escasos a nivel internacional y, prácticamente inexistentes, a nivel nacional. Entre los antecedentes más directos de esta investigación se encuentran los estudios de Mayer y 
Lopoo (ML) (2008) para Estados Unidos y Behrman, Gaviria y Székeley (BGS) (2001) para 19 países latinoamericanos y Estados Unidos. A diferencia de estos estudios que se concentran en una medida de posición socioeconómica, el ingreso, en el caso del primero y la educación, en el caso del segundo, en esta investigación se examina el efecto del gasto público tanto sobre la MII como sobre la MEI. Además, a diferencia de los estudios mencionados que sólo analizan el efecto del gasto total (en el caso de ML) o del gasto en educación (en el caso de BGS), en este capítulo se consideran distintas categorías y agregados del gasto público consolidado (GPC), particularmente del gasto público social, con objetivo de explorar el efecto que diferentes medidas de la inversión pública en el capital humano de niños y adolescentes tienen en la MI.

Por otro parte, la estrategia de identificación empleada se basa fundamentalmente en explotar la variabilidad temporal del gasto público, de forma similar a como lo hacen ML y BGS. Sin embargo, a diferencia de estos estudios, en los modelos estimados se incluyen, además de efectos fijos locales, un amplio conjunto de variables de control a nivel local que capturan características variables en el tiempo (o entre cohortes) potencialmente relacionadas con el gasto público y con las variables de resultados (ingresos y educación) como, por ejemplo, la recaudación tributaria provincial y la carga tributaria nacional. La omisión de estas variables de control podrían sesgar las estimaciones del término de interacción entre la posición socioeconómica de los padres y el gasto público que captura el efecto que éste último tiene en las medidas de persistencia intergeneracional consideradas. No obstante, como es probable que, a pesar de los efectos fijos por provincia, por cohorte y por año, de los controles por las características de padres e hijos así como por características socioeconómicas de las provincias incluidas en el modelo, existan factores inobservables omitidos que pueden segar el efecto estimado del gasto público sobre la MI, se implementa otra estrategia de identificación propuesta por ML. Esta técnica consiste en explorar el efecto del gasto público consolidado sobre la MI utilizando un enfoque de diferencias en diferencias. Específicamente, se asume que la "brecha de movilidad" entre hijos que provienen de un trasfondo familiar aventajado y aquellos que no están en esta situación, es menor en las provincias con un elevado gasto público consolidado per cápita que en aquellas con un bajo gasto público. Asimismo, a diferencia de los antecedentes mencionados, a fin de examinar la robustez de los resultados obtenidos ante cambios en la edad y el período temporal en la que se mide la 
inversión pública en el capital humano de los hijos, se utilizan, además del GPC, los datos sobre el gasto público provincial per cápita por finalidad y función para el período 1993-2012.

Los resultados obtenidos, a partir de los datos de la EPH para el período 19982012, sugieren que el gasto público tiene el efecto esperado, incrementando la movilidad intergeneracional tanto del ingreso como la educativa. Sin embargo, no todas las categorías de gasto son igualmente efectivas para conseguir este resultado. Así, se observa una disminución mayor en la persistencia socioeconómica entre generaciones cuando se incrementan las categorías del GPC más directamente relacionadas con el capital humano de los niños y adolescentes: el gasto en salud, nutrición y educación. No obstante, las magnitudes de los efectos estimados son modestas, requiriéndose un elevado incremento del gasto para lograr mejoras significativas en la movilidad intergeneracional. 


\section{Referencias}

Anderson, G., Leo, T. W. y Muelhaupt, R. (2014). "Measuring advances in equality of opportunity: The changing gender gap in educational attainment in Canada in the last half century", Social Indicators Research, 119(1), 73-99.

Atkinson, A. y Bourguignon, F. (2000). "Income distribution and economics", en A. Atkinson y F. Bourguignon, eds., Handbook of Income Distribution. Amsterdam: Elsevier Science, 1-58.

Becker, G. (1987). Tratado sobre la familia. Madrid: Alianza Editorial.

Becker, G. S. y Tomes, N. (1979). “An equilibrium theory of the distribution of income and intergenerational mobility", Journal of Political Economy, 87(6), 1153-89.

Becker, G. S. y Tomes, N. (1986). "Human capital and the rise and fall of families", Journal of Labor Economics, 4(3), pt. 2, S1-S39.

Behrman, J. (2000). "Social mobility: concepts and measurements", en N. Birdsall y C. Graham, eds., New Markets, New Opportunities? Economic and social mobility in a changing world, Washington, DC: Brookings Institution, 69-100.

Behrman, J. R, Gaviria, A. y Székeley, M. (2001). “Intergenerational mobility in Latin America", Economia, 2(1), 1-31.

Benabou, R. y Ok, E. A. (2001a). "Mobility as progressivity: ranking income process according to equality of opportunity", National Bureau of Economic Research, NBER Working paper series No. 8431.

Benabou, R. y Ok, E. A. (2001b). "Social mobility and the demand for redistribution: The POUM hypothesis", The Quarterly Journal of Economics, 116(2), 757-72.

Benabou, R. y Tirole, J. (2006). "Belief in a just World and redistributive politics", The Quarterly Journal of Economics, 121 (2), 699-746.

Conconi, A., Cruces, G., Olivieri, S. y Sánchez, R. (2007). "E pur si muove? movilidad, pobreza y desigualdad en América Latina”, Económica, LIV(1-2), 121-159.

Cruces, G. (2008). "Tópicos de Economía distributiva”, Maestría en Economía, Facultad de Ciencias Económicas, Universidad Nacional de La Plata, Material de clases no publicado.

Dang, H., Lanjouw, P., Luoto, J., McKenzie, D. (2014). "Using repeated cross-sections to explore movements into and out of poverty", Journal of Development Economics, $107,112-128$. 
Formby, J. P., Smith, W. J. y Zheng, B. (2004). "Mobility measurement, transition matrices and statistical inference", Journal of Econometrics, 120(1), 181-205.

Hirschman, A. y Rothschild, M. (1973). "The changing tolerance for income inequality in the course of economic development with a mathematical appendix", The Quarterly Journal of Economics, 87(4), 544-66.

Ichino, A., Karabarbounis, L., Moretti, E. (2011), “The political economy of intergenerational income mobility", Economic Inquiry, 49(1), 47-69.

Lefranc, A., Pistolesi, N. y Trannoy, A. (2008). "Inequality of opportunity vs. Inequality of outcomes: are western societies all alike?", Review of Income and Wealth, 54(4), 513-546.

Lefranc, A., Pistolesi, N., y Trannoy, A. (2009). "Equality of opportunity and luck: definitions and testable conditions, with an application to income in France", Journal of Public Economics, 93(11-12), 1189-1207.

Mayer, S. E. y Lopoo, L. M. (2008). "Government spending and intergeneracional mobility", Journal of Public Ecoomics, 92(1-2), 139-158.

Muller, S. M. (2010). "Another problem in the estimation of intergenerational income mobility”, Economics Letters, 108(3), 291-295.

Mulligan, C. (1997). Parental priorities and economic inequality. Chicago: University of Chicago Press.

Roemer, J. (1998). Equality of opportunity. Cambridge Mass.: Harvard University Press.

Roemer, J. (2004). "Equal opportunity and intergenerational mobility: going beyond intergenerational income transition matrices", en M. Corak, ed., Generational Income mobility in North America and Europe. Cambridge: Cambridge University Press.

Solon, G. (2004). "A model of intergenerational mobility variation over time and place", en M. Corak, ed., Generational income mobility in North America and Europe. Cambridge: Cambridge University Press, 38-47.

Stokey, N.L. (1998). "Shirtsleeves to Shirtsleeves: The economics of social mobility", en Jacobs, D. P., Kalai, E. y Kamien, M. I., eds., Frontiers of research in economic theory: the Nancy L. Schwartz memorial lectures 1983-1997. Cambridge: Cambridge University Press, 210-241.

Torche, F. (2014). "Intergenerational mobility and inequality: The Latin American case", Annual Review of Sociology, 40, 619-642. 


\section{Capítulo 1*}

\section{Movilidad educativa intergeneracional en Argentina. Un análisis de su evolución desde el enfoque de igualdad de oportunidades.}

\section{Introducción}

La educación juega un doble papel en el proceso de transmisión intergeneracional del status socioeconómico. Puede ser uno de los principales canales de movilidad social pero también puede ser una vía para la reproducción de las desigualdades. El alcance de la educación para promover la movilidad depende del efecto que el entorno familiar de origen tiene sobre el logro educativo de los hijos (Torche, 2010). En particular, depende del efecto de la educación de los padres sobre la educación de los hijos, es decir, del nivel de movilidad educativa intergeneracional (MEI) que exista en una sociedad.

El análisis de transmisión de la educación entre generaciones es un punto de partida para comprender mejor los factores explicativos claves de las relaciones intergeneracionales en otras variables relevantes como el ingreso. En efecto, los logros educativos constituyen una buena medida proxy de la dotación de capital humano de los individuos que, a su vez, está positivamente correlaciona con el ingreso permanente (Checchi et al., 2008) $)^{5}$. Además, existe una relación estrecha entre la MEI y la movilidad intergeneracional del ingreso. En un estudio reciente, Blanden (2013) deriva

\footnotetext{
- Agradezco los comentarios de Leonardo Gasparini, Jorge Paz y los realizados por el Comité de Doctorado a versiones previas de este capítulo.

5 Además los datos sobre logros educativos, particularmente los referidos a los padres de los individuos entrevistados, están sujetos a menores errores de medición que el ingreso.
} 
formalmente las relaciones entre los dos indicadores más utilizados para medir persistencia intergeneracional de la educación y de los ingresos: el coeficiente de correlación educativa y la elasticidad intergeneracional de ingresos. Desde el punto de vista empírico, hay evidencia que muestra la relación entre disminuciones en la movilidad intergeneracional de ingresos y los incrementos en la persistencia educativa entre generaciones (Blanden et al., 2007).

Aunque la literatura sobre la transmisión de la educación entre padres e hijos ha crecido, la mayoría de las investigaciones existentes examinan la MEI en un determinado punto del tiempo. Los estudios que intentan medir la movilidad intergeneracional (MI) desde una perspectiva temporal son menos numerosos. Sin embargo, este análisis puede llevar a una comprensión más profunda de los mecanismos subyacentes a la transmisión del ingreso entre generaciones (Aaronson y Mazumder, 2008). Además, el análisis dinámico de la MI permite indagar si la elevada o baja inmovilidad entre generaciones observada en un determinado país es un fenómeno relativamente reciente o una característica temporalmente persistente.

Por ello, el primer objetivo de este capítulo es examinar los cambios temporales en la MEI en Argentina a partir de una base de datos novedosa obtenida de una encuesta de opinión pública denominada Latinobarómetro que se realiza anualmente en varios países de América Latina. La principal ventaja de esta encuesta es la disponibilidad de información retrospectiva sobre la educación de los padres para todos los individuos adultos encuestados. Dada la ausencia de datos adecuados para analizar las tendencias en la movilidad intergeneracional del ingreso en Argentina, se considera que la educación ofrece una variable socioeconómica apropiada para examinar la evolución de la movilidad social intergeneracional entre cohortes de nacimiento.

Por otra parte, como se mencionó en la Introducción, el concepto de movilidad se encuentra aún en desarrollo y como resultado, existe menos consenso sobre la forma de medir la movilidad en relación con la medición de la desigualdad (Benabou y Ok, 2001 y Formby et al., 2004). Más importante aún es la falta de consenso sobre la forma de evaluar los procesos de movilidad en los no tan numerosos casos en que se analiza explícitamente esta relevante cuestión. Siguiendo a Benabou y Ok (2001), en esta 
investigación se considera que la igualdad de oportunidades ${ }^{6}$ (EOP, por sus siglas en inglés) ofrece un enfoque muy natural para evaluar el proceso de movilidad intergeneracional. En este contexto, la importancia de la existencia de movilidad de ingreso entre generaciones no radica en que los movimientos de ingresos entre padres e hijos sean intrínsecamente valiosos sino que se espera ayuden a disminuir los efectos negativos de las disparidades de dotaciones iniciales en los ingresos futuros o esperados (Stokey, 1998).

Son diversas las razones que justifican el análisis de la relación entre la MI y el concepto de EOP. Así, el grado de igualdad de oportunidades económicas y sociales establece en qué medida la circunstancias de una persona en su infancia afectan su resultado futuro o, de manera inversa, indica en qué medida los individuos pueden obtener un cierto resultado gracias a su propio talento, motivación y esfuerzo (Blanden et al., 2005). Por esto, la movilidad intergeneracional puede tener consecuencias importantes para la eficiencia económica (Conconi et al., 2008). Una mayor MI, en la medida en que implica la existencia de más igualdad de oportunidades, promueve una asignación más eficiente de las habilidades y ventajas comparativas potenciales de los individuos porque permite una asignación más óptima de los individuos en función de sus capacidades en actividades conformes con sus ventajas comparativas. Por otra parte, la desigualdad económica percibida como desigualdad de oportunidades es, probablemente, una de las principales fuentes de descontento e inestabilidad social y política. Esto está relacionado con el denominado efecto túnel de Hirschman y Rothschild (1973) al que se hizo referencia en la Introducción y que ilustra la tolerancia a la desigualdad en diferentes contextos de movilidad.

Ahora bien, en general, las medidas de MI están relacionadas con la noción de EOP pero no se corresponden directamente con ésta. En particular, los movimientos de ingreso entre generaciones pueden ser igualadores o desigualadores y los indicadores de movilidad propuestos en la literatura fallan en distinguirlos.

Por lo tanto, otro objetivo de esta investigación es identificar así como implementar un método adecuado que permita determinar las implicancias de los

\footnotetext{
${ }^{6}$ El enfoque de igualdad de oportunidades, desarrollado entre otros por Roemer $(1998,2004)$, ha obtenido un fuerte respaldo del enfoque de capacidades de Sen. Sin embargo, algunos como Dubet (2011) han cuestionado el principio de justicia subyacente a este enfoque y principalmente, su prioridad frente a la igualdad de resultados en la agenda política.
} 
niveles de movilidad intergeneracional observados para la existencia de igualdad de oportunidades así como medir el grado con el cual la movilidad intergeneracional produce un efecto nivelador de oportunidades. Asimismo, se pretende analizar la forma más adecuada de comparar temporalmente procesos de movilidad intergeneracional a fin de implementar un criterio robusto para su ordenamiento. Para esto se utiliza una técnica novedosa, recientemente propuesta por Anderson et al (2014) que consiste en medir el grado de solapamiento (overlap) entre la densidad condicional de la educación de los hijos dada la educación de los padres y la densidad teórica correspondiente a una situación de EOP o independencia entre las dos variables. Una de las principales ventaja de este método sobre otros existentes (como el enfoque de dominancia estocástica formulado por Lefranc et al., 2008, 2009) es que ofrece una medida (denominada índice de overlap) que indica el grado de progreso hacia la igualdad de oportunidades realizado por una sociedad en su conjunto o por un grupo socioeconómico particular. Este resulta particularmente útil como estadística para realizar comparaciones temporales de los procesos de movilidad observados o para evaluar, por ejemplo, qué grupo socioeconómico resultó más beneficiado por una política que busca igualar las oportunidades.

El trabajo se estructura de la siguiente manera. En la próxima sección se revisan los estudios sobre MEI, especialmente aquellos que examinan su evolución temporal y los desarrollados para Argentina. En la tercera sección se analizan las relaciones entre movilidad intergeneracional y el concepto de igualdad de oportunidades. En este contexto se destacan las contribuciones de esta investigación a la literatura existente. En la cuarta sección, se presentan los métodos implementados para cumplir con los objetivos del estudio. A continuación, se describen los datos utilizados para el análisis. En la siguiente sección se presentan y analizan los resultados obtenidos. Por último, se enuncian las principales conclusiones del estudio.

\section{La evolución de la movilidad educativa intergeneracional: revisión de los antecedentes empíricos}

La transmisión intergeneracional de la educación ha sido ampliamente estudiada por economistas y sociólogos. Una gran parte de la investigación sobre MEI se ha 
concentrado en examinar el vínculo y grado de asociación entre la educación de padres e hijos. Con este fin, se computa, generalmente, como indicador de inmovilidad educativa intergeneracional el coeficiente de regresión de la educación del padre, en un modelo que tiene como variable dependiente la educación de los hijos o coeficiente de persistencia educativa (CPE). Además de este indicador, también suele computarse el coeficiente de correlación educativa intergeneracional (CCE) que elimina de la medida anterior la dispersión cross-section de la educación en las dos generaciones. De esta manera, si el desvío estándar de la educación es menor en la generación de los padres que en la de los hijos, el CPE será mayor que el CCE. Asimismo, como la educación es medida, generalmente, como una variable discreta, varios estudios suelen emplear métodos que toman en cuenta este hecho como, por ejemplo, modelos probit o logit ordenados. Otra forma ampliamente utilizada en la literatura para caracterizar los procesos de MEI es a partir de matrices de transición (Black y Deveraux, 2011).

Por otra parte, hace tan sólo una década, una parte de la investigación sobre MEI comenzó a concentrarse en la estimación del efecto causal de la educación de los padres sobre los logros educativos de los hijos. Este análisis permite comprender mejor qué conjunto de factores son más relevantes en el proceso de transmisión intergeneracional, si aquellos relacionados con el entorno de la crianza (nurture effect) o con las dotaciones genéticas (nature effect). Los estudios utilizaron tres estrategias de identificación: información sobre gemelos idénticos (Bingley et al., 2009, Haegeland et al., 2010, Pronzato, 2012, entre otros), sobre hijos adoptados (Plug, 2004, Björklund et al., 2006, Sacerdote, 2007, entre otros) y el método de variables instrumentales, utilizando generalmente como instrumento las reformas educativas (Oreopoulos et al., 2006, Maurin y McNally, 2008, Carneiro et al., 2013, entre otros). Holmlund et al. (2011) desarrollan una completa revisión de esta literatura. A partir de ella, los autores concluyen que la educación de los padres representa una gran parte del denominado nurture effect, sin embargo, su efecto causal es relativamente pequeño.

Los antecedentes más relacionados con el objetivo de este capítulo son los estudios que examinan cambios temporales en la MEI. Éstos son relativamente recientes 
y menos numerosos ${ }^{7}$. Una de las investigaciones más amplia, por la cantidad de países considerados, es la realizada por Hertz et al. (2007) que extiende el análisis de la MEI a 42 países. Los autores encuentran que la transmisión de la educación entre generaciones, medida por el CPE, cayó en los últimos 50 años mientras que el CCE permaneció relativamente estable. También observan una considerable variación geográfica en la MEI, con los países nórdicos mostrando los menores niveles de persistencia intergeneracional y los países de América Latina presentando los mayores valores (Ranasinghe, 2015).

Los estudios que tienen como principal objetivo examinar las tendencias en la MEI son más numerosos para los países desarrollados. La evidencia empírica obtenida por estas investigaciones es bastante mixta. Así, Heineck y Riphan (2007) investigan la evolución de la MEI en Alemania, para las cohortes de nacimiento de 1929 a 1978 y no encuentran una reducción significativa en los efectos de la educación de los padres sobre el logro educativo de sus hijos. En su estudio empírico, Hanslin et al. (2007) examinan las tendencias en la educación y su transmisión intergeneracional en Suiza entre dos grupos de cohortes: 1934-1943 y 1964-1973 ${ }^{8}$. Los autores concluyen que el efecto de la educación del padre, aunque aún es importante, ha disminuido en el tiempo, principalmente como resultado del incremento en la probabilidad de obtener un grado universitario entre los individuos de padres menos educados ${ }^{9}$. Por su parte, Güell et al. (2007) utiliza un enfoque novedoso para examinar los cambios en la MEI en España. Dado que muchas personas con el mismo apellido pertenecen a la misma familia, existen correlaciones en la educación por apellido. Los autores encuentran que el contenido informativo de los apellidos se ha incrementado a lo largo del tiempo en España, sugiriendo que la persistencia educativa intergeneracional aumentó, a pesar del gran incremento observado en los logros educativos de la población (Black y Deveraux,

\footnotetext{
${ }^{7}$ No obstante, debe señalarse que existen varios estudios previos que analizan los cambios en el efecto de distintas variables del entorno socioeconómico familiar sobre el logro educativo de los hijos (Blanden y Machin, 2004, Machin y Vignoles, 2004, Dustman, 2004, entre otros).

${ }^{8}$ Los autores utilizan una técnica descriptiva de descomposición del cambio en la probabilidad de completar estudios superiores en dos componentes que denominan efecto del entorno paterno y efecto expansión. El primer efecto mide el impacto del crecimiento en la educación de los padres y el segundo, cuantifica los aumentos en la tasa de transición intergeneracional. También estiman un modelo logit multivariado.

9 Además, el análisis de descomposición revela que la tendencia creciente en la participación en la educación superior responde principalmente al efecto mecánico del aumento en los niveles de educación de los padres y no tanto a un incremento en las tasas de movilidad educativa.
} 
2011). En tanto que, Daouli et al. (2010) observan que la MEI aumentó entre las mujeres en Grecia durante los últimos 30 años. ${ }^{10}$ Sin embargo, la educación de las hijas aún depende de la educación de sus padres, especialmente la de su madre. Di Paolo (2012) analizan, en Italia y España, las probabilidades predichas de completar estudios superiores para individuos con padres que tienen distintos niveles educativos y sus cambios a través de las cohortes de nacimiento desde 1940 hasta 1980. Los resultados sugieren que las disparidades en las oportunidades de completar la educación terciaria tendieron a incrementarse a lo largo del tiempo en Italia, pero se redujeron en España. Otro estudio que también examina los cambios temporales de la MEI en Italia, para un grupo de cohortes de nacimiento similares, es el de Checchi et al. (2013). Con este objetivo, implementan una descomposición del CCE estimado para varias cohortes de nacimiento, entre distintos subgrupos definidos según el nivel educativo del padre. Los autores concluyen que en Italia se observa una elevada polarización en las oportunidades educativa que se mantiene en el tiempo, con una persistencia educativa principalmente atribuible, por un lado, a los hijos de padres sin educación $y$, por otro, a hijos cuyos padres completaron estudios superiores. Por último, el artículo de Ranasinghe (2015) analiza los cambios en la transmisión intergeneracional de la educación en Australia para las cohortes de nacimiento de 1942 a 1992. Los resultados sugieren que mientras el nivel general de MEI se incrementó a lo largo del tiempo, existen importantes diferencias regionales y por género.

Entre los pocos estudios que analizan la evolución temporal de la MEI en algún país en desarrollo (no latinoamericano) se encuentran el realizado por Tansel (2011) para Turquía, el de Magejo et al. (2014) para Sudáfrica y el de Azam y Bhatt (2014) para India. Los tres estudios computan como principales indicadores de MEI el CPE así como el CCE. Además tanto Tansel (2011) como Magejo et al. (2014) estiman modelos probit ordenados para el nivel educativo de los hijos. El estudio de Tansel (2011) es el primero que analiza, en Turquía, las tendencias temporales en la correlación entre la educación de padres e hijos a lo largo de distintas cohortes de nacimiento. Los resultados muestran una tendencia creciente en la MEI a partir del CPE pero el patrón temporal es menos claro cuando se utiliza el CCE. En tanto que, Magejo et al. (2014)

\footnotetext{
${ }^{10}$ Con este fin, utilizan la técnica de descomposición propuesta por Hanslin et al. (2007), distintos indicadores de movilidad, análisis de regresión así como modelos convencionales de elección discreta.
} 
examinan las tendencias en la transmisión intergeneracional de la educación entre los sudafricanos de raza negra a partir de datos para las cohortes de nacimiento de 1954 a 1993. Los autores encuentran una disminución en la persistencia educativa intergeneracional a lo largo de las últimas cuatro décadas que es más fuerte en la cola inferior de la distribución de la educación. Por su parte, Azam y Bhatt (2014) implementan la técnica de descomposición del CCE propuesta por Checchi et al. (2013) para examinar la evolución de la MEI en India entre diferentes castas y estados para las cohortes de nacimiento de 1940 a 1985. Los autores encuentran que la disminución en la correlación en la parte inferior de la distribución educativa de los padres es compensada por un incremento en la parte superior de esa distribución.

Las investigaciones sobre MEI en América Latina, así como sobre movilidad intergeneracional en general $^{11}$, enfrentan como principal dificultad la falta $o$ limitaciones de información sobre características socioeconómicas de padres e hijos para individuos adultos. En este contexto se desarrollaron los de estudios Behrman et al. (1999), Dahan y Gaviria (2001) y Andersen, (2001) que proponen índices de movilidad intergeneracional ${ }^{12}$ que pueden ser computados a partir de la información generalmente disponible en las encuestas de hogares de los países de la región. Éstos fueron utilizados en varios estudios sobre MEI, en particular, para Argentina. En el primero de los artículos mencionados, Behrman et al. (1999) propone el índice proporcional de movilidad educativa intergeneracional (PISMI, por sus siglas en inglés) basado en las brechas de escolaridad $^{13}$ de niños que viven con sus padre y en la magnitud con la que las variables del entorno familiar explican esas brechas. Por su parte, el índice de movilidad social (SMI, por sus siglas en inglés) postulado por Andersen (2001) es similar al anterior (pero difiere en su instrumentación) y se define como 1 menos la proporción de la brecha educativa que es explicada por el entorno familiar. Los valores del SMI, obtenido para 18 países de América Latina a partir de encuestas de hogares realizadas hacia fines de los noventa, indican que Chile, Argentina, Uruguay y Perú se encuentran entre los países con los niveles más altos de movilidad social, en tanto que

\footnotetext{
${ }^{11}$ Ferreira et al. (2013) y Torche (2014) realizan una amplia revisión de la literatura empírica sobre movilidad intergeneracional en América Latina.

${ }^{12}$ Los detalles metodológicos sobre la forma de computar cada uno de estos índices pueden consultarse en Conconi et al. (2008).

${ }^{13}$ La brecha de escolaridad es definida como la diferencia entre los años de educación que un adolescente o adulto joven habría completado si hubiera entrado a la escuela en una edad normal y hubiera avanzado un grado cada año, por una parte y los años de educación actual, por otra.
} 
Guatemala y Brasil presentan los niveles más bajos ${ }^{14}$. Por su parte, Dahan y Gaviria (2001) desarrollan un índice de movilidad social basado en la correlación de la escolaridad entre hermanos (SCI, por sus siglas en inglés) que mide el grado con el cual sus resultados educativos pueden ser explicados por el entorno familiar. Los valores obtenidos de este índice para 16 países de Latinoamérica, a partir de encuestas de hogares relevadas hacia fines de los 90s, muestran discrepancias substanciales en la movilidad intergeneracional dentro de la región ${ }^{15}$. Asimismo, encuentran una importante brecha de movilidad entre los países de Latinoamérica y los Estados Unidos. Este hallazgo es confirmado por Behrman et al. (2001) quienes, siguiendo la metodología propuesta Dahan y Gaviria (2001), analizan el efecto del entorno familiar sobre el logro educativo de los jóvenes en 19 países de América Latina, el Caribe y los Estados Unidos durante la década de 1990. Los autores también examinan, en algunos países de la región, la evolución del CPE para distintas cohortes de nacimiento. Los resultados indican que mientras la persistencia educativa intergeneracional disminuyó fuertemente entre los países de baja movilidad (Brasil y Colombia), en los países con una movilidad moderada (México y Perú), su caída fue más leve.

Otros estudios más recientes sobre MEI en Latinoamérica, que incluyen entre los países analizados a Argentina ${ }^{16}$, son los realizados por Conconi et al. (2008), Mediavilla y Calero (2010) y Daude y Robano (2015). En su estudio empírico, Conconi et al. (2008) computan los tres índices de movilidad previamente mencionados, para los países de América Latina a principios de los 90s y de los 2000s: el PISMI de Behrman et al. (1999), el SMI de Andersen (2001) y el SCI de Dahan y Gaviria (2001). Los resultados indican que, durante el período considerado, la movilidad en América Latina se incrementó, aunque no en igual magnitud en todos los países. A su vez, los autores reportan evidencia de una relación negativa entre desigualdad y movilidad. Por su parte, Mediavilla y Calero (2010) calculan y comparan el grado de MEI existente en 6 países de la región utilizando datos de encuestas de hogares para 1998-1999. Los resultados

\footnotetext{
${ }^{14}$ Los resultados también muestran que la movilidad social está positivamente correlacionada con el PBI y el nivel educativo general pero no está relacionada en una forma obvia con la desigualdad.

${ }^{15}$ Los autores también encuentran que la movilidad se incrementa con el ingreso per cápita y la escolaridad media pero está débilmente asociada con los gastos públicos en educación.

${ }^{16}$ Existen también otros estudios para la región que, si bien, no analizan directamente la MEI, evalúan el efecto que tienen distintas variables del entorno familiar (como la educación de los padres) sobre el logro educativo de los hijos. Entre estos, pueden mencionarse los artículos de Castañeda y Aldaz-Carroll (1999) así como Aldaz-Carroll y Morán (2001).
} 
obtenidos de la estimación del CPE, del CCE así como de matrices de transición muestran menores niveles de MEI en estos países en comparación con países más desarrollados. En un artículo reciente, Daude y Robano (2015) examinan los niveles de MEI en 18 países de América Latina a partir de la encuesta de opinión pública Latinobarómetro del 2008 y obtienen valores de persistencia educativa intergeneracional relativamente bajos en Argentina así como en Costa Rica. En el otro extremo, entre los países con los niveles inmovilidad intergeneracional más altos se encuentran Brasil, Ecuador, República Dominicana y Guatemala. Esta persistencia está correlacionada con altos retornos a la educación, relativamente baja progresividad en las inversiones públicas en educación y desigualdad de oportunidades. Con relación a este último hallazgo, debe destacarse que pocos estudios analizan empíricamente, como éste, las relaciones entre un indicador de MEI y un índice que mide la desigualdad de oportunidades ${ }^{17}$. Asimismo, los autores también observan, para el conjunto de países, que mientras el CPE disminuyó entre las cohortes más jóvenes, el $\mathrm{CCE}$ no muestra cambios significativos entre cohortes.

Existen también varias investigaciones sobre MEI para países de América Latina que examinan su evolución a lo largo de distintas cohortes de nacimientos. Entre ellas, deben destacarse las desarrolladas por Torche, (2010) para cuatro países de la región ${ }^{18}$ y por Binder y Woodruff (2002) para México que se proponen analizar el efecto del contexto macroeconómico sobre la evolución de la $\mathrm{MEI}^{19}$. En ambos casos, se encuentra un efecto negativo de las crisis económicas sobre la MEI. Por otra parte, en su artículo para Colombia, Cartagena (2003) analiza, a partir de los datos de la encuesta de calidad

\footnotetext{
${ }^{17}$ Otro estudio que también desarrolla una análisis similar es el de Brunori et al. (2013) para 41 países (entre ellos, 6 son Latinoamericanos). Los autores encuentran que la desigualdad de oportunidades está negativamente correlacionada con la movilidad intergeneracional de la educación y de los ingresos. La desigualdad de oportunidades es evaluada a partir de una índice que mide la desigualdad de una distribución contrafáctica donde los resultados de los individuos son reemplazados por una valuación del conjunto de oportunidades del grupo (o "type") al que pertenece el individuo en función de sus circunstancias de origen.

${ }^{18}$ Este artículo realiza un análisis comparativo de la relación entre las tendencias en la MEI y la crisis económica de la década de 1980 en 4 países de América Latina (Brasil, Chile, México y Venezuela). Los resultados obtenidos muestran un incremento en la desigualdad de oportunidades educativas en el nivel secundario y superior entre las cohortes que experimentaron la crisis económica. En cambio, se aprecia una disminución en la desigualdad de oportunidades en el nivel educativo más bajo que se explica, según la autora, por su universalización.

${ }^{19}$ Los estudios de Marteleto et al. (2012), para cuatro países latinoamericanos (Brasil, Chile, México y Uruguay) y el de Rucci (2003), para Argentina, desarrollan un análisis similar. Aunque no examinan la MEI directamente, los autores investigan los efectos de las crisis económicas sobre las tasas de asistencia escolar de los niños y adolescentes según el nivel educativo del jefe de hogar.
} 
de vida de 1997, que contiene información sobre la educación de los jefes de hogar y sus padres, los cambios en MEI entre las cohortes de nacimiento desde 1929 hasta 1978. Los resultados muestran una disminución en la tasa de crecimiento de la MEI desde mediados de la década de 1960. En tanto que, Núñez y Miranda (2011) examinan los patrones de movilidad intergeneracional del ingreso y de la educación en zonas urbanas de Chile a través de distintas cohortes de nacimiento. Las estimaciones obtenidas sugieren un incremento global en la MEI en las últimas décadas. En un artículo reciente, Gandelman y Robano (2014) observan, a partir de la estimación del SMI (entre otros indicadores), que la MEI disminuyó en Uruguay durante 1982-2010. También encuentran una relación positiva entre la tasa de actividad empresarial y la MI.

Los estudios específicos para Argentina sobre MEI no son tan numerosos. Un primer grupo de investigaciones (Fernández, 2006, Gasparini, 2007 y Navarro, 2010) desarrollan su análisis a partir del índice de movilidad propuesto por Andersen (2001). Además Fernández (2006) estima también el PISMI y el SCI con el objetivo de analizar la existencia y el grado de movilidad social intergeneracional en Argentina con los datos de la Encuesta Permanente de Hogares (EPH) desde 1996 a 2002. Los índices estimados no exhiben cambios dramáticos entre 1996 y 2002. Asimismo, en un estudio que documenta la situación socio-económica en Argentina entre 1992 y 2006, Gasparini (2007) reporta, entre otros indicadores, el SMI. Los valores de este índice obtenidos para adolescentes (13 a 19 años) y adultos jóvenes (20 a 25 años) no revelan mejoras considerables en la movilidad educativa durante el período de análisis. Por su parte, la investigación de Navarro (2010) tiene como objetivo central examinar las diferencias en el grado de movilidad social intergeneracional en Argentina entre adolescentes y jóvenes adultos, utilizando la Encuesta de Educación y Empleo de los Jóvenes (EEEJ) realizada en 2005 en el Gran Buenos Aires $(\mathrm{GBA})^{20}$. Con ese objetivo la autora computa el SMI así como matrices de transición que relacionan el nivel educativo alcanzado por los hijos con el obtenido por sus padres. Los resultados sugieren que la movilidad intergeneracional es bastante menor para los jóvenes adultos en comparación con los adolescentes. Según la autora, estos hallazgos sugieren que al momento de

\footnotetext{
${ }^{20}$ Esta encuesta contiene información retrospectiva sobre la educación de los padres de todos los jóvenes de 15 a 30 años de edad entrevistados.
} 
decidir si continuar estudiando más allá del nivel de escolaridad obligatorio, los jóvenes adultos están fuertemente afectados por el nivel educativo de sus padres.

Por otra parte se encuentran las investigaciones para Argentina que utilizan otras medidas de MEI computadas a partir de bases de datos novedosas con información retrospectiva sobre los padres (FIEL, 2008, Jorrat, 2000, 2010 y Casal y Paz Terán, 2012). Así, la investigación de la Fundación de Investigaciones Económicas Latinoamericanas (FIEL) (2008) se desarrolló en base a una encuesta específica sobre movilidad social (la Encuesta de Movilidad Social) realizada en el 2007 a 1000 hogares del Gran Buenos Aires (GBA). Los resultados obtenidos con relación a MEI muestran una movilidad bastante más baja que la observada en los países más avanzados y menor que la observada en términos del ingreso. También se aprecia que la educación del padre es más importante que la de la madre en la determinación del patrón de persistencia educativa. Asimismo, la MEI muestra una suave forma de "U" a lo largo de las últimas décadas: las generaciones más jóvenes y las mayores exhiben menor movilidad que las intermedias.

Desde una perspectiva sociológica, Jorrat (2000, 2010) y Plotno (2011) también evaluaron el grado de asociación entre orígenes educacionales y logros educativos en Argentina. En el primer estudio, Jorrat (2000) analiza, entre otras cuestiones, la MEI en el GBA a partir de una encuesta específica realizada en 1995 cuyos resultados compara con otro relevamiento de 1961 sobre temas de estratificación y movilidad. Las estimaciones obtenidas muestran que la inmovilidad educativa es importante en el nivel primario y en el universitario tanto en 1961 como en 1995. En un estudio más reciente, Jorrat (2010) utiliza los datos de la Encuesta sobre Estratificación y movilidad social (EEMS) de $2007^{21}$ sobre movilidad social en Argentina y diversos enfoques como regresiones lineales múltiples, regresiones logísticas y matrices de transición. El análisis de la variación de la MEI a través de las cohortes de nacimiento muestra resultados mixtos sobre tendencias crecientes o decrecientes en la MEI. A partir de la misma base de datos (la EEMS) del 2007, Plotno (2011) estima matrices de transición y observa, tanto en la cohorte más joven considerada como en la mayor, un proceso de MEI

\footnotetext{
${ }^{21}$ La encuesta fue realizada por el Centro de Estudios de Opinión Pública de la Universidad Nacional de Buenos Aires (UBA).
} 
mayormente ascendente pero en el que la proporción de hijos con padres sin estudios primarios completos que logra terminar estudios universitarios es extremadamente baja.

Por su parte, Casal y Paz Terán (2012) exploran los cambios que tuvieron lugar en la MEI durante el período 1970-2010, a partir de los datos de los censos nacionales de población realizados en Argentina. Conforme con los valores estimados de los CPE para individuos de 25 a 40 años, concluyen que la persistencia educativa intergeneracional se ha reducido, aunque presenta un aumento entre 1970 y 1980 . No obstante, debe advertirse que las estimaciones reportadas por las autoras podrían estar sesgadas debido a que sólo se utilizan muestras de padres e hijos que habitan juntos puesto que, en los censos, sólo para ellos se dispone de la información necesaria para realizar las estimaciones.

En conclusión, las investigaciones sobre MEI en Argentina son relativamente escasas. Además varios de los estudios existentes estiman índices a partir de encuestas de hogares para adolescentes y jóvenes que residen con sus padres ${ }^{22}$. Asimismo, alguno de estos índices, como los propuestos por Behrman et al. (1999), Andersen (2001) y Dahan y Gaviria (2001), no miden de forma directa la MEI sino que constituyen una aproximación indirecta. Este enfoque indirecto está basado en la medición de la importancia del entorno familiar como determinante de los resultados educativos de niños y jóvenes. Es decir, se asume que cuanto más importante sean las características socioeconómicas de origen como determinantes del acceso a las oportunidades educativas de los jóvenes, mayor será la persistencia de esas características y menor será la movilidad. En cambio, en esta investigación se emplea una base de datos novedosa que contiene información sobre la educación de los hijos y sus padres para todos los individuos adultos entrevistados, independientemente que habiten con sus padres. Esto permite adoptar un enfoque directo para medir y caracterizar la MEI. Por otra parte, ninguno de los estudios mencionados examina formalmente las relaciones entre la MEI observada y el concepto de igualdad de oportunidades en Argentina. Además, como se discutirá a continuación, son muy pocos los estudios, a nivel internacional, que abordan

\footnotetext{
${ }^{22}$ Las excepciones son los estudios de Jorrat $(2010,2014)$ y Plotno (2011) a partir de encuestas específicas con cobertura nacional así como las investigaciones desarrolladas por FIEL (2008) y por Navarro (2010), aunque ambas están limitadas al área del GBA.
} 
de forma explícita esta cuestión ${ }^{23}$. Asimismo, para evaluar los procesos de movilidad intergeneracional observados desde el enfoque de igualdad de oportunidades se utiliza una técnica novedosa recientemente propuesta por Anderson et al. (2014) que será aquí implementada por primera vez para otro país ${ }^{24}$. Esta último constituye una de las principales contribuciones del presente estudio a la literatura existente.

\section{Movilidad intergeneracional e igualdad de oportunidades}

Desde una perspectiva normativa, existe un creciente consenso en privilegiar la igualdad de oportunidades, una característica generalmente deseable para la sociedad, sobre la igualdad de resultados como el objetivo relevante para orientar políticas públicas. Sin embargo, debe señalarse que este orden de prioridades en materia de políticas sociales ha sido actualmente cuestionado, entre otros, por Dubet (2011) quien, luego de analizar estos dos modelos de justicia social, igualdad de resultados o posiciones e igualdad de oportunidades, defiende el primero sobre el segundo, dejando claro que tal elección no significa que deba ignorarse la igualdad de oportunidades sino que establece una prioridad, considerando que la acción pública consiste en jerarquizar los objetivos. La crítica general de Dubet a la concepción de la igualdad de oportunidades radica en su relación con el principio meritocrático de justicia social. Así, para el autor, esta concepción de justicia consiste en ofrecer a todos la posibilidad de ocupar las mejores posiciones en función de un principio mertiocrático, sin cuestionar la brecha que existe entre las posiciones. A favor del modelo de igualdad de resultados o posiciones, el autor sostiene que resulta más favorable a los más débiles porque, de manera indirecta, favorece más la igualdad de oportunidades que ese modelo de justicia e incluso porque la igualdad relativa entre las posiciones sociales es un bien sí mismo, pues las desigualdades terminan afectando a la sociedad en su conjunto. Sin ignorar el planteo de Dubet (2011), reconociendo que esta discusión excede los límites de la

\footnotetext{
${ }^{23}$ El estudio de Daude y Robano (2015) es uno de los pocos que analizan empíricamente las relaciones entre MEI y EOP a partir de indicadores para una y otra dimensión. Pero, a diferencia del análisis aquí desarrollado, no evalúan en qué medida los procesos de MEI observados implican más o menos igualdad de oportunidades.

${ }^{24}$ La técnica propuesta por Anderson et al (2014) es utilizada por los autores para evaluar los progresos en igualdad de oportunidades en Canadá, en Estados Unidos (Anderson y Leo, 2015) y en Alemania (Anderson et al., 2015).
} 
economía y corresponden al campo de la filosofía, se considera que son diversas las razones que justifican el análisis de la relación entre MII e igualdad de oportunidades, más allá del orden de prioridad que le corresponda a este último objetivo en la agenda política.

Las investigaciones que analizan formalmente, desde el punto de vista teórico o empírico, las relaciones entre movilidad intergeneracional e igualdad de oportunidades son relativamente recientes y aún bastante raras. Conforme advierten Van de Gaer et al. (2001), en los estudios empíricos con frecuencia se computan índices de movilidad intergeneracional y luego, implícita o explícitamente y sin un fundamento teórico claro se interpretan los hallazgos a la luz de criterios normativos relacionados con conceptos como el de igualdad de oportunidades. En relación con esto, cabe destacar que la gran mayoría de la literatura empírica que se propone estimar el nivel de MII supone implícitamente la existencia de igualdad de oportunidades cuando las filas de la matriz de transición intergeneracional de ingresos son idénticas o, en general, si la probabilidad que tiene un individuo de alcanzar determinado nivel de ingreso es independiente del ingreso de sus padres. Sin embargo, tal como advierte Roemer (2004) este criterio implica asumir un concepto determinado de EOP que, desde el punto de vista social y normativo, puede ser bastante estricto o "radical" y, por tanto, no totalmente aceptable. El estudio de Roemer (2004), que constituye una de las escasas investigaciones que se propone analizar formalmente la relación entre igualdad de oportunidades y MI, plantea cuatro enfoques para definir la igualdad de oportunidades en función de la inclusión de un conjunto de circunstancias consideradas socialmente no aceptables ${ }^{25}$. Según la visión propuesta por Roemer $(1998,2004)$ de "nivelar el campo de juego", las oportunidades son realmente idénticas cuando todos los individuos que gastaron el mismo nivel de esfuerzo, sin importar su "tipo" 26 , tienen las mismas chances de alcanzar un objetivo. En otras palabas, según este enfoque, existe igualdad de oportunidades cuando los individuos que aplicaron el mismo nivel de esfuerzo tienen las mismas probabilidades de alcanzar un resultado independientemente de sus circunstancias. Por lo tanto el concepto de EOP está íntimamente relacionado con la distinción entre circunstancias y esfuerzo. Las circunstancias son aspectos del entorno que afectan el status

\footnotetext{
${ }^{25}$ Para una descripción detallada de cada uno de los enfoques, ver Roemer (2004)

${ }^{26}$ Para Roemer $(1998,2004)$ un "tipo" es el conjunto de individuos en la sociedad que comparten las mismas circunstancias.
} 
socioeconómico que no se encuentran bajo la responsabilidad del agente. El esfuerzo, en cambio, es el conjunto de acciones que afectan el status de un individuo y por las que se puede responsabilizarlo. En este esquema, Roemer (2004) plantea que la concepción más estricta o "radical" de EOP, denotada por el autor como EOp4, implica que los policy makers deberían nivelar el campo de juego eliminando la influencia no sólo de las conexiones sociales, las inversiones y cultura familiar así como de la transmisión genética de la habilidad sino también las influencias del entorno familiar en la formación de las preferencias y aspiraciones entre los niños ${ }^{27}$. Entonces, el concepto de igualdad de oportunidades implica sólo bajo circunstancias bastantes extremas (las consideradas en el enfoque EOP4), completa MII.

Además, algunos mecanismos de persistencia intergeneracional, como por ejemplo, la herencia genética, existirían incluso en una sociedad en el que las instituciones compensen completamente por las desventajas sociales de origen. Si estos mecanismos determinan fuertemente el éxito socioeconómico, entonces la asociación intergeneracional de resultados podría ser alta aunque existiera igualdad de oportunidades, en un sentido menos estricto (Jenks y Tach, 2006, Torche, 2015 $5^{28}$ ). Por lo tanto, considerar que existe igualdad de oportunidades sólo cuando no hay asociación entre los ingresos de padres e hijos puede ser difícil de aceptar. Por eso, para Roemer (2004) los estudios sobre MI deberían hacer un esfuerzo por testear la existencia de igualdad de oportunidades a partir de visiones menos radicales.

Otro estudio que examina la relación entre igualdad de oportunidades y movilidad, tanto intra como intergeneracional del ingreso, es el de Benabou y Ok (2001). Si se considera el proceso de movilidad como uno potencialmente igualador de oportunidades se sigue que lo que se debería medir es el grado con el cual aquella produce este efecto nivelador. Esto, a su vez, se corresponde, según Benabou y Ok (2001) con una noción de redistribución del ingreso aunque estocástica. Así, al igual que un esquema tributario mapea ingresos pre-impuestos en ingresos post-impuestos, el

\footnotetext{
27 Según Roemer (2004) aunque nuestras preferencias pueden haber sido inculcadas en gran medida durante la niñez, uno adquiere responsabilidad por ellas cuando se identifica con esas preferencias. Entonces, una persona debería ser responsabilizada por sus preferencias siempre y cuando esté de acuerdo con ellas o las asuma voluntariamente como propias.

${ }^{28}$ En este artículo, la autora desarrolla una revisión de los estudios sobre MI, en particular, de aquellos que examinan sus vínculos con la desigualdad económica, los mecanismos de movilidad y la validez de la MI como medida de desigualdad de oportunidades.
} 
proceso de movilidad mapea ingresos iniciales en ingresos futuros esperados $o$, en general, en niveles esperados de bienestar intertemporal. Entonces, el grado con el cual la movilidad de ingresos es más o menos igualadora de oportunidades puede ser medida por el grado de progresividad de ese mapeo, en el sentido de tener una tasa "impositiva" promedio decreciente. A partir de esta idea los autores caracterizan teóricamente los procesos de movilidad en función de su progresividad o potencial de igualación de oportunidades y ofrecen un criterio simple para determinar si un proceso es más progresivo (igualador de oportunidades) que otro. También demuestran cómo este ordenamiento se relaciona con el análisis del bienestar social.

Un estudio que adopta un enfoque teórico relacionado con el propuesto por Benabou y Ok (2001) en el contexto específico de la medición del grado de movilidad intergeneracional es el desarrollado por Van de Gaer et al. (2001) que proponen un "índice de desigualdad de oportunidades" basado en el ingreso esperado de un individuo, condicional a la clase de ingreso de sus padres y a las probabilidades de alcanzar cada una de las clases de ingreso consideradas en la fila relevante de la matriz de transición entre dos generaciones.

Por otra parte, en una investigación reciente Anderson et al. (2014) retoman el análisis de la relación entre MI y el enfoque de EOP advirtiendo las limitaciones de las técnicas existentes para evaluar el progreso de alguna política pública en términos de EOP. Los autores proponen un nuevo enfoque que ellos denominan "qualified equal opportunity" (QEO). Según los autores, las acciones de política que tengan como objetivo la QEO se focalizan en mejorar las oportunidades de vida de los que recibieron una "herencia pobre" de sus padres más que en disminuir las oportunidades de los que recibieron una "herencia rica" 29 . De esta forma surge un programa de QEO con objetivos de movilidad asimétricos para incrementar la movilidad de los pobremente dotados y no hacerlo entre los bien dotados cuando esto implique una pérdida de su bienestar. No obstante, los cambios en el coeficiente del status socioeconómico del padre en una regresión generacional o en los índices de movilidad (como consecuencia de los cambios en las magnitudes relativas de los elementos de la una matriz de

\footnotetext{
${ }^{29}$ En el contexto de la movilidad educativa intergeneracional resulta clara la inviabilidad de una política que incremente las oportunidades educativas de los que provienen de entornos familiares con déficit educativo, disminuyendo las oportunidades de quienes están bien posicionados en esta dimensión.
} 
transición) no reflejan adecuadamente la naturaleza asimétrica de una política que tiende a igualar oportunidades de ese modo. Además, incluso el enfoque de dominancia estocástica propuesto por Lefranc et al. $(2008,2009)$ que permite identificar la falta de EOP no produce una estadística que indique el grado de cambio o progreso que existe hacia la EOP. Por eso, es necesario replantear el enfoque empírico actual para evaluar la EOP y la lógica de un programa que tenga como objetivo la QEO. Un programa como este no debe ser caracterizado como movimientos hacia la independencia de resultados y circunstancias para todos los grupos. Al contrario, un programa QEO implica movimientos limitados que modifican la distribución conjunta de resultados $\mathrm{y}$ circunstancias de forma diferente hacia la independencia para los que provienen de un entorno desaventajado en comparación con quienes poseen circunstancias ventajosas.

En definitiva, a diferencia de la gran mayoría de los estudios empíricos previos sobre movilidad intergeneracional, en general, y los que examinaron sus cambios temporales en particular, en esta investigación se utiliza en forma explícita el enfoque de igualdad de oportunidades para evaluar la evolución de la movilidad intergeneracional. Concretamente, se busca examinar si los cambios observados en el proceso de MEI implicaron un incremento en los niveles de EOP. Asimismo, a partir del enfoque de QEO, se pretende determinar si esos cambios produjeron mejoras o progresos en las oportunidades enfrentadas por aquellos pertenecientes a los grupos socioeconómicos más desaventajados. Para testear esa hipótesis y evaluar los procesos de movilidad intergeneracional observados en cada cohorte desde la visión de igualdad de oportunidades, se implementa el criterio propuesto por Anderson et al. (2014) que se describe en la próxima sección.

\section{Metodología}

La movilidad intergeneracional puede caracterizarse a partir de distintos enfoques. Los estudios existentes, en general, utilizan dos: uno paramétrico que, normalmente consiste en la estimación de un modelo de regresión intergeneracional, y otro no paramétrico, basado en la estimación de matrices de transición. Cada uno de estos métodos permite estudiar distintos aspectos de la movilidad entre generaciones así como testear diferentes hipótesis de interés en relación con el concepto de igualdad de 
oportunidades. Además, los dos enfoques presentan ventajas y desventajas frente al otro. Por lo tanto, en esta investigación se utilizan ambos para caracterizar el nivel así como la evolución de la MEI en Argentina.

\subsection{Enfoque paramétrico: los modelos de regresión intergeneracional}

La movilidad educativa intergeneracional es típicamente evaluada a través de la estimación del siguiente modelo que refleja los supuestos teóricos del modelo de Becker y Tomes $(1979,1986)$ :

$$
S_{h_{i}}=\alpha+\beta S_{p_{i}}+\varepsilon_{i}
$$

donde $S_{h_{i}}$ es la educación del hijo; $S_{p_{i}}$ es la educación de los padres (generalmente, se utiliza la educación del padre y $\varepsilon$ es un error aleatorio idéntica e independientemente distribuido con media cero y homoscedástico. El parámetro de interés es $\beta$, denominado aquí coeficiente de persistencia educativa (CPE) y mide la fracción de la educación que, en promedio, es transmitida entre generaciones. En otras palabras, $\beta$ resume en un solo número el grado de persistencia generacional de la educación. De manera que (1$\beta)$ mediría el nivel de movilidad educativa intergeneracional. Es importante aclarar que el CPE no captura el efecto causal de la educación de los padres en la educación de los hijos sino que constituye una medida resumen del grado de inmovilidad intergeneracional ${ }^{30}$.

Una segunda medida de persistencia intergeneracional es la correlación entre la educación de padres e hijos que, como señala Chechi et al. (2013), puede ser estimada normalizando los años de educación de hijos y padres por sus correspondientes desvíos estándares

\footnotetext{
${ }^{30}$ En general, es inevitable que esta media sobrestime cualquier relación causal que exista, como resultado de la correlación entre la educación de los padres y otras variables explicativas en la ecuación estructural del logro educativo de los hijos. Por esto, se considera que el CPE es un indicador del efecto total de la educación de los padres sobre la educación de los hijos que captura el efecto directo de la educación del padre como su efecto indirecto, a través de su correlación con otros factores relevantes determinantes de la educación de los hijos, como la habilidad o el ingreso paterno. Por esto, no se incluyen en el modelo otras variables de control porque el objetivo es obtener una medida, tanto su efecto directo o causal como su efecto indirecto a través de todos los factores relacionados con la educación que se transmiten entre generaciones.
} 


$$
\frac{S_{h_{i}}}{\sigma_{h}}=\alpha+\rho \frac{S_{p_{i}}}{\sigma_{p}}+\varepsilon_{i}
$$

El parámetro $\rho$ es el coeficiente de correlación educativa intergeneracional (CCE). Esta medida se relaciona con la estimación por Mínimos cuadrados ordinarios (MCO) de $\beta$ en (1) de la siguiente forma:

$$
\hat{\beta}=\frac{\sigma_{h p}}{\sigma_{p}^{2}}=\rho_{h p} \frac{\sigma_{h}}{\sigma_{p}}
$$

donde $\sigma_{h p}$ es la covarianza estimada entre la educación de hijos y padres, $\sigma_{h}$ es el desvío estándar estimado de la educación del hijo y $\sigma_{h}$ es el desvío estándar estimado de la educación del hijo. De esta forma, se aprecia claramente que la diferencia entre $\beta$, el CPE, y $\rho$, el CCE, es que el primero, toma en cuenta también los cambios en la desigualdad educativa en la generación de los hijos y los padres, ofreciendo una medida relativa de movilidad intergeneracional ${ }^{31}$. En cambio, el CCE es una medida de persistencia educativa intergeneracional depurada de la posible evolución de la dispersión de los resultados educativos en cada generación (Chechi et al., 2013). Si la varianza de la educación de los hijos es menor que la de los padres, el CCE será mayor que el CPE y viceversa. Cuando la varianza de la educación de los hijos y la de los padres sea idéntica, el CPE será igual al CCE (Ranasinghe, 2015). Cualquier cambio en las varianzas relativas producirá que el CPE y el CCE evolucionen de forma diferente.

Con el objetivo de examinar las tendencias en la MEI durante el período más extenso posible, el análisis se realiza a partir de comparaciones de cohortes nacimientos. Para evaluar los cambios temporales en el CPE (así como en el CCE), además de estimar las ecuaciones (1) y (2) para distintas cohortes de nacimiento por separado, también se considera el siguiente modelo empírico:

$$
S_{h_{i c t}}=\alpha+\beta S_{p_{i c t}}+\Psi D_{c} S_{p_{i c t}}+\Omega D_{c}+\gamma A_{i c t}+\varepsilon_{i c t}
$$

donde $S_{h_{i c t}}$ es la educación del i-ésimo hijo en el año t, nacido en la cohorte c; $S_{p_{i c t}}$ es la educación del padre, $\mathrm{D}_{\mathrm{c}}$ es el vector de variables dummies para cada cohorte; $\mathrm{A}_{\mathrm{i}}$ es la

\footnotetext{
${ }^{31}$ Como señala Blanden (2013), la correlación intergeneracional es una medida de movilidad posicional (rank mobility) es decir, de la asociación entre la posición del hijo y del padre en la distribución de resultados (educación o ingresos).
} 
edad del i-ésimo hijo, el vector de coeficientes $\Psi$ mide los cambios en el CPE ( $\beta$ ) (o en el CCE cuando se consideran la educación de padres e hijos estandarizadas como en (2)) entre cohortes observadas en distintos años, a partir de la interacción entre la cohorte de nacimiento y la educación paterna. Las diferencias en el CPE (o en el CCE) entre cohortes de nacimiento pueden reflejar no solamente los efectos de las inversiones privadas realizadas en la niñez o adolescencia de los hijos sino también, en parte, una exposición distinta a la provisión de inversiones públicas, especialmente las relacionadas con el capital humano. La ventaja de este modelo frente al (1) y (2) estimados por cohortes de nacimiento radica en la posibilidad de incluir efectos fijos por cohortes y controles por edad, evitando así confundir sus efectos. No obstante, los niveles educativos generalmente exhiben poco o ningún efecto del ciclo vital después de la edad en que la mayoría de los individuos dejan de estudiar y entran al mercado laboral (Núñez y Miranda, 2011).

A partir del modelo de regresión intergeneracional es posible realizar una primera evaluación de los niveles de persistencia educativa entre generaciones desde el enfoque de igualdad de oportunidades. Según Anderson et al. (2014) una mejora en la igualdad de oportunidades entre los grupos socioeconómicos más desaventajados (es decir, en la QOE) hará que la relación entre la educación de padres e hijos se vuelva cada vez más convexa (o menos cóncava) a lo largo de las cohortes sucesivas. Es decir, un aumento en la QOE producirá que la heteroscedasticidad presente una relación negativa con la educación del padre que se vuelva cada vez más negativa a medida que se consideran niveles educativos paternos más altos. Esto implica que la varianza será mayor entre los hijos de padres con niveles educativos más bajos, lo que constituye una medida del grado con el que los estos hijos han sido separados de sus circunstancias de origen $^{32}$. Entonces, el modelo considerado para evaluar esto es el siguiente:

$$
S_{h_{i}}=\beta_{0}+\beta_{1} S_{p_{i}}+\beta_{2} S_{p_{i}}^{2}+\varepsilon_{i}
$$

donde $E\left(\varepsilon_{i}\right)=0$ y $E\left(\ln \varepsilon_{i}^{2}\right)=\alpha_{0}+\alpha_{1} S_{p_{i}}$, es decir, la heteroscedasticidad se modela considerando que el logaritmo del error cuadrático es una función del nivel educativo

\footnotetext{
${ }^{32}$ Ver en Anderson et al. (2014) la derivación formal de este argumento.
} 
del padre. Por lo tanto, se estima una regresión secundaria en base a los residuos a fin de examinar los cambios en la varianza entre las cohortes de nacimiento:

$$
\ln \varepsilon_{i}^{2}=\alpha_{0}+\alpha_{1} S_{p_{i}}+\eta_{i}
$$

La hipótesis de QOE implica que la igualdad de oportunidades para los grupos de hijos más desaventajados (en términos de la baja educación de sus padres) será mayor si el coeficiente $\beta_{2}$ es cada vez menos negativo (es decir, la relación entre la educación de padres e hijos es cada vez menos cóncava) y el coeficiente $\alpha_{1}$ es cada vez más negativo entre las sucesivas cohortes de nacimiento. No obstante, esta forma de evaluar la MEI desde el enfoque de EOP presenta limitaciones porque el modelo de regresión intergeneracional impone restricciones funcionales y promedia los efectos entre los distintos grupos educativos considerados.

Por otra parte, como advierten Chechi et al. (2013), los cambios en el CPE o en el CCE no capturan solamente las modificaciones en la transmisión de la educación entre padres e hijos sino también diferentes fenómenos tales como el incremento secular en la escolaridad de la población y los cambios en la educación obligatoria. Esto se observa con claridad a partir de la siguiente expresión alternativa para el CCE:

$$
\rho_{h p}=\int \underbrace{\left(S_{h}-E\left(S_{h}\right)\right)\left(S_{p}-E\left(S_{p}\right)\right)}_{(a)} \underbrace{\operatorname{Pr}\left(S_{h} \mid S_{p}\right)}_{(b)} \underbrace{\operatorname{Pr}\left(S_{p}\right)}_{(c)} \text { (7) }
$$

De esta forma se aprecia que $\rho_{h p}$ puede cambiar a lo largo del tiempo por:

- $\quad$ cambios en la dispersión de la educación (estandarizada) de los hijos o de los padres alrededor de sus medias respectivas (término (a)),

- cambios en la distribución de la educación de los hijos condicional a las de sus padres (término (b)),

- cambios en la distribución no condicional de la educación de los padres (término (c)).

Los cambios en el término (a) pueden deberse a una convergencia uniforme hacia mayores niveles de educación. En tanto que el término (c) podría cambiar a causa de modificaciones en contexto institucional como consecuencia del desarrollo de un país y de los incrementos en el nivel de educación obligatoria entre los padres a lo largo de las generaciones. En cambio, el término (b) es el que resulta más relevante desde el punto de vista de la persistencia o transmisión educativa intergeneracional. Por esto, y 
por las otras limitaciones ya mencionadas del modelo de regresión intergeneracional, en esta investigación el análisis empírico se encuentra centrado principalmente en la distribución de la educación de los hijos condicional a la educación de sus padres. Esta puede ser caracterizada a partir del enfoque no paramétrico que se describe a continuación.

\subsection{Enfoque no paramétrico: matrices de transición y la técnica del overlap}

Las matrices de transición documentan los movimientos de los individuos entre diferentes clases o estados, específicamente las matrices intergeneracionales de transición por niveles educativos indican la probabilidad que tiene un hijo de alcanzar un determinado nivel educativo, condicional al nivel educativo del padre. Este método permite observar no sólo si existe más o menos movilidad intergeneracional en los distintos tramos de la distribución de la educación sino, también, la dirección de la movilidad (Jenkins y Siedler, 2007). Al mismo tiempo, las matrices de transición permiten analizar las asimetrías y otras no linealidades en la MEI. Sin embargo, una de las desventajas de este método es que no ofrece una medida única de movilidad que facilite las comparaciones temporales o de otro tipo. Y, aunque a partir de las matrices de transición es posible obtener indicadores que resuman las transiciones observadas, tal como advierten Van der Gaer et al. (2001), los índices existentes de MEI no ofrecen una buena medida del grado de igualdad de oportunidades. Sin embargo, el interés por este aspecto está siempre presente, implícita o explícitamente, cuando se analizan matrices de transición intergeneracional.

Formalmente, sea $S_{h}$ la educación del hijo y $S_{p}$ la educación del padre, una matriz de transición intergeneracional describe los movimientos entre $S_{h}$ y $S_{p}$ y constituye una transformación de una función de distribución acumulada dada por $K\left(S_{h}\right.$, $S_{p}$ ) que captura completamente el movimiento entre $S_{h}$ y $S_{p}$. Para construir esta matriz de transición a partir de $K\left(S_{h}, S_{p}\right)$ es necesario determinar el número de clases (o particiones) a considerar. Suponiendo m clases en cada distribución marginal de la educación de padres e hijos y que los límites de estas clases son respectivamente $0<\xi_{1}<\ldots<\xi_{m-1}<\infty$ y $0<\varsigma_{1}<\ldots<\varsigma_{m-1}<\infty$, la matriz de transición resultante es 
denotada por $P=\left\{p_{i j}\right\}$ y cada elemento $p_{i j}$ es la probabilidad condicional de que un hijo se mueva al nivel educativo $\mathrm{j}$ dado el nivel educativo i que tiene su padre:

$$
p_{i j}=\frac{\operatorname{Pr}\left(\varsigma_{i-1} \leq S_{p} \leq \varsigma_{i} \mathrm{y} \xi_{j-1} \leq S_{h} \leq \xi_{j}\right)}{\operatorname{Pr}\left(\varsigma_{i-1} \leq S_{p} \leq \varsigma_{i}\right)}
$$

El denominador $\operatorname{Pr}\left(\varsigma_{i-1} \leq S_{p} \leq \varsigma_{i}\right)=\pi_{i}$ mide la probabilidad de que un individuo se encuentre en la clase i de $S_{p}$. Como señalan Formby et al. (2004) $\pi_{i}$ y $p_{i j}$ pueden ser interpretadas respectivamente como la proporción de individuos en la clase i de $S_{p}$ y la proporción de individuos en la clase i-ésima de $S_{p}$ que se mueve a la clase j de $S_{h}$.

Conforme advierten Formby et al. (2004), la mayoría de los estudios empíricos sobre movilidad, en general así como sobre movilidad intergeneracional, en particular, han descuidado la implementación de algún procedimiento de inferencia estadística a partir de las matrices de transición y de los índices de movilidad que estiman. Sin embargo, es clara la importancia de computar y evaluar los errores estadísticos asociados con las probabilidades de transición. Esto es particularmente relevante en esta investigación considerando el objetivo de examinar la evolución o cambios temporales en la MEI. Entonces, a fin de determinar la existencia de una diferencia estadísticamente significativa de las probabilidades de transición intergeneracional estimadas en distintos períodos se estiman sus respectivos errores estándares utilizando el método bootstrap.

Con el objetivo de evaluar en qué medida los procesos de movilidad educativa intergeneracional observados y caracterizados a partir de las matrices de transición, generaron cambios en los niveles de igualdad de oportunidades educativas existentes, se utiliza la técnica propuesta por Anderson et al. (2014). Ésta consiste básicamente en medir cuán cerca se encuentra la densidad conjunta observada de los resultados (niveles educativos, en este caso) de padres e hijos de la densidad que refleja independencia (EOP) o independencia condicional (QEO) en esos resultados. Para esto se mide el grado de solapamiento (overlap) de las dos distribuciones. Las principales ventajas de esta medida son su fácil implementación, sus propiedades estadísticas (es asintóticamente normal cuando está basada en una muestra aleatoria, permitiendo, en consecuencia, realizar inferencia) y su adaptabilidad para examinar variables continuas y discretas así como combinaciones en múltiples dimensiones. En concreto, la medida 
de overlap (OV) para variables discretas (como las consideradas en este caso) ${ }^{33}$ de la siguiente forma:

$$
O V=\sum_{i \in I} \sum_{k \in K} \min \left\{j_{i, k}^{O}, j_{i, k}^{E}\right\}
$$

donde $\mathrm{j}_{\mathrm{i}, \mathrm{k}}^{\mathrm{O}}$ es el elemento típico de la matriz de densidad conjunta observada $\mathbf{J}^{\mathbf{O}}$ y $\mathrm{j}_{\mathrm{i}, \mathrm{k}}^{\mathrm{E}}$ es el elemento típico de la matriz de densidad conjunta teórica $\mathbf{J}^{\mathbf{E}}$. Sean el vector de probabilidad de resultados del padre $\left[p_{1}, p_{2}, \ldots, p_{k}\right]$ y el del hijo $\left[h_{1}, h_{2}, \ldots, h_{i}\right]$ de manera tal que $p_{k}=\operatorname{Pr}(k)$ para los padres y $h_{i}=\operatorname{Pr}(i)$ para los hijos, entonces el elemento típico $j_{i, k}=\operatorname{Pr}(i, k)$ de una matriz de probabilidades conjuntas $\mathbf{J}$ es la probabilidad de observar a un par padre-hijo (i,j). En este caso, $p_{k}=\sum_{i} j_{i, k}$ y $h_{i}=\sum_{k} j_{i, k}$.

Esta técnica es fácilmente adaptable para examinar no solamente la hipótesis de independencia sino cualquier otra hipótesis de interés. Anderson et al. (2014) describen la medida de overlap gráficamente a partir de la siguiente figura:

\section{Gráfico 1. Medida $O V$ entre las densidad f y g.}

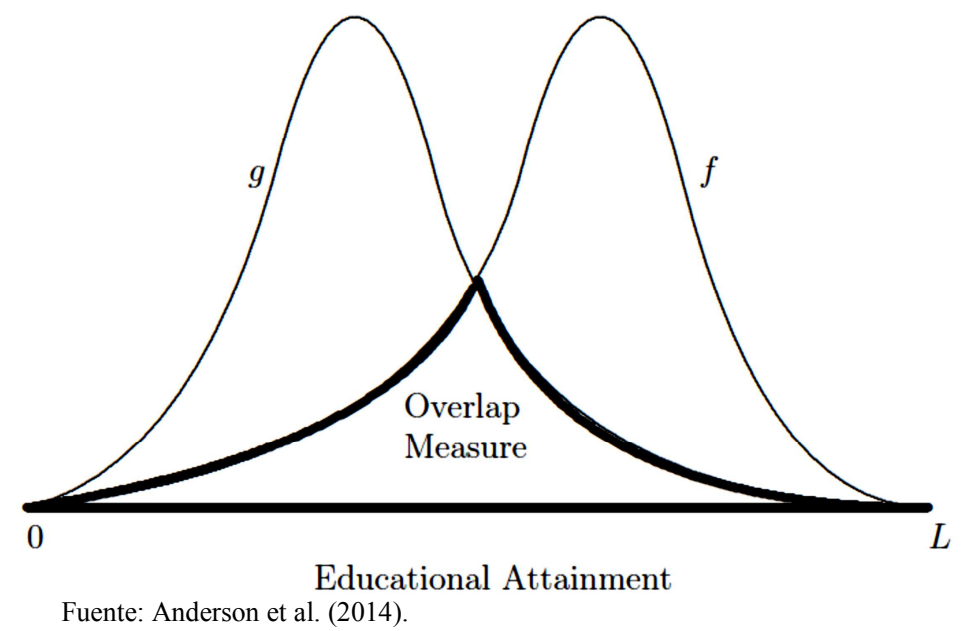

En el Gráfico 1, las distribuciones $\mathrm{f}$ y $\mathrm{g}$ corresponden a las distribuciones observadas y teóricas respectivamente. Se puede notar fácilmente que cuanto más

\footnotetext{
${ }^{33}$ En la implementación empírica se consideran los máximos niveles educativos alcanzados por padres e hijos. Ver la sección 5 para más detalles.
} 
coincidan f y g, $\boldsymbol{O} \boldsymbol{V}$ tenderá en mayor medida a 1 , mientras que cuanto más se diferencien, más se aproximará el índice a 0 .

Para examinar la hipótesis de independencia o EOP, la medida OV adopta la siguiente forma:

$$
O V^{E O P}=\sum_{i \in I} \sum_{k \in K} \min \left\{j_{i, k}^{O}, h_{i} p_{k}\right\}(10)
$$

En tanto que para evaluar la hipótesis QOE a fin de identificar si se observan progresos en un segmento particular de la población, la medida OV a considerar para cada grupo de interés es la siguiente:

$$
O V_{k}^{Q E O}=\sum_{i \in I} \min \left\{\frac{j_{i, k}^{O}}{p_{k}}, h_{i}\right\}
$$

En Anderson et al. (2012) se discute la estimación de estas medidas así como de sus errores estándares. Se utiliza aquí el método de bootstrap para computar los errores estándares de los índices $O V$. Un aspecto interesante de esta técnica es su relación con el concepto de polarización. De hecho, el índice $O V$ puede utilizarse también, en otro contexto, como una medida del grado de polarización entre dos grupos definidos, por ejemplo, por raza, ocupación, género, etc ${ }^{34}$.

Esta técnica tiene varias ventajas sobre otros enfoques existentes para evaluar el grado de igualdad de oportunidades, asociado con un proceso de movilidad intergeneracional dado, como el modelo de regresión intergeneracional en (1), los índices de movilidad derivados de matrices de transición y la reciente evaluación de la ausencia de relaciones de dominancia estocástica entre las distribución de resultados de los hijos de diferentes clases de origen definidas en función del resultado alcanzado por los padres (Lefranc et al., 2008, 2009). Así, los cambios en el coeficiente del resultado del padre en una regresión intergeneracional o los cambios en los índices de movilidad (como resultado de las modificaciones en las magnitudes relativas de los elementos de la diagonal principal o fuera de ella) no reflejan adecuadamente la naturaleza asimétrica del proceso de movilidad ni de las políticas de QOE.

\footnotetext{
${ }^{34}$ Para más detalles sobre este punto ver Anderson et al. (2010).
} 
La principal dificultad con las regresiones intergeneracionales radica en que una covarianza igual a cero no implica independencia entre el resultado del hijo y la circunstancia paterna considerada. Además, la especificación lineal de estos modelos de regresión implica que el efecto marginal de la característica paterna particular que se esté analizando (la educación, por ejemplo) es el mismo a lo largo de toda la distribución de esa variable así como de la variable dependiente. Una forma de reducir estos problemas es utilizando la técnica de regresiones por cuantiles (QR) no paramétricas. No obstante, este enfoque no puede ser implementado en este caso dada la naturaleza categórica de las variables de interés (niveles educativos de padres e hijos). Además, el método QR no ofrece una estadística como el índice OV que resume en un solo número el grado de avance en términos de EOP.

Por su parte, una de las principales dificultades del enfoque que utiliza matrices de transición y sus índices derivados es su implementación cuando la variable de resultado y la variable de interés están medidas en distintas métricas y dimensiones discretas y continuas. Otra dificultad con este enfoque es que está basado en particiones arbitrarias del espacio de resultado y circunstancia en segmentos comunes a fin de estimar la matriz de transición. Un problema adicional es que la proximidad a una situación de independencia es difícil de evaluar en este contexto, por eso, generalmente se utiliza alguna función de los componentes de la matriz (como la traza o el determinante de la matriz). Otro problema es la potencial inconsistencia del test ${ }^{35}$ cuando la diferencia entre dos distribuciones continuas es comparada en puntos discretos del soporte, como sucede en este enfoque. Esto implica, en términos prácticos, que una selección incorrecta de la estructura de partición podría llevar a inferir una magnitud de independencia que en realidad no existe (Anderson y Leo, 2015).

Por otra parte, el enfoque de dominancia estocástica, si bien permite evaluar la existencia o falta de EOP, no ofrece una estadística que indique el grado de cambio o progreso hacia la igualdad de oportunidades, como sí lo hace la técnica de overlap a través del índice $O V$ (Anderson et al., 2014).

\footnotetext{
${ }^{35}$ La inconsistencia del test se refiere a que la probabilidad de rechazar correctamente la hipótesis nula no se aproxima a 1 a medida que tamaño de la muestra crece (Anderson y Leo, 2015).
} 


\section{Datos}

Los datos utilizados en el análisis empírico provienen de una encuesta de opinión pública realizada anualmente en América Latina denominada Latinobarómetro. Entre los países a los que se aplica este estudio se encuentra Argentina. Esta encuesta comenzó a realizarse en el año 1995 en 8 países de Latinoamérica y desde ese año continuó implementándose hasta la actualidad ${ }^{36}$. No obstante, recién a partir del 2003, las muestras de la encuesta para Argentina son representativas de la población nacional.

La principal ventaja de esta encuesta para un estudio de la MEI, en relación con otras encuestas disponibles para Argentina como, por ejemplo, la EPH comúnmente utilizada, es la disponibilidad de información retrospectiva sobre la educación de los padres para todos los individuos encuestados, independientemente de que éstos residan o no, al momento de la encuesta, en la misma vivienda que sus padres ${ }^{37}$. Si bien las encuestas Latinobarómetro contienen una adecuada caracterización socioeconómica de cada individuo, no incluyen mediciones precisas del ingreso familiar (Gaviria, 2006).

A fin de examinar los cambios temporales que tuvieron lugar en la MEI se utilizan los datos de las encuestas correspondientes al período 2003-2013, para el que se dispone, como se indicó previamente, de muestras con representatividad nacional, urbana y rural. Como ya fue mencionado, con el objetivo de examinar las tendencias temporales en la MEI durante el período más extenso, el análisis se realiza a partir de comparaciones entre cohortes de nacimiento. La disponibilidad de datos de varias encuestas cross-section permite contar con un mayor número de observaciones (en total 13.200) para realizar estimaciones con más precisión por cohorte de nacimiento. Asimismo, la posibilidad de seguir a cada cohorte de nacimiento durante varios años y observar sus resultados a distintas edades ${ }^{38}$, limita la confusión de los efectos de cohortes y edad que se produce en este tipo análisis longitudinal. No obstante, como ya se mencionó, los niveles educativos generalmente presentan poco o ningún efecto del

\footnotetext{
${ }^{36}$ Si bien la encuesta tiene una periodicidad anual, no se realizó en 1999 ni en 2012.

37 Esto elimina el problema de sesgo de selección por corresidencia potencialmente existente en las estimaciones de movilidad intergeneracional obtenidas a partir de muestras de padres e hijos que cohabitan en una misma vivienda, como por ejemplo, las provenientes de la EPH.

${ }^{38}$ Esto permite también incluir en los modelos de regresión intergeneracional controles por edad, cohorte y año. Ver sección 4 para más detalles.
} 
ciclo vital después de la edad en que la mayoría de los individuos dejan de estudiar y entran al mercado laboral.

En el análisis empírico se consideran a los individuos de 25 a 64 años de edad. Se incluyen individuos mayores a 25 años porque esta es la edad teórica en la que generalmente se concluye la educación formal. En tanto que no se consideran personas mayores de 64 años para evitar posibles sesgos en las estimaciones al incluir cohortes mayores (Daouli et al., 2010). Además, para los individuos de estas cohortes de nacimiento se dispone de una menor cantidad de observaciones en comparación con el resto. Los individuos que tienen de 25 a 64 años en cada uno de los años del período 2003-2013 pertenecen a las cohortes de nacimiento comprendidas entre 1949 y 1988 . A fin de contar con una mayor precisión en las estimaciones, se agruparon estas cohortes en las siguientes cuatro décadas: 1949-1958, 1959-1968, 1969-1978, 1979-1988.

A pesar del menor tamaño muestral de la encuestas Latinobarómetro debe señalarse que no existen diferencias significativas en los años promedio de educación obtenidos de la muestra y los que resultan de encuestas de hogares nacionales (Daude y Robano, 2015) ${ }^{39}$.

El Cuadro 1 presenta algunas estadísticas descriptivas de la educación de los hijos y de sus padres por cohorte de nacimiento (que constituyen las principales variables de interés) así como la cantidad de observaciones disponibles en cada caso. Allí se observan varias estadísticas descriptivas de los años de educación de hijos y padres. No obstante, debe mencionarse que en la encuesta Latinobarómetro esta variable se encuentra truncada en los niveles educativos superiores. Es decir, para aquellos que tienen estudios terciarios o universitarios incompletos o completos se desconoce sus años de educación. En estos casos, se imputó a los individuos los años de educación más frecuentemente observados para esos niveles educativos en otras encuestas de hogares disponibles para Argentina. Sin embargo, como este problema puede generar un error de medición en las variables, se optó por utilizar, en la mayoría de las estimaciones, el nivel educativo.

\footnotetext{
${ }^{39}$ Siguiendo a los autores, se compararon las estimaciones de los años promedio de educación y de la distribución de la población por máximo nivel educativo, obtenidas en cada año a partir de la encuesta Latinobarómetro para Argentina, con las que surgen de las respectivas EPH y no se observaron diferencias estadísticamente significativas.
} 
Cuadro 1. Estadísticas descriptivas por sexo y cohorte de nacimiento

\begin{tabular}{|c|c|c|c|c|c|c|c|c|c|c|c|c|}
\hline \multirow{2}{*}{ Variables } & \multicolumn{4}{|c|}{ Todos } & \multicolumn{4}{|c|}{ Varones } & \multicolumn{4}{|c|}{ Mujeres } \\
\hline & 1949-58 & 1959-68 & 1969-78 & $1979-88$ & 1949-58 & 1959-68 & 1969-78 & 1979-88 & 1949-58 & 1959-68 & 1969-78 & 1979-88 \\
\hline Años promedio de edad & 54.3 & 43.5 & 33.8 & 27.5 & 54.3 & 43.6 & 33.7 & 27.5 & 54.4 & 43.4 & 33.9 & 27.5 \\
\hline \multicolumn{13}{|l|}{ Educación (años) } \\
\hline Media & 9.3 & 10.5 & 11.0 & 11.6 & 9.2 & 10.5 & 11.0 & 11.5 & 9.4 & 10.5 & 11.1 & 11.8 \\
\hline Desvio & 4.0 & 3.7 & 3.5 & 3.1 & 3.9 & 3.6 & 3.5 & 3.1 & 4.1 & 3.7 & 3.5 & 3.1 \\
\hline 25 percentil & 7.0 & 7.0 & 7.0 & 9.0 & 7.0 & 7.0 & 7.0 & 9.0 & 7.0 & 7.0 & 7.0 & 10.0 \\
\hline Mediana & 9.0 & 11.0 & 12.0 & 12.0 & 8.0 & 11.0 & 12.0 & 12.0 & 9.0 & 11.0 & 12.0 & 12.0 \\
\hline 75 percentil & 12.0 & 13.0 & 14.0 & 14.0 & 12.0 & 13.0 & 14.0 & 14.0 & 12.0 & 13.0 & 14.0 & 14.0 \\
\hline \multicolumn{13}{|l|}{ Educación del padre (años) } \\
\hline Media & 6.3 & 7.2 & 8.2 & 9.3 & 6.2 & 7.3 & 8.4 & 9.6 & 6.4 & 7.1 & 8.0 & 9.0 \\
\hline Desvio & 3.9 & 3.8 & 4.0 & 4.1 & 3.8 & 3.6 & 4.1 & 4.0 & 4.0 & 3.9 & 3.9 & 4.1 \\
\hline 25 percentil & 4.0 & 6.0 & 7.0 & 7.0 & 4.0 & 6.0 & 7.0 & 7.0 & 4.0 & 5.0 & 7.0 & 7.0 \\
\hline Mediana & 7.0 & 7.0 & 7.0 & 7.0 & 7.0 & 7.0 & 7.0 & 8.0 & 7.0 & 7.0 & 7.0 & 7.0 \\
\hline 75 percentil & 7.0 & 7.0 & 12.0 & 12.0 & 7.0 & 8.0 & 12.0 & 12.0 & 7.0 & 7.0 & 12.0 & 12.0 \\
\hline \multicolumn{13}{|l|}{ Nivel e ducativo } \\
\hline Primario incompleto & 0.147 & 0.067 & 0.043 & 0.032 & 0.143 & 0.057 & 0.044 & 0.028 & 0.150 & 0.077 & 0.043 & 0.034 \\
\hline Primario completo & 0.326 & 0.266 & 0.214 & 0.144 & 0.335 & 0.268 & 0.212 & 0.165 & 0.317 & 0.265 & 0.216 & 0.126 \\
\hline Secundario incompleto & 0.158 & 0.192 & 0.193 & 0.178 & 0.184 & 0.203 & 0.209 & 0.176 & 0.134 & 0.182 & 0.178 & 0.180 \\
\hline Secundario completo & 0.175 & 0.199 & 0.224 & 0.262 & 0.161 & 0.201 & 0.222 & 0.273 & 0.187 & 0.198 & 0.226 & 0.252 \\
\hline Superior incompleto & 0.080 & 0.114 & 0.152 & 0.231 & 0.074 & 0.139 & 0.170 & 0.216 & 0.085 & 0.093 & 0.135 & 0.244 \\
\hline Superior completo & 0.115 & 0.161 & 0.174 & 0.154 & 0.102 & 0.133 & 0.143 & 0.142 & 0.127 & 0.185 & 0.202 & 0.164 \\
\hline \multicolumn{13}{|l|}{ Nivel educativo del padre } \\
\hline Primario incompleto & 0.377 & 0.277 & 0.207 & 0.145 & 0.387 & 0.252 & 0.201 & 0.130 & 0.368 & 0.299 & 0.213 & 0.158 \\
\hline Primario completo & 0.452 & 0.484 & 0.437 & 0.374 & 0.454 & 0.496 & 0.413 & 0.367 & 0.451 & 0.474 & 0.460 & 0.379 \\
\hline Secundario incompleto & 0.026 & 0.042 & 0.060 & 0.083 & 0.023 & 0.045 & 0.067 & 0.071 & 0.028 & 0.040 & 0.053 & 0.093 \\
\hline Secundario completo & 0.091 & 0.126 & 0.191 & 0.240 & 0.092 & 0.149 & 0.205 & 0.262 & 0.090 & 0.106 & 0.179 & 0.221 \\
\hline Superior incompleto & 0.011 & 0.021 & 0.021 & 0.027 & 0.011 & 0.020 & 0.021 & 0.021 & 0.010 & 0.022 & 0.021 & 0.032 \\
\hline Superior completo & 0.043 & 0.049 & 0.083 & 0.132 & 0.033 & 0.038 & 0.094 & 0.148 & 0.053 & 0.059 & 0.074 & 0.118 \\
\hline $\mathrm{N}^{\circ}$ de observaciones & 1818 & 2027 & 2270 & 1288 & 861 & 948 & 1086 & 611 & 957 & 1079 & 1184 & 677 \\
\hline
\end{tabular}

Fuente: Elaboración propia sobre la base de Latinobarómetro 2003-2013.

Conforme con las estadísticas del Cuadro 1, los años promedios de educación de los hijos y de los padres muestran una tendencia creciente a lo largo de las distintas cohortes de nacimiento, lo que da cuenta del aumento en los niveles de escolaridad de la población. Esto se evidencia también al comparar el máximo nivel educativo alcanzado por los hijos con aquel que presentan sus padres. Así, el porcentaje de la población con sólo estudios primarios (incompletos y completos) es significativamente menor en la generación de los hijos (31\%, en promedio) que en la de los padres (69\%, en promedio). Se puede observar también que los hijos completaron estudios superiores en mayor proporción que sus padres. En promedio, el 15\% de los hijos de 25 a 64 años alcanzaron ese nivel, frente al 7.7\% de los padres. Esta expansión educativa fue particularmente fuerte entre las mujeres de las generaciones más jóvenes. La proporción de hijas con estudios superiores completos supera la proporción de varones con el mismo nivel educativo, particularmente en las cohortes de los 60 s y los 70s. Estos cambios van de la mano con el fuerte crecimiento de la participación femenina en la fuerza laboral durante la última mitad del siglo (Gasparini y Marchionni, 2015). 


\section{Resultados}

\subsection{El modelo de regresión intergeneracional y los cambios en la correlación educativa entre generaciones}

A partir de la estimación del modelo de regresión intergeneracional es posible computar dos medidas de persistencia educativa intergeneracional ampliamente utilizadas en la literatura empírica sobre MEI: el CPE y el CCE. La primera se obtiene de la estimación del modelo (1) que relaciona la educación de los hijos con la de su padre y mide, como ya fue señalado, el grado promedio de persistencia del logro educativo entre generaciones. En tanto que, la correlación intergeneracional (CCE) ajusta la medida anterior por la variación relativa en la educación de padres e hijos, de manera que constituye una medida estandarizada de persistencia educativa elimina la dispersión cross-section que se observa en la educación de cada generación (Checchi et al., 2013, Ranasinghe, 2015). En el Cuadro A1 del Anexo se reportan el CPE y el CCE que surgen de la estimación de las ecuaciones (1) y (2) respectivamente para cada cohorte de nacimiento por separado. En la primera especificación se consideran los años de educación de hijos y padres y en la segunda, su máximo nivel educativo. La tercera especificación corresponde al modelo presentado en la ecuación (4) que se estima en forma conjunta para todas las cohortes de nacimiento incluyendo como variables de control la edad de los hijos, dummies por cohortes de nacimiento y un término de interacción entre la educación de los padres y las dummies de cohorte.

El Gráfico 2 muestra el CPE así como el CCE estimados a partir de los años de educación de padres e hijos, para cada grupo de cohorte de nacimiento ${ }^{40}$. La evolución del CPE es distinta de la que presenta el CCE, particularmente entre los varones. En efecto, el CPE muestra una marcada tendencia decreciente a lo largo de los distintos grupos de cohortes considerados, tanto entre los varones como en el caso de las mujeres. Esta medida disminuyó desde 0.55, en la cohorte más vieja (1949-1958) hasta 0.37, en la cohorte más joven (1979-1988). Esto implica una caída del 33\% en los niveles promedios de inmovilidad educativa intergeneracional medidos por este indicador. En 40 En la columna "Modelo 1" del Cuadro A2 del Anexo se reportan estos coeficientes, sus
correspondientes errores estándares e intervalo de confianza al $95 \%$. 
cambio, el CCE muestra una tendencia decreciente en el total de hijos y entre las mujeres pero no así en el caso de los varones.

\section{Gráfico 2. Coeficiente de persistencia educativa (CPE) y coeficiente de correlación educativa (CCE) por cohortes de nacimiento.}
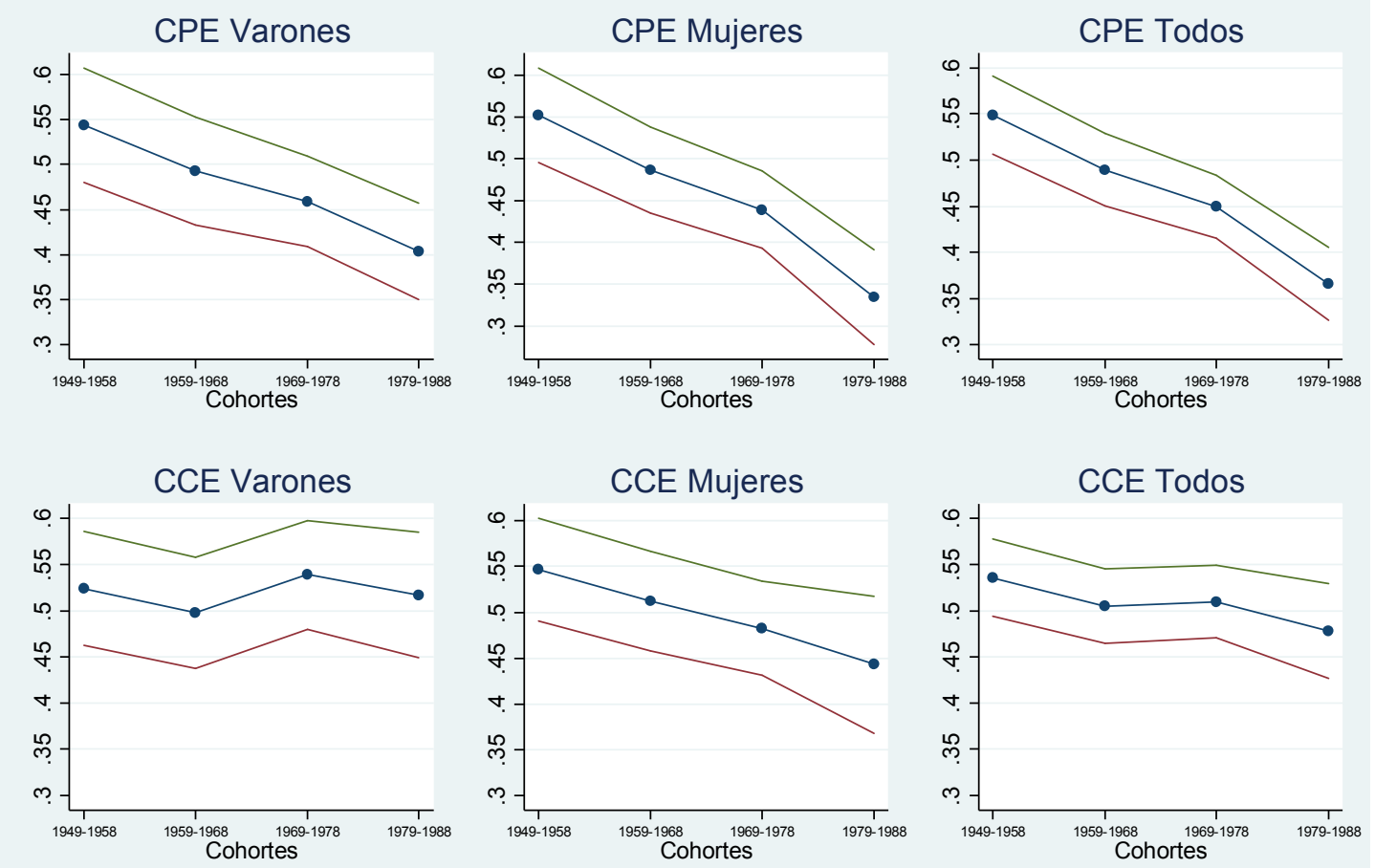

Coeficiente

LI IC 95\%

LS IC $95 \%$

Fuente: Elaboración propia sobre la base de Latinobarómetro 2003-2013.

Además, las cambios entre los grupos de cohortes en la correlación educativa intergeneracional son considerablemente menores que los observados en el CPE. Así, en el caso de las mujeres, mientras la CCE disminuyó un 19\% (desde 0.54 hasta 0.47 ) entre la cohorte más vieja y la más joven, el CPE lo hizo en un 39\% (desde 0.55 hasta 0.33 ). En el caso de los varones, el CCE que asciende, en promedio, a 0.51, no muestra ninguna variación significativa entre las distintas cohortes de nacimiento consideradas. En cambio, su CPE cayó desde 0.54 hasta 0.40 , esto es, un $26 \%$. Con excepción de la cohorte más vieja, en las restantes, el CCE supera el CPE. De acuerdo con la ecuación (2), esto sucede cuando la dispersión de la educación de los hijos es menor que la dispersión de la educación de los padres. Justamente, como puede observarse en el Cuadro 1, el desvío estándar de la educación en la generación de los hijos muestra justamente una tendencia decreciente que no se aprecia entre los padres. Es decir, la 
desigualdad cross-section de la educación entre los hijos se redujo en comparación con la de los padres. Por lo tanto, estos resultados indican que cuando se considera una medida de persistencia educativa que no esté afectada por los cambios en la desigualdad educativa entre generaciones, como es el caso del CCE, los niveles de MEI en Argentina no muestran mejoras significativas a lo largo del período de 30 años que separa a la cohorte más vieja considerada de la más joven.

Asimismo, no se observan diferencias importantes en los niveles promedio de persistencia educativa intergeneracional entre varones y mujeres de la mayoría de las cohortes de nacimiento analizadas. No obstante, en la cohorte más joven (1979-1988) tanto el CPE como el CCE son menores entre las mujeres en comparación con los varones, en aproximadamente un $17 \%$ y $14 \%$. Esto responde a la diferente evolución que muestran los indicadores en ambos grupos, en particular, a la fuerte caída en el CPE y el CCE entre las mujeres de las cohortes más jóvenes que no se observa en el caso de los varones.

Las tendencias temporales no cambian cuando se consideran las otras especificaciones del modelo intergeneracional. En particular, cuando se consideran los años de educación de padres e hijos, no se observan diferencias significativas entre los CPE y CCE computados a partir de la ecuación (4) y aquellos que surgen de la ecuación (1).

A partir del modelo de regresión intergeneracional es posible realizar una evaluación preliminar de los niveles de MEI desde el enfoque de EOP. Con este fin, siguiendo a Anderson et al. (2014) se incluye un término cuadrático de la educación del padre en el modelo $(1)^{41}$ y se analiza el comportamiento de la heteroscedasticidad del modelo. Esto permite contar con una primera aproximación de la medida con la que el proceso de movilidad intergeneracional produjo una mejora en la igualdad de oportunidades, particularmente entre los hijos que pertenecen al grupo socioeconómico más desaventajado, definido en este caso en términos de la educación de sus padres. Cuando esto ocurre, es decir, cuando la denominada QOE es más efectiva, la relación

\footnotetext{
${ }^{41}$ A partir del modelo de Becker y Tomes (1986) se deduce que la relación entre los resultados socioeconómicos de padres e hijos (la educación, en este caso) puede ser cóncava debido a las asimetrías que existen en las restricciones crediticias. Según este modelo, los hijos de padres con baja educación (y bajos ingresos) quienes, al enfrentar restricciones crediticias, no pueden endeudarse para financiar su educación, tienen niveles educativos (e ingresos laborales) menores que otros sin restricciones para financiar sus inversiones en capital humano.
} 
entre la educación de padres e hijos se vuelve menos cóncava a lo largo de cohortes sucesivas. Esto puede ser evaluado a partir del coeficiente $\beta_{2}$ del término cuadrático de la educación del padre en la ecuación (5). Asimismo, cuando la QOE mejora en el tiempo, la varianza de los errores debería ser mayor entre los hijos de padres con niveles educativos más bajos, indicando que estos hijos han logrado separarse más de sus circunstancias de origen. Esto implica que el coeficiente $\alpha_{1}$ de la educación del padre en la regresión secundaria (6) que modela la heteroscedasticidad, debería ser más negativo en las sucesivas cohortes de nacimiento consideradas.

Los resultados de la estimación de la ecuación (5) y (6) se muestran en el Cuadro $2^{42}$. En línea con los CPE previamente reportados, se observa un efecto positivo y significativo de la educación del padre que disminuye a lo largo de las cohortes de nacimiento. Si la proximidad a cero del coeficiente indica cercanía a una situación de independencia entre la educación de padre e hijos, entonces estos resultados sugieren una ganancia en términos de movilidad y de EOP entre las generaciones más jóvenes. Asimismo, en todos los casos se observa que la relación entre la educación de padres e hijos resulta cóncava, conforme surge del signo negativo del coeficiente del término cuadrático. No obstante, esta concavidad disminuye a lo largo del tiempo entre las mujeres, no así en el caso de los varones.

En el modelo secundario que tiene como variable dependiente el logaritmo de los errores al cuadrado, los coeficientes negativos en todas las regresiones indican que la varianza es decreciente con la educación de los padres. Esto sugiere, un mayor nivel de EOP entre los hijos de los grupos socioeconómicos más desaventajados en términos del bajo nivel educativo del padre. También se aprecia, para el total de hijos, un crecimiento, en valor absoluto, en el coeficiente negativo que indicaría una mejora en el nivel de EOP entre los hijos de padres con bajos niveles educativos. Este aumento resulta estadísticamente significativo tanto entre los varones como entre las mujeres,

\footnotetext{
${ }^{42}$ Las estimaciones se realizaron considerando los niveles educativos de padres e hijos dado que los años de educación podrían presentar errores de medición en las encuestas Latinobarómetro (ver la sección 5 sobre los datos, para más detalles). Además, esto permitirá comparar los resultados obtenidos a partir de los modelos de regresión con los derivados de la implementación de la técnica de overlap. Por tanto, el modelo se estima con variables enteras discretas que, como señala Anderson et al. (2014), no permiten implementar un enfoque de regresión por cuantiles y requerirían considerar algún modelo multinomial para su análisis porque los errores de las regresiones son heteroscedásticos. Sin embargo, justamente el comportamiento de esta heteroscedasticidad es lo que se pretende analizar.
} 
cuando se comparan la cohorte más vieja y la más joven, conforme con el test de las diferencias reportadas en el Cuadro 3. Asimismo, se aprecia una reducción en el coeficiente $\beta_{2}$, es decir, en el grado de concavidad de la relación entre la educación de padres e hijos. Esta caída es significativa para todos los hijos así como entre las mujeres cuando se comparan la primera cohorte y la más joven.

\section{Cuadro 2. Regresiones de movilidad educativa intergeneracional y análisis de heteroscedasticidad por cohorte de nacimiento}

\begin{tabular}{|c|c|c|c|c|}
\hline \multirow[b]{2}{*}{ Variables } & \multicolumn{4}{|c|}{ Cohortes } \\
\hline & $\begin{array}{c}1949-1958 \\
{[1]}\end{array}$ & $\begin{array}{c}1959-1968 \\
{[2]}\end{array}$ & $\begin{array}{c}1969-1978 \\
{[3]}\end{array}$ & $\begin{array}{c}1979-1988 \\
{[4]}\end{array}$ \\
\hline \multicolumn{5}{|l|}{ Varones } \\
\hline \multicolumn{5}{|c|}{ Variable dep.: Educación del hijo } \\
\hline Educación del padre & $\begin{array}{c}0.881^{* * * *} \\
(0.154)\end{array}$ & $\begin{array}{c}1.035^{* * *} \\
(0.146)\end{array}$ & $\begin{array}{c}0.846 * * * \\
(0.122)\end{array}$ & $\begin{array}{c}0.582 * * * \\
(0.155)\end{array}$ \\
\hline Educación del padre $^{2}$ & $\begin{array}{l}-0.041 * \\
(0.023)\end{array}$ & $\begin{array}{c}-0.074 * * * \\
(0.022)\end{array}$ & $\begin{array}{c}-0.053^{* * *} \\
(0.017)\end{array}$ & $\begin{array}{l}-0.022 \\
(0.021)\end{array}$ \\
\hline \multicolumn{5}{|l|}{ Variable dep.: $\ln \left(\varepsilon^{2}\right)$} \\
\hline Educación del padre & $\begin{array}{c}0.036 \\
(0.044) \\
\end{array}$ & $\begin{array}{c}-0.193 * * * \\
(0.054)\end{array}$ & $\begin{array}{c}-0.227^{* * *} \\
(0.047)\end{array}$ & $\begin{array}{c}-0.166^{* * *} \\
(0.060)\end{array}$ \\
\hline \multicolumn{5}{|l|}{ Mujeres } \\
\hline \multicolumn{5}{|c|}{ Variable dep.: Educación del hijo } \\
\hline Educación del padre & $\begin{array}{c}1.400 * * * \\
(0.152)\end{array}$ & $\begin{array}{c}1.172 * * * \\
(0.142)\end{array}$ & $\begin{array}{c}0.904 * * * \\
(0.127)\end{array}$ & $\begin{array}{c}0.775 * * * \\
(0.164)\end{array}$ \\
\hline Educación del padre $^{2}$ & $\begin{array}{c}-0.123 * * * \\
(0.023)\end{array}$ & $\begin{array}{c}-0.096^{* * *} \\
(0.022)\end{array}$ & $\begin{array}{c}-0.064 * * * \\
(0.018)\end{array}$ & $\begin{array}{c}-0.059 * * * \\
(0.022)\end{array}$ \\
\hline \multicolumn{5}{|l|}{ Variable dep.: $\ln \left(\varepsilon^{2}\right)$} \\
\hline Educación del padre & $\begin{array}{l}-0.115 \\
(0.075)\end{array}$ & $\begin{array}{c}-0.098^{* *} \\
(0.045)\end{array}$ & $\begin{array}{l}-0.076 \\
(0.052)\end{array}$ & $\begin{array}{c}-0.322 * * * \\
(0.070)\end{array}$ \\
\hline \multicolumn{5}{|l|}{ Todos } \\
\hline \multicolumn{5}{|c|}{ Variable dep.: Educación del hijo } \\
\hline Educación del padre & $\begin{array}{c}1.165^{* * * *} \\
(0.109)\end{array}$ & $\begin{array}{c}1.112 * * * \\
(0.102)\end{array}$ & $\begin{array}{c}0.874 * * * \\
(0.088)\end{array}$ & $\begin{array}{c}0.676^{* * *} \\
(0.114)\end{array}$ \\
\hline Educación del padre $^{2}$ & $\begin{array}{c}-0.087 * * * \\
(0.017)\end{array}$ & $\begin{array}{c}-0.087 * * * \\
(0.016)\end{array}$ & $\begin{array}{c}-0.059 * * * \\
(0.012)\end{array}$ & $\begin{array}{c}-0.040 * * * \\
(0.015)\end{array}$ \\
\hline \multicolumn{5}{|l|}{ Variable dep.: $\ln \left(\varepsilon^{2}\right)$} \\
\hline Educación del padre & $\begin{array}{l}-0.030 \\
(0.040)\end{array}$ & $\begin{array}{c}-0.140 * * * \\
(0.035)\end{array}$ & $\begin{array}{c}-0.197 * * * \\
(0.040)\end{array}$ & $\begin{array}{c}-0.213 * * * \\
(0.043)\end{array}$ \\
\hline
\end{tabular}

Los resultados previos deben ser interpretados con precaución debido a las limitaciones que presentan los modelos de regresión intergeneracional en comparación con un enfoque que permita examinar el comportamiento de toda la función de densidad 
conjunta o condicional. En general, los modelos de regresión imponen restricciones funcionales que no presentan los enfoques no paramétricos como los que se utilizan a continuación.

Cuadro 3. Diferencias del coeficiente que mide el grado de concavidad $\left(\beta_{2}\right)$ y del coeficiente que mide el grado de negatividad de la heteroscedasticidad $\left(\alpha_{1}\right)$ entre cohortes.

\begin{tabular}{|c|c|c|c|c|c|c|}
\hline \multirow{2}{*}{$\begin{array}{l}\text { Dife rencias } \\
\text { entre cohortes }\end{array}$} & \multicolumn{3}{|c|}{ Dife rencias en el coeficiente $\beta_{2}$} & \multicolumn{3}{|c|}{ Diferencias en el coeficiente $\alpha_{1}$} \\
\hline & Varones & Mujeres & Todos & Varones & Mujeres & Todos \\
\hline \multirow[t]{2}{*}[2]{$-[1]$} & -0.033 & 0.027 & 0.002 & $-0.229 * * *$ & 0.017 & $-0.114 * *$ \\
\hline & $(0.310)$ & $(0.402)$ & $(0.929)$ & $(0.001)$ & $(0.842)$ & $(0.032)$ \\
\hline \multirow[t]{2}{*}[3]{$-[2]$} & 0.021 & $0.058^{* *}$ & 0.026 & -0.034 & 0.022 & -0.057 \\
\hline & $(0.461)$ & $(0.045)$ & $(0.196)$ & $(0.638)$ & $(0.751)$ & $(0.280)$ \\
\hline \multirow[t]{2}{*}[4]{$-[3]$} & 0.031 & 0.005 & 0.019 & 0.061 & $-0.247 * * *$ & -0.015 \\
\hline & $(0.246)$ & $(0.859)$ & $(0.345)$ & $(0.421)$ & $(0.005)$ & $(0.796)$ \\
\hline \multirow[t]{2}{*}[4]{$-[1]$} & 0.019 & $0.063^{* *}$ & $0.046 * *$ & $-0.202 * * *$ & $-0.207 * *$ & $-0.186^{* * *}$ \\
\hline & $(0.536)$ & $(0.046)$ & $(0.039)$ & $(0.007)$ & $(0.043)$ & $(0.002)$ \\
\hline \multirow[t]{2}{*}[4]{$-[2]$} & $0.052^{*}$ & 0.037 & $0.044^{* *}$ & 0.028 & $-0.225^{* * *}$ & -0.072 \\
\hline & (0.089) & $(0.232)$ & $(0.043)$ & $(0.734)$ & $(0.007)$ & $(0.191)$ \\
\hline \multirow[t]{2}{*}[3]{$-[1]$} & -0.012 & $0.058 * *$ & 0.028 & $-0.263 * * *$ & 0.039 & $-0.171 * * *$ \\
\hline & $(0.678)$ & $(0.045)$ & $(0.179)$ & $(0.000)$ & $(0.668)$ & $(0.003)$ \\
\hline
\end{tabular}

\subsection{Comparaciones temporales de los patrones de movilidad intergeneracional a partir de matrices de transición}

Otra forma de caracterizar el proceso de movilidad intergeneracional es a partir de matrices de transición. En el Cuadro 4 se reportan las matrices de transición que vinculan el nivel educativo de los hijos con el nivel educativo de sus padres para cada uno de los grupos de cohortes de nacimiento consideradas. En los Cuadros A2 y A3 del Anexo se muestran las matrices de transición estimadas por separado para los varones y para las mujeres.

En general, todas las matrices presentan un patrón relativamente similar. La comparación de los elementos de la diagonal principal de cada una de las matrices de transición revela una clara asimetría en la inmovilidad intergeneracional, con una mayor persistencia de la educación entre los padres y los hijos que se encuentran en el nivel educativo más alto. Así, en promedio, el $47 \%$ de los hijos cuyos padres tienen estudios 
superiores también completaron ese nivel. No obstante, la tasa de persistencia en el nivel superior completo disminuyó entre la cohorte más vieja y la más joven, desde $52 \%$ hasta $37 \%$. Esta caída fue más pronunciada en el caso de las mujeres, entre quienes esta tasa de permanencia disminuyó 23 puntos porcentuales (desde 53\% hasta 30\%). Este cambio, que se aprecia tanto entre los varones como entre las mujeres, implica que en las generaciones más jóvenes, la mayoría de los hijos cuyos padres completaron el nivel superior no logran el mismo nivel educativo, sino uno menor ${ }^{43}$. Además, los cambios mencionados resultan estadísticamente significativos a un nivel de significancia del 5\%.

\section{Cuadro 4. Matrices de transición educativa por cohorte de nacimiento. Varones y mujeres.}

\begin{tabular}{|c|c|c|c|c|c|c|}
\hline \multirow[b]{2}{*}{ Educación padre } & \multicolumn{6}{|c|}{ Educación del hijo } \\
\hline & $\begin{array}{c}\text { Primario } \\
\text { incompleto }\end{array}$ & $\begin{array}{l}\text { Primario } \\
\text { completo }\end{array}$ & $\begin{array}{l}\text { Secundario } \\
\text { incompleto }\end{array}$ & $\begin{array}{c}\text { Secundario } \\
\text { completo }\end{array}$ & $\begin{array}{c}\text { Superior } \\
\text { incompleto }\end{array}$ & $\begin{array}{l}\text { Superior } \\
\text { completo }\end{array}$ \\
\hline \multicolumn{7}{|l|}{ Cohorte 1949-1958 } \\
\hline Primario incompleto & $0.278(0.019)$ & $0.407 \quad(0.021)$ & $0.138(0.014)$ & $0.094(0.011)$ & $0.044(0.009)$ & $0.038(0.008)$ \\
\hline Primario completo & $0.053(0.009)$ & $0.330 \quad(0.018)$ & $0.206(0.015)$ & $0.224(0.014)$ & $0.083(0.011)$ & $0.105(0.012)$ \\
\hline Secundario incompleto & $0.028(0.028)$ & $0.187 \quad(0.064)$ & $0.160(0.061)$ & $0.267(0.065)$ & $0.135(0.056)$ & $0.223(0.069)$ \\
\hline Secundario completo & $0.031(0.015)$ & $0.077 \quad(0.022)$ & $0.083(0.023)$ & $0.304(0.036)$ & $0.210(0.036)$ & $0.296(0.039)$ \\
\hline Superior incompleto & $0.000(0.000)$ & $0.124 \quad(0.082)$ & $0.055(0.054)$ & $0.053(0.051)$ & $0.188(0.099)$ & $0.580(0.122)$ \\
\hline Superior completo & $0.018(0.017)$ & $0.013 \quad(0.013)$ & $0.041(0.024)$ & $0.163(0.041)$ & $0.246(0.055)$ & $0.519(0.062)$ \\
\hline \multicolumn{7}{|l|}{ Cohorte 1959-1968 } \\
\hline Primario incompleto & $0.160(0.017)$ & $0.415 \quad(0.022)$ & $0.182(0.017)$ & $0.130(0.014)$ & $0.054(0.011)$ & $0.059(0.011)$ \\
\hline Primario completo & $0.030(0.006)$ & $0.285 \quad(0.016)$ & $0.231(0.014)$ & $0.238(0.013)$ & $0.093(0.011)$ & $0.123(0.012)$ \\
\hline Secundario incompleto & $0.015(0.015)$ & $0.043 \quad(0.024)$ & $0.260(0.052)$ & $0.299(0.049)$ & $0.221(0.051)$ & $0.162(0.045)$ \\
\hline Secundario completo & $0.005(0.005)$ & $0.042 \quad(0.014)$ & $0.106(0.020)$ & $0.244(0.026)$ & $0.223(0.029)$ & $0.379(0.033)$ \\
\hline Superior incompleto & $0.013(0.013)$ & $0.048 \quad(0.034)$ & $0.044(0.030)$ & $0.121(0.045)$ & $0.413(0.083)$ & $0.360(0.080)$ \\
\hline Superior completo & $0.019(0.019)$ & $0.026 \quad(0.018)$ & $0.062(0.025)$ & $0.148(0.034)$ & $0.220(0.046)$ & $0.525(0.055)$ \\
\hline \multicolumn{7}{|l|}{ Cohorte 1969-1978 } \\
\hline Primario incompleto & $0.109(0.016)$ & $0.401 \quad(0.025)$ & $0.205(0.020)$ & $0.158(0.016)$ & $0.071(0.013)$ & $0.055(0.012)$ \\
\hline Primario completo & $0.036(0.006)$ & $0.249 \quad(0.015)$ & $0.234(0.014)$ & $0.252(0.013)$ & $0.113(0.012)$ & $0.116(0.011)$ \\
\hline Secundario incompleto & $0.030(0.018)$ & $0.111 \quad(0.029)$ & $0.195(0.037)$ & $0.274(0.038)$ & $0.208(0.039)$ & $0.181(0.036)$ \\
\hline Secundario completo & $0.006(0.004)$ & $0.049 \quad(0.012)$ & $0.140(0.018)$ & $0.248(0.020)$ & $0.233(0.023)$ & $0.325(0.025)$ \\
\hline Superior incompleto & $0.000(0.000)$ & $0.055 \quad(0.038)$ & $0.202(0.065)$ & $0.176(0.051)$ & $0.347(0.077)$ & $0.220(0.068)$ \\
\hline Superior completo & $0.006(0.004)$ & $0.007 \quad(0.007)$ & $0.049(0.016)$ & $0.175(0.026)$ & $0.298(0.037)$ & $0.465(0.039)$ \\
\hline \multicolumn{7}{|l|}{ Cohorte 1979-1988 } \\
\hline Primario incompleto & $0.113(0.024)$ & $0.279 \quad(0.036)$ & $0.228(0.031)$ & $0.212(0.030)$ & $0.113(0.026)$ & $0.055(0.019)$ \\
\hline Primario completo & $0.022(0.007)$ & $0.196 \quad(0.020)$ & $0.262(0.020)$ & $0.271(0.020)$ & $0.167(0.019)$ & $0.082(0.014)$ \\
\hline Secundario incompleto & $0.013(0.012)$ & $0.090 \quad(0.030)$ & $0.170(0.036)$ & $0.290(0.043)$ & $0.338(0.050)$ & $0.100(0.032)$ \\
\hline Secundario completo & $0.008(0.006)$ & $0.063 \quad(0.015)$ & $0.099(0.017)$ & $0.319(0.026)$ & $0.276(0.028)$ & $0.235(0.026)$ \\
\hline Superior incompleto & $0.017(0.017)$ & $0.039 \quad(0.038)$ & $0.092(0.053)$ & $0.136(0.058)$ & $0.457(0.093)$ & $0.259(0.083)$ \\
\hline Superior completo & $0.000(0.000)$ & $0.014 \quad(0.010)$ & $0.067(0.019)$ & $0.165(0.028)$ & $0.385(0.040)$ & $0.368(0.040)$ \\
\hline
\end{tabular}

\footnotetext{
${ }^{43} \mathrm{Si}$ bien podría pensarse que este resultado se debe a la mayor proporción de estudiantes entre los individuos de la cohorte más joven, los datos indican que menos del 4.8\% declaran estar aún estudiando.
} 
Con relación a las tasas de permanencia intergeneracional en los niveles educativos más bajos se observa que la proporción de hijos que, al igual que sus padres, no completaron el nivel primario muestra una clara tendencia decreciente en el tiempo. Esta caída resulta más pronunciada en el caso de los varones que entre las mujeres. Para el total de hijos, la tasa de permanencia en el nivel educativo más bajo asciende, en la cohorte más vieja, a $28 \%$ pero es $11 \%$, en la cohorte más joven. Una tendencia decreciente similar se aprecia entre los hijos con padres cuyo máximo nivel educativo es el primario completo. En cambio, las tasas de permanencia en el nivel secundario incompleto así como en el secundario completo no muestran cambios significativos entre la cohorte de 1949-58 y la de 1979-88. No obstante, debe mencionarse que su evolución presenta un comportamiento diferente entre los varones en comparación con las mujeres. Así, mientras el porcentaje de hijas que no completó el secundario dado que su padre tampoco lo hizo se incrementó, lo contrario se observa entre los varones. En cambio, la tasa de permanencia en el nivel secundario completo muestra una tendencia creciente en el caso de los varones pero no así entre las mujeres.

Asimismo, en las matrices del Cuadro 4 se observa que la probabilidad de completar estudios superiores crece monotónicamente con el nivel educativo del padre. Así, la proporción de hijos con padres sin estudios primarios completos, que logran concluir estudios superiores es menor al 6\%. En cambio, el porcentaje de los que, teniendo un padre con un nivel educativo mayor al primario incompleto, realizan el mismo movimiento ascendente supera ese valor en todos los casos. Además, la probabilidad de transición de los dos niveles educativos más bajos al más alto no se modificó significativamente a lo largo del tiempo.

A partir de las matrices de transición es posible computar un conjunto de índices que resultan de utilidad para resumir algunas características relevantes de los procesos de movilidad analizados. En el Cuadro 5 se reportan los índices de movilidad descendente, ascendente y de inmovilidad así como el índice de Prais-Shorrocks con sus correspondientes errores estándares. 


\section{Cuadro 5. Índices de (in)movilidad educativa intergeneracional por cohorte de nacimiento.}

\begin{tabular}{|c|c|c|c|c|c|}
\hline Cohorte & $\begin{array}{c}\text { Movilidad } \\
\text { ascendente }\end{array}$ & $\begin{array}{c}\text { Movilidad } \\
\text { descendente }\end{array}$ & Inmovilidad & Total & $\begin{array}{c}\text { Índice de } \\
\text { Shorrocks-Prais }\end{array}$ \\
\hline \multicolumn{6}{|l|}{ Todos } \\
\hline $1949-1958$ & $\begin{array}{c}0.620 \\
(0.012)\end{array}$ & $\begin{array}{c}0.070 \\
(0.007)\end{array}$ & $\begin{array}{c}0.310 \\
(0.012)\end{array}$ & 1.000 & $\begin{array}{c}0.844 \\
(0.027)\end{array}$ \\
\hline $1959-1968$ & $\begin{array}{c}0.677 \\
(0.011)\end{array}$ & $\begin{array}{c}0.064 \\
(0.006)\end{array}$ & $\begin{array}{c}0.259 \\
(0.011)\end{array}$ & 1.000 & $\begin{array}{c}0.822 \\
(0.022)\end{array}$ \\
\hline $1969-1978$ & $\begin{array}{c}0.648 \\
(0.011)\end{array}$ & $\begin{array}{c}0.115 \\
(0.007)\end{array}$ & $\begin{array}{c}0.237 \\
(0.010)\end{array}$ & 1.000 & $\begin{array}{c}0.877 \\
(0.019)\end{array}$ \\
\hline $1979-1988$ & $\begin{array}{c}0.610 \\
(0.014)\end{array}$ & $\begin{array}{c}0.149 \\
(0.010)\end{array}$ & $\begin{array}{c}0.241 \\
(0.013)\end{array}$ & 1.000 & $\begin{array}{c}0.875 \\
(0.022)\end{array}$ \\
\hline \multicolumn{6}{|l|}{ Varones } \\
\hline 1949-1958 & $\begin{array}{c}0.617 \\
(0.018)\end{array}$ & $\begin{array}{c}0.066 \\
(0.009)\end{array}$ & $\begin{array}{c}0.318 \\
(0.017)\end{array}$ & 1.000 & $\begin{array}{c}0.854 \\
(0.038)\end{array}$ \\
\hline $1959-1968$ & $\begin{array}{c}0.672 \\
(0.016)\end{array}$ & $\begin{array}{c}0.068 \\
(0.009)\end{array}$ & $\begin{array}{c}0.260 \\
(0.015)\end{array}$ & 1.000 & $\begin{array}{c}0.829 \\
(0.034)\end{array}$ \\
\hline $1969-1978$ & $\begin{array}{c}0.623 \\
(0.016)\end{array}$ & $\begin{array}{c}0.131 \\
(0.011)\end{array}$ & $\begin{array}{c}0.246 \\
(0.014)\end{array}$ & 1.000 & $\begin{array}{c}0.868 \\
(0.027)\end{array}$ \\
\hline $1979-1988$ & $\begin{array}{c}0.572 \\
(0.021) \\
\end{array}$ & $\begin{array}{r}0.158 \\
(0.015) \\
\end{array}$ & $\begin{array}{r}0.269 \\
(0.019) \\
\end{array}$ & 1.000 & $\begin{array}{r}0.894 \\
(0.032) \\
\end{array}$ \\
\hline \multicolumn{6}{|l|}{ Mujeres } \\
\hline 1949-1958 & $\begin{array}{c}0.622 \\
(0.017)\end{array}$ & $\begin{array}{c}0.074 \\
(0.009)\end{array}$ & $\begin{array}{c}0.304 \\
(0.016)\end{array}$ & 1.000 & $\begin{array}{c}0.832 \\
(0.038)\end{array}$ \\
\hline 1959-1968 & $\begin{array}{c}0.681 \\
(0.015)\end{array}$ & $\begin{array}{c}0.061 \\
(0.008)\end{array}$ & $\begin{array}{c}0.258 \\
(0.014)\end{array}$ & 1.000 & $\begin{array}{c}0.822 \\
(0.030)\end{array}$ \\
\hline $1969-1978$ & $\begin{array}{c}0.672 \\
(0.015)\end{array}$ & $\begin{array}{c}0.100 \\
(0.009)\end{array}$ & $\begin{array}{c}0.228 \\
(0.013)\end{array}$ & 1.000 & $\begin{array}{c}0.884 \\
(0.025)\end{array}$ \\
\hline $1979-1988$ & $\begin{array}{c}0.644 \\
(0.019)\end{array}$ & $\begin{array}{c}0.140 \\
(0.014)\end{array}$ & $\begin{array}{c}0.216 \\
(0.017) \\
\end{array}$ & 1.000 & $\begin{array}{c}0.874 \\
(0.029) \\
\end{array}$ \\
\hline
\end{tabular}

Fuente: Elaboración propia sobre la base de Latinobarómetro 2003-2013.

Nota: Entre paréntesis se reportan los errores estándares computados con la técnica de bootstrap.

El índice general de movilidad ascendente que mide la proporción de hijos que logran un nivel educativo superior al de sus padres, cualquiera sea éste, no presenta variaciones significativas entre los grupos de cohortes analizados. No obstante, su valor indica que la mayoría de los hijos (poco más del 60\%, en promedio) supera el nivel de educación de su padre. Este resultado es consistente con el obtenido por Plotno (2011) para Argentina, a partir de los datos de una encuesta específica sobre estratificación y movilidad social del 2007. La autora también observa, que, tanto en la cohorte más joven considerada como en la mayor, el proceso de movilidad educativa fue mayormente ascendente, probablemente como resultado de los cambios operados en el sistema educativo. Asimismo, resulta llamativo que el índice general de movilidad descendente, esto es, la proporción de hijos que presenta un nivel educativo inferior al 
obtenido por sus padres aumente a medida que se consideran cohortes más jóvenes. Así, mientras este índice asciende al 7\% entre los hijos de la cohorte más vieja, supera el $14 \%$ en el caso de quienes pertenecen a la cohorte más joven. Por lo tanto, la caída que se aprecia en el índice de inmovilidad (en línea con la disminución observada también en el CPE y en el CCE) no responde a un aumento de la movilidad ascendente sino que, al contrario, parece estar asociada a un crecimiento de la movilidad descendente. Esto pone de relieve algunas de las limitaciones informativas de las medidas inmovilidad intergeneracional promedio como el CPE y el CCE.

Otra medida resumen del nivel de movilidad intergeneracional es el índice de PraisShorrocks $\left(\mathrm{M}_{\mathrm{PS}}\right)^{44}$ que se define de la siguiente forma:

$$
\mathrm{M}_{\mathrm{PS}}=\frac{\mathrm{n}-\operatorname{tr}(\mathrm{P})}{\mathrm{n}-1}
$$

donde $\operatorname{tr}(\mathrm{P})$ es la traza de la matriz de transición $\mathrm{P}$, es decir, la suma de los elementos de la diagonal principal y $\mathrm{n}$ es la cantidad de estados o clases considerados (niveles educativos, en este caso). Este índice toma el valor 0, en el caso de inmovilidad perfecta y 1 , en el caso de perfecta movilidad ${ }^{45}$. Conforme se observa en el Cuadro 5, el índice $\mathrm{M}_{\mathrm{PS}}$ no muestra cambios significativos. Este resultado indica que los logros educativos de los hijos de las cohortes más jóvenes no se han tornado más independientes de la distribución de la educación de sus padres, en comparación con las cohortes más viejas. Es decir, la movilidad educativa, medida a través de este índice, no se ha incrementado a lo largo de varias generaciones.

Otros índices de movilidad relativa que resultan importantes desde el enfoque de igualdad de oportunidades son los que se reportan en el Cuadro $6^{46}$. Estos índices miden la probabilidad que tiene un hijo de alcanzar un determinado nivel educativo dado que su padre lo ha completado con relación a la probabilidad que tiene otro de lograrlo cuando su padre presenta un nivel de educación inferior. Es decir, son medidas de oportunidades relativas que surgen de las matrices de transición (Daouli et al., 2010).

\footnotetext{
44 Varios estudios sobre MEI computan este índice (Checchi et al., 1999, Daouoli et al, 2010, Tansel, 2011, Ranasinghe, 2015, entre otros).

${ }^{45}$ En realidad, el máximo valor del índice de Shorrocks es n/(n-1), sin embargo, en la mayoría de los casos es poco probable que $\mathrm{M}_{\mathrm{PS}}>1$ (Ranasinghe, 2015).

${ }^{46}$ En su estudio empírico Heineck y Riphan (2007) proponen estos índices a fin examinar los niveles y evolución de MEI en Alemania. Daouli et al. (2010) también computan estos indicadores para Grecia.
} 


\section{Cuadro 6. Ratio entre la probabilidad de los hijos de alcanzar un nivel educativo condicional al nivel educativo del padre por cohorte de nacimiento.}

\begin{tabular}{|c|c|c|c|c|}
\hline Cohorte & $\begin{array}{c}\operatorname{Prob}\left(\mathrm{H}^{\mathrm{UC}} \mid \mathbf{P}^{\mathrm{UC}}\right) / \\
\operatorname{Prob}\left(\mathbf{H}^{\mathrm{UC}} \mid \mathbf{P}^{\mathrm{PC}}\right)\end{array}$ & $\begin{array}{c}\operatorname{Prob}\left(\mathbf{H}^{\mathrm{UC}} \mid \mathbf{P}^{\mathrm{UC}}\right) / \\
\operatorname{Prob}\left(\mathbf{H}^{\mathrm{UC}} \mid \mathbf{P}^{\mathrm{SC}}\right)\end{array}$ & $\begin{array}{c}\operatorname{Prob}\left(\mathbf{H}^{\mathrm{SC}} \mid \mathbf{P}^{\mathrm{SC}}\right) / \\
\operatorname{Prob}\left(\mathbf{H}^{\mathrm{SC}} \mid \mathbf{P}^{\mathrm{PC}}\right)\end{array}$ & $\begin{array}{c}\operatorname{Prob}\left(\mathbf{H}^{\mathrm{SC}} \mid \mathbf{P}^{\mathrm{SC}}\right) / \\
\operatorname{Prob}\left(\mathbf{H}^{\mathrm{SC}} \mid \mathbf{P}^{\mathrm{SI}}\right)\end{array}$ \\
\hline \multicolumn{5}{|l|}{ Todos } \\
\hline \multirow[t]{2}{*}{$1949-1958$} & 4.923 & 1.755 & 1.359 & 1.137 \\
\hline & $(0.818)$ & $(0.313)$ & $(0.183)$ & $(0.308)$ \\
\hline \multirow[t]{2}{*}{$1959-1968$} & 4.272 & 1.385 & 1.027 & 0.815 \\
\hline & $(0.601)$ & $(0.188)$ & $(0.124)$ & $(0.160)$ \\
\hline \multirow[t]{2}{*}{$1969-1978$} & 4.014 & 1.431 & 0.985 & 0.906 \\
\hline & $(0.516)$ & $(0.163)$ & $(0.096)$ & $(0.146)$ \\
\hline \multirow[t]{2}{*}{$1979-1988$} & 4.498 & 1.569 & 1.176 & 1.099 \\
\hline & $(0.897)$ & $(0.246)$ & $(0.130)$ & $(0.187)$ \\
\hline \multicolumn{5}{|l|}{ Varones } \\
\hline \multirow[t]{2}{*}{$1949-1958$} & 5.751 & 1.444 & 1.166 & 0.855 \\
\hline & (1.633) & $(0.393)$ & $(0.268)$ & $(0.362)$ \\
\hline \multirow[t]{2}{*}{$1959-1968$} & 5.061 & 1.175 & 1.005 & 1.015 \\
\hline & (1.382) & $(0.289)$ & $(0.169)$ & $(0.312)$ \\
\hline \multirow[t]{2}{*}{$1969-1978$} & 4.876 & 1.459 & 1.012 & 0.854 \\
\hline & (1.106) & $(0.269)$ & $(0.141)$ & $(0.183)$ \\
\hline \multirow{2}{*}{$1979-1988$} & 5.624 & 2.546 & 1.373 & 0.920 \\
\hline & $(1.627)$ & $(0.605)$ & $(0.214)$ & $(0.203)$ \\
\hline \multicolumn{5}{|l|}{ Mujeres } \\
\hline \multirow[t]{2}{*}{$1949-1958$} & 4.335 & 2.124 & 1.502 & 1.396 \\
\hline & $(0.888)$ & $(0.536)$ & $(0.246)$ & $(0.493)$ \\
\hline \multirow[t]{2}{*}{$1959-1968$} & 3.651 & 1.428 & 1.058 & 0.695 \\
\hline & $(0.585)$ & $(0.238)$ & $(0.186)$ & $(0.179)$ \\
\hline \multirow[t]{2}{*}{$1969-1978$} & 3.825 & 1.437 & 0.964 & 0.976 \\
\hline & $(0.583)$ & (0.199) & $(0.132)$ & $(0.238)$ \\
\hline \multirow[t]{2}{*}{$1979-1988$} & 3.493 & 0.996 & 1.004 & 1.250 \\
\hline & $(1.000)$ & $(0.227)$ & $(0.161)$ & $(0.321)$ \\
\hline
\end{tabular}

Nota: H: hijos, P: padres, UC: superior completo, SC: secundario completo, SI: secundario incompleto, PC: primario completo. Entre paréntesis se reportan los errores estándares computados con el método delta.

Fuente: Elaboración propia sobre la base de Latinobarómetro 2003-2013.

En el Cuadro 6 se reportan los índices referidos al nivel superior y al nivel secundario. Así, en todos los grupos de cohortes considerados, la ratio $\operatorname{Prob}\left(\mathrm{H}^{\mathrm{UC}} \mid \mathrm{P}^{\mathrm{UC}}\right) / \operatorname{Prob}\left(\mathrm{H}^{\mathrm{UC}} \mid \mathrm{P}^{\mathrm{PC}}\right)$ indica que la probabilidad de un hijo de lograr el nivel educativo superior dado que su padre lo alcanzó supera en más de 4 veces la probabilidad que tiene otro de hacerlo cuando su padre sólo completó el nivel primario. Esta ratio asciende a más de 5 en el caso de los hijos varones. Las desigualdades en oportunidades educativas se reducen cuando se compara a hijos cuyos padres tienen estudios superiores con aquellos con padres que completaron el nivel secundario. Así, conforme a la ratio $\operatorname{Prob}\left(\mathrm{H}^{\mathrm{UC}} \mid \mathrm{P}^{\mathrm{UC}}\right) / \operatorname{Prob}\left(\mathrm{H}^{\mathrm{UC}} \mid \mathrm{P}^{\mathrm{SC}}\right)$ un hijo con un padre con estudios superiores tiene 1.4 veces más probabilidades de completar el máximo nivel educativo que aquel cuyo padre completó el nivel secundario. Las probabilidades condicionales de 
completar el nivel secundario presentan diferencias menores entre los hijos con un padre que terminó el secundario y aquellos cuyos padres sólo completaron el primario o los hijos de padres que tienen estudios secundarios incompletos. No obstante, en el caso de las mujeres de la cohorte más vieja la ratio $\operatorname{Prob}\left(\mathrm{H}^{\mathrm{SC}} \mid \mathrm{P}^{\mathrm{SC}}\right) / \operatorname{Prob}\left(\mathrm{H}^{\mathrm{SC}} \mid \mathrm{P}^{\mathrm{PC}}\right)$ asciende a 1.5. En tanto que la ratio $\operatorname{Prob}\left(\mathrm{H}^{\mathrm{SC}} \mid \mathrm{P}^{\mathrm{SC}}\right) / \operatorname{Prob}\left(\mathrm{H}^{\mathrm{SC}} \mid \mathrm{P}^{\mathrm{SI}}\right)$ indica que las hijas de esa cohorte de nacimiento con un padre que terminó el nivel secundario tiene 1.4 veces más probabilidades de conseguirlo que aquellas cuyo padre no completó este nivel educativo. Sin embargo, para el nivel secundario, las probabilidades condicionales y las ratios sugieren, en general, una mayor igualdad de oportunidades relativas, particularmente entre las cohortes más jóvenes. Asimismo, todas las ratios analizadas muestran una leve tendencia decreciente entre las mujeres, no así en el caso de los varones. No obstante, ninguna de las variaciones observadas entre las cohortes es significativa.

\subsection{Evaluación de los procesos de movilidad educativa intergeneracional desde el enfoque de igualdad de oportunidades}

En esta sección se examinan los procesos de movilidad educativa intergeneracional desde el enfoque EOP, a partir del índice OV propuesto recientemente por Anderson et al. (2014). Esta medida permite evaluar la existencia de EOP, en cada una de las cohortes de nacimiento consideradas. Conforme con la ecuación (10), esto puede realizarse comparando cada una de las densidades conjuntas observadas del nivel educativo de hijos y padres (que se representan en el panel de la izquierda del Gráfico 3, para la cohorte más vieja y la más joven), con la densidad conjunta que existiría en teoría bajo el supuesto de EOP o de independencia del nivel educativo de padres e hijos (que se muestran en el panel de la derecha del Gráfico 3). En la medida en que la densidad conjunta observada y la teórica sean similares (distintas), el índice OV se acercará a 1 (0). A lo largo del período analizado, las densidades conjuntas observadas y las estimadas bajo la hipótesis de independencia entre el nivel educativo de padres e hijos, se hicieron más cercanas conforme con los índices OV computados para las 
distintas cohortes de nacimiento ${ }^{47}$. Así, esta medida se incrementó, entre la cohorte más vieja y la más joven, un 33\% en el total de hijos, un $28 \%$ entre los varones y un $36 \%$ en el caso de las mujeres. Esto implica una mejora en términos de EOP, para las generaciones más jóvenes en comparación con las mayores.

\section{Gráfico 3. Densidades de probabilidad conjunta del nivel educativo de padres e hijos, observadas y bajo la hipótesis de EOP o independencia por cohorte de nacimiento.}
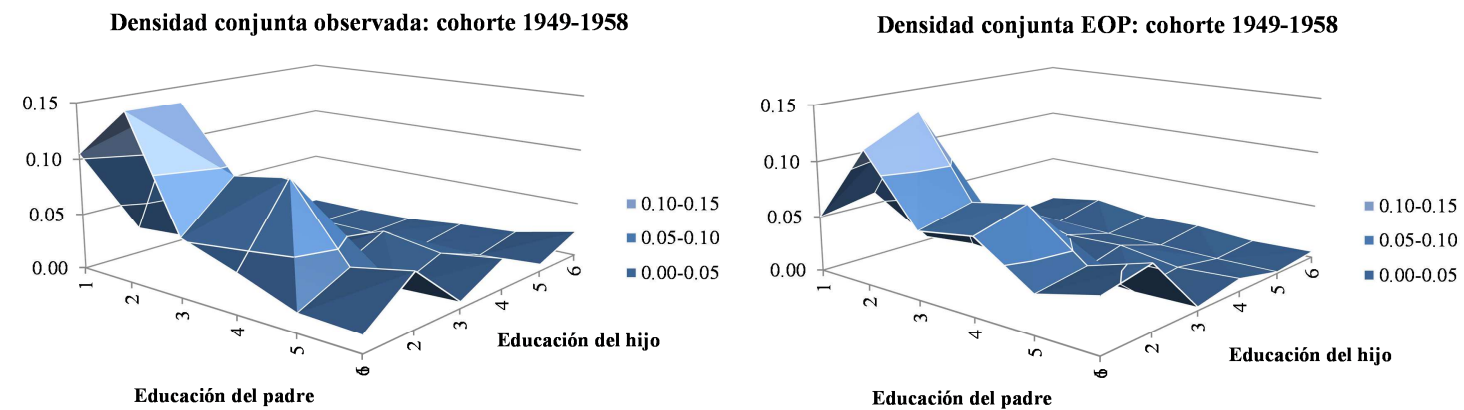

Densidad conjunta observada: cohorte 1979-1988

Densidad conjunta EOP: cohorte 1979-1988
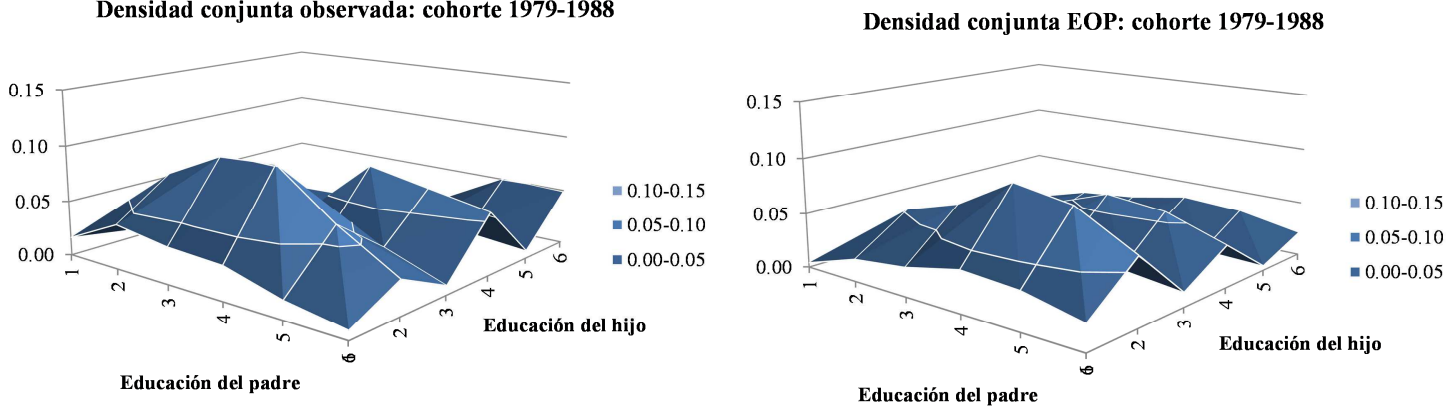

Nota: 1: primario incompleto, 2: primario completo, 3: secundario incompleto, 4: secundario completo, 5: superior incompleto, 6: superior completo.

Fuente: Elaboración propia sobre la base de Latinobarómetro 2003-2013.

No obstante, el índice OV general no permite determinar qué grupo socioeconómico se benefició desde el punto de vista de la EOP. Esto implica evaluar la denomina hipótesis QEO que, en términos generales, establece que la densidad condicional del logro de los hijos que pertenecen al grupo socioeconómico más desaventajado debería ser cercana a la densidad marginal del logro de los hijos, en comparación con los hijos de grupos de mayor status socioeconómico. El índice $O V$ de la ecuación (11) permite testear esta hipótesis porque mide el grado de solapamiento (overlap) entre las dos densidades para cada grupo socioeconómico de origen definido,

\footnotetext{
${ }^{47}$ Ver la última columna del Cuadro 7, del Cuadro A4 y del Cuadro A5 del Anexo.
} 
en este caso, a partir del nivel educativo paterno. Si el nivel educativo de los hijos y el del padre son independientes entonces el índice $O V$ presentará valores cercanos a 1 , indicando el cumplimiento de la hipótesis de EOP para ese grupo. En la medida en que el nivel educativo de los hijos dependa más de la educación de sus padres, de manera que la densidad condicional se aleje de la densidad marginal de la educación de los hijos, el índice registrará un valor substancialmente menor que 1 y cercano a 0. Entonces cuanto más alejada de 1 se encuentre esta medida para un grupo particular, menor será la EOP entre los hijos que lo conforman. De esta manera, el índice $O V$ permite no sólo evaluar la existencia de igualdad de oportunidades sino también determinar cuán alejado se encuentra un grupo particular de una situación de EOP en la que el resultado obtenido por los hijos no depende del logro de sus padres. Esta medida facilita también las comparaciones temporales de los procesos de movilidad representados por toda la distribución de densidad condicional del nivel educativo de los hijos dado el nivel de educación del padre. En efecto, el índice $O V$ permite resumir en una estadística las diferencias existentes, en términos de EOP o QOP, entre los procesos de movilidad intergeneracional experimentados por distintas cohortes de nacimiento. De esta forma, es posible aportar evidencia de la existencia de una tendencia hacia o en contra de la EOP.

En el Cuadro 7 se presentan los índices $O V$ computados conforme con la ecuación (11) para todos los hijos de 25 a 64 años por cohorte de nacimiento y nivel educativo paterno, debajo de ellos se muestran las magnitudes de las diferencias computadas entre los índices de los distintos grupos de cohortes. Los Cuadros A4 y A5 del Anexo contienen los índices $O V$ correspondientes a los varones y a las mujeres respectivamente. En primer lugar, se aprecia una tendencia general hacia una mayor igualdad de oportunidades educativas entre los hijos de ambos sexos cuyos padres tienen nivel secundario completo y estudios superiores (incompletos o completos). Esto se deduce del crecimiento que se observa en el índice $O V$ a medida que nos movemos de la cohorte más vieja hacia las más jóvenes, particularmente en el caso de los varones. A partir de las diferencias computadas en esta medida entre la cohorte más vieja y la más joven, las mayores ganancias, en términos de EOP, se observa entre los hijos con padres que terminaron el nivel secundario y aquellos con estudios superiores incompletos. Así, en el caso de los varones cuyos padres tienen estudios secundarios 
completos, el índice $O V$ de la cohorte más joven es un $47 \%$ más elevado que el estimado para la cohorte más vieja. Conforme a los $\mathrm{Z}$ test computados, esta diferencia resulta estadísticamente significativa. Entre las mujeres de la cohorte 1969-78 (la segunda más joven) también se aprecian ganancias estadísticamente significativas en términos de EOP, en comparación con las cohortes más viejas, entre las que provienen de familias con un padre con estudios superiores incompletos.

\section{Cuadro 7. Índices OV por nivel educativo del padre y cohorte de nacimiento. Varones y mujeres}

\begin{tabular}{|c|c|c|c|c|c|c|c|c|}
\hline \multirow{2}{*}{$\begin{array}{l}\text { Cohorte de } \\
\text { nacimiento }\end{array}$} & \multicolumn{6}{|c|}{ Nivel educativo del padre } & \multirow{2}{*}{\multicolumn{2}{|c|}{ Total }} \\
\hline & $\begin{array}{c}\text { Primario } \\
\text { incompleto }\end{array}$ & $\begin{array}{l}\text { Primario } \\
\text { completo }\end{array}$ & $\begin{array}{l}\text { Secundario } \\
\text { incompleto }\end{array}$ & $\begin{array}{c}\text { Secundario } \\
\text { completo }\end{array}$ & $\begin{array}{c}\text { Superior } \\
\text { incompleto }\end{array}$ & $\begin{array}{l}\text { Superior } \\
\text { completo }\end{array}$ & & \\
\hline \multirow{2}{*}{ [1] 1949-58 } & 0.764 & 0.895 & 0.766 & 0.582 & 0.444 & 0.448 & 0.534 & \\
\hline & $(0.038)$ & $(0.119)$ & $(0.245)$ & $(0.031)$ & $(0.174)$ & $(0.380)$ & $(0.046)$ & \\
\hline \multirow[t]{2}{*}{ [2] 1959-68 } & 0.749 & 0.903 & 0.732 & 0.639 & 0.508 & 0.536 & 0.570 & \\
\hline & $(0.030)$ & $(0.037)$ & $(0.257)$ & $(0.123)$ & $(0.311)$ & $(0.194)$ & $(0.034)$ & \\
\hline \multirow[t]{2}{*}{ [3] 1969-78 } & 0.727 & 0.891 & 0.890 & 0.751 & 0.754 & 0.569 & 0.626 & \\
\hline & $(0.043)$ & $(0.064)$ & $(0.047)$ & $(0.169)$ & $(0.325)$ & $(0.094)$ & $(0.021)$ & \\
\hline \multirow[t]{2}{*}{ [4] 1979-88 } & 0.728 & 0.848 & 0.866 & 0.823 & 0.679 & 0.642 & 0.712 & \\
\hline & $(0.038)$ & $(0.084)$ & $(0.337)$ & $(0.131)$ & $(0.314)$ & $(0.257)$ & $(0.060)$ & \\
\hline \multicolumn{9}{|c|}{ Diferencias entre cohortes } \\
\hline \multirow[t]{2}{*}[4]{$-[1]$} & -0.037 & -0.047 & 0.100 & $0.241 \quad * *$ & 0.234 & 0.194 & 0.179 & $* * *$ \\
\hline & $(0.163)$ & $(0.348)$ & $(0.576)$ & $(0.041)$ & $(0.445)$ & $(0.368)$ & $(0.000)$ & \\
\hline \multirow[t]{2}{*}{ [4]-[3] } & 0.001 & -0.044 & -0.024 & 0.072 & -0.075 & 0.072 & 0.087 & * \\
\hline & $(0.991)$ & $(0.316)$ & $(0.943)$ & $(0.190)$ & $(0.748)$ & $(0.823)$ & $(0.071)$ & \\
\hline \multirow[t]{2}{*}[3]{$-[2]$} & -0.022 & -0.012 & 0.158 & 0.112 & 0.246 & 0.033 & 0.056 & \\
\hline & $(0.730)$ & $(0.734)$ & $(0.545)$ & $(0.195)$ & $(0.247)$ & $(0.905)$ & $(0.266)$ & \\
\hline \multirow[t]{2}{*}[2]{$-[1]$} & -0.015 & 0.009 & -0.034 & 0.057 & 0.064 & 0.088 & 0.036 & \\
\hline & $(0.797)$ & $(0.923)$ & $(0.863)$ & $(0.589)$ & $(0.835)$ & $(0.796)$ & $(0.639)$ & \\
\hline \multirow[t]{2}{*}[4]{$-[2]$} & -0.021 & -0.056 & 0.134 & $0.184 \quad * * *$ & 0.171 & 0.105 & 0.142 & \\
\hline & $(0.746)$ & $(0.365)$ & $(0.429)$ & $(0.000)$ & $(0.104)$ & $(0.682)$ & $(0.118)$ & \\
\hline \multirow[t]{2}{*}{ [3]-[1] } & -0.037 & -0.003 & 0.124 & 0.169 & 0.309 & 0.121 & 0.092 & $* *$ \\
\hline & $(0.562)$ & $(0.956)$ & $(0.611)$ & $(0.287)$ & $(0.377)$ & $(0.786)$ & $(0.011)$ & \\
\hline
\end{tabular}

Nota: en el primer panel debajo los índices OV entre paréntesis se reportan los errores estándares computados con la técnica de bootstrap y en el segundo panel, debajo de las diferencias entre los índices OV se encuentra el $p$-value correspondiente a la estadística Z. *** p-value $<0.01, * *$ p-value $<0.05, *$ p-value $<0.1$.

Fuente: Elaboración propia sobre la base de Latinobarómetro 2003-2013.

Por otra parte, debe destacarse la falta de ganancias, en términos de EOP, a lo largo de las distintas cohortes, entre los hijos de padres con los niveles educativos más bajos. Esto podría sugerir que las medidas de política tendientes a igualdad oportunidades educativas no fueron efectivas en aumentar, a lo largo de las distintas generaciones, la igualdad de oportunidades educativas entre los hijos más desaventajados. No obstante, es importante notar también que, en cada una de los 
grupos de cohortes considerados, los niveles de EOP, medidos por el índice OV, entre los hijos con padres que tienen los tres niveles educativos más bajos superan los observados entre aquellos cuyos padres completaron los estudios superiores. Es decir, en general, durante varias generaciones, la movilidad educativa intergeneracional entre los primeros se mantuvo más cercana a una situación de independencia que en el caso de los últimos. Este resultado es consistente con el derivado a partir del análisis de regresión, concretamente de la relación negativa observada entre la educación del padre y la varianza del modelo en cada cohorte de nacimiento. No obstante, estas estimaciones también mostraban un incremento significativo del grado de heteroscedasticidad entre la cohorte más joven y la más vieja sugiriendo una mejora en términos de EOP entre los hijos de padres con bajos niveles educativos. Sin embargo, eso no se observa a partir del índice $O V$, al menos no en el caso de los hijos cuyos padres tienen niveles educativos inferiores al secundario completo. Esto da cuenta de las limitaciones del enfoque de regresión para evaluar las implicancias de los niveles de movilidad intergeneracional observados en términos de EOP.

Por último, en general, en cada una de las cohortes de nacimiento y para los distintos niveles educativos del padre, no se observan diferencias significativas por género en los índices $O V$. Si bien, en cohorte más joven de los $80 \mathrm{~s}$, el índice OV general así como el correspondiente a hijos con padres que completaron estudios superiores es mayor entre las mujeres que en el caso de los varones, estas diferencias no resultan estadísticamente significativas. Por tanto, estos resultados sugieren que los niveles de MEI son similares entre varones y mujeres en términos de EOP.

\section{Conclusiones}

El objetivo principal de esta investigación fue analizar las tendencias de la movilidad educativa intergeneracional en Argentina durante las 3 décadas que separan a las cohortes que nacieron en los 50s de aquellas que nacieron en los $80 \mathrm{~s}$. El análisis se centró primordialmente en evaluar la evolución de la MEI desde el enfoque de igualdad de oportunidades. La pregunta central que se intentó responder es en qué medida los cambios observados en el proceso de movilidad intergeneracional modificaron los niveles de igualdad de oportunidades, particularmente entre aquellos que pertenecen a 
los grupos socioeconómicos más desaventajados. Este análisis constituye la principal contribución del capítulo a la literatura existente. Con este fin, se implementó una nueva técnica propuesta Anderson et al. (2014) que consiste en medir el grado de solapamiento (overlap) entre la densidad condicional de la educación de los hijos dada la educación de los padres y la densidad teórica correspondiente a una situación de EOP o independencia entre las dos variables. Esta técnica tiene varias ventajas sobre otros métodos existentes, entre ellas, ofrecer una medida - el índice OV - que permite no sólo evaluar la existencia de igualdad de oportunidades sino también los progresos que la sociedad realizada en esta dimensión. Específicamente, permite examinar si las generaciones más jóvenes tuvieron una mayor oportunidad de obtener un mejor resultado educativo que las generaciones anteriores. Además, a partir de esta técnica es posible evaluar la denominada hipótesis de QEO, es decir, si los hijos que más se beneficiaron desde el punto de vista de la EOP son aquellos que pertenecen a los grupos más desaventajados en función de los bajos niveles educativos de sus padres.

Los resultados obtenidos de los enfoques paramétrico y no paramétrico implementados para caracterizar el proceso de MEI y sus cambios temporales permitieron derivar varias conclusiones. En primer lugar, las estimaciones del modelo de regresión intergeneracional mostraron una disminución significativa en los niveles promedio de persistencia educativa intergeneracional, medida a partir del coeficiente de regresión de la educación de los padres (aquí denominado CPE), a lo largo de las cohortes de nacimiento, en los últimos 30 años. Esta caída se aprecia tanto entre varones como entre mujeres. Sin embargo, no se observa una tendencia decreciente similar a partir de la correlación educativa intergeneracional. Esta discrepancia entre las dos medidas responde a las diferencias en la evolución de la dispersión en los logros educativos en las dos generaciones. Un resultado similar es obtenido por Hertz et al. (2007) para un amplio conjunto de países, por Daude y Robano (2015) para 18 países latinoamercanos así como por Azam y Bhatt (2014) para la India, entre otros ${ }^{48}$.

En segundo lugar, a partir de las matrices de transición educativa se observaron importantes asimetrías en el proceso de movilidad intergeneracional con una mayor

\footnotetext{
48 Aaronson y Mazumder (2008) encuentran la misma discrepancia entre el coeficiente de la regresión intergeneracional y el coeficiente de correlación al analizar las tendencias en la movilidad intergeneracional de ingresos en los Estados Unidos.
} 
persistencia de la educación entre los padres y los hijos que se encuentran en el nivel educativo más alto. No obstante, esta tasa de persistencia en el nivel superior completo disminuyó entre la cohorte más vieja y la más joven, como resultado de un aumento en las tasas de movilidad descendente. Asimismo, las tasas de permanencia intergeneracional en los niveles educativos más bajos también muestran una tendencia decreciente. En cambio, las tasas de persistencia en el nivel secundario incompleto y completo no muestran cambios significativos entre la cohorte de 1949-58 y la de 197988. Además, se observó que la probabilidad de completar estudios superiores crece, en todas las cohortes de nacimiento, con el nivel educativo del padre. Los índices de inmovilidad intergeneracional, computados a partir de las matrices de transición, sugieren una caída en los niveles promedio de persistencia educativa, en línea con lo observado a partir del CPE. Sin embargo, esto se explica por un aumento en el índice de movilidad descendente. Es decir, no responde a un crecimiento en las tasas de movilidad ascendente.

Por otra parte, la evaluación de los procesos de MEI desde el enfoque igualdad de oportunidades, a partir de la técnica de overlap de Anderson et al. (2014), sugiere una mejora en los niveles promedio de EOP entre las generaciones más jóvenes. No obstante, según los índices $O V$ estimados, esta ganancia en términos de EOP, a lo largo de las cohortes de nacimiento, se observa en mayor medida entre los hijos cuyos padres tienen nivel secundario completo y estudios superiores. Más aún, es notable la falta de mejora de los niveles de EOP, medidos por el índice $O V$, entre los hijos de padres con los niveles educativos más bajos. No obstante, también se aprecia, en general, durante varias generaciones, que la movilidad educativa intergeneracional entre los hijos con padres que tienen los tres niveles educativos más bajos se mantuvo más cercana a una situación de independencia o EOP que en el caso de aquellos cuyos padres completaron los estudios superiores. Pero, la relativa estabilidad del índice OV entre los hijos del grupo educativo de origen más desaventajado sugiere que las políticas públicas no fueron lo suficientemente efectivas para mejorar la igualdad de oportunidades educativas entre ellos.

Por último, una de las próximas preguntas a examinar, que surgen de la investigación de este capítulo, se relaciona con los factores que pueden explicar la caída observada en la persistencia educativa intergeneracional y el aumento en los niveles 
generales de igualdad de oportunidades educativas entre la cohorte más joven considerada en comparación con la mayor. De acuerdo con el modelo teórico de Solon (2004), la inversión pública en el capital humano de niños y adolescentes es un factor central en el proceso de transmisión intergeneracional del status socioeconómico. El análisis de los efectos de esta variable sobre la movilidad intergeneracional del ingreso y de la educación en Argentina constituye el principal objetivo del Capítulo 3. 


\section{Referencias bibliográficas}

Aaronson, D. y Mazumder, B. (2008). "Intergenerational economic mobility in the United 1940 to 2000", Journal of Human Resources, 43(1), 140-172.

Aldaz-Carrol, E. y Moran, R. (2001). "Escaping the poverty trap in Latin America: the role of family factors", Cuadernos de Economía, 38(114), 155-190.

Andersen, L. (2001). "Social mobility in Latin America: links with adolescent schooling”, Inter-American Development Bank, IADB Research Network Working Paper R-433.

Anderson, G. y Leo, T. W. (2015). "Quantifying the progress of economic and social justice: charting changes in equality of opportunity in the United States 1960-2000", Journal of Human Development and Capabilities, 1-29.

Anderson, G., Fruehauf, T., Pittau, M. G. y Zelli, R. (2015). "Evaluating progress toward an equal opportunity goal: assessing the German educational reforms of the first decade of the $21^{\text {st }}$ century", Departamento de Economía, Universidad de Toronto, Working paper No. 552.

Anderson, G., Leo, T. W. y Muelhaupt, R. (2014). "Measuring advances in equality of opportunity: The changing gender gap in educational attainment in Canada in the last half century", Social Indicators Research, 119(1), 73-99.

Anderson, G., Linton, O. y Whang, Y. J. (2012). "Nonparametric estimation and inference about the overlap of two distributions", Journal of Econometrics, 171(1), 123.

Anderson G. J., Ge, Y. y Leo, T. W. (2010). "Distributional overlap: simple multivariate parametric and non-parametric tests for alienation, convergence and general distributional difference issues", Econometric Reviews, 29(3), 1532-4168.

Azam, M. y Bhatt. V. (2014). "Like Father, Like Son? Intergenerational education mobility in India”, Institute for the Study of Labor, IZA Discussion Paper Series No. 6549.

Becker, G. S. y Tomes, N. (1979). “An equilibrium theory of the distribution of income and intergenerational mobility", Journal of Political Economy, 87(6), 1153-89.

Becker, G. S. y Tomes, N. (1986). "Human capital and the rise and fall of families", Journal of Labor Economics, 4(3), pt. 2, S1-S39. 
Behrman, J. N., Birdsall, N. y Szekely, M. (1999). "Intergenerational mobility in Latin America: deeper markets and better schools make a difference”. En Birdsall, N. y Graham, C., eds., New Markets, New Opportunities? Economic and social mobility in a changing world. Washington, DC: Brookings Institution.

Behrman, J. R, Gaviria, A. y Székeley, M. (2001). "Intergenerational mobility in Latin America", Economia, 2(1), 1-31.

Benabou, R. y Ok, E. A. (2001). "Mobility as progressivity: ranking income process according to equality of opportunity", The National Bureau of Economic Research, NBER working paper series No. 8431.

Binder, M. y Woodruff, C. (2002). "Inequality and intergenerational mobility in schooling: the case of Mexico", Economic Development and Cultural Change, 50(2), 249-267.

Bingley, P., Kaare, C. y Jensen, V. M. (2009). "Parental schooling and child development: learning from twin parents", Danish National Centre for Social Research Social Policy and Welfare Services Working Paper No. 07.

Black, S. y Devereux, P. (2011). "Recent developments in intergenerational mobility." En Ashenfelter, O. y Card, D., eds., Handbook of Labor Economics. Amsterdam: North-Holland Press.

Blanden, J. (2013). "Cross-country rankings in intergenerational mobility: a comparison of approaches from economics and sociology", Journal of Economic Surveys, 27(1), $38-73$.

Blanden, J. y Machin, S. (2004). "Educational inequality and the expansion of UK higher education", Scottish Journal of Political Economy, 51(2), 230-249.

Blanden, J., Gregg, P. y Machin, S. (2005). "Intergenerational in Europe and North America", A report supported by the Sutton trust, Centre for Economic Performance, London School of Economics.

Blanden, J., Gregg, P. y Macmillan, L. (2007). “Accounting for intergenerational income persistence: noncognitive skills, ability and education", Economic Journal, 117(519), C43-C60.

Brunori, P., Ferreira, F. H. G. y Peragine, V. (2013). "Inequality of opportunity, income inequality and economic mobility: some international comparisons", en E. Paus, ed., Getting development right. Structural transformation, inclusion, and sustainability in the post-crisis era, New York: Palgrave Macmillan US, 85-115. 
Carneiro, P., Meghir, C. y Parey, M. (2013). "Maternal education, home environments and the development of children and adolescents", Journal of the European Economic Association, 11(s1), 123-160.

Cartagena, K. (2003). "Educación y movilidad intergeneracional en Colombia, 19291996", Estudios Económicos de Desarrollo Internacional, 3(2), 27-66.

Casal, M. P. y Paz Terán, C. M. (2012). “An approach to intergenerational persistence in education in Argentina: 1970-2010", Anales de la Asociación Argentina de Economía Política (AAEP), Trelew.

Castañeda, T. y Aldaz-Carroll, E. (1999). "The intergenerational transmission of poverty: some causes and policy implications", Banco Interamericano de Desarrollo, Washington D. C.

Checchi, D., Fiorio, C. y Leonardi, M. (2008). "Intergenerational persistence of educational attainment in Italy", Institute for the Study of Labor, IZA Discussion Paper Series No. 3622.

Checchi D., Ichino, A. y Rustichini, A. (1999) "More equal but less mobile? Educational financing and intergenerational mobility in Italy and in the US", Journal of Public Economics, 74(3), 351-393.

Checchi, D., Fiorio, C. y Leonardi, M. (2013). "Intergenerational persistence of educational attainment in Italy", Economics Letters, 118(1), 229-232.

Conconi, A., Cruces, G., Olivieri, S. y Sánchez, R. (2007). "E pur si muove? movilidad, pobreza y desigualdad en América Latina”, Económica, LIV(1-2), 121-159.

Cruces, G. (2008). "Tópicos de Economía distributiva", Maestría en Economía, Facultad de Ciencias Económicas, Universidad Nacional de La Plata, Material de clases no publicados.

Dahan, M. y Gaviria, A. (2001). "Sibling correlations and intergenerational mobility in Latin America", Economic Development y Cultural Change, 49(3), 537-54.

Daouli, J, Demoussis, M. y Giannalopoulos, N. (2010). "Mothers, fathers and daughters: Intergenerational transmission of education in Greece", Economics of Education Review, 29(1), 83-93.

Daude, C. y Robano, V. (2015). "On intergenerational (im)mobility in Latin America", Latin American Economic Review, 24(9), 2-29. 
Di Paolo (2012). "Parental education and family characteristics: educational opportunities across cohorts in Italy and Spain”, Revista de Economía aplicada, 20(58), 119-146.

Dubet, F. (2011). Repensar la justicia social. Buenos Aires: Siglo XXI Editores.

Dustmann, C. (2004). "Parental background, secondary school track choice, and wages”, Oxford Economic Papers, 56(2), 209-230.

Fernández, A. G. (2006). "Alternative measures of intergeneracional social mobility in Argentina", Anales de la Asociación Argentina de Economía Política (AAEP), Buenos Aires.

Ferreira, F. H. G., Messina, J., Rigolini, J., López-Calva, L-F., Lugo, M. A. y Vakis, R. (2013). Economic mobility and the rise of the Latin American middle class. Washington, DC: World Bank.

FIEL (2008). La igualdad de oportunidades en la Argentina. Movilidad intergeneracional en los 2000, Buenos Aires: Temas grupo editorial.

Formby, J. P., Smith, W. J. y Zheng, B. (2004). "Mobility measurement, transition matrices and statistical inference", Journal of Econometrics 120(1), 181-205.

Gaviria, A. (2006). "Movilidad social en América Latina: realidades y percepciones", mimeo, Facultad de Economía, Universidad de los Andes.

Gandelman, N. y Robano, V. (2014). "Intergenerational mobility and entrepreneurship in Uruguay", Latin American Journal of Economics, 51(2), 95-226.

Gasparini, L. (2007). "Monitoring the socio-economic conditions in Argentina 19922006", CEDLAS, Centro de Estudios Distributivos, Laborales y Sociales, Facultad de Ciencias Económicas, Universidad Nacional de La Plata.

Gasparini, L. y Marchionni, M. (2015). "Bridging gender gaps? The rise and deceleration of female labor force participation in latin america: An overview", Documento de trabajo No. 185, CEDLAS, Facultad de Ciencias Económicas, Universidad Nacional de La Plata.

Güell, M., Rodríguez-Mora, S. y Telmer, C. (2007), "Intergenerational mobility and the informative content of surnames", C.E.P.R. Discussion paper No. 6316.

Haegeland, T., Kirkeboen, L. J., Raaum, O. y Salvanes, K. G. (2010). "Why children of college graduates outperform their schoolmates: A study of cousins and adoptees", Institute for the Study of Labor, IZA Discussion Paper No. 5369. 
Hanslin, S., Winkelmann, R. y Cattaneo, A. (2007). "The apple falls increasingly far: Parent-child correlation in schooling and the growth of post-secondary education in Switzerland", Swiss Journal of Economics and Statistics, 143(2), 133-152.

Heineck, G. y Riphahn, R. T. (2007). "Intergenerational transmission of educational attainment in Germany: The last five decades", Institute for the Study of Labor, IZA Discussion Paper Series No. 2985.

Hertz, T., Jayasundera, T., Praino, P., Selcuk, S., Smith, N. y Verashchagina, A. (2007). "The inheritance of educational inequality: international comparison and fifty-year trends", The B.E Journal of Economic Analysis and Policy, 7(2), 1-45.

Hirschman, A. y Rothschild, M. (1973). "The changing tolerance for income inequality in the course of economic development", The Quarterly Journal of Economics, 87(4), 544-66.

Holmlund, H. y Lindahl, M. y Plug, E. (2011). "The causal effect of partens' schooling on children's schooling: A comparison of estimation methods", Journal of Economic Literature, 49(3), 615-651.

Jorrat, J. R. (2010). "Logros educacionales y movilidad educacional intergeneracional en Argentina", Desarrollo Económico, 49(196), 573-604.

Jorrat, J. R. (2000). Estratificación social y movilidad: un estudio del Área Metropolitana de Buenos Aires. Tucumán: Editorial de la Universidad Nacional de Tucumán.

Lefranc, A., Pistolesi, N. y Trannoy, A. (2008). "Inequality of opportunity vs. Inequality of outcomes: are western societies all alike?", Review of Income and Wealth, 54(4), 513-546.

Lefranc, A., Pistolesi, N., y Trannoy, A. (2009). "Equality of opportunity and luck: definitions and testable conditions, with an application to income in France", Journal of Public Economics, 93(11-12), 1189-1207.

Machin, S. y Vignoles, A. (2004). "Educational Inequality: The Widening SocioEconomic Gap", Fiscal Studies, 25(2), 107-128.

Magejo, P., Ntuli, M. y Gwatidzo, T. (2014). "Trends in the intergenerational transmission of education among black south Africans", Institute for the Study of Labor, IZA Discussion paper series No. 8546. 
Marteleto L, Gelber, D., Hubert, C. y Salinas, V. (2012). "Educational inequalities among Latin American adolescents: continuities and changes over the 1980s, 1990s and 2000s", Research in Social Stratifcation and Mobility, 30(10), 352-375.

Maurin, E. y McNally, S. (2008). "Vive La Revolution! Long-Term educational returns of 1968 to the angry students", Journal of Labor Economics, 26(1), 1-33.

Mediavilla, M. y Calero, J. (2010). "Movilidad educativa en Latinoamérica. Un estudio para seis países", Revista española de educación comparada, 16, 287-303.

Navarro, A. I. (2010). "Exploring intergenerational educational mobility in Argentina", en Bishop, J. A., ed., Studies in Applied Welfare Analysis: Papers from the Third ECINEQ Meeting (Vol. 18). Bingley: Emerald Group Publishing.

Núñez, J. y Miranda, L. (2011). "Intergenerational income and educational mobility in urban Chile", Estudios de Economía, 38(1), 195-221.

Oreopoulos, P., Page, M. E. y Stevens, A. H. (2006). "The intergenerational effects of compulsory schooling", Journal of Labor Economics, 24(4), 729-760.

Plotno, G. S. (2011). "Movilidad educativa, reclutamiento universitario y educación familiar", Seminario Internacional "Movilidad y cambio social en América Latina", 4 y 5 de noviembre, Mar del Plata, Argentina.

Plug, E. (2004). “Estimating the effect of mother's schooling on children's schooling using a sample of adoptees." American Economic Review, 94(1), 358-68.

Pronzato, C. (2012). “An examination of paternal and maternal intergenerational transmission of schooling." Journal of Population Economics, 25(2), 591-608.

Ranasinghe, R. (2015). "The transmission of education across generations: Evidence from Australia”, The B.E Journal of Economic Analysis and Policy, 15(4), 18931917.

Roemer, J. (1998). Equality of opportunity. Cambridge Mass.: Harvard University Press.

Roemer, J. (2004). "Equal opportunity and intergenerational mobility: going beyond intergenerational income transition matrices", en M. Corak, ed., Generational Income Mobility in North America and Europe. Cambridge: Cambridge University Press.

Rucci, G. (2003). "Macro shocks and schooling decisions: the case of Argentina", Working paper, Departamento de Economía, Universidad de California. 
Sacerdote, B. (2007). "How large are the effects from changes in family environment? A study of Korean American adoptees." Quarterly Journal of Economics, 122(1), $119-57$.

Solon, G. (2004). "A model of intergenerational mobility variation over time and place", en Miles Corak, ed., Generational Income Mobility in North America and Europe. Cambridge: Cambridge University Press.

Stokey, N. L. (1998). "Shirtsleeves to shirtsleeves: the economics of social mobility", en Jacobs, D. P., Kalai, E. y Kamien, M. I., eds., Frontiers of research in economic theory: The Nancy L. Schwartz Memorial Lectures 1983-1997. Cambridge: Cambridge University Press.

Tansel, A. (2011). "Intergenerational educational mobility in Turkey", MPRA paper, 68435.

Torche, F. (2010). "Economic crisis and inequality of educational opportunity in Latin America", Sociology of education, 83(2), 65.110.

Torche, F. (2014). "Intergenerational mobility and inequality: The Latin American case", Annual Review of Sociology, 40, 30.1 -30.24.

Torche, F. (2015). "Intergenerational mobility and equality of opportunity”, European Journal of Sociology, 56(3), 343-371.

Van de Gaer, D., Schokkaert, E. y Martinez, M. (2001), "Three meanings of intergenerational mobility," Economica, 68(272), 519-537. 


\section{Anexo}

\section{Cuadro A1. Coeficiente de persistencia educativa (CPE) y coeficiente de correlación educativa (CCE) por cohortes de nacimiento.}

\begin{tabular}{|c|c|c|c|c|c|c|c|}
\hline \multirow{2}{*}{ Cohorte } & \multicolumn{4}{|c|}{ CPE } & \multicolumn{3}{|c|}{ CCCE } \\
\hline & Modelo 1 & $\mathrm{Moc}$ & lo 2 & Modelo 3 & Modelo 1 & Modelo 2 & Modelo 3 \\
\hline \multicolumn{8}{|l|}{ Varones } \\
\hline \multirow[t]{3}{*}{$1949-58$} & 0.544 & \multirow{2}{*}{\multicolumn{2}{|c|}{$\begin{array}{c}0.633 \\
(0.039)\end{array}$}} & \multirow{2}{*}{$\begin{array}{c}0.542 \\
(0.032)\end{array}$} & 0.524 & \multirow{2}{*}{$\begin{array}{c}0.497 \\
(0.031)\end{array}$} & \multirow{2}{*}{$\begin{array}{c}0.520 \\
(0.031)\end{array}$} \\
\hline & $(0.033)$ & & & & $(0.031)$ & & \\
\hline & $0.480-\quad 0.608$ & $0.556-$ & 0.710 & $0.478-\quad 0.606$ & $0.463-\quad 0.586$ & $0.437-\quad 0.557$ & $0.459-\quad 0.581$ \\
\hline \multirow[t]{3}{*}{ 1959-68 } & \multirow{2}{*}{$\begin{array}{c}0.493 \\
(0.031)\end{array}$} & \multirow{2}{*}{\multicolumn{2}{|c|}{$\begin{array}{c}0.579 \\
(0.035)\end{array}$}} & \multirow{2}{*}{$\begin{array}{c}0.493 \\
(0.031)\end{array}$} & \multirow{2}{*}{$\begin{array}{c}0.498 \\
(0.031)\end{array}$} & \multirow{2}{*}{$\begin{array}{c}0.492 \\
(0.030)\end{array}$} & \multirow{2}{*}{$\begin{array}{c}0.513 \\
(0.024)\end{array}$} \\
\hline & & & & & & & \\
\hline & $0.433-\quad 0.553$ & $0.511-$ & 0.647 & $0.433-\quad 0.553$ & $0.437-\quad 0.558$ & $0.434-\quad 0.550$ & $0.466-\quad 0.560$ \\
\hline \multirow[t]{3}{*}{ 1969-78 } & \multirow{2}{*}{$\begin{array}{c}0.459 \\
(0.026)\end{array}$} & \multirow{2}{*}{\multicolumn{2}{|c|}{$\begin{array}{c}0.489 \\
(0.025)\end{array}$}} & \multirow{2}{*}{$\begin{array}{c}0.457 \\
(0.026)\end{array}$} & \multirow{2}{*}{$\begin{array}{c}0.539 \\
(0.030)\end{array}$} & \multirow{2}{*}{$\begin{array}{c}0.508 \\
(0.026)\end{array}$} & \multirow{2}{*}{$\begin{array}{c}0.523 \\
(0.025)\end{array}$} \\
\hline & & & & & & & \\
\hline & $0.409-\quad 0.509$ & $0.440-$ & 0.539 & $0.407-\quad 0.508$ & $0.480-\quad 0.598$ & $0.457-\quad 0.559$ & $0.475-\quad 0.571$ \\
\hline \multirow[t]{3}{*}{ 1979-88 } & \multirow{2}{*}{$\begin{array}{c}0.404 \\
(0.027)\end{array}$} & & & 0.403 & 0.517 & 0.497 & 0.518 \\
\hline & & & & $(0.027)$ & $(0.035)$ & $(0.035)$ & $(0.025)$ \\
\hline & $0.351-\quad 0.457$ & $0.365-$ & 0.484 & $0.350-\quad 0.456$ & $0.449-\quad 0.585$ & $0.427-\quad 0.566$ & $0.469-\quad 0.567$ \\
\hline Mujeres & & & & & & & \\
\hline $1949-58$ & 0.552 & & & 0.552 & 0.546 & 0.504 & 0.542 \\
\hline & $(0.029)$ & & & $(0.029)$ & $(0.029)$ & $(0.029)$ & $(0.029)$ \\
\hline & $0.495-\quad 0.609$ & $0.553-$ & 0.694 & $0.495-\quad 0.608$ & $0.490-\quad 0.603$ & $0.447-\quad 0.561$ & $0.486-\quad 0.599$ \\
\hline 1959-68 & 0.487 & & & 0.486 & 0.512 & 0.470 & 0.534 \\
\hline & $(0.026)$ & & & $(0.026)$ & $(0.028)$ & $(0.029)$ & $(0.023)$ \\
\hline & $0.435-\quad 0.538$ & $0.486-$ & 0.618 & $0.435-\quad 0.538$ & $0.458-\quad 0.566$ & $0.414-\quad 0.527$ & $0.489-\quad 0.578$ \\
\hline $1969-78$ & 0.439 & & & 0.439 & 0.483 & 0.450 & 0.525 \\
\hline & $(0.024)$ & & & $(0.024)$ & $(0.026)$ & $(0.025)$ & $(0.022)$ \\
\hline & $0.393-\quad 0.485$ & $0.429-$ & 0.533 & $0.392-\quad 0.485$ & $0.432-\quad 0.534$ & $0.401-\quad 0.498$ & $0.481-\quad 0.569$ \\
\hline $1979-88$ & 0.334 & & & 0.334 & 0.443 & 0.413 & 0.517 \\
\hline & $(0.029)$ & & & $(0.029)$ & $(0.038)$ & $(0.036)$ & $(0.024)$ \\
\hline & $0.278-\quad 0.391$ & $0.304-$ & 0.427 & 0.277 - & $0.368-\quad 0.518$ & $0.343-\quad 0.483$ & $0.470-\quad 0.563$ \\
\hline Todos & & & & & & & \\
\hline $1949-58$ & 0.548 & & & 0.547 & 0.536 & 0.501 & 0.531 \\
\hline & $(0.022)$ & & & $(0.022)$ & $(0.021)$ & $(0.021)$ & $(0.021)$ \\
\hline & $0.506-\quad 0.591$ & $0.576-$ & 0.680 & $0.504-\quad 0.589$ & $0.494-\quad 0.577$ & $0.459-\quad 0.542$ & $0.490-\quad 0.572$ \\
\hline 1959-68 & 0.489 & & & 0.490 & 0.505 & 0.481 & 0.524 \\
\hline & $(0.020)$ & & & $(0.020)$ & $(0.021)$ & $(0.021)$ & $(0.016)$ \\
\hline & $0.450-\quad 0.528$ & $0.516-$ & 0.611 & $0.451-\quad 0.529$ & $0.465-\quad 0.546$ & $0.440-\quad 0.521$ & $0.492-\quad 0.556$ \\
\hline 1969-78 & 0.449 & & & 0.447 & 0.510 & 0.478 & 0.524 \\
\hline & $(0.017)$ & & & $(0.017)$ & $(0.020)$ & $(0.018)$ & $(0.017)$ \\
\hline & $0.415-\quad 0.483$ & $0.449-$ & 0.521 & $0.413-\quad 0.481$ & $0.471-\quad 0.549$ & $0.443-\quad 0.513$ & $0.492-\quad 0.557$ \\
\hline $1979-88$ & 0.366 & & & 0.364 & 0.478 & 0.452 & 0.515 \\
\hline & $(0.020)$ & & & $(0.020)$ & $(0.026)$ & $(0.025)$ & $(0.017)$ \\
\hline & $0.327-\quad 0.405$ & $0.351-$ & 0.437 & $0.324-\quad 0.403$ & $0.427-\quad 0.529$ & $0.403-\quad 0.502$ & $0.482-\quad 0.549$ \\
\hline
\end{tabular}

Nota: El modelo 1 tiene como variable dependiente los años de educación de los hijos y variable explicativa los años de educación del padre y una dummy por género (en el modelo estimado para todos los hijos), Modelo 2 es igual al anterior pero utilizando como medida de la educación de padres e hijos su máximo nivel educativo. Estos modelos se estiman para cada cohorte de nacimiento por separado. En cambio el Modelo 3 se estima incluyendo los datos de todas las cohortes de nacimiento y tiene como variable dependiente los años de educación de los hijos y como variables explicativas los años de educación del padre, dummies por cohortes de nacimiento, interacciones entre la educación del padre y las dummies de cohorte y la edad de los hijos. Entre paréntesis se reportan los errores estándares robustos y debajo los intervalos de confianza al 95\%.

Fuente: Elaboración propia sobre la base de Latinobarómetro 2003-2013. 
Cuadro A2. Matrices de transición educativa por cohorte de nacimiento. Varones.

\begin{tabular}{|c|c|c|c|c|c|c|}
\hline \multirow[b]{2}{*}{ Educación padre } & \multicolumn{6}{|c|}{ Educación del hijo } \\
\hline & $\begin{array}{c}\text { Primario } \\
\text { incompleto }\end{array}$ & $\begin{array}{l}\text { Primario } \\
\text { completo }\end{array}$ & $\begin{array}{l}\text { Secundario } \\
\text { incompleto }\end{array}$ & $\begin{array}{c}\text { Secundario } \\
\text { completo }\end{array}$ & $\begin{array}{c}\text { Superior } \\
\text { incompleto }\end{array}$ & $\begin{array}{l}\text { Superior } \\
\text { completo }\end{array}$ \\
\hline \multicolumn{7}{|l|}{ Cohorte 1949-1958 } \\
\hline Primario incompleto & $0.263(0.027)$ & $0.398(0.030)$ & $0.153(0.021)$ & $0.101(0.016)$ & $0.050(0.013)$ & $0.035(0.012)$ \\
\hline Primario completo & $0.045(0.012)$ & $0.378(0.027)$ & $0.234(0.023)$ & $0.196(0.020)$ & $0.060(0.014)$ & $0.087(0.016)$ \\
\hline Secundario incompleto & $0.000(0.000)$ & $0.232(0.102)$ & $0.252(0.108)$ & $0.267(0.099)$ & $0.248(0.107)$ & $0.000(0.000)$ \\
\hline Secundario completo & $0.047(0.027)$ & $0.088(0.035)$ & $0.074(0.032)$ & $0.228(0.047)$ & $0.216(0.053)$ & $0.346(0.059)$ \\
\hline Superior incompleto & $0.000(0.000)$ & $0.116(0.109)$ & $0.109(0.104)$ & $0.104(0.100)$ & $0.110(0.104)$ & $0.560(0.168)$ \\
\hline Superior completo & $.000(0.000)$ & $0.000(0.000)$ & $0.045(0.044)$ & $0.167(0.071)$ & $0.290(0.099)$ & $0.499(0.106)$ \\
\hline \multicolumn{7}{|l|}{ Cohorte 1959-1968 } \\
\hline Primario incompleto & $0.163(0.026)$ & $0.433(0.035)$ & 0. & $0.130(0.021)$ & $0.071(0.019)$ & $0.056(0.016)$ \\
\hline Primario completo & $0.028(0.009)$ & $0.295(0.023)$ & $0.260(0.022)$ & $0.234(0.019)$ & $(0.016)$ & $0.082(0.014)$ \\
\hline Secundario incompleto & $0.000(0.000)$ & $0.055(0.038)$ & $0.273(0.074)$ & $0.232(0.063)$ & $0.297(0.078)$ & $0.143(0.059)$ \\
\hline Secundario completo & $0.000(0.000)$ & $0.042(0.018)$ & $0.117(0.028)$ & $0.235(0.035)$ & $0.253(0.040)$ & $0.352(0.044)$ \\
\hline Superior incompleto & $0.000(0.000)$ & $0.058(0.057)$ & $0.099(0.068)$ & $0.122(0.068)$ & $0.476(0.125)$ & $0.246(0.107)$ \\
\hline Superior completo & $0.000(0.000)$ & $0.035(0.035)$ & $0.041(0.040)$ & $0.176(0.059)$ & $0.333(0.086)$ & $0.414(0.088)$ \\
\hline \multicolumn{7}{|l|}{ Cohorte 1969-1978 } \\
\hline Primario incompleto & $0.116(0.025)$ & $0.411(0.036)$ & $0.216(0.030)$ & $0.138(0.023)$ & $0.074(0.020)$ & $0.044(0.015)$ \\
\hline ipleto & $0.041(0.010)$ & $0.269(0.023)$ & $0.266(0.022)$ & $0.245(0.020)$ & $0.101(0.016)$ & $0.078(0.014)$ \\
\hline Secundario incompleto & $0.039(0.028)$ & $0.049(0.027)$ & $0.260(0.056)$ & $0.290(0.053)$ & $0.230(0.056)$ & $0.133(0.044)$ \\
\hline Secundario completo & $0.006(0.006)$ & $0.054(0.017)$ & $0.147(0.025)$ & $0.248(0.028)$ & $0.283(0.034)$ & $0.262(0.033)$ \\
\hline Superior incompleto & $0.000(0.000)$ & $0.057(0.055)$ & $0.158(0.086)$ & $0.173(0.074)$ & $0.387(0.114)$ & $0.225(0.099)$ \\
\hline Superior completo & $0.006(0.006)$ & $0.013(0.013)$ & $0.042(0.021)$ & $0.193(0.037)$ & $0.364(0.052)$ & $0.383(0.052)$ \\
\hline \multicolumn{7}{|l|}{ Cohorte 1979-1988 } \\
\hline Primario incompleto & $0.076(0.030)$ & $0.368(0.059)$ & $0.190(0.044)$ & $0.220(0.046)$ & $0.145(0.045)$ & $0.000(0.000)$ \\
\hline Primario completo & $0.027(0.012)$ & $0.237(0.031)$ & $0.278(0.030)$ & $0.254(0.028)$ & $0.127(0.025)$ & $0.076(0.019)$ \\
\hline Secundario incompleto & $0.000(0.000)$ & $0.056(0.038)$ & $0.165(0.054)$ & $0.380(0.073)$ & $0.287(0.075)$ & $0.112(0.053)$ \\
\hline Secundario completo & $0.008(0.008)$ & $0.077(0.023)$ & $0.104(0.023)$ & $0.350(0.038)$ & $0.294(0.039)$ & $0.167(0.033)$ \\
\hline Superior incompleto & $0.045(0.045)$ & $0.000(0.000)$ & $0.116(0.107)$ & $0.283(0.126)$ & $0.274(0.134)$ & $0.283(0.137)$ \\
\hline Superior completo & $0.000(0.000)$ & $0.015(0.015)$ & $0.053(0.024)$ & $0.160(0.038)$ & $0.346(0.054)$ & $0.426(0.057)$ \\
\hline
\end{tabular}


Cuadro A3. Matrices de transición educativa por cohorte de nacimiento. Mujeres.

\begin{tabular}{|c|c|c|c|c|c|c|}
\hline \multirow[b]{2}{*}{ Educación padre } & \multicolumn{6}{|c|}{ Educación del hija } \\
\hline & $\begin{array}{c}\text { Primario } \\
\text { incompleto }\end{array}$ & $\begin{array}{l}\text { Primario } \\
\text { completo }\end{array}$ & $\begin{array}{l}\text { Secundario } \\
\text { incompleto }\end{array}$ & $\begin{array}{c}\text { Secundario } \\
\text { completo }\end{array}$ & $\begin{array}{c}\text { Superior } \\
\text { incompleto }\end{array}$ & $\begin{array}{l}\text { Superior } \\
\text { completo }\end{array}$ \\
\hline \multicolumn{7}{|l|}{ Cohorte 1949-1958 } \\
\hline Primario incompleto & $0.293(0.027)$ & $0.415(0.029)$ & $0.124(0.019)$ & $0.087(0.015)$ & $0.039(0.012)$ & $0.042(0.012)$ \\
\hline Primario completo & $0.059(0.012)$ & $0.287(0.024)$ & $0.180(0.020)$ & $0.248(0.021)$ & $0.103(0.017)$ & $0.122(0.018)$ \\
\hline Secundario incompleto & $0.049(0.048)$ & $0.153(0.082)$ & $0.090(0.063)$ & $0.267(0.087)$ & $0.050(0.049)$ & $0.391(0.106)$ \\
\hline Secundario completo & $0.016(0.016)$ & $0.067(0.029)$ & $0.091(0.034)$ & $0.373(0.053)$ & $0.204(0.049)$ & $0.250(0.052)$ \\
\hline Superior incompleto & $0.000(0.000)$ & $0.132(0.123)$ & $0.000(0.000)$ & $0.000(0.000)$ & $0.268(0.163)$ & $0.601(0.178)$ \\
\hline Superior completo & $0.028(0.027)$ & $0.020(0.020)$ & $0.039(0.028)$ & $0.161(0.051)$ & $0.222(0.066)$ & $0.530(0.077)$ \\
\hline \multicolumn{7}{|l|}{ Cohorte 1959-1968 } \\
\hline Primario incompleto & $0.157(0.022)$ & $0.401(0.030)$ & $0.208(0.024)$ & $0.131(0.018)$ & $0.041(0.013)$ & $0.062(0.015)$ \\
\hline Primario completo & $0.032(0.009)$ & $0.276(0.022)$ & $0.204(0.019)$ & $0.241(0.019)$ & $0.085(0.014)$ & $0.161(0.018)$ \\
\hline Secundario incompleto & $0.030(0.029)$ & $0.031(0.030)$ & $0.247(0.072)$ & $0.367(0.075)$ & $0.145(0.062)$ & $0.180(0.067)$ \\
\hline Secundario completo & $0.012(0.012)$ & $0.043(0.021)$ & $0.092(0.030)$ & $0.255(0.040)$ & $0.185(0.040)$ & $0.413(0.050)$ \\
\hline Superior incompleto & $0.024(0.024)$ & $0.041(0.040)$ & $0.000(0.000)$ & $0.121(0.059)$ & $0.363(0.109)$ & $0.451(0.111)$ \\
\hline Superior completo & $0.030(0.029)$ & $0.021(0.021)$ & $0.074(0.032)$ & $0.131(0.041)$ & $0.154(0.051)$ & $0.589(0.068)$ \\
\hline \multicolumn{7}{|l|}{ Cohorte 1969-1978 } \\
\hline Primario incompleto & $0.103(0.022)$ & $0.393(0.033)$ & $0.196(0.027)$ & $0.175(0.023)$ & $0.068(0.018)$ & $0.065(0.017)$ \\
\hline Primario completo & $0.031(0.008)$ & $0.233(0.020)$ & $0.206(0.018)$ & $0.259(0.018)$ & $0.124(0.016)$ & $0.147(0.017)$ \\
\hline Secundario incompleto & $0.019(0.019)$ & $0.185(0.053)$ & $0.120(0.043)$ & $0.255(0.055)$ & $0.183(0.055)$ & $0.238(0.058)$ \\
\hline Secundario completo & $0.006(0.006)$ & $0.042(0.016)$ & $0.132(0.025)$ & $0.249(0.029)$ & $0.178(0.030)$ & $0.392(0.037)$ \\
\hline Superior incompleto & $0.000(0.000)$ & $0.052(0.051)$ & $0.241(0.095)$ & $0.178(0.071)$ & $0.312(0.105)$ & $0.216(0.095)$ \\
\hline Superior completo & $0.007(0.007)$ & $0.000(0.000)$ & $0.059(0.026)$ & $0.153(0.037)$ & $0.219(0.050)$ & $0.563(0.057)$ \\
\hline \multicolumn{7}{|l|}{ Cohorte 1979-1988 } \\
\hline Primario incompleto & $0.139(0.036)$ & $0.213(0.044)$ & $0.256(0.043)$ & $0.206(0.039)$ & $0.090(0.030)$ & $0.095(0.032)$ \\
\hline Primario completo & $0.017(0.009)$ & $0.162(0.025)$ & $0.249(0.027)$ & $0.285(0.027)$ & $0.200(0.028)$ & $0.087(0.019)$ \\
\hline Secundario incompleto & $0.021(0.021)$ & $0.113(0.043)$ & $0.172(0.047)$ & $0.229(0.051)$ & $0.373(0.066)$ & $0.092(0.039)$ \\
\hline Secundario completo & $0.009(0.009)$ & $0.048(0.019)$ & $0.094(0.024)$ & $0.286(0.037)$ & $0.257(0.039)$ & $0.306(0.041)$ \\
\hline Superior incompleto & $0.000(0.000)$ & $0.062(0.060)$ & $0.078(0.054)$ & $0.050(0.049)$ & $0.566(0.118)$ & $0.245(0.105)$ \\
\hline Superior completo & $0.000(0.000)$ & $0.014(0.014)$ & $0.083(0.030)$ & $0.171(0.041)$ & $0.428(0.060)$ & $0.305(0.056)$ \\
\hline
\end{tabular}


Cuadro A4. Índices OV para evaluar QEO por nivel educativo del padre y cohorte de nacimiento. Varones

\begin{tabular}{|c|c|c|c|c|c|c|c|c|}
\hline \multirow{2}{*}{$\begin{array}{l}\text { Cohorte de } \\
\text { nacimiento }\end{array}$} & \multicolumn{7}{|c|}{ Nivel educativo del padre } & \multirow[b]{2}{*}{ Total } \\
\hline & $\begin{array}{c}\text { Primario } \\
\text { incompleto }\end{array}$ & $\begin{array}{l}\text { Primario } \\
\text { completo }\end{array}$ & $\begin{array}{l}\text { Secundario } \\
\text { incompleto }\end{array}$ & $\begin{array}{r}\text { Secundar } \\
\text { complet }\end{array}$ & $\begin{array}{l}\text { Irio } \\
\text { to }\end{array}$ & $\begin{array}{c}\text { Superior } \\
\text { incompleto }\end{array}$ & $\begin{array}{l}\text { Superior } \\
\text { completo }\end{array}$ & \\
\hline \multirow[t]{2}{*}{ [1] 1949-58 } & 0.806 & 0.875 & 0.658 & 0.562 & & 0.521 & 0.397 & 0.537 \\
\hline & $(0.109)$ & $(0.144)$ & $(0.270)$ & $(0.089)$ & & $(0.255)$ & $(0.027)$ & $(0.065)$ \\
\hline \multirow[t]{2}{*}{ [2] 1959-68 } & 0.725 & 0.881 & 0.733 & 0.638 & & 0.554 & 0.528 & 0.569 \\
\hline & $(0.065)$ & $(0.025)$ & $(0.036)$ & $(0.111)$ & & $(0.105)$ & $(0.103)$ & $(0.036)$ \\
\hline \multirow[t]{2}{*}{ [3] 1969-78 } & 0.719 & 0.859 & 0.821 & 0.744 & & 0.704 & 0.570 & 0.632 \\
\hline & $(0.172)$ & $(0.116)$ & $(0.033)$ & $(0.035)$ & & $(0.045)$ & $(0.094)$ & $(0.029)$ \\
\hline \multirow[t]{2}{*}{ [4] 1979-88 } & 0.726 & 0.818 & 0.824 & 0.829 & & 0.778 & 0.597 & 0.686 \\
\hline & $(0.188)$ & $(0.041)$ & $(0.155)$ & $(0.034)$ & & $(0.084)$ & $(0.045)$ & $(0.045)$ \\
\hline \multicolumn{9}{|c|}{ Diferencias entre cohortes } \\
\hline \multirow[t]{2}{*}[4]{$-[1]$} & -0.080 & -0.057 & 0.166 & 0.266 & *** & 0.258 & $0.199 \quad * * *$ & 0.149 \\
\hline & $(0.469)$ & $(0.689)$ & $(0.237)$ & $(0.006)$ & & $(0.188)$ & $(0.000)$ & $(0.159)$ \\
\hline \multirow[t]{2}{*}[4]{$-[3]$} & 0.006 & -0.041 & 0.002 & 0.085 & $*$ & 0.074 & 0.027 & 0.054 \\
\hline & $(0.924)$ & $(0.725)$ & $(0.989)$ & $(0.086)$ & & $(0.541)$ & $(0.828)$ & $(0.343)$ \\
\hline \multirow[t]{2}{*}[3]{$-[2]$} & -0.006 & -0.021 & $0.088 *$ & 0.106 & & $0.150 \quad * *$ & 0.041 & 0.064 \\
\hline & $(0.964)$ & $(0.876)$ & $(0.078)$ & $(0.211)$ & & $(0.050)$ & $(0.455)$ & $(0.272)$ \\
\hline \multirow[t]{2}{*}[2]{$-[1]$} & -0.080 & 0.006 & 0.075 & 0.076 & $* *$ & 0.033 & 0.131 & 0.032 \\
\hline & $(0.223)$ & $(0.973)$ & $(0.770)$ & $(0.016)$ & & $(0.918)$ & $(0.236)$ & $(0.722)$ \\
\hline \multirow[t]{2}{*}{ [4]-[2] } & 0.000 & -0.063 & 0.091 & 0.191 & & 0.224 & 0.069 & $0.117^{* *}$ \\
\hline & $(0.998)$ & $(0.242)$ & $(0.519)$ & $(0.103)$ & & $(0.216)$ & $(0.619)$ & $(0.013)$ \\
\hline \multirow[t]{2}{*}[3]{$-[1]$} & -0.086 & -0.016 & 0.164 & 0.182 & *** & 0.184 & $0.172 *$ & 0.095 \\
\hline & $(0.338)$ & $(0.748)$ & $(0.563)$ & $(0.006)$ & & $(0.523)$ & $(0.074)$ & $(0.117)$ \\
\hline
\end{tabular}

Fuente: Elaboración propia sobre la base de Latinobarómetro 2003-2013.

Cuadro A5. Índices OV para evaluar QEO por nivel educativo del padre y cohorte de nacimiento. Mujeres

\begin{tabular}{|c|c|c|c|c|c|c|c|c|}
\hline \multirow{2}{*}{$\begin{array}{l}\text { Cohorte de } \\
\text { nacimiento }\end{array}$} & \multicolumn{6}{|c|}{ Nivel educativo del padre } & \multirow{2}{*}{\multicolumn{2}{|c|}{ Total }} \\
\hline & $\begin{array}{c}\text { Primario } \\
\text { incompleto }\end{array}$ & $\begin{array}{l}\text { Primario } \\
\text { completo }\end{array}$ & $\begin{array}{c}\text { Secundario } \\
\text { incompleto }\end{array}$ & $\begin{array}{l}\text { Secundario } \\
\text { completo }\end{array}$ & $\begin{array}{c}\text { Superior } \\
\text { incompleto }\end{array}$ & $\begin{array}{l}\text { Superior } \\
\text { completo }\end{array}$ & & \\
\hline [1] 1949-58 & $\begin{array}{c}0.726 \\
(0.060)\end{array}$ & $\begin{array}{c}0.896 \\
(0.093)\end{array}$ & $\begin{array}{c}0.674 \\
(0.134)\end{array}$ & $\begin{array}{c}0.600 \\
(0.291)\end{array}$ & $\begin{array}{c}0.364 \\
(0.314)\end{array}$ & $\begin{array}{c}0.481 \\
(0.103)\end{array}$ & $\begin{array}{c}0.530 \\
(0.039)\end{array}$ & \\
\hline [2] 1959-68 & $\begin{array}{c}0.742 \\
(0.026)\end{array}$ & $\begin{array}{c}0.925 \\
(0.022)\end{array}$ & $\begin{array}{c}0.724 \\
(0.037)\end{array}$ & $\begin{array}{c}0.639 \\
(0.088)\end{array}$ & $\begin{array}{c}0.472 \\
(0.049)\end{array}$ & $\begin{array}{c}0.543 \\
(0.163)\end{array}$ & $\begin{array}{c}0.574 \\
(0.048)\end{array}$ & \\
\hline [3] 1969-78 & $\begin{array}{c}0.733 \\
(0.184)\end{array}$ & $\begin{array}{c}0.917 \\
(0.159)\end{array}$ & $\begin{array}{c}0.900 \\
(0.092)\end{array}$ & $\begin{array}{c}0.757 \\
(0.054)\end{array}$ & $\begin{array}{c}0.753 \\
(0.302)\end{array}$ & $\begin{array}{c}0.565 \\
(0.044)\end{array}$ & $\begin{array}{c}0.622 \\
(0.060)\end{array}$ & \\
\hline [4] 1979-88 & $\begin{array}{c}0.727 \\
(0.278) \\
\end{array}$ & $\begin{array}{c}0.855 \\
(0.102) \\
\end{array}$ & $\begin{array}{c}0.877 \\
(0.347) \\
\end{array}$ & $\begin{array}{c}0.815 \\
(0.039) \\
\end{array}$ & $\begin{array}{c}0.607 \\
(0.229) \\
\end{array}$ & $\begin{array}{c}0.685 \\
(0.174) \\
\end{array}$ & $\begin{array}{c}0.723 \\
(0.111) \\
\end{array}$ & \\
\hline \multicolumn{9}{|c|}{ Diferencias entre cohortes } \\
\hline$[4]-[1]$ & $\begin{array}{c}0.001 \\
(0.997) \\
\end{array}$ & $\begin{array}{l}-0.041 \\
(0.505) \\
\end{array}$ & $\begin{array}{c}0.203 \\
(0.652) \\
\end{array}$ & $\begin{array}{c}0.215 \\
(0.465) \\
\end{array}$ & $\begin{array}{c}0.242 \\
(0.286) \\
\end{array}$ & $\begin{array}{c}0.204 \\
(0.417) \\
\end{array}$ & $\begin{array}{c}0.193 \\
(0.029) \\
\end{array}$ & $* *$ \\
\hline$[4]-[3]$ & $\begin{array}{l}-0.006 \\
(0.989) \\
\end{array}$ & $\begin{array}{l}-0.062 \\
(0.512) \\
\end{array}$ & $\begin{array}{c}-0.023 \\
(0.954) \\
\end{array}$ & $\begin{array}{c}0.058 \\
(0.381) \\
\end{array}$ & $\begin{array}{l}-0.146 \\
(0.553) \\
\end{array}$ & $\begin{array}{c}0.120 \\
(0.560) \\
\end{array}$ & $\begin{array}{c}0.102 \\
(0.134) \\
\end{array}$ & \\
\hline$[3]-[2]$ & $\begin{array}{c}-0.009 \\
(0.957) \\
\end{array}$ & $\begin{array}{l}-0.008 \\
(0.962) \\
\end{array}$ & $\begin{array}{cc}0.175 & * * \\
(0.029) & \\
\end{array}$ & $\begin{array}{cc}0.118 & * * \\
(0.023) & \\
\end{array}$ & $\begin{array}{c}0.281 \\
(0.302) \\
\end{array}$ & $\begin{array}{c}0.023 \\
(0.905) \\
\end{array}$ & $\begin{array}{c}0.048 \\
(0.646) \\
\end{array}$ & \\
\hline$[2]-[1]$ & $\begin{array}{c}0.016 \\
(0.788) \\
\end{array}$ & $\begin{array}{c}0.029 \\
(0.767) \\
\end{array}$ & $\begin{array}{c}0.051 \\
(0.675) \\
\end{array}$ & $\begin{array}{c}0.038 \\
(0.914) \\
\end{array}$ & $\begin{array}{c}0.108 \\
(0.704) \\
\end{array}$ & $\begin{array}{c}0.061 \\
(0.784) \\
\end{array}$ & $\begin{array}{c}0.044 \\
(0.586) \\
\end{array}$ & \\
\hline$[4]-[2]$ & $\begin{array}{l}-0.015 \\
(0.959) \\
\end{array}$ & $\begin{array}{l}-0.070 \\
(0.528) \\
\end{array}$ & $\begin{array}{c}0.152 \\
(0.672) \\
\end{array}$ & $\begin{array}{cc}0.177 & * \\
(0.053) & \\
\end{array}$ & $\begin{array}{c}0.134 \\
(0.503) \\
\end{array}$ & $\begin{array}{c}0.143 \\
(0.412) \\
\end{array}$ & $\begin{array}{c}0.150 \\
(0.331) \\
\end{array}$ & \\
\hline [3]-[1] & $\begin{array}{c}0.007 \\
(0.969) \\
\end{array}$ & $\begin{array}{c}0.021 \\
(0.793) \\
\end{array}$ & $\begin{array}{cc}0.226 & * * * \\
(0.001) & \\
\end{array}$ & $\begin{array}{c}0.157 \\
(0.630) \\
\end{array}$ & $\begin{array}{cc}0.389 & * * * \\
(0.007) & \\
\end{array}$ & $\begin{array}{c}0.084 \\
(0.357) \\
\end{array}$ & $\begin{array}{c}0.092 \\
(0.055) \\
\end{array}$ & \\
\hline
\end{tabular}

Fuente: Elaboración propia sobre la base de Latinobarómetro 2003-2013. 


\section{Capítulo 2*}

\section{Movilidad intergeneracional del ingreso en Argentina: evaluando métodos y comparando resultados con Chile}

\section{Introducción}

Dado el objetivo general de la tesis, en este capítulo se estima y analiza el nivel de movilidad socioeconómica intergeneracional en Argentina. Pero, mientras que en el capítulo anterior se utilizó la educación como medida del nivel socioeconómico de padres e hijos, en este caso, se considera el ingreso.

Los motivos para examinar la transmisión del ingreso entre generaciones son varios. Así, el análisis de la MII complementa las investigaciones sobre la desigualdad del ingreso que estudian la distribución desde un punto de vista estático. Por otra parte, comprender el grado de movilidad generacional es un primer paso para entender las consecuencias de la desigualdad de ingresos (Cruces, 2008). En esta línea, Kuznets (1966) señala que el significado de dos sociedades con idénticas distribuciones de ingresos puede diferir ampliamente según los grados de movilidad interna. Rosen (1985) va más allá al afirmar que si existe suficiente movilidad del ingreso no es necesario preocuparse de cuán desigualmente están distribuidos los ingresos.

A pesar de los diversos motivos que justifican el estudio de la MII, la obtención de una medida apropiada del grado de asociación que existe entre el ingreso de padres e hijos es difícil de obtener. Esto resulta particularmente problemático en países que, como Argentina, no cuentan con fuentes de datos apropiadas para ello. Por esto, el primer objetivo de este capítulo es evaluar los distintos métodos existentes para estimar

\footnotetext{
- Agradezco las observaciones de Leonardo Gasparini y Jorge Paz así como los comentarios realizados por el Comité de Doctorado, por los asistentes al Seminario de Doctorado de la UNLP y los recibidos durante la XLVII Reunión Anual de la AAEP.
} 
la elasticidad intergeneracional del ingreso (EII) que constituye la medida de inmovilidad de ingresos entre generaciones más utilizada en la literatura. Con este fin, se analizan los límites en probabilidad los estimadores de la EII disponibles en la literatura, en el contexto del modelo intergeneracional tradicional del ingreso permanente. Este análisis permite discutir formalmente los potenciales sesgos presentes en estos estimadores así como los supuestos necesarios para determinar su consistencia. Asimismo, se obtienen y examinan empíricamente la mayoría de ellos. Para esto resultan particularmente útiles los datos de la Encuesta Panel CASEN 1996-2001-2006 (EPCASEN) de Chile que constituye uno de los paneles de datos más largo disponible para un país de América Latina.

El segundo objetivo de esta investigación es estimar y analizar los niveles así como los cambios temporales en la MII en Argentina durante el período más extenso posible. Asimismo, a fin de valorar la magnitud del grado promedio de persistencia de ingresos entre generaciones observado en el país, se realiza también un análisis comparativo con Chile. Además de los motivos metodológicos, esta comparación es, en sí misma, un ejercicio interesante por diversas razones. En primer lugar, la obtención de estimaciones metodológicamente comparables permite evaluar si los niveles de MII en Argentina pueden considerarse relativamente altos o bajos en relación con los computados para Chile, evaluando así el desempeño relativo del país en esta dimensión dinámica del bienestar. Esto, a su vez, permite eliminar el efecto de distintas muestras de padres e hijos y métodos en las diferencias que pueden observarse. Por otra parte, las características comunes y disímiles que presentan estos dos países limítrofes y que, según los modelos teóricos, son susceptibles de tener un efecto importante sobre el proceso de transmisión intergeneracional, podrían permitir elaborar futuras hipótesis susceptibles de explicar las potenciales diferencias o similitudes observadas en los niveles de MII entre ambos países. Así, por ejemplo, aunque ambos países comparten políticas sociales universalistas, los patrones de crecimiento económico que experimentaron no han sido convergentes. Además, conforme con la evidencia reportada por Gasparini et al. (2007), para la década del noventa y principios de los 2000s, mientras en Argentina los cambios en el ingreso han sido negativos y claramente desigualadores, Chile ha experimentado un crecimiento sostenido a lo largo de toda la distribución de ingresos. La evolución de la incidencia de la pobreza también muestra marcadas heterogeneidades entre Chile y Argentina, durante los 90s y principios de los 
2000s. Así, mientras en Chile se observa reducciones significativas (al igual que en Brasil, El Salvador, Jamaica y Nicaragua), Argentina muestra un gran incremento de la tasa de pobreza en ese período. Por otra parte, ambos países han experimentado significativos cambios políticos y económicos en las últimas décadas. Después de una profunda recesión a principios de los 80 , la economía chilena exhibió un substancial y sostenido crecimiento económico durante los 90s que llevó a una mejora significativa de las condiciones de vida en el país. El lado oscuro de esta exitosa historia es la persistente desigualdad económica que ha sido históricamente elevada. Otra característica que comparte con la economía argentina, en la que, en cambio, el crecimiento económico no fue tan elevado ni sostenido como el chileno ${ }^{49}$. El patrón de desigualdad chileno está caracterizado por una alta concentración de los ingresos entre los estratos más ricos de la población, mientras que las diferencias entre los sectores pobres y aquellos de ingresos medios son mucho menos pronunciadas que las observadas en naciones industrializadas (Torche, 2005).

A partir de los 2000s, concretamente, entre 2002 y 2008, la desigualdad comenzó a disminuir en casi todas las economías de América Latina, incluyendo Chile y Argentina, después de la tendencia creciente observada durante la denominada "década perdida" de los $80 \mathrm{~s}$, la reformas estructurales de los $90 \mathrm{~s}$ y las crisis ocurridas al cambiar el siglo (Gasparini y Lustig, 2011). No obstante, tanto en los 90s como en los 2000s, los niveles de desigualdad del ingreso en Chile fueron mayores que los observados en Argentina $^{50}$. Si la desigualdad y la movilidad están relacionadas, siendo la primera un factor muy importante para la configuración de la segunda, más que, por ejemplo, los niveles de crecimiento económico, se espera que Chile muestre niveles de persistencia intergeneracional mayores o relativamente similares a Argentina.

Por último, este capítulo también tiene como objetivo examinar empíricamente un modelo intergeneracional alternativo, recientemente propuesto por Muller (2010) y

\footnotetext{
${ }^{49}$ En Argentina, la desigualdad aumentó significativamente desde mediados de la década del setenta hasta mediados de los años 2000. Para un análisis detallado de las tendencias de la desigualdad en Argentina durante ese período así como de las principales hipótesis acerca de los determinantes de esa evolución, ver Gasparini y Cruces (2008). En tanto que, en el estudio de Gasparini y Lustig (2011) puede encontrarse un examen detenido de la evolución temporal de la desigualdad de ingresos en América Latina.

${ }^{50}$ Así, por ejemplo, en 1996 y 2006, dos años particularmente relevantes en esta investigación, el coeficiente de Gini del ingreso familiar per cápita fue de 0.486 y 0.475 , en Argentina, mientras que, en Chile, ascendió a 0.548 y 0.518 , respectivamente, siendo las diferencias entre los coeficientes de ambos países, para los mismos años, estadísticamente significativas.
} 
poco explorado en la literatura. La idea central de este enfoque es que, a diferencia de lo sostenido por el modelo intergeneracional tradicional, la transmisión relevante entre generaciones, no sólo tiene lugar en relación al componente permanente del ingreso sino también, a través de los ingresos transitorios ${ }^{51}$. Esto, a su vez, tiene varias implicancias relevantes. Por un lado, sugiere que las comparaciones realizadas entre países en base al modelo estándar de transmisión intergeneracional del ingreso permanente, ignora una fuente potencial de heterogeneidad inobservada que podría afectar las estimaciones de la EII: la edad de los hijos en la que se mide el ingreso de sus padres. Por otra parte, si es posible identificar que los componentes transitorios del ingreso de los padres tienen un efecto significativo en las oportunidades económicas futuras de los niños, las implicancias de política serían más inmediatas. Si el ingreso transitorio afecta el ingreso futuro de los niños, esto indicaría que el ingreso de los padres per se tiene un efecto en sus oportunidades, lo que generalmente es incompatible con las formulaciones incluso más débiles del enfoque de igualdad de oportunidades. Entonces, cuanto mayor sea la importancia del ingreso transitorio con relación al permanente en el proceso de transmisión intergeneracional, mayor será el campo para la intervención pública (Muller, 2008).

La estimación de este modelo alternativo, al igual que el tradicional, es particularmente problemática en países en los que las bases de datos disponibles no incluyen información sobre el ingreso de los padres para los individuos encuestados, en edades centrales del ciclo vital de ambos. Aunque contienen datos sobre otras características de los padres como su educación. Si bien, una solución ampliamente utilizada para estimar la EII en este caso es implementar el método Two-Sample Intrumental Variable (TSIV), esta técnica no sería adecuada para el modelo alternativo porque instrumenta el ingreso permanente pero no resuelve el problema de la exclusión del ingreso transitorio del modelo estimado. Por eso, en esta investigación se propone una nuevo enfoque metodológico que combina algunas características del método TSIV, para estimar el componente permanente del ingreso parental y del enfoque propuesto

\footnotetext{
${ }^{51}$ Como señala Muller (2010), a quienes no están demasiado familiarizados con la literatura específica de movilidad intergeneracional del ingreso les podría resultar sorprendente descubrir que la posibilidad de que el ingreso parental de cualquier tipo (permanente o transitorio) tenga un efecto causal en las oportunidades futuras de los hijos no sea ampliamente aceptada.
} 
por Dang et al. (2014) ${ }^{52}$ que permite considerar también su componente transitorio, particularmente durante la niñez y adolescencia de los hijos. Bajo determinados supuestos, con esta técnica podría obtenerse un límite inferior y superior de la EII en el contexto del modelo alternativo.

Este capítulo está organizado de la siguiente manera. En la próxima sección se mencionan los principales antecedentes de esta investigación así como su contribución a la literatura existente. A continuación, se analizan los modelos empíricos y los métodos de estimación existentes, describiendo formalmente los potenciales sesgos que pueden presentar, con el objetivo de ofrecer una exposición completa y ordenada de los principales problemas de estimación de los niveles de persistencia intergeneracional del ingreso planteados en distintos estudios y que deben ser tenidos en cuenta al momento de realizar comparaciones entre países. También, se destacan aquí las limitaciones de algunos análisis metodológicos realizados en la literatura, se plantea un modelo intergeneracional alternativo y se propone un nuevo método de estimación. La próxima sección contiene una descripción de las fuentes de datos utilizadas. A continuación se presentan y analizan los resultados obtenidos. Luego, en la última sección, se enuncian las principales conclusiones del capítulo.

\section{La movilidad intergeneracional del ingreso: una revisión de los antecedentes}

\subsection{La medición de la MII en los estudios empíricos}

La mayoría de los estudios empíricos que cuantifican y analizan la transmisión intergeneracional del ingreso en un cierto país o región están referidos a países desarrollados, principalmente por la mayor disponibilidad de datos para esos países. Entre estos, se destacan como antecedentes el artículo por Atkinson et al. (1983) para Inglaterra, así como los trabajos de Solon (1992) y Zimmerman (1992) para Estados

\footnotetext{
${ }^{52}$ Los autores proponen este enfoque para analizar los movimientos intrageneracionales dentro y fuera de la pobreza.
} 
Unidos. Sin embargo, en los últimos años esta literatura ha crecido y se ha extendido en diferentes lugares ${ }^{53}$.

En general, la movilidad del ingreso entre padres e hijos es medida a partir de la EII. La mayoría de los estudios computan la EII a partir de la estimación por Ordinary Least Squares (OLS), de una regresión en la que el logaritmo del ingreso de los hijos es la variable dependiente y el logaritmo del ingreso del padre, la variable explicativa, con controles por edad para ambas generaciones. El método de OLS ha sido ampliamente utilizado para estimar la EII en diferentes años, por numerosos autores y para diversos países $^{54}$.

Como señala Solon (2002), el interés principal en un análisis de la MII es el grado de asociación entre los ingresos de largo plazo de padres e hijos. Sin embargo, por limitaciones informativas, algunos estudios emplean medidas de un único año para los ingresos de los padres. Aunque, los ingresos anuales no reflejan correctamente los ingresos permanentes. Por esta razón, para corregir el sesgo por error de medición en esta variable, algunos autores utilizaron un promedio de los ingresos a lo largo de varios años, lo que reduce el impacto de las variaciones transitorias (Behrman y Taubman, 1985; Solon, 1992; Couch y Dunn, 1997; Blanden et al., 2005). Otro enfoque implementado para solucionar este problema de sesgo fue el método de variables instrumentales ${ }^{55}$.

Al no disponer de información del ingreso de padres e hijos en momentos similares de su ciclo de vida, algunos estudios empíricos recurrieron a la solución propuesta por Arellano y Meghir (1992) así como por Angrist y Krueger (1992) que consiste en utilizar información de dos muestras separadas a fin de predecir el ingreso de los padres cuando los hijos eran niños o adolescentes y obtener una aproximación de

\footnotetext{
${ }^{53}$ Para una revisión más exhaustiva de los estudios existentes sobre MII a nivel internacional, ver Jiménez (2011a).

54 Así, Atkinson et al. (1983), Behrman y Taubman (1985), Solon (1992), Zimmerman (1992), Peters (1992) y Dahl y DeLeire (2008) aplican esa metodología para Estados Unidos; Dearden et al. (1997) y Blanden et al. (2002) lo hacen para Gran Bretaña; Osterberg (2000) y Hirvonen (2008), para Suecia; Österbacka (2001), para Finlandia; Bratberg et al. (2005), para Noruega; Couch y Dunn (1997), para Alemania; Comi (2004), para los países de la Comunidad Europea y Estados Unidos; Blanden et al. (2005), para Gran Bretaña, el Oeste de Alemania, Canadá y Estados Unidos; Corak y Heisz (1999), para Canadá; Sánchez Hugalde (2004), para España, Hertz (2001), para Sudáfrica y Núñez y Risco (2004), para Chile.

${ }_{55}$ Así, en uno de los principales estudios pioneros, Solon (1992) propone utilizar como instrumento del status socioeconómico del padre, sus años de educación.
} 
sus ingresos permanentes. Por esta razón, suele conocerse a este método como TwoSample Two-Stage Least Squares (TS2SLS). Esta técnica de estimación fue implementada mayormente en estudios para países europeos y en desarrollo ${ }^{56}$.

Por otra parte, la mayoría de los estudios existentes sobre MII ofrecen estimaciones de una asociación intergeneracional promedio. Sin embargo, las estimaciones promedio son de poca utilidad para medir la persistencia para aquellos que crecieron en familias de bajos o altos ingresos. Los estudios que analizan la variación del grado de persistencia a lo largo de la distribución emplean, generalmente, el método de regresiones por cuantiles $(\mathrm{QR})^{57}$ y matrices de transición ${ }^{58}$. Los resultados obtenidos por estos estudios sugieren que las probabilidades de alcanzar diferentes cuantiles de ingresos de destino dependen significativamente del cuantil de origen definido en términos de los ingresos de los padres. También muestran que la correlación intergeneracional varía con el cuantil de la distribución del ingreso al que pertenezca el individuo y su padre.

A partir de la revisión de la literatura se advierte una desproporcionada cantidad de estudios realizados para países desarrollados en comparación con los existentes para países en desarrollo. En particular, los estudios sobre MII en Argentina, como se mencionó en la Introducción, son prácticamente inexistentes. Si bien existen estudios de movilidad intergeneracional específicos para el país, la mayoría no examina la movilidad del ingreso sino la movilidad ocupacional o educativa entre generaciones ${ }^{59}$. Beccaria (1978) es uno de los primeros que lo hace, empleando la encuesta de movilidad social organizada, para el Gran Buenos Aires (GBA), como un complemento de la Encuesta de empleo y desempleo de octubre de 1969. A partir de estos datos,

\footnotetext{
${ }^{56}$ Entre los primeros, se encuentran los realizados para Suecia por Björklund y Jänti (1997), para Alemania por Couch y Dunn (1997) y Vogel (2006), para Gran Bretaña por Nicoletti y Ermisch (2008), para Francia por Lefranc y Trannoy (2005), para Australia por Leigh (2007) y para Japón por Lefranc et al. (2010). La mayoría de los escasos estudios de MII para América Latina aplican esta método. Entre ellos se encuentran los trabajos de Grawe (2004a) para Ecuador y Perú, los desarrollados por Núñez y Risco (2004) y Núñez y Miranda (2007) para Chile así como los de Ferreira y Veloso (2006) y Dunn (2007) para Brasil.

${ }^{57}$ Los trabajos empíricos de Eide y Showalter (1999), Grawe (2004a y 2004b) y Brastberg et al. (2007) son algunos de los no tan numerosos estudios que implementan esta metodología.

${ }^{58}$ Entre los diversos estudios que computan matrices de transición se encuentran algunos relativamente recientes como los de Dahl y DeLeire (2008), Núñez y Miranda (2007), Jäntti et al. (2006), Ferreira y Veloso (2006) y otros más antiguos como los de Atkinson et al. (1983), Zimmerman (1992), Peters (1992), entre otros.

${ }^{59}$ En el Capítulo 1 puede consultarse la revisión de esta literatura para el país.
} 
construye matrices de transición que relacionan los estratos sociales de padres e hijos. También, obtiene, entre otros, un "índice bruto de inmovilidad" que mide la proporción de individuos ubicados en el mismo estrato de sus padres y que, para la muestra en su conjunto, tiene un valor de $24 \%$.

Los escasos estudios sobre movilidad ocupacional intergeneracional en Argentina se realizaron en base a datos del GBA (Germani, 1963, Beccaria, 1978, Jorrat, 2000, Chávez Molina y Gutiérrez Ageitos, 2009), salvo el trabajo de Jorrat (2005) que fue desarrollado a partir de dos encuestas nacionales relevadas en $2003 \mathrm{y}$ 2004 por el Centro de Estudios de Opinión Pública (CEDOP) de la Universidad Nacional de Buenos Aires (UBA) y el estudio de Quartulli y Salvia (2012) que utilizaron tres encuestas nacionales urbanas realizadas por el Observatorio de la Deuda social Argentina de la Universidad Católica Argentina (UCA) en 2007, 2008 y 2009. Los resultados obtenidos por Jorrat (2005), a partir del análisis descriptivo muestran una pauta de movilidad ocupacional entre generaciones atendible puesto que el $64,1 \%$ de los encuestados exhibió movilidad de algún tipo respecto de la clase del padre y, además, una prevalencia de movilidad ascendente. Según el autor, estos hallazgos ratificarían la idea de que la vinculación entre crecimiento de la desigualdad y la baja movilidad social no es concluyente.

El antecedente más directo de la investigación desarrollada en este capítulo es el estudio de FIEL (2008) que examina los efectos de un indicador del nivel socioeconómico de los padres en el ingreso de los hijos pero a partir de una encuesta específica del 2007 para el Gran Buenos Aires. Según los resultados obtenidos por los autores, la movilidad intergeneracional de la condición socioeconómica ${ }^{60}$ puede considerarse moderada con respecto a los países más avanzados pero mayor que la observada en Brasil y Colombia aunque con un desempeño similar al de México y Brasil, en la región latinoamericana. El estudio también ofrece un análisis de la movilidad socioeconómica intergeneracional por cohortes, que muestra un decrecimiento del efecto positivo del nivel socioeconómico de los padres sobre el de los hijos a medida que la cohorte envejece. Según los autores, este resultado sugiere que

\footnotetext{
${ }^{60}$ La movilidad intergeneracional socioeconómica es medida a través de la elasticidad intergeneracional y del coeficiente de correlación intergeneracional de ingresos. Sin embargo, para aproximar la medida del ingreso permanente se construyó un índice de nivel socioeconómico que sintetiza la situación patrimonial, educativa y de empleo para cada uno de los encuestados.
} 
Argentina ha perdido movilidad social a lo largo del tiempo aunque la falta de datos longitudinales impiden la obtención de una conclusión más precisa sobre este punto.

En el caso de Chile, en cambio aunque escasos, existen estudios que examinaron la MII ${ }^{61}$ como los de Núñez y Risco (2004) y Núñez y Miranda (2007, 2011) y el más reciente de Celhay et al. (2010). Con excepción de éste último, los anteriores utilizaron datos cross-section, implementando la estrategia de estimación con dos muestras en dos etapas (TSIV) a partir de los microdatos provenientes de Encuesta de Empleo y Desempleo. En cambio, en esta investigación se explotará el panel más largo disponible para Chile: la Encuesta Panel Casen 1996-2001-2006. Además, a diferencia del estudio de Celhay et al. (2010) que también utiliza esta base de datos, los objetivos de esta investigación son distintos. En primer lugar, se pretende obtener estimaciones de los niveles de MII comparables en Chile y Argentina y, en segundo lugar, evaluar la performance de los métodos de estimación basados en datos crosssection como la técnica TSIV, así como un nuevo método aquí propuesto para examinar un modelo intergeneracional alternativo.

\subsection{La evidencia empírica sobre los cambios temporales en la MII}

La mayoría de los estudios que examina las tendencias en la MII lo hacen para Estados Unidos. Entre estos se encuentra el desarrollado por Fertig (2001) que, a partir de los datos del Panel Study of Income Dynamics (PSID), observa cinco cohortes nacidas entre 1968 y 1993 para quienes estima regresiones por OLS, regresiones por cuantiles $(\mathrm{QR})$ y matrices de transición. Los resultados indican que la EII decreció para los hijos y padres en comparación con la computada para las hijas y madres. Además, la diferencia de movilidad entre las cohortes de padres e hijos más ricas y las más pobres parece haberse reducido a lo largo del período bajo estudio.

Mayer y Lopoo $(2004,2005)$ examinan la EII para las cohortes nacidas entre 1949 y 1965. A partir de los datos longitudinales obtenidos del Panel Study of Income Dynamics (PSID) encuentran una tendencia creciente en la movilidad económica

\footnotetext{
${ }^{61}$ Al igual que para Argentina, son más numerosas las investigaciones que examinan la movilidad educativa u ocupacional intergeneracional, como la de Torche (2005) o la más reciente de Sapelli (2011).
} 
intergeneracional. En el más reciente de estos estudios, Mayer y Lopoo (2005) estiman, a partir de una regresión bivariada que relaciona el status económico del padre y del hijo, la EII en el año en que cada cohorte alcanza los 30 años de edad. Los principales resultados indican que la correlación entre el ingreso de los padres y el de los hijos nacidos entre 1963 y 1965 es menor que la estimada entre aquellos nacidos entre 1949 y 1952. Para los autores, esta caída se debe al incremento de la inversión del gobierno en educación.

Por su parte, Lee y Solon (2009) argumentan que haciendo un uso más eficiente del PSID es posible obtener evidencia más confiable de las variaciones recientes en la MII en Estados Unidos. Comparado con estudios previos, el enfoque de Lee y Solon (2009) mejora la precisión de las estimaciones de la EII a lo largo del tiempo al usar los datos disponibles para todas las edades. Las estimaciones de la EII por OLS para hijos e hijas no revelan cambios importantes en la MII entre 1977 y 2000, para las cohortes nacidas entre 1952 y 1975 . Este resultado no es consistente con los obtenidos por los estudios previos, a partir de los datos del PSID, que sugieren un incremento en la MII en Estados Unidos. No obstante, Lee y Solon (2009) reconocen que sus resultados no son los suficientemente precisos como para descartar tendencias modestas en cualquier dirección.

Otro de los estudios que examina las tendencias en la MII en Estados Unidos es el desarrollado por Hertz (2007) utilizando también los datos del PSID. El autor analiza las cohortes nacidas entre 1952 y 1975 durante el período 1977- 2000. A partir de la estimación de regresiones por OLS, Hertz (2007) encuentra que no existe una tendencia lineal en la elasticidad del ingreso familiar ni en la del ingreso familiar per capita entre 1977 y 2000. Según Hertz (2007) los resultados de la estimaciones de la trayectoria temporal de la EII pueden estar afectados por la forma en que las tendencias son definidas (en cohortes o en años), por la edad en la que cada cohorte es observada así como por el método empleado para corregir el problema de desgranamiento y de selección muestral. La evidencia encontrada por Hertz (2007) es consistente con los resultados obtenidos por Lee y Solon (2009) y por Harding et al. (2004) que emplean una muestra obtenida también de los datos del PSID, de las denominadas Occupational Changes in a Generation Surveys y General Social Survey para EE.UU. 
Entre los estudios más recientes que examinan las tendencias en la MII en Estados Unidos se encuentra el desarrollado por Aaronson y Mazumder (2008). A partir de los datos del censo de Estados Unidos, los autores estiman una serie de tiempo de la MII utilizando el método de variables instrumentales en dos muestras (TSIV). La estrategia de estimación implementada consiste en calcular el ingreso familiar promedio por estado de nacimiento para cada cohorte considerada. Las tendencias encontradas en la MII muestran una forma de U invertida entre 1940 y 2000. Para Aaronson y Mazumder (2008), ese patrón es similar a las tendencias observadas en la desigualdad del ingreso dado que los factores subyacentes que determinan las trayectorias de ambas variables son los mismos.

Entre los relativamente pocos estudios que examinan las tendencias en la MII para países europeos pueden mencionarse el de Bratberg et al. (2005) para Noruega, Pekkala y Lucas (2007), para Finlandia y el realizado por Lefranc (2010), para Francia. A partir de los datos de la Norwegian Database of Generations (DBG), Bratberg et al. (2005) examinan las tendencias en la MII en Noruega para cohortes nacidas en 1950, 1955 y 1960. Utilizando regresiones por cuantiles, los autores comparan los patrones de movilidad del ingreso en diferentes puntos de la distribución del ingreso de los hijos. Los resultados sugieren que la EII es relativamente baja en el país y que la movilidad parece haber crecido para la mayoría de las cohortes más recientes. Además, aunque la EII es mayor para los niños de los quintiles más pobres en relación a los que pertenecen al extremo superior de la distribución del ingreso, entre los primeros, la movilidad creció durante el período. Al mismo tiempo, los resultados indican que la reducción de la EII en el tiempo es mayor para las mujeres que para los varones. En general, Bratberg et al. (2005) sostienen que gran parte de la evidencia encontrada es resultado de la implementación de políticas tendientes a igualar las oportunidades entre individuos, sobre todo aquellas relacionadas con las reformas en escuelas y el acceso al crédito.

Con el objetivo de analizar las tendencias de la MII familiar en Finlandia, Pekkala y Lucas (2007) emplean datos de panel obtenidos de la Central Population Register, Tax Register y Longitudinal Employment Statistics de 1950 a 1999. Las EII estimadas por OLS indican que la movilidad se incrementó para la cohortes nacidas después de 1930 hasta 1950 pero disminuyó para las nacidas entre este último año y 1960, aunque no significativamente. Según los autores, la movilidad más baja que 
experimentan las generaciones nacidas durante 1930 y la segunda guerra mundial coincide con un período en el que la desigualdad de ingreso fue relativamente alta en Finlandia, donde la escolaridad secundaria estaba reservada a las grandes elites de la sociedad y la mayoría de la población se dedicaba a la agricultura. Es por ello que, para Pekkala y Lucas (2007), el mayor acceso a las escuelas secundarias, especialmente en las áreas rurales es el factor clave determinante del incremento de la movilidad intergeneracional de las cohortes nacidas después de 1930. No obstante, las estimaciones indican que no existen reducciones significativas en la EII para las cohortes más recientes a pesar del mayor acceso a la educación terciaria que existió durante ese período.

En un estudio reciente, Lefranc (2010) utilizan datos obtenidos de las encuestas de Formation, Qualification, Profession (FPQ) desde 1964 a 2003 para estimar las tendencias en la MII en Francia utilizando el método de TSIV. El autor encuentra un patrón en forma de U invertida en la EII para las cohortes de varones nacidos entre 1933 y 1975. En particular, los resultados sugieren un decrecimiento de la MII para aquellos nacidos entre la mitad de la década del 30 y 1950 pero un posterior incremento para las cohortes siguientes que puede explicarse, según Lefranc (2010), por una menor asociación entre la educación de los padres e hijos así como por una disminución de los retornos a la educación.

En conclusión, aunque son varias las investigaciones que analizan las tendencias en la movilidad intergeneracional, la mayoría fueron desarrolladas para Estados Unidos. No obstante, muy pocas adoptaron un enfoque metodológico, como el implementado en este estudio, que permita obtener estimaciones de la EII a lo largo del tiempo más precisas que las computadas considerando los datos disponibles de cohortes determinadas para cada período. Por otra parte, los estudios disponibles que analizan la evolución temporal de la movilidad intergeneracional para otros países desarrollados son bastante menos numerosos que los encontrados para Estados Unidos y resultan muy escasos los realizados para países en desarrollo. En particular, los estudios que examinan los cambios en la MII en el tiempo en Argentina al igual que en Latinoamérica son prácticamente inexistentes, por lo que este constituye un interesante campo de investigación económica abordado en este estudio. 


\subsection{Las comparaciones de los niveles de MII entre países}

Las investigaciones que, como ésta, obtuvieron estimaciones comparables de los niveles de MII entre países de América Latina son casi inexistentes. Una excepción, probablemente la única, es el estudio desarrollado por Grawe (2004a) que incluye dos países de la región - Ecuador y Perú - además de Nepal, Pakistán, Malasia, Estados Unidos, Canadá, Alemania y Reino Unido. Como el autor implementa el método TwoSample Instrumental Variables (TSIV) en el caso de los cinco países en desarrollo que considera, estas estimaciones aunque comparables entre sí, no lo son con las realizadas para los otros cuatros países desarrollados incluidos. Los resultados obtenidos por Grawe (2004a) sugieren que la persistencia intergeneracional de ingresos es mayor en Ecuador y Perú que en Nepal y Pakistán. Además, mientras en estos dos últimos países, los niveles de MII son mayores en los cuantiles más altos de la distribución, lo contrario se observa en Ecuador y Perú.

Al igual que las investigaciones que analizan la movilidad social intergeneracional en un país en desarrollo, aquellas que realizan comparaciones entre países latinoamericanos consideraron, en su mayoría, como variable de resultado la educación (Dahan y Gaviria, 2001; Andersen, 2001; Gaviria, 2005; Conconi et al., 2008; Ferreira et al., 2013) ${ }^{62}$.

La mayoría de los estudios que estiman y comparan, entre dos o más países, indicadores de movilidad intergeneracional del ingreso lo hicieron para países desarrollados. Entre ellos pueden citarse como antecedentes los de Björklund y Jantti (1997) y Couch y Dunn (1997), mientras el primero compara los niveles de MII entre Suecia y Estados Unidos, el segundo, lo hace entre este último país y Alemania. Los resultados obtenidos por Björklund y Jantti (1997) indican que las EII estimadas para los Estados Unidos son siempre más elevadas que las de Suecia, de manera que éste último país presenta mayores niveles de movilidad intergeneracional y menores niveles de desigualdad. Esto suscita la pregunta respecto de las relaciones entre la igualdad de resultados y la de oportunidades. Por esto, los autores consideran que otras comparaciones internacionales podrían ayudar a explorar esta importante cuestión. En

\footnotetext{
${ }^{62}$ Una revisión reciente de la evidencia empírica sobre movilidad social intergeneracional en América Latina puede encontrarse en Azevedo y Bouillon (2010).
} 
cambio, Couch y Dunn (1997) muestran que los niveles de correlación de ingresos laborales entre padres e hijos observados en Alemania son similares a los estimados para los Estados Unidos.

Entre los estudios relativamente más recientes se encuentran los de Blanden et al. (2005), Brastberg et al. (2007), Vogel (2006), Jäntti et al. (2006), Leigh (2007), Raaum et al. (2007), Lefranc et al. (2010), Ng et al. (2009) y Björklund et al. (2012). Los estudios más amplios, en términos de la cantidad de países comparados, son los de Blanden et al. $(2005)^{63}$, Brastberg et al. (2007) $)^{64}$, Jäntti et al. (2006) ${ }^{65}$ y Raaum et al. $(2007)^{66}$ que incluyen de cuatro a seis países, entre europeos y norteamericanos. En general, los resultados de estas investigaciones sugieren, sorprendentemente para algunos, que la movilidad es menor en los Estados Unidos en comparación con la observada en Reino Unido que, a su vez, es más baja que la estimada en los países nórdicos de Europa como Dinamarca, Finlandia, Noruega y Suecia ${ }^{67}$.

Los restantes estudios mencionados comparan la MII entre dos países, siendo uno de ellos, en la mayoría de los casos, Estados Unidos. Así, Vogel (2006) encuentran que la MII es más limitada en los Estados Unidos que en Alemania. Leigh (2007), por su parte, muestra que Australia presenta más movilidad intergeneracional que Estados Unidos, usando métodos similares de estimación para ambos países. Uno de los pocos estudios que comparan la MII entre un país en desarrollo, Singapur y un país

\footnotetext{
${ }^{63}$ En este estudio se incluyen en el análisis comparativo a Gran Bretaña, Alemania, Estados Unidos, y Canadá.

${ }^{64}$ Brastberg et al. (2007) comparan los patrones de MII en Dinamarca, Finlandia, Noruega, Reino Unido (UK) y Estados Unidos (US). Un aspecto novedoso en este estudio es el análisis y comparación de las no linealidades existentes en la MII. Los resultados obtenidos muestran que los patrones de MII en los países del norte de Europa, a diferencia de los observados en UK y US, son altamente no lineales y convexos, sugiriendo que los hijos que crecen en los hogares más pobres tienen las mismas perspectivas de ingreso adulto que los hijos en los hogares moderamente pobres, pero con un efecto crecientemente positivo del ingreso del padre en los segmentos medios y más altos de la distribución. Este patrón contrasta con la relación casi lineal, entre ingresos de padres e hijos, obtenida en US y UK.

${ }^{65}$ Esta investigación compara los niveles de MII estimados en Dinamarca, Finlandia, Noruega, Suecia, Reino Unido y Estados Unidos.

${ }^{66}$ El estudio de Raaum et al. (2007) presenta evidencia comparable sobre MII para Dinamarca, Finlandia, Noruega, Reino Unido y Estados Unidos. Una contribución destacable de esta investigación es el análisis del efecto del género y el assortative mating en los procesos de transmisión intergeneracional.

${ }^{67}$ Sin embargo, conforme con los resultados obtenidos por Raaum et al. (2007), para las mujeres casadas la MII es aproximadamente uniforme entre los países cuando las estimaciones están basadas en los ingresos laborales de las mujeres y no en sus ingresos familiares. Los autores explican este resultado por la combinación del assortative mating y las respuestas de la oferta laboral femenina en Estados Unidos y el Reino Unido que debilitan la asociación intergeneracional entre el ingreso laboral propio de las mujeres y el de sus padres.
} 
desarrollado, Estados Unidos, es el de Ng et al. (2009). Los resultados muestran que los niveles de movilidad son similares en ambos lo que, según los autores, no es sorprendente pues los dos países tienen similares realidades económicas, sistemas de protección social, regímenes educativos y estructuras laborales. En tanto que, Lefranc et al. (2010) comparando el grado de correlación intergeneracional de ingresos y niveles educativos en Francia y Japón, sostienen que los niveles de MII son mayores en éste último país. Por último, Björklund et al. (2012) exploran algunos mecanismos subyacentes a las diferencias en la transmisión intergeneracional del ingreso entre países, para explicar los mayores niveles de MII observados en Suecia en comparación con Reino Unido. Los autores encuentran que mientras variables como el peso al nacer y la altura en la adolescencia no parecen ser mecanismos importantes, las diferencias entre los países en la performance escolar y el nivel educativo dan cuenta de una parte substancial de las diferencias observadas en la persistencia intergeneracional del ingreso.

A diferencia de los estudios anteriores que se proponen obtener estimaciones comparables, otros realizan comparaciones entre países a partir de un meta-análisis de las estimaciones, principalmente de la EII, disponibles en la literatura empírica como los de Solon (2002), Corak (2006, 2013), Holter (2015) y Blanden (2013). Sin embargo, una clara limitación de este tipo de análisis son las diferencias existentes en las variables de ingreso consideradas, las bases de datos, la selección de la muestra utilizada (en particular, la composición etaria de los pares de padres e hijos considerados), los métodos de estimación, el año en el que se observa el ingreso de padres e hijos (determinados años podrían capturar más el efecto de fluctuaciones en el ciclo económico) que pueden explicar, en parte, la variación en las estimaciones entre países. De allí, la importancia de realizar estudios comparativos que controlen todos estos aspectos, permitiendo asignar una interpretación económica a las diferencias o similitudes encontradas en los niveles de MII entre distintos países.

Un problema que ninguno de los estudios mencionados ha considerado al realizar las comparaciones es la posibilidad de la existencia de una heterogeneidad inobservada producto del momento en el ciclo de vida de los hijos en que se observa el 
ingreso parental. Esta observación realizada recientemente por Muller (2010) ${ }^{68}$ lleva a cuestionar el modelo intergeneracional tradicional de ingreso permanente, dando lugar a un modelo alternativo que será examinado en Chile y Argentina, a partir de un nuevo método de estimación aquí propuesto.

\section{Análisis de los modelos empíricos intergeneracionales del ingreso y sus métodos de estimación}

Como se mencionó en la primera sección, los objetivos de este capítulo son varios. Por un lado, evaluar los métodos disponibles así como el propuesto en esta investigación para estimar la EII, explotando las ventajas de la estructura longitudinal de la EPCASEN. Por otra parte, se busca también comparar los niveles de movilidad intergeneracional del ingreso en Argentina y Chile.

Por esto, a continuación se describen, en primer lugar, los modelos y métodos de estimación propuestos en la literatura para medir la EII, prestando especial atención a los potenciales sesgos que pueden presentar. Para esto se derivan los límites en probabilidad de distintos estimadores utilizados. También, se considera un modelo alternativo recientemente formulado por Muller (2010) que aún no ha sido evaluado empíricamente para otro país. Con este fin, se propone una nueva técnica de estimación de la EII, en el contexto de este modelo, utilizando datos de corte transversal que, en muchos países, como Argentina, son la única fuente de información disponible.

En la comparación de los niveles de MII entre países deben tenerse en cuenta varias cuestiones. En la descripción de cada una de ellas que se realiza a continuación, se resaltan además algunos puntos ignorados o que se consideran tuvieron un tratamiento erróneo en la mayoría de los estudios aplicados.

Por otra parte, resulta importante señalar que, como se estableció en el capítulo anterior, una caracterización más completa de los procesos de MII requiere examinar la distribución del ingreso de los hijos condicional al ingreso de sus padres. Una

\footnotetext{
${ }^{68} \mathrm{Si}$ bien Muller (2008) reconoce que hay varios estudios previos como el de Hertz (2005) que consideran la relevancia de medir el ingreso paterno durante la niñez antes que en cualquier otro período, su discusión está estrictamente desvinculada de la estructura implícita del modelo intergeneracional asumido que sólo permite una relación entre el ingreso permanente de padres e hijos.
} 
herramienta ampliamente utilizada para ello es la matriz de transición intergeneracional. Como ya fue mencionado, otra técnica que puede implementarse para analizar la variación de los niveles de MII a lo largo de la distribución del ingreso es el método QR y también la técnica relativamente novedosa de regresiones por cuantiles no condicionales (UQR). No obstante, las restricciones de los datos con los que se cuenta para analizar la MII, particularmente en Argentina, dificultan realizar este tipo de análisis o uno similar al desarrollado en el capítulo anterior para la MEI. En efecto, la estimación de matrices de transición intergeneracional entre cuantiles de ingresos y de EII a partir de QR o UQR requiere, en principio, contar con información sobre la distribución del ingreso de los padres y de los hijos adultos, en edades centrales del ciclo vital de ambos. Sin embargo, las encuestas disponibles para Argentina no contienen datos del ingreso de los padres o sólo tienen esta información para aquellos hijos que residen con ellos. Si bien, en este caso podría implementarse una estrategia de estimación con variables instrumentales para predecir el ingreso paterno, esta técnica genera un problema de discretización de los datos ${ }^{69}$. Además, el enfoque de matrices de transición del ingreso está basado en una partición arbitraria de la distribución (quintiles o cuartiles) (Bhattacharya y Mazumder, 2011). Asimismo, el análisis formal de los potenciales sesgos presentes en estas estimaciones (probabilidades de transición y EII computadas a partir de QR y UQR) excede los objetivos de este capítulo pero forman parte de una línea de investigación futura. Justamente, uno de los propósitos centrales del capítulo es analizar detenidamente los diferentes métodos propuestos en la literatura para estimar la EII en la media condicional del ingreso de los hijos que es la medida más utilizada para caracterizar los niveles promedio de MII existentes en un país.

\subsection{El modelo intergeneracional de ingreso permanente y el modelo clásico de error de medición}

El modelo estadístico tradicional considerado en la literatura que estima la elasticidad intergeneracional del ingreso (EII) es el siguiente:

\footnotetext{
${ }^{69}$ No obstante, en el contexto de esta investigación se estimaron y analizaron matrices de transición intergeneracionales del ingreso así como EII a partir del método QR y UQR para Argentina. Estos resultados preliminares que, por los problemas metodológicos mencionados no se incluyen aquí, pueden consultarse en Jiménez (2011b, 2012, 2016).
} 


$$
\begin{aligned}
& y_{h i t}=y_{h i}+u_{h i t} \\
& y_{p i s}=y_{p i}+u_{p s} \\
& y_{h i}=\beta y_{p i}+\varepsilon_{h i}
\end{aligned}
$$

donde $y_{\text {hit }}$ es el ingreso corriente del hijo en el año t y $y_{\text {pis }}$ es el ingreso corriente del padre en el año s expresados como una función del ingreso permanente $\left(y_{h i} \mathrm{y} y_{p i}\right)$ que refleja la verdadera capacidad de ingresos a largo plazo y un término de error ( $u_{\text {hit }}=\omega_{\text {hit }}+v_{\text {hit }}$ y $\left.u_{p i s}=\omega_{p i s}+v_{p i s}\right)$ con componentes que capturan shocks transitorios en los ingresos $\left(\omega_{h t} \mathrm{y} \omega_{p s}\right)$ y componentes de ruido blanco $\left(v_{h i t} \mathrm{y} v_{p i s}\right)^{70}$. La ecuación (3) es el modelo intergeneracional de interés que relaciona el ingreso permanente de hijos y padres y $\beta$ es la elasticidad intergeneracional del ingreso (EII), esto es, una medida del grado de persistencia del ingreso permanente de padres e hijos. La EII también puede interpretarse como la fracción de la brecha de ingresos entre padres con distintos niveles de ingresos que, en promedio, persiste a través de las generaciones. En otras palabras, $\beta$ resume en un solo número el grado de inmovilidad generacional del ingreso en una sociedad (Corak, 2004). De esta forma, el nivel de MI o el grado con el cual los ingresos no están asociados entre generaciones es medido a partir de $(1-\beta)$.

Este modelo, definido por las ecuaciones (1)-(2)-(3), asume implícitamente que no hay correlación entre los componentes permanentes y transitorios del ingreso de padres e hijos ${ }^{71}$. De manera que los componentes permanentes capturarán el impacto de factores constantes como la educación, características y atributos genéticos de los padres y cualquier efecto causal del componente permanente del ingreso en sí mismo. Por lo tanto, es importante aclarar, antes de analizar los distintos estimadores propuestos para la EII, que ésta no constituye (ni se espera que sea) una medida del efecto causal del ingreso de los padres en el resultado económico de los hijos. En general, es inevitable que la EII sobrestime cualquier relación causal que exista como resultado de la correlación entre el ingreso de los padres y otras variables explicativas en la ecuación estructural del ingreso (o, en general, del resultado económico) de los hijos. No

\footnotetext{
${ }^{70}$ Se asume para simplificar la notación y sin pérdida de generalidad que los ingresos están escritos como desvíos de las medias (por esto no se incluyen constantes en los modelos).

${ }^{71}$ En el apartado 3.3. se cuestiona este supuesto, considerando un modelo alternativo propuesto por Muller (2010).
} 
obstante, aun así, la EII es una medida relevante de inmovilidad intergeneracional. Pero, debe recordarse que este tipo de modelos deja pendiente la exploración de los determinantes de esa inmovilidad. En términos de la clasificación propuesta por Goldberger (1989), el modelo definido por las ecuaciones (1)-(2)-(3) es del tipo "mecánico" y no "económico" 72 (Muller, 2008).

La estimación por Ordinary Least Squares (OLS) de (3) usando los ingresos corrientes en lugar de los permanentes $\left(y_{h i}\right.$ y $\left.y_{p i}\right)$ produce estimaciones sesgadas de la EII. Formalmente, el límite en probabilidad de la EII estimada por OLS ${ }^{73}$ es:

$$
\operatorname{plim} \hat{\beta}_{O L S}=\beta\left(\frac{\operatorname{var}\left(y_{p}\right)}{\operatorname{var}\left(y_{p}\right)+\operatorname{var}\left(\omega_{p s}\right)+\operatorname{var}\left(v_{p s}\right)}\right)
$$

Para obtener (4), se asume que $\operatorname{Cov}\left(y_{p}, u_{h t}\right)=\operatorname{Cov}\left(y_{h}, u_{p s}\right)=\operatorname{Cov}\left(u_{p s}, u_{h t}\right)=0$. En este caso, la estimación por OLS estará sujeta al tradicional sesgo de atenuación por error de medición en las variables. En particular, teniendo en cuenta que $u_{p i s}=\omega_{p i s}+v_{p i s}$, eso implica asumir que $\omega_{p i s}$ es también un componente de ruido blanco independiente del ingreso permanente. No obstante, como advierten Nybom y Sthuler (2016), el estimador $\hat{\beta}_{O L S}$ estrictamente podría subestimar como sobrestimar la verdadera EII si el término de error incluye un shock transitorio $\left(\omega_{p i s}\right.$ y $\left.\omega_{\text {hit }}\right)$ que podría estar correlacionado con el ingreso permanente.

La mayoría de los primeros estudios empíricos que intentaron estimar la EII, asumieron explícita o implícitamente que $\operatorname{var}\left(\omega_{p s}\right)=\operatorname{var}\left(v_{p s}\right)=0$, es decir, suponían que los estimadores OLS eran consistentes. Algunos, en cambio, reconociendo el problema de error de medición, propusieron como forma de solucionarlo introducir controles por la edad de padres e hijos en la ecuación (3), cuando se utilizaran los ingresos corrientes en lugar de los permanentes. Otros adoptaron la estrategia de estimar, primero, una regresión de los ingresos corrientes de padres e hijos en un

\footnotetext{
${ }^{72}$ Modelos intergeneracionales del tipo "económico", es decir, que analicen los factores subyacentes son, por ejemplo, los desarrollados por Becker y Tomes (1979) o Solon (2004. El problema de considerar modelos mecánicos, como advierte Muller (2008), surge cuando se da una atención insignificante a los posibles canales causales porque esto podría llevar a que el modelo "mecánico" sobre el cual se basan las estimaciones de la EII resulte defectuoso o incorrecto. Más adelante, se retoma este punto al discutir un modelo intergeneracional alternativo.

${ }^{73}$ En el Anexo B se puede consultar la derivación de la ecuación (4).
} 
polinomio cuadrático o cuártico de sus respectivas edades y usar los residuos de estas regresiones para calcular la EII.

Sin embargo, una segunda generación de investigaciones demostró que ninguna de esas soluciones eliminaba el problema de error de medición en el ingreso permanente del padre. Entonces, las EII así estimadas aún presentaban un sesgo de atenuación ${ }^{74}$. Las dos soluciones propuestas y más utilizadas en la literatura para este problema clásico de error en las variables fueron la técnica de promediar los ingresos del padre a lo largo de un período T determinado (PT) y el método Instrumental Variable (IV).

Usando un promedio a lo largo de $\mathrm{T}$ años del ingreso del padre como variable independiente en (3), el límite en probabilidad de la EII estimada es ${ }^{75}$ :

$$
\operatorname{plim} \hat{\beta}_{P T}=\beta\left(\frac{\operatorname{var}\left(y_{p}\right)}{\operatorname{var}\left(y_{p}\right)+\left(\frac{1}{T}\right) \operatorname{var}\left(\omega_{p}\right)+\left(\frac{1}{T}\right) \operatorname{var}\left(v_{p}\right)}\right)
$$

Es decir, este procedimiento reduce el sesgo en la estimación de $\beta$ pues el promedio elimina el componente transitorio de los ingresos del padre. Cuanto mayor sea el número de períodos, T, a lo largo de los cuales se tome el promedio, mayor es la reducción en el sesgo (Zimmerman, 1992). Pero, si, como advierte Solon (1992) y Zimmerman (1992), el término que captura el efecto de shock transitorio en el ingreso sigue un proceso $\mathrm{AR}(1)$, de forma tal que

$$
\omega_{p i s}=\delta \omega_{p i s-1}+\xi_{p i s}
$$

Entonces, el límite en probabilidad de la EII estimada será:

$$
\operatorname{plim} \hat{\beta}_{P T}=\beta\left(\frac{\operatorname{var}\left(y_{p}\right)}{\operatorname{var}\left(y_{p}\right)+\left(\frac{1}{T}\right) \alpha \cdot \operatorname{var}\left(\omega_{p}\right)+\left(\frac{1}{T}\right) \operatorname{var}\left(v_{p}\right)}\right)
$$

\footnotetext{
${ }^{74}$ Jenkins (1987) es uno de los primeros en dudar sobre los méritos de esas estrategias de solución. Luego, Haider y Solon (2006) mostraron que la práctica común de controlar por la tendencia central de crecimiento en los ingresos en la población no es suficiente porque la variación heterogénea alrededor de la tasa de crecimiento promedio de los ingresos sesgaría las estimaciones de la EII. Específicamente, la inclusión de controles por edad y experiencia en una regresión media como (3) controlará solamente por los efectos promedios de la edad o la experiencia y no capturará la variación heterogénea del crecimiento en los ingresos alrededor de la tasa de crecimiento promedio (Stuhler, 2010).

${ }^{75}$ En el Anexo B se encuentra la derivación de la ecuación (5) y la ecuación (7).
} 
donde $\alpha=1+2 \delta\left(\frac{T-\delta T-1+\delta^{T}}{T(1-\delta)^{2}}\right)$

Por lo tanto, el efecto de reducción del sesgo de atenuación como resultado de promediar el ingreso a lo largo de un período T es mitigado si existe una correlación entre los componentes transitorios. En definitiva, si el modelo (1) a (3) es el correcto, la estimación por OLS ofrecerá, muy probablemente, un estimador que subestima la verdadera EII.

En cambio, conforme con los supuestos asumidos por Solon (1992), el método IV produciría un estimador inconsistente que sobrestima $\beta$. De manera que el estimador OLS y IV de la EII en (3), ofrecerían un límite inferior y superior, respectivamente, para $\beta$. Sin embargo, el argumento esgrimido por Solon $(1989,1992)$ y retomado por Zimmerman $(1992)^{76}$ para justificar que el estimador IV sobrestimaría la verdadera EII podría no ser totalmente adecuado. Para ver esto formalmente, siguiendo a Stuhler (2010) y Nybom y Sthuler (2016), dado el modelo (1) a (3), supongamos que el ingreso permanente de padres es una función de un vector de características o variables explicativas $\left(Z_{p i}\right)$ de forma tal que:

$$
y_{p i}=\gamma Z_{p i}+\varepsilon_{p i}
$$

Si el vector $Z_{p i}$ incluye variables, como la educación de los padres $E_{p i}$, que pueden esperarse que tengan un efecto directo sobre el ingreso de los hijos, además del efecto indirecto que puedan tener a través de su correlación con el ingreso del padre, entonces el ingreso permanente de los hijos puede ser escrito como:

$$
y_{h i}=\delta_{1} y_{p i}+\delta_{2} E_{p i}+\varepsilon_{h i}
$$

Si se estima la ecuación (3) usando el ingreso corriente observado en t como proxy del ingreso de largo plazo para hijos $\left(y_{h i t}=y_{h i}+u_{h i t}\right)$ y la predicción del ingreso

\footnotetext{
76 Estos son los dos estudios seminales de una segunda generación de estudios empíricos que implementaron las estrategias de estimación allí propuestas (los métodos PT y IV) para corregir el problema de error de medición del ingreso permanente del padre.
} 
permanente de los padres $\left(y_{p i s}=y_{p i}+u_{p i s}\right)$ por el método IV con la educación como instrumento, el límite en probabilidad del estimador IV de la $\mathrm{EII}^{77}$ es:

$$
p \lim \hat{\beta}_{I V}=\beta+\delta_{2} \sigma_{E_{p}}\left[\frac{1-\operatorname{Corr}\left(E_{p}, y_{p}\right)^{2}}{\operatorname{Corr}\left(E_{p}, y_{p}\right) \sigma_{y_{p}}}\right]+\frac{\operatorname{Cov}\left(E_{p}, u_{h t}\right)}{\operatorname{Cov}\left(E_{p}, y_{p s}\right)}
$$

donde $\sigma_{E_{p}}=\sqrt{\operatorname{Var}\left(E_{p}\right)}$ y $\sigma_{y_{p}}=\sqrt{\operatorname{Var}\left(y_{p}\right)}$. A partir de (10) se puede observar que el estimador IV de $\beta$ sobrestimará la verdadera EII, como afirman Solon (1992) y Zimmerman (1992) siempre que se cumplan las siguientes condiciones:

(i) $\operatorname{Cov}\left(E_{p}, u_{h t}\right) \geq 0 \mathrm{y} \operatorname{Cov}\left(E_{p}, u_{p s}\right) \geq 0$, es decir, la educación de los padres no esté correlacionada (o tenga una correlación positiva) con el error de medición del ingreso permanente conformado por un componente que captura los shocks transitorios y un ruido blanco conforme con (1) y (2);

(ii) $\delta_{2} \geq 0$, es decir, que la educación del padre tenga un efecto no-negativo en el ingreso de los hijos;

(iii) $0<\operatorname{Corr}\left(E_{p}, y_{p}\right)<1$, esto es, que la correlación entre el ingreso y la educación de los padres esté acotada entre cero y uno.

Si bien, los supuestos (ii) y (iii) son razonables, el primero (i), podría no cumplirse. Esta cuestión está asociada con el denominado life-cycle bias que se examinará en el siguiente apartado. Sin embargo, si el primer supuesto se cumple y, además, en línea con los hallazgos de Sewell y Hauser (1975), Corcoran et al. (1992), Mazumder (2005), el efecto directo de la educación del padre sobre el ingreso del hijo no es estadísticamente distinto de cero, una vez que se controla por el ingreso del padre promediado durante varios años, la estimación IV de la EII sería consistente. En definitiva, si el modelo definido en (1), (2) y (3) es el correcto, la estimación de las EII, con fines comparativos entre dos o más países, debería realizarse a partir de la técnica $\mathrm{PT}$, si se dispone de información del ingreso del padre para el mismo período $\mathrm{T}$ o mediante el método IV, utilizando las mismas variables instrumentales, lo que permitiría disponer de una mejor medida del ingreso permanente y mejores

\footnotetext{
${ }^{77}$ En el Anexo B puede consultarse la derivación de la ecuación (10).
} 
estimaciones de EII, mayores que las que se obtendría estimando (3) simplemente por OLS.

\subsection{El método de estimación con variables instrumentales utilizando dos muestras}

Un problema adicional que se presenta para estimar (3), en el caso de varios países, como Argentina, es la falta de información sobre el ingreso de los padres para todos los individuos incluidos en la base de datos disponibles. Una solución para esto consiste implementar una estrategia de estimación alternativa que constituye una extensión del método IV tradicional en la que los momentos muestrales necesarios para estimar el coeficiente de interés $(\beta)$ son tomados de dos muestras diferentes. Se utiliza, entonces, una muestra principal en la que se observa el ingreso de los hijos y ciertas características de los padres (generalmente, su educación u ocupación) y una muestra secundaria o auxiliar con información del ingreso y las mismas características para un conjunto de individuos $^{78}$. Así, es posible predecir el ingreso de los padres a partir de los parámetros estimados a partir de la muestra secundaria y de las características de los padres observadas o reportadas por los hijos en la muestra principal. Esos ingresos predichos son usados en la segunda etapa del procedimiento como regresores del modelo del ingreso de los hijos que se estima con la muestra principal.

Este método denominado Two Sample Instrumental Variable (TSIV) o TwoSample Two-Stage Least Squares (TS2SLS), fue propuesto por primera vez por Klevmarken (1982) y aplicado originalmente por Angrist y Krueger (1992) así como por Arellano y Meghir (1992) ${ }^{79}$. Aunque este estimador puede ser considerado como una clase especial dentro de los estimadores IV. También puede pensarse en esta técnica

\footnotetext{
${ }^{78}$ En general, esta segunda muestra corresponde a un momento en el pasado en el que los padres eran más jóvenes. Esto permite obtener una predicción del ingreso de los padres en edades centrales y reducir, así, el denominado lyfe-cycle bias que se describe en la próxima sección.

79 Arrellano y Meghir (1992) analizan las propiedades asintóticas del estimador TSIV. En tanto que, Inoue y Solon (2008) hacen lo propio con el estimador TS2SLS. El método TS2SLS es similar al 2SLS excepto porque las estimaciones de la primera etapa son tomadas de una muestra diferente que las de la segunda etapa. Dos propiedades interesantes de este último estimador son que el estimador TS2SLS puede tener menos sesgo que el convencional 2SLS conforme con Angrist y Pishchke (2009) y es más eficiente que el TSIV.
} 
como un procedimiento de imputación cold-deck (Nicoletti y Ermisch, 2008) o como parte del enfoque para generar regresores inobservado de Murphy y Topel (1985) (Jerrim et al., 2014).

Según Björklund y Jäntti (1997), el estimador TSIV es equivalente al estimador IV tradicional si: (i) las dos muestras son de la misma población, es decir, si los momentos muestrales tienen los mismos valores límites y (ii) las características de los padres reportadas por los hijos no incluyen errores de medición.

Siguiendo a Jerrim et al. (2014), supongamos que se dispone de una muestra auxiliar secundaria $\mathrm{J}$ que cumple las siguientes condiciones: (i) contiene una medida del ingreso permanente de $\operatorname{los}$ entrevistados ${ }^{80}$, (ii) es obtenida de la misma población que la muestra principal y (iii) contiene las mismas variables instrumentales $\mathrm{Z}$ que la muestra principal.

Entonces usando esta muestra secundaria $\mathrm{J}$ es posible estimar el siguiente modelo de regresión por OLS:

$$
y_{j}=\gamma Z_{j}+v_{j}
$$

Usando los coeficientes estimados es posible predecir el ingreso permanente del padre en la muestra principal I:

$$
\hat{y}_{p i}=\hat{\gamma} Z_{p i}
$$

De esta forma, la ecuación (3) ahora puede ser estimada a partir de:

$$
y_{h i}=\beta \hat{y}_{p i}+\varepsilon_{h i}
$$

Como ya fue señalado, las dos variables instrumentales más comúnmente utilizadas son la educación y la ocupación. Sin embargo, ambas variables probablemente sean endógenas, es decir, influyan en el ingreso de los hijos. En este caso, el ingreso de los hijos estará dado por:

$$
y_{h i}=\lambda_{1} y_{p i}+\lambda_{2} \hat{y}_{p i}+u_{h i}(14)
$$

\footnotetext{
${ }^{80}$ En la práctica suele utilizarse como medida del ingreso permanente el ingreso promedio a lo largo de varios años. En la próxima sección se analiza el límite en probabilidad del estimador TS2SLS para el caso en que no se cumple esta condición, es decir, cuando no se observa el ingreso permanente sino el ingreso corriente de padres e hijos.
} 
donde $\lambda_{1}$ es el impacto directo del ingreso permanente del padre sobre el ingreso del hijo y $\lambda_{2}$ es el efecto del ingreso predicho del padre sobre el ingreso de los hijos. Entonces, realizando una derivación similar a la desarrollada para el estimador IV y suponiendo que $\operatorname{Cov}\left(\hat{y}_{p}, u_{h}\right)=0$, el límite en probabilidad del estimador TS2SLS de la $\mathrm{EII}^{81}$ es:

$$
\operatorname{plim} \beta_{T S 2 S L S}=\beta+\lambda_{2} \sigma_{y_{p}}\left[\frac{1-\operatorname{Corr}\left(\hat{y}_{p}, y_{p}\right)^{2}}{\operatorname{Corr}\left(\hat{y}_{p}, y_{p}\right) \sigma_{y_{p}}}\right]
$$

A partir de la ecuación (15) y de la (10) se aprecia la similitud entre el límite en probabilidad de los estimadores IV y TSIV (o 2SLS y TS2SLS), en particular cuando el instrumento utilizado para predecir el ingreso paterno es su educación. Asimismo, se deduce que $\hat{\beta}_{\text {TSTSLS }}$ sobrestimará $\beta$ siempre que:

(i) $\lambda_{2}>0$, es decir, que el efecto directo de las características paternas utilizadas como instrumento para predecir su ingreso sobre el ingreso del hijo sea positivo. Cuando $Z_{p i}=E_{p i}$, esto implica, asumir que el efecto de la educación del padre (ponderada por su retorno estimado $\gamma$ ) sobre el ingreso del hijos es positivo $\mathrm{y}$

(ii) $0 \leq \operatorname{Corr}\left(\hat{y}_{p}, y_{p}\right)<1$, esto es, que la correlación entre la educación del padre (ponderada por su retorno) y el ingreso paterno sea positiva pero no igual a 1.

Siguiendo a Jerrim et al. (2014), es posible obtener una expresión más simple para el límite en probabilidad de $\hat{\beta}_{\text {TSTSLS }}$, bajo el supuesto de que la covarianza entre el ingreso predicho del padre y el real es igual a la covarianza entre el ingreso predicho del padre y él mismo. En este caso, el límite en probabilidad del estimador sería:

$$
\operatorname{plim} \hat{\beta}_{T S T S L S}=\beta+\lambda_{2}\left(1-R^{2}\right)(16)
$$

\footnotetext{
${ }^{81}$ En el Anexo B puede consultarse la derivación de las ecuaciones (15) y (16).
} 
donde $\mathrm{R}^{2}$ es la varianza explicada en el modelo de la primera etapa (11). Por lo tanto, la inconsistencia de $\hat{\beta}_{\text {TSTSLS }}$ es igual a $\lambda_{2}\left(1-R^{2}\right)$. A partir de la ecuación (16) pueden derivarse varias conclusiones:

(i) En la medida en que $0<R^{2} \leq 1$, la varianza del ingreso predicho del padre debe ser menor o igual a la varianza del ingreso real del padre: $0<\operatorname{Var}\left(\hat{y}_{p}\right) \leq \operatorname{Var}\left(y_{p}\right)$.

(ii) Si la varianza del ingreso predicho del padre fuera igual a la varianza del ingreso real del padre entonces $R^{2}=1$ y la inconsistencia de $\hat{\beta}_{\text {TSTSLS }}$ se reduce a cero. Entonces, la inconsistencia de $\hat{\beta}_{\text {TSTSLS }}$ depende, en parte, de la estimación incorrecta de la variabilidad en el ingreso predicho del padre.

(iii) Si las variables $\mathrm{Z}$ son exógenas con respecto al ingreso del hijo, entonces $\lambda_{2}=0$ y $\hat{\beta}_{T S T S L S}$ es consistente. Pero, como se mencionó previamente, cuando se utiliza como variable instrumental $(Z)$ para predecir el ingreso del padre su educación, es probable que $\lambda_{2}>0$, es decir, que el efecto directo de la educación del padre en el ingreso del hijo sea positivo. Entonces, bajo los supuestos de que $\lambda_{2}>0$ y $R^{2}<1, \hat{\beta}_{\text {TSTSLS }}$ sobrestimará la verdadera EII.

(iv) La magnitud del sesgo positivo disminuye a media que la varianza del ingreso predicho del padre se acerca a la varianza del ingreso real del padre, $\operatorname{Var}\left(\hat{y}_{p}\right) \rightarrow \operatorname{Var}\left(y_{p}\right)$.

(v) La inclusión de variables adicionales para incrementar el $R^{2}$ de la ecuación de la primera etapa puede afectar simultáneamente $\lambda_{2}$. Entonces, incluir variables endógenas en $\mathrm{Z}$ podría incrementar $\lambda_{2}$ de tal forma que compense cualquier cambio del $R^{2}$ en la primera etapa.

Con el objetivo de obtener estimaciones comparables y dadas las limitaciones informativas existentes para Argentina, se implementa esta estrategia de estimación con variables instrumentales en dos muestras, utilizando como principal instrumento la educación de los padres. 


\subsection{El efecto del life-cycle bias}

Otro punto a tener en cuenta cuando se comparan EII es que los datos en diferentes países incluyen muestras de pares e hijos de distintas edades. Varios estudios (Mazumder, 2005; Grawe, 2006; Heider y Solon, 2006; Dunn, 2007; Núñez y Miranda, 2011, entre otros) han investigado los efectos de cambiar las edades en las cuales los ingresos de los hijos y/o de los padres son observados. Los resultados obtenidos en estas investigaciones indican que las estimaciones de la persistencia intergeneracional medida con la EII varían con la edad en la cual el ingreso de los padres e hijos son medidos. Este problema es denominado en la literatura como "life-cycle bias".

Formalmente, siguiendo a Haider y Solon (2006), las ecuaciones (1) y (2) deberían adoptar una forma más general para incorporar el hallazgo empírico documentado por las investigaciones previamente mencionadas. Suponiendo que los ingresos de padres e hijos son medidos en una edad particular, $a$ que no necesariamente es la misma para ambos, se tiene que:

$$
\begin{aligned}
& y_{\text {hia }}=\lambda_{a} y_{h i}+\omega_{\text {hia }}+v_{\text {hia }} \\
& y_{\text {pia }}=\mu_{a} y_{p i}+\omega_{\text {pia }}+v_{\text {pia }}
\end{aligned}
$$

Comparando (17) y (18) con (1) y (2) es claro que la principal diferencia radica en el coeficiente de pendiente correspondiente a la regresión del ingreso corriente en el ingreso permanente que no se asume igual a uno, como en el modelo clásico de errores en las variables, originado en el planteo de Friedman (1957). Esto es así, porque, como advierten Haider y Solon (2006), existen razones para sospechar que ese coeficiente de pendiente en estas regresiones varía sistemáticamente a lo largo del ciclo de vida y no es, en general, igual a uno como resultado del cambio en la varianza de los ingresos.

Suponiendo que los términos de error no están correlacionados entre sí ni con el ingreso permanente ${ }^{82}$, el límite en probabilidad del estimador OLS de la EII ${ }^{83}$ en el modelo (17)-(18)-(3) es el siguiente:

\footnotetext{
${ }^{82}$ Este puede ser un supuesto fuerte considerando que padres e hijos pueden tener similares trayectorias laborales (Black y Devearux, 2011).

${ }^{83}$ En el Anexo B puede consultarse la derivación de la ecuación (19).
} 


$$
p \lim \beta_{\text {OLS }}=\beta\left(\frac{\lambda_{a} \mu_{a} \operatorname{var}\left(y_{p}\right)}{\mu_{a}^{2} \operatorname{var}\left(y_{p}\right)+\operatorname{var}\left(\omega_{p}\right)+\operatorname{var}\left(v_{p}\right)}\right)=\beta \lambda_{a} \theta_{a}
$$

donde $\theta_{a}=\left(\frac{\mu_{a} \operatorname{var}\left(y_{p}\right)}{\mu_{a}^{2} \operatorname{var}\left(y_{p}\right)+\operatorname{var}\left(\omega_{p}\right)+\operatorname{var}\left(v_{p}\right)}\right)$

Como señalan Black y Deveraux (2011) este simple modelo tiene diversas implicancias:

(i) Si $\lambda_{a}=\mu_{a}=1$, el modelo coincide con el modelo clásico de error en las variables y, conforme se mostró previamente, el estimador OLS de la EII sufriría de sesgo de atenuación.

(ii) Aun cuando $\lambda_{a}=\mu_{a}=1$, la magnitud del sesgo de atenuación puede depender de la edad del padre, en la medida en que $\operatorname{var}\left(\omega_{p}\right)$ pueda en sí misma depender de la edad. Baker y Solon (2003) y Mazumder (2005) ofrecen evidencia de que $\operatorname{var}\left(\omega_{p}\right)$ varía a lo largo del ciclo de vida y alcanza su mínimo alrededor de los 40 años.

(iii) Si $\theta_{a}>1$, lo que es más probable a medida que $\mu_{a}$ es menor, el sesgo de $\beta_{\text {OLS }}$ no necesariamente es un sesgo de atenuación.

(iv) A diferencia de lo que se asume en el modelo clásico de error en las variables, en este caso el error de medición en la variable dependiente $y_{s}$ produce un sesgo en la medida en que $\lambda_{a} \neq 1$ y el tamaño del sesgo depende potencialmente de la edad en la que es medido el ingreso del hijo.

Este es un punto importante a tener en cuenta en las comparaciones entre países porque en algunos las limitaciones de los datos disponibles hacen que el ingreso de los padres sea medido relativamente tarde en su ciclo de vida, mientras que el ingreso de los hijos es medido a edades bastante más jóvenes. Haider y Solon (2006) así como Böhlmark y Lindquist (2006) estimaron $\lambda_{a}$ y $\theta_{a}$ llegando a conclusiones similares. Las estimaciones de $\lambda_{a}$ se incrementan con la edad y son cercanas a 1 cuando los individuos alcanzan los 30 años, permanecen en aproximadamente en este valor hasta pasados sus 40 años, pero disminuyen hacia fines de la década de los 50 años, alcanzando valores 
aproximadamente cercanos a 0.6. Por su parte, las estimaciones de $\theta_{a}$ (también denominada reliability ratio o signal-to-noise ratio) indican que este coeficiente toma valores cercanos a 0.20 a $\operatorname{los} 20$ años y se incrementa a $0.6-0.7$ a los 30 años. Permanece en este valor hasta pasados los 40 años y disminuye lentamente hasta $0.4-0.5$ hacia fines de la década de los 50 años.

En definitiva, estos resultados sugieren que tanto el ingreso de padres como de hijos deberían medirse óptimamente en las edades centrales de su ciclo de vida. Sin embargo, incluso así el factor de atenuación asociado con el error de medición en el ingreso de los padres $\theta_{a}$ alcanzaría el valor de 0.7 , por tanto, aún existiría un sesgo de atenuación en el estimador OLS de la EII.

Por otra parte, si el modelo correcto es el dado por las ecuaciones (3), (8), (9), (17) y (18), asumiendo que $\operatorname{Cov}\left(E_{p}, u_{p a}\right)=0$, el límite en probabilidad del estimador IV de la EII $^{84}$ sería:

$$
p \lim \beta_{I V}=\frac{\lambda_{a}}{\mu_{a}} \beta+\lambda_{a} \delta_{2} \sigma_{E_{p}}\left[\frac{1-\frac{1}{\mu_{a}} \operatorname{Corr}\left(E_{p}, y_{p a}\right)^{2}}{\operatorname{Corr}\left(E_{p}, y_{p a}\right) \sigma_{y_{p}}}\right]+\frac{\operatorname{Cov}\left(E_{p}, u_{h a}\right)}{\operatorname{Cov}\left(E_{p}, y_{p a}\right)}
$$

Cuando en el modelo a estimar está conformado por las ecuaciones (3), (8), (9), (17) y (18), con una derivación similar a la del estimador IV y asumiendo que $\operatorname{Cov}\left(\hat{y}_{p}, \varepsilon_{h}\right)=0$ y $\operatorname{Cov}\left(\hat{y}_{p}, u_{p a}\right)=0$, el límite en probabilidad del estimador TSTSLS sería:

$$
p \lim \beta_{T S 2 S L S}=\frac{\lambda_{a}}{\mu_{a}} \beta+\lambda_{a} \lambda_{2} \sigma_{\hat{y}_{p}}\left[\frac{1-\frac{1}{\mu_{a}} \operatorname{Corr}\left(\hat{y}_{p}, y_{p a}\right)^{2}}{\operatorname{Corr}\left(\hat{y}_{p}, y_{p a}\right) \sigma_{y_{p}}}\right]+\frac{\operatorname{Cov}\left(\hat{y}_{p}, u_{h a}\right)}{\operatorname{Cov}\left(\hat{y}_{p}, y_{p a}\right)}
$$

A partir de las ecuaciones (20) y (21) se deduce que tanto el estimador IV como el estimador TS2SLS, en este caso, pueden sobrestimar como subestimar la verdadera EII. Sin embargo, según los resultados obtenidos por Haider y Solon (2006) así como

\footnotetext{
${ }^{84}$ En el Anexo B puede consultarse la derivación de las ecuaciones (20) y (21). Allí se muestran también los límites en probabilidad del estimador IV y del estimador TSIV cuando no se cumplen los supuestos mencionados en el texto.
} 
Böhlmark y Lindquist (2006), si el ingreso corriente de hijos y padres es observado en sus edades centrales, esto es, cuando tienen de 30 a 45 años aproximadamente, $\lambda_{a}$ y $\mu_{a}$ toman valores muy cercanos a uno, entonces el life-cylcle bias desaparece o disminuye. A partir de (20) se deduce que, en este caso, el estimador IV sobrestimaría la EII siempre que: (i) $\delta_{2}>0$, (ii) $0<\operatorname{Corr}\left(E_{p}, y_{p a}\right)<1$ y (iii) $\operatorname{Cov}\left(E_{p}, u_{h a}\right)=\operatorname{Cov}\left(E_{p}, y_{p a}\right)=0$. De forma similar, a partir de la ecuación (21) se deduce que el estimador TS2SLS sobrestimaría la EII siempre que: (i) $\lambda_{2}>0$, teniendo en cuenta la ecuación (13), $\hat{y}_{p i}=\hat{\gamma} Z_{p i}$ y si $Z_{p i}=E_{p}$ esto implica asumir que el efecto directo de la educación del padre en el ingreso del hijo sea positivo, (ii) $0<\operatorname{Corr}\left(\hat{y}_{p}, y_{p a}\right)<1$ y (iii) $\operatorname{Cov}\left(\hat{y}_{p}, y_{p a}\right) \geq 0 \mathrm{y} \operatorname{Cov}\left(\hat{y}_{p}, u_{h a}\right) \geq 0$

Para el cumplimiento de este último supuesto también es relevante que el ingreso de hijos y padres sea medido en sus edades centrales. En efecto, como advierte Sthuler (2010), el hecho que los individuos altamente educados tiendan a tener un mayor crecimiento en los ingresos a lo largo del ciclo de vida complica la interpretación del estimador IV. Si se emplean datos que sólo contienen información del ingreso corriente de los hijos en su juventud y el de los padres en edades más avanzada, el uso de este ingreso corriente como proxy del ingreso permanente o de largo plazo, subestimará el ingreso permanente de los hijos altamente educados y sobrestimará el de los padres, incluso aunque se controle por la tendencia central del crecimiento de los ingresos en la población. Si el logro educativo está correlacionado entre las generaciones se tendrá que $E\left(u_{p} \mid E_{p}=\right.$ alta $)>0$ y $E\left(u_{h} \mid E_{p}=\right.$ alta $)<0$. A partir de esto, con el correspondiente argumento para los individuos menos educados, se seguiría que $\operatorname{Cov}\left(E_{p}, u_{p}\right)>0 \mathrm{y}$ $\operatorname{Cov}\left(E_{p}, u_{h}\right)<0$. En este caso, aun cuando $\lambda_{a}=\mu_{\alpha}=1$, los últimos dos términos en (21) - así como en (20) - pueden ser negativos y el estimador IV no necesariamente será un límite superior del verdadero parámetro $\beta$. 


\subsection{Un modelo intergeneracional alternativo y un nuevo método de estimación}

El modelo intergeneracional tradicionalmente considerado en la literatura sobre MII, así como sus generalizaciones antes descriptas, está basado en el concepto de ingreso permanente y en el supuesto de que esta es la única variable relevante cuando se evalúa la persistencia de ingresos entre generaciones. Esto excluye implícitamente otra clase de modelos estructurales como aquellos que incorporen la posibilidad de que el ingreso de los padres en los primeros años de la niñez tenga una contribución diferencial a la EII o que el efecto de las restricciones crediticias de los padres sobre los ingresos posteriores de los hijos sea diferente en determinadas edades de los hijos. Si estos supuestos son correctos, las estimaciones de la EII podrían variar debido a heterogeneidad inobservada previamente no tomada en consideración. Para contemplar esta posibilidad, Muller (2010) propuso la siguiente modificación de la regresión intergeneracional estándar (3) que permite que el ingreso permanente de los hijos sea una función de las desviaciones del ingreso permanente de los padres $\left(u_{p i q}\right)$ :

$$
y_{h i}=\beta y_{p i}+\sum_{q} \beta_{q} u_{p i q}+\varepsilon_{h i}
$$

donde $u_{p i q}=y_{p i q}-\beta y_{p i}=\omega_{p i a}+v_{p i a} \mathrm{y} \mathrm{q} \in \mathrm{Q}, \mathrm{s} \in \mathrm{S}, \mathrm{Q} \subset \mathrm{S}$.

Los años que conforman la niñez y en el que es medido el ingreso de los padres son indexados por q y s respectivamente. El coeficiente $\beta$ puede variar de forma tal que el ingreso transitorio puede tener diferentes impactos dependiendo de la etapa de la niñez en que ocurra.

Como el ingreso permanente es el mismo en cada año, la EII en este modelo está ahora definida como la suma del efecto del ingreso permanente $(\beta)$ y un promedio de $\operatorname{los} \beta_{q}$ :

$$
B^{*}=\frac{\beta T+\sum_{q} \beta_{q}}{T}=\beta+\frac{\sum_{q} \beta_{q}}{T}
$$

Cuando $\beta_{q}=0, \forall \mathrm{q}$, el modelo se reduce al modelo tradicional definido en (1), (2) y (3). Si el modelo alternativo es el correcto, un punto importante en su estimación es considerar explícitamente si el ingreso de los padres es observado durante la niñez (y 


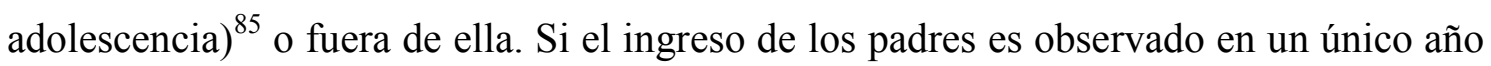
y dentro del período de la niñez (y adolescencia) del hijo, el límite en probabilidad del estimador OLS está dado por $^{86}$ :

$$
p \lim B_{O L S}^{*}=\left(\frac{\beta \operatorname{var}\left(y_{p}\right)+\operatorname{var}\left(\omega_{p}\right) \sum_{q} \beta_{q} \delta^{|q-k|}+\beta_{k} \operatorname{var}\left(v_{p}\right)}{\operatorname{var}\left(y_{p}\right)+\operatorname{var}\left(\omega_{p}\right)+\operatorname{var}\left(v_{p}\right)}\right)
$$

En cambio, si el ingreso de los padres es observado en un único año (k) pero fuera de la niñez (y adolescencia) del hijo, se tiene:

$$
p \lim \hat{B}_{O L S}^{*}=\left(\frac{\beta \operatorname{var}\left(y_{p}\right)+\operatorname{var}\left(\omega_{p}\right) \sum_{q} \beta_{q} \delta^{|q-k|}}{\operatorname{var}\left(y_{p}\right)+\operatorname{var}\left(\omega_{p}\right)+\operatorname{var}\left(v_{p}\right)}\right)
$$

El segundo término en el numerador de (20) y (21) representa la suma de los coeficientes sobre el ingreso transitorio para cada año de la niñez, ponderados por $\delta^{|q-k|}$ que es una función de la correlación $(\delta)$ en el componente transitorio del ingreso del padre $\left(\omega_{p}\right)$ y de la proximidad entre ese año y el año en el que se observa el ingreso paterno $|q-k|$. Si no existiera correlación serial en el ingreso transitorio, $\delta=0$ y el ingreso es observado en un año fuera de la niñez y adolescencia del hijo, el plim en (21) sería igual al definido en (4), de manera que no se capturaría nada del impacto del ingreso transitorio. En cambio, si el ingreso es observado en un año perteneciente a la niñez y adolescencia, el impacto del componente de ruido blanco del ingreso paterno ( $v_{p}$ ) para el año particular usado es parte del coeficiente calculado. Además, en (20), como k está más cercano a q, el segundo término será mayor que el término equivalente en (21). Por lo tanto, las estimaciones de la EII basadas en ingresos observados dentro de la niñez y adolescencia de los hijos deberían ser mayores que las que usan ingresos fuera de ese período. Entonces, la recomendación para el análisis empírico en el que se usa procedimientos de estimación basados en el ingresos de los padres observados en un único año es usar los ingresos percibidos durante la niñez y adolescencia de los hijos y si esto no es factible, al menos tratar de medir los ingresos en un año que sea lo más

\footnotetext{
${ }^{85}$ Muller (2010) supone que la niñez (y adolescencia) se extiende desde que el niño está en el seno materno hasta que alcanza 20 años de edad. En realidad, esta es una cuestión que, en este contexto, debe resolverse empíricamente.

${ }^{86}$ Los detalles de la derivación de los plim del estimador OLS de la EII bajo este modelo alternativo pueden consultarse en Muller (2008).
} 
cercano posible a ese período. Además, como el objetivo es también comparar las estimaciones entre países, Chile y Argentina, en este caso, el ingreso del padre en ambos países debería ser medido en el mismo período de la niñez o adolescencia del hijo.

Según Muller (2008, 2010), los métodos de estimación disponibles en la literatura no permiten estimar correctamente (23). Sin embargo, bajo determinados supuestos, es posible derivar un límite inferior y superior que contenga la verdadera EII (B*). En este caso, las derivaciones ofrecidas por Muller (2008) sugieren que la utilización de un promedio del ingreso corriente de los padres en múltiples años de la niñez o adolescencia de los hijos podría sobrestimar el verdadero coeficiente, ofreciendo un límite superior para la EII verdadera. Formalmente, el límite en probabilidad de la EII estimada considerando como variable explicativa el promedio del ingreso de los padres durante todos los años relevantes de la niñez o adolescencia de los hijos (T), denotada por $\hat{B}_{P T}^{*}$ es:

$$
p \lim \hat{B}_{P T}^{*}=\frac{\beta \operatorname{var}\left(y_{p}\right)+\left(\frac{1}{T}\right) \operatorname{var}\left(\omega_{p}\right) \sum_{q} \beta_{q} \Lambda_{q}+\left(\frac{1}{T}\right) \sum_{q} \beta_{q} \operatorname{var}\left(v_{p}\right)}{\operatorname{var}\left(y_{p}\right)^{2}+\left(\frac{1}{T}\right) a \operatorname{var}\left(\omega_{p}\right)^{2}+\left(\frac{1}{T}\right) \operatorname{var}\left(v_{p}\right)^{2}}
$$

donde $\Lambda_{q}=\left(\frac{1+\delta-\delta^{|q-r|+1}-\delta^{|q-m|+1}}{1-\delta}\right)$ y $\alpha=1+2 \delta\left(\frac{T-\delta T-1+\delta^{T}}{T(1-\delta)^{2}}\right)$

Siendo $\boldsymbol{r}$ y $\boldsymbol{m}$ el primer y último año usado en el promedio, que se supone, coinciden con el primer y último año de la niñez y adolescencia de los hijos.

Conforme se mencionó al comenzar el capítulo, las implicancias de este modelo alternativo son relevantes tanto para el diseño de políticas públicas como las comparaciones entre países, pues pone de relieve una fuente potencial de heterogeneidad inobservada previamente ignorada: la edad de los hijos en la que se mide el ingreso de los padres. Además, la importancia de considerar que el ingreso transitorio de los padres puede tener un efecto en las oportunidades futuras de los hijos, particularmente si ocurren durante su niñez y adolescencia, es mayor en países en desarrollo como Argentina y Chile que en los más desarrollados. En estos últimos países la mayor y más adecuada provisión pública de salud, educación y otros servicios sociales, así como el acceso al mercado de créditos y a oportunidades de empleo menos 
volátiles disminuyen la importancia de los componentes transitorios como permanentes del ingreso. En consecuencia, se espera que el sesgo que surge de excluir los ingresos transitorios del modelo intergeneracional estándar en las estimaciones de los niveles de persistencia del ingreso entre generaciones sea mayor en las sociedades menos desarrolladas.

\section{Método de estimación propuesto}

Nuevamente, la estimación de (18) plantea problemas en países en los que las bases de datos disponibles no incluyen información sobre el ingreso de los padres para los individuos encuestados, aunque contienen datos sobre otras características de los padres como su educación. Si bien, previamente se mencionó que una solución para estimar la EII en este caso es implementar el método $\mathrm{TSIV}^{87}$, esta técnica, por si sola, no sería adecuada para el modelo alternativo porque instrumenta el ingreso permanente pero no resuelve el problema de la exclusión del ingreso transitorio del modelo estimado. No obstante, bajo determinados supuestos el método TSIV ofrece una estimación apropiada de la elasticidad intergeneracional del ingreso permanente. Por tanto, teniendo en cuenta que la EII propuesta por este nuevo modelo está dada por (23) como la suma entre $\beta$ y las elasticidades correspondientes al componente transitorio del ingreso paterno, el estimador TSIV ofrece un límite inferior de B*. Para esto, conforme con el plim de este estimador dado en (21) es necesario que se cumplan los siguientes supuestos: (i) $\lambda_{2}=0$, teniendo en cuenta la ecuación (13) y si la educación se utiliza como variable instrumental, esto implica asumir que el efecto directo de la educación del padre sobre el ingreso del hijo es nulo, una vez que se controla por una medida de su ingreso a largo plazo, (ii) $\operatorname{Cov}\left(\hat{y}_{p}, u_{h a}\right)=0$ y (iii) $\lambda_{\alpha}=\mu_{\alpha}$ ó $\lambda_{\alpha}=\mu_{\alpha}=1$. En la sección 3 se discutió la factibilidad del cumplimiento de cada uno de estos supuestos.

Para derivar el límite superior de $\mathrm{B}^{*}$, en cambio, se propone una nueva técnica de estimación que combina algunas características del método TSIV y de un enfoque recientemente propuesto por Dang et al. (2014) para el análisis de la movilidad

\footnotetext{
${ }^{87}$ El método de IV tampoco sería adecuado por la misma razón.
} 
intrageneracional, concretamente de los movimientos dentro y fuera de la pobreza ${ }^{88}$. Bajo determinados supuestos, este método permitiría derivar un límite superior de la verdadera EII en el contexto del modelo alternativo antes descripto.

Supongamos que disponemos de dos ondas de una encuesta cross-section que son muestras aleatorias de la población subyacente de interés. Sea $X_{\text {pit }}$ un vector de características determinísticas o invariantes en el tiempo del i-ésimo padre observadas en la onda $\mathrm{t}=1,2$ como edad, sexo, educación, lugar de nacimiento, entonces la proyección lineal del ingreso observado del padre, en la onda t, $y_{p i t}$ en $X_{p i t}$ está dada por:

$$
y_{p i t}=\gamma_{t} X_{p i t}+\varepsilon_{p i t}
$$

Para los hijos de la muestra principal de la onda 2, sólo se dispone de información de características determinísticas o invariantes en el tiempo del i-ésimo padre pero no de su ingreso permanente ni transitorio cuando eran niños o adolescentes. Una forma de solucionar este problema es estimando este ingreso inobservado, utilizando una muestra alternativa, de un momento en el pasado en que los hijos de la muestra principal eran niños o adolescentes. En base a esta muestra secundaria (de la onda 1) es posible predecir el ingreso permanente de los padres en el pasado a partir de los parámetros estimados en esa muestra auxiliar y de las características observadas de los padres en la muestra principal (de la onda 2). La diferencia con el método TSIV antes mencionado es que también se utiliza la información de dos muestras para estimar el componente transitorio del ingreso $\left(\varepsilon_{\text {pit }}\right)$ en la niñez o adolescencia de los hijos de la muestra principal. Para esto, se deben realizar dos supuestos. El primero requiere que la muestra de la onda 1 y de la onda 2 sean de la misma población subyacente ${ }^{89}$. El segundo supuesto se refiere al comportamiento del término de error en (27),

\footnotetext{
${ }^{88}$ Cruces et al. $(2011,2015)$ realizaron un ejercicio de validación de este método para tres países de América Latina (Chile, Nicaragua y Perú), confirmando que los límites inferiores y superiores derivados con esta técnica funcionan como un intervalo de las verdaderas estimaciones de movilidad intrageneracional que podrían obtenerse con un conjunto de datos de panel. De igual forma, Martinez et al. (2013) implementaron, entre otros, el método de Dang et al. (2014) para evaluar distintos índices de movilidad intrageneracional.

${ }^{89}$ Según Dang et al. (2014), una forma de comprobar el cumplimiento de este supuesto en la práctica es examinando si las características invariantes en el tiempo de una cohorte cambian significativamente de una onda a otra de la encuesta utilizada para obtener la muestra auxiliar. El supuesto parece cumplirse para los datos de las encuestas de sección cruzada utilizadas en el ejercicio empírico de la sección 5.3.
} 
específicamente del componente que captura los shocks transitorios. Conforme con los supuestos realizados es posible derivar un límite superior de la EII definida en (23). Según postula el modelo alternativo, es razonable asumir que $\varepsilon_{p i 1}$ está correlacionado $\operatorname{con} \varepsilon_{p i 2}$. Existen dos razones por las que esto podría suceder. La primera, previamente analizada, es que los shocks en el ingreso pueden ser persistentes y estar positivamente correlacionados, siguiendo, por ejemplo, un proceso AR(1) como el planteado en (6). La segunda razón es que el término de error contenga un efecto fijo individual (Dang et al., 2014). Con el objetivo de ofrecer un límite superior para la EII (y por lo tanto inferior para la MII) se asume que los términos de error presentan una autocorrelación positiva perfecta $^{90}$. Es decir, $\delta=1$ y $\varepsilon_{p i 2}=\varepsilon_{p i 1}$. Los pasos para estimar la EII en este caso son:

$1^{\circ}$. Usando la muestra auxiliar de padres "representativos" de la onda 1, estimar por OLS una regresión de $y_{p i 1}^{1}$ en $X_{p i 1}^{1}$, donde el superíndice denota que estas son observaciones para los individuos observados en la onda 1 solamente. A partir de esta estimación obtener el estimador $\hat{\gamma}_{1}$ del vector de coeficientes $\gamma_{1}$. Luego, usando la muestra principal de padres de la onda 2, estimar una regresión de $y_{p i 2}^{2} \quad \mathrm{y} \quad X_{p i 2}^{2} \mathrm{y}$ obtener los residuos OLS:

$$
\varepsilon_{p i 2}^{2}=y_{p i 2}^{2}-\gamma_{2} X_{p i 2}^{2}
$$

$2^{\circ}$. Usar el estimador OLS $\hat{\gamma}_{1}$ de la onda 1 junto con los valores conocidos de $X_{p i 2}^{2}$ para estimar el ingreso de los padres en la onda 1, esto es, en algún año de la niñez o adolescencia de los hijos como sigue:

$$
\tilde{y}_{p i 1}^{2}=\hat{\gamma}_{1} X_{p i 1}^{2}+\varepsilon_{p i 2}^{2}
$$

$3^{\circ}$. Estimar la ecuación (29) para la mayor cantidad de años en la niñez o adolescencia de los hijos que sea posible, utilizando como ingreso de los padres en la niñez o adolescencia de sus hijos $\tilde{y}_{p i 1}^{2}$ en lugar del inobservado $y_{p i 1}^{2}$ y obtener un promedio de los ingresos estimados de los padres en distintos años de la niñez y adolescencia de sus hijos. Con este ingreso promedio como variable independiente,

\footnotetext{
${ }^{90}$ En realidad, Solon (1992) y Muller $(2008,2010)$, entre otros, asumen que $\delta=0.5$ en base a estimaciones previas. Por lo tanto, el supuesto de $\delta=1$ nos asegura la obtención de un límite superior para la EII, al menos, en este aspecto.
} 
obtener la EII estimada denotada por $\hat{B}_{L s}^{*}$ que constituye el límite superior de la verdadera B*. Bajo determinados supuestos (especificados en el Anexo B.6), el límite en probabilidad de este estimador ${ }^{91}$ está dado por:

$$
p \lim \hat{B}_{L S}^{*}=\frac{\beta \operatorname{Var}\left(y_{p}\right)+\lambda_{2} / T \operatorname{Var}\left(v_{p}\right)+\lambda_{2} / T \alpha \operatorname{Var}\left(\omega_{p}\right)+1 / T \operatorname{var}\left(\omega_{p q}\right) \sum_{q} \beta_{q} \Lambda_{q}+1 / T \sum_{k} \beta_{k} \operatorname{Var}\left(v_{p k}\right)}{\operatorname{Var}\left(y_{p}\right)+(1 / T) \operatorname{Var}\left(v_{p}\right)+(1 / T) \alpha \operatorname{Var}\left(\omega_{p}\right)}
$$

donde $\Lambda_{q}=\frac{1+\delta-\delta^{|q-r|+1}-\delta^{|q-m|+1}}{1-\delta}$ y $\alpha=1+2 \delta\left(\frac{T-\delta T-1+\delta^{T}}{T(1-\delta)^{2}}\right)$

De esta forma, dados los estimadores propuestos para el límite inferior y el límite superior y bajo los supuestos examinados, el método aquí presentado podría aportar una técnica para obtener un intervalo cerrado de la EII bajo el modelo alternativo $\left(\mathrm{B}^{*}\right)$.

\section{5. ¿Qué medida de ingreso utilizar?}

Otra cuestión a tener en cuenta en la estimación y comparación de la EII entre países es la elección del tipo de ingreso ${ }^{92}$ a utilizar, específicamente, ingresos laborales o ingresos familiares ${ }^{93}$. Conforme con la evidencia empírica, las estimaciones del nivel de persistencia intergeneracional varían con la clase de ingreso de los padres considerada. La principal razón para utilizar el ingreso laboral es que, conceptualmente, la capacidad de generar este tipo de ingresos (habilidades, esfuerzos, gustos o preferencias) no puede ser transferida de padres a hijos de la misma forma que una casa o un activo financiero. En este sentido, la movilidad con respecto a los ingresos laborales ofrece una mejor medida de las oportunidades sociales con relación al mérito individual. Además, desde una perspectiva teórica, los economistas no han analizado los efectos intergeneracionales del ingreso no laboral (Mazumder, 2005).

\footnotetext{
${ }^{91}$ En el Anexo B.6 pueden consultarse los detalles de la derivación del $p \lim \hat{B}_{L s}^{*}$.

${ }^{92}$ Una variable que mide mejor el bienestar económico que el ingreso y que sería más conveniente de utilizar en el análisis intergeneracional es el consumo, entre otras razones porque esta es la variable que típicamente se considera en la función de utilidad. Sin embargo, la falta de datos disponibles impiden considerar esta variable. Una de las excepciones es Mulligan (1997).

${ }^{93}$ También es importante controlar que los ingresos laborales o familiares incluyan los mismos conceptos y hayan sido medidos de forma relativamente similar en las bases de datos de ambos países, en este caso Chile y Argentina.
} 
Sin embargo, existen razones para preferir medidas alternativas del estatus económico de los padres cuando el objetivo es medir el efecto de los recursos familiares en las oportunidades económicas de los niños. En primer lugar, la utilización del ingreso familiar reduce el problema de sesgo de selección dentro del empleo que podrían presentar las estimaciones derivadas a partir del ingreso laboral, particularmente en el caso de las mujeres. Un hallazgo interesante en algunos estudios previos, que no ha sido suficientemente explicado, es que el ingreso familiar está más altamente asociado entre generaciones que el ingreso laboral (Behrman y Taubman, 1990; Solon, 1992; Peters, 1992; Mulligan, 1997; Coral y Heisz, 1999; Eide y Showalter, 1999; Shea, 2000; Altonji y Dunn, 2012). El ingreso familiar ofrece una medida más amplia de los recursos familiares que el ingreso laboral, implicando que la EII obtenida utilizando estos últimos ingresos estará subestimada (Corak, 2006). Por otra parte, si la movilidad depende de cuánto pueden invertir los padres en el capital humano de sus hijos, sería más adecuado y relevante concentrarse en el ingreso familiar total. Además, en el modelo tradicional de ingreso permanente, el deseo de suavizar el consumo a lo largo del tiempo sugiere que las familias usarían fuentes de ingresos no laborales para compensar shocks transitorios en los ingresos laborales. Por lo tanto, usar los ingresos familiares para estimar la EII constituye un enfoque alternativo para solucionar los problemas de medición asociados con las fluctuaciones transitorias en los ingresos laborales. Esto podría ser particularmente cierto en la parte inferior de la distribución del ingreso laboral de los padres, donde los individuos pueden percibir ingresos no laborales (como subsidios provenientes de planes sociales) en períodos en los que no perciben ingresos laborales (Mazumder, 2005). En síntesis, las mayores EII que se obtienen utilizando el ingreso familiar en comparación con las computadas considerando los salarios de los padres podría ser el resultado de varios factores: el efecto sobre el ingreso familiar de los ingresos de capital o de fuentes de ingresos no laborales, variaciones en la oferta laboral maternal y en el potencial de ingresos laborales, que afecta la distribución de ingresos familiares y el emparejamiento selectivo (o assortative mating) que es especialmente pronunciado entre las parejas con altos niveles educativos y puede incrementar las desigualdades entre las familias (Nolan et al., 2011). 
En definitiva, dado que existen razones para considerar el ingreso laboral como el ingreso familiar, en esta investigación se emplean ambos para estudiar y comparar los niveles de MII en Chile y Argentina, lo que permitirá, a su vez, examinar la sensibilidad de las conclusiones a las medidas de ingreso utilizadas.

\section{Datos}

La datos utilizados para examinar la MII en Chile provienen de la Encuesta Panel CASEN (EPCASEN) que nació en 2001 con el seguimiento ${ }^{94}$ de una muestra representativa de los hogares de las regiones III, VII, VIII y Metropolitana, a partir de la Encuesta de Caracterización Socieconómica Nacional (CASEN) 1996. El aporte que supone la onda 2006 de la encuesta es importante, en el contexto de Chile y de los países menos desarrollados, por el tamaño de su muestra y por el período de tiempo que abarca: diez años ${ }^{95}$. La población objetivo de la EPCASEN son todas las personas residentes en hogares privados de las de las regiones III, VII, VIII y Metropolitana, en múltiples momentos del tiempo, correspondiendo éstos a las ondas de recolección de los datos: 1996, 2001 y 2006. Un total de 20.942, 18.857 y 14.568 personas fueron encuestadas en 1996, 2001 y 2006 respectivamente. La muestra relevada en 1996 representa aproximadamente al $60 \%$ de la población total. El total de individuos seguidos entre 1996 y 2001 es de 15.038 y de 10.287 personas en las 3 ondas (19962001-2006). Esta disminución en el tamaño de la muestra inicial, se traduce en una tasa de atrición de 28,2\% entre 1996 y 2001, y de un 50,9\% entre 1996 y $2006^{96}$. La primera

\footnotetext{
${ }^{94}$ La encuesta busca seguir a los hogares iniciales de la encuesta, sin embargo, hay incorporación de individuos que no fueron encuestados en la primera onda. Esto se debe a nacimientos y a personas que no participaron en la nueva encuesta pero que se fueron a vivir con un hogar que si fue entrevistado. Por esto la encuesta distingue entre los MOM (miembros originales de la muestra) y los MTM (miembros temporales de la muestra). La encuesta sólo sigue a los MOM, siempre y cuando sigan viviendo en las regiones especificadas, sin importar si pasaron a vivir en un hogar distinto. De esta forma, si un MOM se traslada a vivir con un nuevo hogar o nuevas personas, estos nuevos miembros son seguidos como MTM y se siguen mientras el MOM viva con ellos, de la misma forma si a un hogar MOM llegan nuevos integrantes estos son seguidos como MTM y se siguen mientras vivan en el hogar MOM (Benezú et al., 2007).

${ }^{95}$ Según Yaqub (2000) sólo 5 de 44 países de bajo desarrollo humano y 7 de los 66 países de desarrollo humano intermedio cuentan con datos de este tipo de encuestas y ninguna de éstas cubre un período tan largo (Bendezú et al. 2007).

${ }^{96}$ Como estas tasas de atrición pueden considerarse elevadas y, principalmente, dado que la atrición afecta variables que resultan relevantes en esta investigación (como el ingreso) en las estimaciones se
} 
onda de la EPCASEN fue realizada entre noviembre y diciembre de 1996, la segunda, entre noviembre y diciembre de 2001, en tanto que, la última onda se realizó entre noviembre y diciembre de 2006 y enero y febrero de 2007. La encuesta contiene información sobre educación, ingresos, salud, participación en el mercado laboral, empleo, historia laboral y características de la vivienda (Benezú et al., 2007).

Con el objetivo de implementar la técnica TSIV así como el método propuesto en esta investigación, para evaluar su performance y realizar comparaciones con Argentina, se dividió el panel en dos sub-muestras, una correspondiente a la onda 1996 y otra, a la onda 2006, usando cada sub-muestra como dos encuestas cross-section repetidas. La encuesta de la onda 2006 del EPCASEN contiene información retrospectiva, para todos los jefes y cónyuges de hogar, sobre educación y ocupación de los padres. Esta muestra constituye, entonces, la denominada muestra principal de hijos del método TSIV. Por tanto, la muestra secundaria de padres "representativos" de los hijos de la muestra primaria se obtuvo de la onda 1996.

Otros datos empleados para Chile, a fin de realizar un análisis de robustez de las estimaciones obtenidas con el nuevo método aquí propuesto, se obtuvieron de las Encuestas CASEN para los años 1987, 1990, 1992, 1994, 1996, 1998, 2000 у 2006. Esta encuesta de hogares, con cobertura nacional (urbano-rural), regional (urbano-rural), provincial y a nivel comunal, cuya onda de 1996 sirvió de base para la EPCASEN 19962001-2006, contiene una gran variedad de información sobre características demográficas, laborales y socioeconómicas de la población. Las encuestas CASEN se relevan en noviembre y diciembre desde 1987, con periodicidad bianual o trianual, utilizando un muestreo estratificado, por conglomerados compacto (manzana de viviendas) y polietápico.

Los microdatos utilizados para Argentina provienen de distintas fuentes. Por una parte, la Encuesta Permanente de Hogares $(\mathrm{EPH})^{97}$ de los mismos años que los cubiertos por la EPCASEN. A diferencia de esta última, la EPH es una encuesta repetida de sección cruzada de los principales aglomerados urbanos del país que incluye información sobre ingresos y otras características sociodemográficas para padres e hijos

utilizaron las ponderaciones longitudinales corregidas por la probabilidad de volver a responder en la siguiente encuesta. Para detalles del método utilizado para corregir los factores de expansión por el problema de atrición, ver Bendezú et al. (2007).

${ }^{97}$ En el primer capítulo pueden consultarse las características y diseño de muestreo de la EPH. 
corresidentes. Esto permite la implementación de la técnica TSIV, de forma similar que la muestra seleccionada, con fines comparativos, de hijos y padres corresidentes del 2006 en la EPCASEN. A partir de la EPH del 2006 se obtiene la denominada "muestra principal de hijos y padres" y de la EPH de 1996 se deriva la "muestra secundaria o representativa".

Otras fuentes de datos utilizadas para Argentina provienen de tres encuestas de hogares con información retrospectiva sobre algunas características socioeconómicas de los padres (como su ocupación o su educación) para todos los individuos encuestados, independientemente de que éstos residen o no, al momento de la encuesta, en la misma vivienda que sus padres ${ }^{98}$. Esto permitió, después de implementar determinados criterios de armonización de las bases de datos, realizar comparaciones temporales de los niveles de MII. La primera encuesta de este tipo es la Encuesta de Desarrollo Social de 1997 (EDS). La EDS se realizó en octubre de 1997 en base a una muestra de alrededor de 26.000 viviendas urbanas de todo el país. El universo de la muestra abarca a la población residente en localidades de 5.000 o más habitantes, que representa el $96 \%$ de la población urbana del país y el $83,4 \%$ de la población total. Es decir, la muestra de la EDS de 1997 es aproximadamente representativa de la población nacional. La principal ventaja de esta encuesta es la disponibilidad de información sobre características ocupacionales de los padres de todos los adultos de 25 a 65 años entrevistados. En particular, la EDS 1997 incluye información sobre la situación de actividad, la categoría ocupacional y la calificación de la tarea de los padres cuando los hijos tenían alrededor de 15 años.

La segunda encuesta con información retrospectiva es la Encuesta de Percepción de Planes Sociales (EPPS). Este encuesta fue realizada entre mediados de junio y mediados de agosto de 2007 por Equipos MORI Argentina y el Centro de Estudios Distributivos, Laborales y Sociales de la Universidad Nacional de La Plata (CEDLAS) en el marco del proyecto "Política de Ingresos en Argentina" de la Unidad de Protección Social para América Latina y el Caribe del Banco Mundial. La muestra de la EPPS consiste de 2.500 jefes de hogar y cónyuges residentes en hogares particulares de todo

\footnotetext{
98 Esto elimina el problema de sesgo de selección por corresidencia potencialmente existente en las estimaciones de movilidad intergeneracional obtenidas a partir de muestras de padres e hijos que cohabitan en una misma vivienda, como por ejemplo, las provenientes de la EPH.
} 
el territorio de Argentina. Por lo tanto, la muestra es representativa de hogares a nivel nacional, regional y urbano-rural $^{99}$ (CEDLAS-MORI-BM, 2007). Por esto, en el ejercicio de comparación con Chile, la muestra secundaria, necesaria para implementar el método TSIV con los datos de la EPPS, es obtenida, en este caso, de la Encuesta de Desarrollo Social (EDS) relevada en octubre de 1997 es aproximadamente representativa de la población nacional. Entonces, la muestra secundaria obtenida a partir de esta encuesta es apropiada para la implementación del método TSIV utilizando como muestra principal los datos de la EPPS. Asimismo, en el contexto del presente estudio, la principal ventaja de la EPPS es la información que provee sobre la edad y educación de los padres para todos los encuestados. De forma similar, la EPCASEN, además de los datos sobre los padres que se derivan de la estructura longitudinal, incluye información retrospectiva sobre la educación y la ocupación de los padres, para los jefes y cónyuges de hogar encuestados. Además los años de realización de ambas encuestas están separados por un intervalo de 10 años, similar al período de tiempo cubierto por la EPCASEN. La EPPS permite también analizar la robustez de los resultados derivados con la $\mathrm{EPH}$.

La Encuesta del International Social Survey Programme (ISSP) 2009 realizada en Argentina entre julio y noviembre de 2010 por el CEDOP-UBA es otra de las bases de datos utilizadas (en adelante se hará referencia a esta encuesta como EISSP 2010). El ISSP es un programa de colaboración internacional que realiza encuestas anuales sobre diversos tópicos de las ciencias sociales desde 1985 en diversos países del mundo. El módulo implementado en la Encuesta ISSP 2009 (denominado Social Inequality IV) incluye, entre otras variables relevantes, información sobre la situación ocupacional y la profesión del padre y de la madre durante la adolescencia de todos los individuos adultos mayores de 18 años entrevistados. La muestra correspondiente a Argentina consiste de 1133 observaciones y es representativa de la población nacional. El diseño muestral es estratificado en tres etapas (unidades de área, hogares y personas) con un selección aleatoria en cada etapa (ISSP Research Group, 2012) ${ }^{100}$.

\footnotetext{
99 Esta es una gran ventaja respecto de la EPH que sólo es representativa de los centros urbanos más poblados del país.

${ }^{100}$ Debe señalarse que la muestra para Argentina de esta encuesta internacional no contaba con un factor de ponderación o expansión. Por tanto, a fin de obtener estimaciones comparables con las restantes encuestas utilizadas y que permitan hacer inferencias para toda la población se computaron los factores de
} 
Las tres encuestas mencionadas previamente se utilizan para examinar los cambios temporales en la MII en Argentina. Como estas encuestas no contienen información sobre el ingreso de los padres de cada hijo adulto de la muestra, a fin de predecir su ingreso en la adolescencia de los hijos o en un período lo más cercano posible a esta etapa, se emplea otra muestra anterior obtenida a partir de los datos de la EPH relevada en un año previo al de la muestra principal. Esta muestra secundaria, obtenida a partir de la EPH de 1992. Son varias las razones que justifican la elección de la EPH de este año para obtener la muestra secundaria de padres "representativos". En primer lugar, como uno de los objetivos de este artículo es examinar los cambios de la MII en el tiempo resulta conveniente emplear una misma muestra secundaria para predecir el ingreso paterno de los hijos en cada uno de los años considerados. La utilización de la EPH de 1992 para obtener la muestra de padres representativos implica que el ingreso paterno será estimado 18, 15 y 5 años atrás para los hijos observados en la EISSP 2009, la EPPS 2007 y la EDS 1997. Asimismo, la EPH de 1992 contiene información sobre características ocupacionales de los individuos entrevistados, particularmente las obtenidas del denominado Clasificador Nacional de Ocupaciones (CNO) de 1991, que permiten construir variables instrumentales para predecir el ingreso paterno, similares a las disponibles en la muestra principal. Además, 1992 es un año de relativa estabilidad macroeconómica a diferencia de años previos (1990 y 1991) para los que también se dispone de datos de la misma encuesta. Por otra parte, la EPH de 1992 cubre una mayor cantidad de aglomerados ${ }^{101}$ que las EPH de años anteriores. No obstante, más allá de las razones mencionadas que justifican la utilización de la EPH de 1992, se emplearon EPH de años anteriores a fin de realizar un análisis de la robustez de los resultados obtenidos al modificar la muestra secundaria.

Como la EPH recolecta información sólo de los centros urbanos más importantes del país en términos de tamaño de población, las muestras son representativas de las ciudades pero no de toda la población nacional. Esto podría llevar a una sobrestimación de la movilidad generacional en el país porque la muestra considerada no representa grupos de la población para quienes se espera que la persistencia generacional de

expansión correspondientes a partir de la información disponible en la EISSP 2010 relacionada con el diseño muestral.

${ }^{101}$ Para obtener una muestra anual de padres representativos a partir de la EPH, se unieron las observaciones correspondientes a los dos relevamientos realizados dentro del año en el caso de la EPH de 1992. Los aglomerados cubiertos por las EPH en 1992 son 27. 
ingresos sea mayor, tales como aquellos que viven en áreas rurales o pequeños centros urbanos. No obstante, en Argentina un elevado porcentaje de la población total habita en centros urbanos, y la muestra de la EPH, actualmente representa aproximadamente el $70 \%$ de la población urbana y el $60 \%$ de la población total del país. Además, como análisis de robustez de los resultados obtenidos se realizaron estimaciones utilizando una muestra secundaria obtenida a partir de la EDS de 1997 que tiene mayor cobertura geográfica.

En el Cuadro 1 y el Cuadro A1 del Anexo se observan las principales características de las muestras de padres e hijos con las que se desarrolla el análisis comparativo de los niveles de persistencia intergeneracional de ingresos entre Chile y Argentina. Como se observa en el Cuadro 1 para el análisis comparativo con Chile sólo se incluyeron en el análisis a los hijos varones, dados los problemas de sesgo de selección muestral normalmente asociados con la participación femenina en el mercado laboral. Por esto, sería riesgoso asignar una interpretación económica a las diferencias observadas entre las EII para las mujeres de Argentina y Chile porque la participación laboral femenina en Chile es inusualmente baja, al menos en el contexto latinoamericano. Conforme con las estadísticas obtenidas, la tasa de actividad de las hijas (en zonas urbanas) es del $72 \%$ en Chile y $77 \%$ en Argentina ${ }^{102}$. Como este problema de selección muestral es menor entre los varones, dadas sus mayores tasas de participación en el mercado laboral (87\%, en Chile y 88\%, en Argentina), se considera que las comparaciones de las EII estimadas para la muestra de padres e hijos varones son más apropiadas.

\footnotetext{
${ }^{102}$ Cabe advertir que, si bien la identificación de los ocupados es similar en la EPCASEN y en la EPH, la de los desocupados no lo es. Mientras en la primera encuesta, se considera desocupados a todos los que sin tener un trabajo lo buscaron en los últimos dos meses previos a la encuesta, en la EPH, el período de búsqueda considerado es el mes previo a la encuesta. Por lo tanto, es probable que de poder implementarse el mismo criterio de identificación de los desempleados usados en la EPH, en la muestra chilena, la tasa de desocupación y, por tanto, la de actividad resulten menores que las computadas. Aun así, sin este ajuste, la participación femenina estimada en la EPCASEN es menor que la observada en la Argentina.
} 
Cuadro 1. Características de las muestras de hijos varones y padres. Chile y Argentina.

\begin{tabular}{|c|c|c|c|c|}
\hline \multirow{3}{*}{ Variables } & \multicolumn{2}{|c|}{ Chile } & \multicolumn{2}{|c|}{ Argentina } \\
\hline & \multicolumn{4}{|c|}{ Hijos y padres corresidentes que habitan en áreas urbanas en 2006} \\
\hline & Hijos (25 a 58 años) & Padres (30 a 58 años) & Hijos (25 a 58 años) & Padres (30 a 58 años) \\
\hline \multicolumn{5}{|l|}{ Edad } \\
\hline Edad en 1996 & 22.258 & 46.154 & 22.800 & 47.204 \\
\hline Edad en 2006 & 32.271 & 55.971 & 32.800 & 57.204 \\
\hline \multicolumn{5}{|l|}{ Nivel educativo en 2006} \\
\hline Sin instrucción & 0.014 & 0.006 & 0.005 & 0.010 \\
\hline E. Básica Incompleta & 0.027 & 0.079 & 0.054 & 0.119 \\
\hline E. Básica Completa & 0.036 & 0.129 & 0.183 & 0.360 \\
\hline E. Media Incompleta & 0.201 & 0.350 & 0.169 & 0.160 \\
\hline E. Media Completa & 0.368 & 0.257 & 0.260 & 0.182 \\
\hline E. Superior Incompleta & 0.136 & 0.026 & 0.205 & 0.053 \\
\hline E. Superior Completa & 0.216 & 0.154 & 0.124 & 0.117 \\
\hline \multicolumn{5}{|l|}{ Situación ocupacional } \\
\hline Empleado en 1996 & 0.444 & 0.913 & - & - \\
\hline Empleado en 2006 & 0.768 & 0.841 & 0.782 & 0.750 \\
\hline Desempleado en 1996 & 0.063 & 0.014 & - & - \\
\hline Desempleado en 2006 & 0.097 & 0.019 & 0.102 & 0.065 \\
\hline Activo en 1996 & 0.507 & 0.927 & - & - \\
\hline Activo en 2006 & 0.866 & 0.860 & 0.884 & 0.815 \\
\hline \multicolumn{5}{|l|}{ Ingresos en 2006 (\$ corrientes) } \\
\hline Ingreso laboral total & 180766.4 & 260480.6 & 707.4 & 920.4 \\
\hline Ingreso de la ocupación principal & 235300.9 & 300979.9 & 679.4 & 873.1 \\
\hline Ingreso familiar total & 728656.8 & 753825.6 & 2373.8 & 2482.6 \\
\hline \multirow{2}{*}{\multicolumn{5}{|c|}{$\begin{array}{l}\text { Ingresos predichos en } 1996 \text { (en } \\
\text { \$ de 2006) }\end{array}$}} \\
\hline & & & & \\
\hline Ingreso laboral total & - & 250488.3 & - & 1385.1 \\
\hline Ingreso de la ocupación principal & - & 246105 & - & 1294.5 \\
\hline Ingreso familiar total & - & 461231.5 & - & 1888.7 \\
\hline Observaciones & 538 & 342 & 3633 & 2366 \\
\hline \multirow{2}{*}{ Variables } & \multicolumn{4}{|c|}{ Hijos jefes y cónyuges de hogar con información sobre sus padres } \\
\hline & Hijos de 25 a 58 años & Hijos de 30 a 45 años & Hijos de 25 a 58 años & Hijos de 30 a 45 años \\
\hline Edad & 43.151 & 38.089 & 42.288 & 37.537 \\
\hline Nivel educativo & & & & \\
\hline Sin instrucción & 0.003 & 0.002 & 0.004 & 0.005 \\
\hline E. Básica Incompleta & 0.069 & 0.056 & 0.065 & 0.033 \\
\hline E. Básica Completa & 0.033 & 0.020 & 0.248 & 0.236 \\
\hline E. Media Incompleta & 0.317 & 0.358 & 0.207 & 0.214 \\
\hline E. Media Completa & 0.280 & 0.332 & 0.261 & 0.253 \\
\hline E. Superior Incompleta & 0.084 & 0.046 & 0.084 & 0.114 \\
\hline E. Superior Completa & 0.214 & 0.186 & 0.130 & 0.146 \\
\hline \multicolumn{5}{|l|}{ Nivel educativo del padre } \\
\hline Sin instrucción & 0.142 & 0.176 & 0.081 & 0.098 \\
\hline Primaria Incompleta & 0.277 & 0.281 & 0.240 & 0.195 \\
\hline Primaria Completa & 0.200 & 0.201 & 0.399 & 0.442 \\
\hline Secundaria Incompleta & 0.133 & 0.139 & 0.077 & 0.074 \\
\hline Secundaria Completa & 0.188 & 0.153 & 0.154 & 0.147 \\
\hline Universitaria Incompleta & 0.016 & 0.021 & 0.014 & 0.018 \\
\hline Universitaria Completa & 0.044 & 0.029 & 0.036 & 0.026 \\
\hline $\begin{array}{l}\text { Ingresos familiares totales (\$ } \\
\text { corrientes) }\end{array}$ & 630794.6 & 579348.6 & 1930.76 & 1946.46 \\
\hline $\begin{array}{l}\text { Ingresos familiares totales } \\
\text { predichos de su padre } 10 \text { años } \\
\text { antes (en \$ año muestra ppal.) }\end{array}$ & 350555.7 & 449544.8 & 1937.92 & 2039.44 \\
\hline Observaciones & 1089 & 551 & 666 & 317 \\
\hline
\end{tabular}

Fuente: Elaboración propia sobre la base de EPCASEN y EPH.

Nota: los ingresos predichos de los padres se obtuvieron empleando como instrumento su nivel educativo. El promedio de los ingresos predichos laborales y de la ocupación principal de ambos padres es la media de la suma del ingreso laboral del padre y de la madre. El ingreso laboral total incluye los ingresos mensuales de la ocupación principal y los ingresos mensuales de las ocupaciones secundarias (sueldos o salarios, bonificaciones, gratificaciones y remuneraciones en especie). 
En el Cuadro 2 se resumen algunas estadísticas descriptivas de la muestra principal de hijos utilizadas para examinar los cambios temporales en los niveles de MII en Argentina. En este caso, se incluyen en el análisis a todos los hijos, varones y mujeres, a fin de contar con un mayor número de observaciones. No obstante, las estimaciones también se realizaron para varones y mujeres por separado. En este cuadro se puede observar la información sobre las características paternas disponibles en cada una de las encuestas: la calificación y la categoría ocupacional de los padres disponibles en la EDS 1997 y EISSP 2010 así como el nivel educativo paterno que surge de la EPPS 2007.

Cuadro 2. Características de la muestra de hijos de 25 a 64 años de 1997, 2007 y 2010. Argentina

\begin{tabular}{|c|c|c|c|}
\hline \multirow{2}{*}{ Características } & \multicolumn{3}{|c|}{$\begin{array}{c}\text { Hiijos de } 25 \text { a } 64 \text { con residentes en áreas } \\
\text { urbanas }\end{array}$} \\
\hline & EDS 1997 & EPPS 2007 & EISSP 2010 \\
\hline Edad & 42.41 & 43.30 & 43.07 \\
\hline \multicolumn{4}{|l|}{ Nivel educativo } \\
\hline Sin instrucción & 0.011 & 0.008 & 0.003 \\
\hline Primario Incompleto & 0.131 & 0.113 & 0.062 \\
\hline Primario Completo & 0.293 & 0.294 & 0.215 \\
\hline Secundario Incompleta & 0.180 & 0.191 & 0.222 \\
\hline Secundario Completo & 0.162 & 0.201 & 0.153 \\
\hline Superior Incompleto & 0.109 & 0.096 & 0.148 \\
\hline Superior Completo & 0.114 & 0.097 & 0.129 \\
\hline \multicolumn{4}{|l|}{ Nivel educativo del padre } \\
\hline Menor a secundario completo & - & 0.846 & - \\
\hline Secundario completo y más & - & 0.154 & - \\
\hline \multicolumn{4}{|l|}{ Calificación ocupacional del padre } \\
\hline Calificación técnica o profesional & 0.802 & - & 0.868 \\
\hline Sin calificación o calificación operativa & 0.198 & - & 0.132 \\
\hline \multicolumn{4}{|l|}{ Tipo de ocupación del padre } \\
\hline Tareas de producción, Auxiliares de Producción y de & & 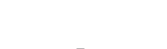 & \\
\hline Reparación de Bienes & 0.513 & - & 0.606 \\
\hline Tareas Administrativo-Contables & 0.078 & - & 0.135 \\
\hline Tareas de Comercialización & 0.123 & - & 0.077 \\
\hline Tareas de Transporte, Vigilancia y Seguridad y de Servicios & 0.287 & - & 0.183 \\
\hline Ingresos familiares per cápita (\$ constantes de dic. 2012 ) & 2464.37 & 1496.49 & 2156.56 \\
\hline $\begin{array}{l}\text { Ingresos familiares per cápita predichos de su padre (en \$ } \\
\text { constantes de dic 2012) }\end{array}$ & & & \\
\hline En base a su educación/calificación & 1226.32 & 1181.27 & 1159.59 \\
\hline En base a su ocupación & 1129.02 & - & 1181.99 \\
\hline Observaciones & 23560 & 1391 & 429 \\
\hline
\end{tabular}

Fuente: Elaboración propia sobre la base de EDS 1997, EPPS 2007 y EISSP 2010. 


\section{Resultados}

\subsection{Estimaciones del modelo intergeneracional del ingreso permanente: una primera comparación entre Argentina y Chile}

Las EII que se reportan en el Cuadro 3 y se analizan en este apartado fueron estimadas en base al modelo intergeneracional del ingreso permanente, siguiendo a la gran mayoría de la literatura teórica y empírica ${ }^{103}$.

Cuadro 3. EII estimadas entre hijos varones de 25 a 58 años y padres corresidentes en 2006. Chile y Argentina.

\begin{tabular}{|c|c|c|c|c|c|c|c|c|}
\hline \multirow{3}{*}{$\begin{array}{c}\text { Ingreso del } \\
\text { padre en } 1996 \\
\text { y del hijo en } \\
2006 \\
\end{array}$} & \multicolumn{7}{|c|}{ CHILE - Áreas urbanas } & \multirow{3}{*}{$\begin{array}{c}\text { ARGENTINA - } \\
\text { Áreas urbanas } \\
\text { EPH } 2006 \\
\text { TSIV }\end{array}$} \\
\hline & \multicolumn{6}{|c|}{ EPCASEN 1996-2001-2006 } & \multirow{2}{*}{$\begin{array}{c}\text { CASEN } 2006 \\
\text { TSIV }\end{array}$} & \\
\hline & OLS & & $\mathbf{T}$ & & & TSIV & & \\
\hline \multirow{4}{*}{$\begin{array}{l}\text { Ingreso laboral } \\
\text { total de ambos }\end{array}$} & \multirow{2}{*}{$\begin{array}{c}0.393 \\
(0.091)\end{array}$} & \multirow{2}{*}{\multicolumn{2}{|c|}{$\begin{array}{c}0.385 \\
(0.089)\end{array}$}} & \multirow{2}{*}{\multicolumn{2}{|c|}{$\begin{array}{c}0.862 \\
(0.213)\end{array}$}} & \multirow{2}{*}{$\begin{array}{c}0.493 \\
(0.218)\end{array}$} & 0.523 & \multirow{2}{*}{$\begin{array}{c}0.436 \\
(0.047)\end{array}$} \\
\hline & & & & & & & $(0.036)$ & \\
\hline & $0.215-0.571$ & 0.210 & 0.560 & 0.446 & 1.279 & $0.065-0.920$ & $0.452-0.595$ & $0.343-0.529$ \\
\hline & 185 & \multicolumn{2}{|c|}{185} & \multicolumn{2}{|c|}{185} & 185 & 2270 & 1065 \\
\hline \multirow{4}{*}{$\begin{array}{l}\text { Ingreso familiar } \\
\text { total }\end{array}$} & \multirow{2}{*}{$\begin{array}{c}0.389 \\
(0.089)\end{array}$} & \multirow{2}{*}{\multicolumn{2}{|c|}{$\begin{array}{c}0.602 \\
(0.078)\end{array}$}} & \multirow{2}{*}{\multicolumn{2}{|c|}{$\begin{array}{c}1.209 \\
(0.151)\end{array}$}} & \multirow{2}{*}{$\begin{array}{c}0.820 \\
(0.131)\end{array}$} & \multirow{2}{*}{$\begin{array}{c}0.803 \\
(0.035)\end{array}$} & \multirow{2}{*}{$\begin{array}{c}0.612 \\
(0.059)\end{array}$} \\
\hline & & & & & & & & \\
\hline & $0.215-0.564$ & $0.449-$ & 0.754 & 0.912 & 1.506 & $0.564-1.077$ & $0.734-0.871$ & $0.496-0.727$ \\
\hline & 348 & \multicolumn{2}{|c|}{348} & \multicolumn{2}{|c|}{348} & 348 & 3079 & 1473 \\
\hline Ingreso familiar & 0.452 & & 664 & & & 0.832 & 0.827 & 0.695 \\
\hline per cápita & $(0.106)$ & & $88)$ & & & (0.113) & $(0.035)$ & $(0.056)$ \\
\hline & $0.245-0.660$ & $0.491-$ & 0.837 & 1.050 & 1.617 & $0.611-1.054$ & $0.759-0.895$ & $0.585-0.805$ \\
\hline & 348 & & 48 & & & 348 & 3079 & 1473 \\
\hline Ingreso del & & & CHII & Áreas & anas & urales & & \\
\hline y del hijo en & & EPC & ASEN 1 & 96-2001 & 006 & & CASEN 2006 & \\
\hline 2006 & OLS & & $\mathbf{T}$ & & & TSIV & TSIV & \\
\hline Ingreso laboral & 0.469 & & 78 & & & 0.506 & 0.522 & \\
\hline total & $(0.084)$ & & $83)$ & & & $(0.168)$ & $(0.031)$ & \\
\hline & $0.304-0.633$ & $0.315-$ & 0.641 & 0.353 & 1.095 & $0.176-0.836$ & $0.461-0.583$ & \\
\hline & 247 & & 47 & & & 247 & 3606 & \\
\hline Ingreso familiar & 0.415 & & 501 & & & 0.748 & 0.793 & \\
\hline total & $(0.072)$ & & $60)$ & & & $(0.100)$ & $(0.029)$ & \\
\hline & $0.273-0.556$ & $0.483-$ & 0.719 & 0.764 & 1.214 & $0.552-0.945$ & $0.736-0.851$ & \\
\hline & 436 & & 36 & & & 292 & 4837 & \\
\hline Ingreso familiar & 0.461 & & 553 & & & 0.729 & 0.810 & \\
\hline per cápita & $(0.084)$ & & $66)$ & & & $(0.083)$ & $(0.027)$ & \\
\hline & $0.297-0.626$ & $0.524-$ & 0.783 & 0.872 & 1.324 & $0.567-0.892$ & $0.757-0.862$ & \\
\hline & 436 & & 36 & & & 436 & 4837 & \\
\hline
\end{tabular}

Fuente: Elaboración propia sobre la base de EPCASEN y EPH.

Nota: se reportan entre paréntesis los errores estándares computados con la técnica de bootstrap, debajo, el intervalo de confianza al 95\% y en la última fila de cada panel, el número de observaciones con el que se realizó cada estimación. Las EII fueron estimadas a partir de un modelo intergeneracional que incluye además del ingreso de hijos y padres (observado o predicho en base a su nivel educativo, según el método de estimación), controles cuadráticos para la edad de padres e hijos.

\footnotetext{
${ }^{103}$ Más adelante, en la sección 5.3, se examinarán las tasas de persistencia del ingreso entre generaciones a la luz del modelo alternativo sugerido por Muller (2010) que incorpora, además del efecto del componente permanente del ingreso, el impacto potencial de los shocks transitorios).
} 
En el caso de Chile, además de la técnica TSIV pueden implementarse los métodos OLS e IV, utilizando la información longitudinal de la EPCASEN. En cambio, en Argentina, si el ingreso de los padres intenta medirse en un momento de su ciclo vital lo más similar a aquel en el que se observa el ingreso de sus hijos, disminuyendo así el denominado life-cycle bias, ante la falta de datos de panel de largo alcance, debe recurrirse a una muestra auxiliar en el pasado, cuando los padres de la muestra principal eran más jóvenes. Es decir, debe implementarse el método TSIV. Para esto se utilizan los microdatos provenientes de la EPH del 2006 y de $1996^{104}$. Con fines comparativos, la muestra utilizada para ambos países está conformada por padres e hijos corresidentes en el 2006.

En base a las distintas estimaciones realizadas se decidió que el rango etario en el que se mide el ingreso de los hijos sea de 25 a 58 años y el de los padres, de 30 a 55 años. Se espera que esta selección permita minimizar el life-cycle bias, sin reducir demasiado el número de observaciones, en particular, las incluidas en la muestra de hijos de la EPCASEN.

Las EII estimadas por IV y por TSIV se realizaron utilizando como instrumento la educación del padre, concretamente su nivel educativo ${ }^{105}$. Para la implementación del método TSIV, en ambos países, se utilizó como muestra principal la de hijos y padres corresidentes en el 2006 y como muestra secundaria la conformada por hijos y padres "representativos" entrevistados en 1996 y de la misma cohorte de nacimiento que los incluidos en la muestra principal. La razón de la elección de este último año es que se corresponde al primer año de la EPCASEN. En otras palabras, la cobertura temporal del panel chileno impide medir el ingreso de los padres en años previos a 1996. Sin embargo, esto sería recomendable dado que, conforme surge de las características de la muestra de hijos y padres corresidentes, reportadas en el Cuadro 1, los padres, en 1996, año en que se mide su ingreso (o la correlación entre éste y su nivel educativo, en el caso del método IV o TSIV) tienen, en promedio, 10 años más que sus hijos en 2006.

\footnotetext{
${ }^{104}$ Estrictamente, la información disponible en la EPH permitiría estimar la EII por OLS e IV también para Argentina pero la muestra de hijos utilizada resultaría demasiado joven, incrementando considerable el denominado life-cycle bias. Para más detalles, ver sección 3.2.

${ }^{105}$ Se decidió instrumentar el ingreso de los padres utilizando dummies por nivel educativo porque, aunque la EPCASEN incluye suficiente información sobre los años de educación formal, en el caso de la EPH la variable está truncada en la cola superior, dado que no se consulta al encuestado por los años aprobados en el caso de la educación superior de grado (terciaria o universitaria) ni de postgrado finalizadas.
} 
Esto impide que el life-cycle bias pueda ser completamente eliminado. En efecto, conforme con la ecuación (20), el límite en probabilidad del estimador IV (y TSIV) está definido en función del cociente $\frac{\lambda_{\alpha}}{\mu_{\alpha}} \beta^{106}$ donde $\lambda_{\alpha}\left(\mu_{\alpha}\right)$ es el coeficiente de pendiente del ingreso permanente del hijo (padre) en el modelo para el ingreso del hijo (padre) observado a la edad $\alpha$, dado por las ecuaciones (17) y (18). Entonces, una forma de eliminarlo es midiendo en el ingreso de padres e hijos en la misma edad $\alpha$, de forma tal que $\lambda_{\alpha}=\mu_{\alpha}$. Esto implica, en términos empíricos, que el ingreso de los padres debería ser medido 20 años antes, en promedio, al momento en que se observa el ingreso de sus hijos. No obstante, como la edad promedio de los hijos (32 años, en la muestra chilena y 34 años, en Argentina y la de los padres (45 años, en Chile y 46 años, en Argentina) pertenece al intervalo en que, según las estimaciones de Haider y Solon (2006) y Böhlmark y Lindquist (2006), los coeficientes $\lambda_{\alpha}$ y $\mu_{\alpha}$ son más cercanos a uno.

Conforme con los límites en probabilidad derivados en la sección $3 \mathrm{y}$, en línea con los hallazgos encontrados por otros estudios (Solon, 1992; Zimmerman, 1992; entre otros), cuando se consideran las EII estimadas para la muestra chilena de hijos y padres varones, que habitan tanto en áreas urbanas como rurales, el estimador OLS parece subestimar la verdadera EII, en tanto que, el estimador IV tiende a sobrestimarla. En cambio, la EII obtenida con la técnica de TSIV toma, en muchos casos, valores intermedios al intervalo configurado por los estimadores anteriores. Por tanto, bajo determinados supuestos, podría estar más cerca de la verdadera EII que la computada con ellos.

Dados los problemas de sesgo de selección muestral normalmente asociados con la participación femenina en el mercado laboral y como este problema es menor entre los varones, por sus mayores tasas de participación en el mercado laboral $(87 \%$, en Chile y $88 \%$, en Argentina), se considera que las comparaciones de las EII estimadas con el método TSIV para la muestra de padres e hijos varones, que habitan en zonas urbanas, son más apropiadas. En general, las EII estimadas con el mismo método (TSIV) para Chile superan las obtenidas para Argentina, pero las diferencias no

\footnotetext{
${ }^{106}$ Asumiendo, para simplificar el análisis, que el segundo y tercer término convergen, en probabilidad, a cero.
} 
resultan, en su mayoría, estadísticamente significativas ${ }^{107}$. Cuando la variable utilizada es el ingreso laboral total ${ }^{108}$, las EII son de 0.49 en Chile y 0.44 en Argentina. Esta última aunque menor en 0.05 , no es estadísticamente diferente de la primera.

Las estimaciones de la EII para el ingreso laboral obtenidas para Chile, con la EPCASEN y la CASEN 2006, son similares a las reportadas por otros estudios empíricos que implementaron el método TSIV, utilizando la educación y la experiencia como instrumentos. Así, Núñez y Risco (2004) estiman, a partir de la Encuesta de Empleo y Desempleo del 2004, para el área de Gran Santiago, una elasticidad de 0.55, para una muestra de hijos de 23 a 35 años y de 0.58, para hijos de 23 a 55 años. En tanto que Núñez y Miranda $(2007,2011)$ obtienen una EII de 0.54, para hijos de 23 a 65 años, en base a la Encuesta de empleo y Desempleo del 2004 y de 0.71 para hijos de 23 a 40 años, utilizando la Encuesta CASEN del 2006.

Cuando la persistencia intergeneracional de ingresos se evalúa considerando el ingreso familiar total, nuevamente, la EII en Chile (0.8) resulta mayor, en 0.2 aproximadamente, a la estimada en Argentina (0.6), siendo esta diferencia significativa $^{109}$. Este hallazgo podría ser particularmente relevante teniendo en cuenta que el ingreso familiar ofrece una medida más amplia de los recursos familiares que el ingreso laboral y está menos sujeto a los problemas de medición asociados con las fluctuaciones transitorias en los ingresos laborales. De allí que, en línea con los resultados obtenidos por varios estudios empíricos ${ }^{110}$, se observa que, tanto en Chile como en Argentina el ingreso familiar está más altamente asociado entre generaciones que el ingreso laboral. Sin embargo, las limitaciones de las muestras con las que se obtuvieron estas EII para ambos países, concretamente, el potencial sesgo de selección por corresidencia presente en las estimaciones, impiden llegar a una conclusión

\footnotetext{
${ }^{107}$ Debe señalarse, no obstante, que, en algunos casos la magnitud de las diferencias puede considerarse económicamente significativa pero, como consecuencia de los elevados errores estándares asociados con las estimaciones obtenidas, particularmente los computados con las muestras obtenidas de la EPCASEN de menor tamaño, no resultan estadísticamente significativas.

${ }^{108} \mathrm{El}$ ingreso laboral total incluye los ingresos mensuales de la ocupación principal y los ingresos mensuales de las ocupaciones secundarias (sueldos o salarios, bonificaciones, gratificaciones y remuneraciones en especie)

${ }^{109}$ Un resultado similar se obtiene al considerar el ingreso familiar per cápita puesto que las EII estimadas para Chile utilizando esta medida de ingreso (0.872 y 0.760) también superan las obtenidas para Argentina (0.714 y 0.679), aunque en este caso las diferencias no son significativas, al menos al 5\%.

${ }_{110}$ Por ejemplo, Altonji y Dunn (2012), Shea (2000), Coral y Heisz (1999), Eide y Showalter (1999), Mulligan (1997), Solon (1992), Peters (1992), Behrman y Taubman (1990).
} 
definitiva porque no es posible determinar si el método implementado para corregir por este problema es efectivo.

Una forma de evaluar la robustez de ese resultado es utilizar una muestra alternativa que no presente este problema de selección. Una muestra de hijos que cumple con esta característica es la conformada por todos los jefes de hogar y cónyuges entrevistados en la EPCASEN de Chile, así como en la EPPS relevada en Argentina, a quienes se consultó por algunas características de sus padres. Concretamente, en ambas encuestas, se preguntó por el nivel educativo del padre ${ }^{111}$. Como la información sobre el ingreso de los padres sólo está disponible, en la EPCASEN para los hijos corresidentes y no lo está en el caso de la EPPS ${ }^{112}$, se implementó el método TSIV, utilizando como instrumento la educación del padre, reportada por todos los jefes de hogar y cónyuges encuestados. Asimismo, dado que la única variable de ingreso disponible en la EPPS es el ingreso familiar, las estimaciones se realizaron, para la muestra argentina, considerando esta variable, que, además, es de particular interés en este caso.

Las EII se estimaron para todos los jefes y cónyuges de hogar de dos grupos de edad, 25 a 58 años y 30 a 45 años, considerando rangos etarios similares para los padres "representativos" de la muestra auxiliar o secundaria. Esta última muestra fue obtenida de la onda de 1996 de la EPCASEN, en el caso de Chile y de la $\operatorname{EDS}^{113}$ de 1997, en el caso de Argentina.

Las EII reportadas en el Cuadro 4 confirman el resultado anterior, derivado a partir de las elasticidades computadas con la muestra de hijos y padres corresidentes (Cuadro 3). Esto es, que la persistencia intergeneracional del ingreso familiar parece ser mayor en Chile que en Argentina. Así, las elasticidades intergeneracionales del ingreso familiar total estimadas para hijos chilenos de 25 a 58 años así como para aquellos de 30 a 45 años (0.78) superan en 0.3 y 0.2 las computadas entre hijos argentinos del mismo rango etario (0.53 y 0.69). Estas pronunciadas diferencias resultan significativas, tanto desde un punto de vista económico como estadístico. Un resultado similar se obtiene al considerar como variable de resultado el ingreso familiar per cápita.

\footnotetext{
${ }^{111}$ Además, a diferencia de la EPPS, en la EPCASEN también se consulta por la educación de la madre. ${ }^{112}$ Las diferencias en el año en el que se obtuvo la muestra principal de hijos: 2006, EPCASEN y 2007, EPPS no se espera que afecte la comparabilidad de los resultados, entre otras razones porque la información de la onda 2006 de la EPCASEN se relevó entre noviembre y diciembre de 2006 y febrero y marzo de 2007.

${ }^{113}$ Ver sección 4, para más detalles sobre esta encuesta y las razones de su elección.
} 


\section{Cuadro 4. EII estimadas para hijos varones jefes y cónyuges de hogar. Chile y Argentina}

\begin{tabular}{|c|c|c|c|c|c|}
\hline País & Instrumento & Muestra & Ingreso & Hijos 25-58 años & Hijos 30-45 años \\
\hline \multirow{6}{*}{ Chile } & \multirow{6}{*}{$\begin{array}{l}\text { Educación } \\
\text { reportada por } \\
\text { los hijos en } \\
2006\end{array}$} & \multirow{6}{*}{$\begin{array}{c}\text { Hijos jefes y } \\
\text { cónyuges de } \\
\text { hogar en } 2006 \\
\text { (áreas urbanas y } \\
\text { rurales - } \\
\text { EPCASEN) }\end{array}$} & \multirow{2}{*}{$\begin{array}{l}\text { Ingreso laboral del padre } \\
\text { predicho en } 1996 \text { y del hijo } \\
\text { en } 2006\end{array}$} & $\begin{array}{c}0.776 \\
(0.107)\end{array}$ & $\begin{array}{c}0.779 \\
(0.160)\end{array}$ \\
\hline & & & & $\begin{array}{c}0.567-0.985 \\
978\end{array}$ & $0.465-{ }_{506}^{1.093}$ \\
\hline & & & \multirow{2}{*}{$\begin{array}{l}\text { Ingreso familiar total del } \\
\text { padre predicho en } 1996 \text { y } \\
\text { del hijo en } 2006\end{array}$} & $\begin{array}{c}0.804 \\
(0.077)\end{array}$ & $\begin{array}{c}0.919 \\
(0.109)\end{array}$ \\
\hline & & & & $\begin{array}{c}0.654-0.954 \\
1090\end{array}$ & $0.706-{ }_{548}^{1.132}$ \\
\hline & & & \multirow{2}{*}{$\begin{array}{l}\text { Ingreso familiar per cápita } \\
\text { del padre predicho en } 1996 \\
\text { y del hijo en } 2006\end{array}$} & $\begin{array}{c}0.804 \\
(0.072)\end{array}$ & $\begin{array}{c}0.855 \\
(0.100)\end{array}$ \\
\hline & & & & $\begin{array}{c}0.664-\quad 0.945 \\
1090\end{array}$ & $0.660-1.050$ \\
\hline \multirow{3}{*}{ Argentina } & \multirow{3}{*}{$\begin{array}{l}\text { Educación } \\
\text { reportada por } \\
\text { los hijos en } \\
2007\end{array}$} & \multirow{3}{*}{$\begin{array}{l}\text { Hijos jefes y } \\
\text { cónyuges de } \\
\text { hogar en } 2007 \\
\text { (áreas urbanas y } \\
\text { rurales - EPPS) }\end{array}$} & $\begin{array}{l}\text { Ingreso familiar total del } \\
\text { padre predicho en } 1997 \text { y } \\
\text { del hijo en } 2007\end{array}$ & $\begin{array}{c}0.530 \\
(0.079) \\
0.376-\quad 0.683 \\
552 \\
\end{array}$ & $\begin{array}{c}0.688 \\
(0.100) \\
0.491-\quad 0.885 \\
260\end{array}$ \\
\hline & & & \multirow{2}{*}{$\begin{array}{l}\text { Ingreso familiar per cápita } \\
\text { del padre predicho en } 1997 \\
\text { y del hijo en } 2007\end{array}$} & $\begin{array}{c}0.591 \\
(0.079)\end{array}$ & $\begin{array}{c}0.680 \\
(0.119)\end{array}$ \\
\hline & & & & $\begin{array}{c}0.435-0.746 \\
552\end{array}$ & $\begin{array}{c}0.447-0.913 \\
260\end{array}$ \\
\hline
\end{tabular}

Fuente: Elaboración propia sobre la base de EPCASEN, EPPS y EPH.

Nota: se reportan entre paréntesis los errores estándares computados con la técnica de bootstrap, debajo, el intervalo de confianza al 95\% y en la última fila de cada panel, el número de observaciones con el que se realizó cada estimación.

Por otra parte, cabe destacar algunos resultados metodológicamente relevantes. En primer lugar, dada la mayor cobertura geográfica de la EPPS, con relación a la EPH, que incluye, a diferencia de esta última, zonas rurales, podría esperarse que los niveles de persistencia intergeneracional del ingreso y, por tanto, las EII sean mayores que las reportadas en el Cuadro 3. En principio, se espera que los procesos de transmisión intergeneracional sean más fuertes en las poblaciones rurales donde las oportunidades de inversión en capital humano de los hijos así como sus posibilidades de acceder a mejores puestos laborales que los poseídos por sus padres son, en general, más limitadas. Sin embargo, cuando se comparan estas estimaciones para hijos y padres varones de 25 a 58 años, los resultados no muestran diferencias significativas entre ellas. Así, mientras las EII computadas con los datos de la EPPS son 0.5, para el ingreso familiar total y 0.6, para el familiar per cápita, las obtenidas a partir de la EPH son 0.6 y 0.69, respectivamente. Una explicación posible de este resultado podría estar relacionada con el bajo porcentaje de población total que habita en zonas rurales en Argentina (menos del 40\% según las estimaciones del último censo nacional de 
población del 2010). Este hallazgo sugiere, entonces, que los indicadores de movilidad intergeneracional obtenidos para Argentina, como la EII, utilizando la EPH son aproximadamente similares a lo que podrían derivarse a partir de una encuesta con cobertura geográfica urbana y rural.

Asimismo, el resultado anterior también sugeriría que las estimaciones realizadas con una muestra de padres e hijos que residen juntos (como la obtenida de la $\mathrm{EPH}$ ) no son estadísticamente diferentes a las estimadas a partir de una muestra de hijos con información retrospectiva de sus padres y sin sesgo de selección por corresidencia (como la derivada de la EPPS 2007). Una forma de apreciar el efecto aislado de este sesgo en las estimaciones es comparando las EII del Cuadros 3 y Cuadro 4 estimadas a partir de las muestras disponibles para Chile que presentan la misma cobertura geográfica e incluyen hijos del mismo grupo etario. Así, las EII obtenidas de la EPCASEN, para el ingreso familiar, a partir de una muestra de hijos y padres corresidentes son aproximadamente similares a las computadas con la muestra de hijos jefes y cónyuges de hogar, no sujetas a ese problema de sesgo de selección ${ }^{114}$. Así, las elasticidades intergeneracionales del ingreso familiar total y per cápita estimadas por TSIV para Chile, utilizando la muestra de hijos corresidentes con sus padres, son iguales a 0.79 y 0.81 , en tanto que, las obtenidas con la muestra de jefes y cónyuges de hogar (habiten o no con sus padres), del mismo rango etario y con el mismo método de estimación, son iguales a 0.8. Por tanto, estas EII no presentan diferencias estadísticamente significativas.

Además, las EII computadas para hijos varones de 30 a 45 años, resultan en general, mayores que las obtenidas con la muestra de 25 a 58 años. Aunque este resultado es esperable, dado que los hijos del primer grupo etario están, en promedio, en las edades en que, en teoría, el life cycle bias desaparece o es menor, las diferencias no son estadísticamente significativas. Este hallazgo confirma, entonces, que los resultados obtenidos con la muestra de hijos de 25 a 58 no llevan a conclusiones diferentes que las que podrían derivarse considerando la muestra de hijos en el primer rango etario.

\footnotetext{
${ }^{114}$ En cambio, la EII estimada con el ingreso laboral de los hijos son marcadamente menores cuando se utiliza la muestra con el potencial sesgo por corresidencia en comparación con la computada sin este sesgo y reportada en el Cuadro 4. Sin embargo, debe tenerse en cuenta que ésta última, a diferencia de la anterior, fue obtenida de una muestra conformada por jefes y cónyuges de hogar que tienen una edad promedio mayor (ver Cuadro 1). Este hecho, por tanto, podría también explicar, en parte, la diferencia observada entre las elasticidad intergeneracionales del ingreso laboral que se están comparando.
} 


\subsection{Cambios temporales en la persistencia intergeneracional del ingreso en Argentina}

En esta sección se analizan los cambios temporales que tuvieron lugar en los niveles promedio de inmovilidad del ingreso entre padres e hijos en Argentina. Con este fin se comparan las elasticidades intergeneracionales del ingreso familiar per cápita ${ }^{115}$ computadas a partir del modelo intergeneracional (3) ${ }^{116}$ y de la muestra de los hijos provenientes de la EDS 1997, la EPPS 2007 y la EISSP $2010^{117}$.

En primer lugar, a fin de comparar los niveles de persistencia intergeneracional del ingreso observados en 1997 y 2007 se utilizó como instrumento para predecir el ingreso paterno la educación de los padres reportada por los hijos. No obstante, como en la EDS 1997 no se consultó a los hijos con relación a la educación de sus padres, se usó la información sobre sus calificaciones ocupacionales aportada por todos los individuos entrevistados como proxy del nivel educativo paterno ${ }^{118}$.

Según los resultados de las primeras columnas del Cuadro 5, las EII estimadas en 1997 para hijos de 25 a 65 años jefes y cónyuges de hogar son significativamente más elevadas que las correspondientes a 2007, tanto para varones como mujeres. Así, mientras para el conjunto de hijos la EII asciende a 0.96 en 1997, es de 0.63 en 2007.

\footnotetext{
${ }^{115}$ Por razones de comparabilidad, sólo se consideró el ingreso familiar per cápita porque los datos de la EPPS 2007 no incluyen información sobre el ingreso laboral individual.

${ }^{116}$ Aunque sería interesante realizar también comparaciones temporales de las EII a partir del modelo intergeneracional alternativo, no es posible estimarlas con los datos de la ESSP 2007 ni de la EISSP 2010 porque no contienen información del ingreso del padre para los individuos entrevistados. Además, la EISSP 2010 tampoco ofrece un número adecuado de observaciones. Por esta misma razón, no se utilizó esta encuesta para obtener estimaciones comparables para Chile. Además, en este caso, por los motivos ya mencionados, sólo deberían incluirse en el análisis a los hijos varones pero esto reduciría el número de observaciones aún más.

${ }^{117}$ En este caso, no es posible realizar un análisis temporal por cohortes de nacimiento, similar al realizado en el Capítulo 1, que permitiría cubrir un período más extenso. Esto se debe a la imposibilidad de seguir a cada cohorte durante varios años y observar sus resultados a distintas edades a fin de poder distinguir los efectos de cohortes y edad.

${ }^{118} \mathrm{La}$ variable instrumental utilizada en este caso es una dummy igual a uno (cero) si el padre tenía, cuando el entrevistado era adolescente, una calificación técnica o profesional (calificación operativa o ninguna). Por tanto, se asume que esta variable dummy constituye una proxy de una dummy indicadora de si el nivel educativo del padre era igual o superior al nivel secundario completo. El coeficiente de esta última variable es el que se estima a partir de la muestra secundaria de padres "representativos" obtenida a partir de la EPH en 1992 para computar el ingreso predicho de los padres de la muestra principal de 1997. Se considera que la calificación ocupacional es una proxy razonable del nivel educativo dado que las estimaciones derivadas a partir de la muestra de la EPH de 1992 indican que la mayoría, más del 80\% de quienes tienen una calificación profesional o técnica presentan un nivel educativo igual o superior al secundario completo.
} 
Estos valores indican que la persistencia del ingreso entre padres e hijos medida a partir de este indicador se redujo un 34\% entre 1997 y 2007.

Cuadro 5. EII estimadas en 1997, 2007 y 2010 utilizando el método TSIV. Argentina.

\begin{tabular}{|c|c|c|c|c|c|c|c|c|c|}
\hline \multirow{2}{*}{ Año } & \multirow{2}{*}{$\begin{array}{c}\text { Base de } \\
\text { datos }\end{array}$} & \multirow{2}{*}{ Instrumento } & \multirow{2}{*}{ Muestra } & \multicolumn{6}{|c|}{ EII para hijos de 25 a 64 años } \\
\hline & & & & \multicolumn{2}{|c|}{ Todos } & \multicolumn{2}{|c|}{ Varones } & \multicolumn{2}{|c|}{ Mujeres } \\
\hline \multirow{10}{*}{1997} & \multirow{10}{*}{ EDS } & \multirow{3}{*}{\multicolumn{2}{|c|}{$\begin{array}{ll}\text { Educación del padre igual o Jefe y cónyuges de } \\
\text { superior al SC aproximada } & \text { hogar mayores de } 25 \\
\text { con su calificación } & \text { años }\end{array}$}} & \multicolumn{2}{|c|}{$\begin{array}{c}0.956 \\
(0.041)\end{array}$} & \multicolumn{2}{|c|}{$\begin{array}{c}1.093 \\
(0.067)\end{array}$} & \multicolumn{2}{|c|}{$\begin{array}{c}0.833 \\
(0.055)\end{array}$} \\
\hline & & & & $0.875-$ & 1.037 & $0.962-$ & 1.224 & $0.727-$ & 0.940 \\
\hline & & & & \multicolumn{2}{|c|}{6460} & \multicolumn{2}{|c|}{2929} & \multicolumn{2}{|c|}{3531} \\
\hline & & \multirow{3}{*}{\multicolumn{2}{|c|}{$\begin{array}{ll}\text { Educación del padre igual o } & \\
\text { superior al SC aproximada } & \text { Adultos de } 24 \text { a } 64 \\
\text { con su calificación } & \text { años }\end{array}$}} & \multicolumn{2}{|c|}{$\begin{array}{c}0.953 \\
(0.025)\end{array}$} & \multicolumn{2}{|c|}{$\begin{array}{c}1.038 \\
(0.039)\end{array}$} & \multicolumn{2}{|c|}{$\begin{array}{c}0.879 \\
(0.036)\end{array}$} \\
\hline & & & & $0.903-$ & 1.002 & $0.961-$ & 1.115 & $0.809-$ & 0.948 \\
\hline & & & & \multicolumn{2}{|c|}{18082} & \multicolumn{2}{|c|}{8390} & \multicolumn{2}{|c|}{9692} \\
\hline & & \multirow{4}{*}{$\begin{array}{l}\text { Características } \\
\text { ocupacionales del padre }\end{array}$} & \multirow{4}{*}{$\begin{array}{l}\text { Adultos de } 24 \text { a } 64 \\
\text { años }\end{array}$} & \multirow{2}{*}{\multicolumn{2}{|c|}{$\begin{array}{c}0.922 \\
(0.023)\end{array}$}} & \multirow{2}{*}{\multicolumn{2}{|c|}{$\begin{array}{c}0.977 \\
(0.031)\end{array}$}} & \multirow{2}{*}{\multicolumn{2}{|c|}{$\begin{array}{c}0.869 \\
(0.032)\end{array}$}} \\
\hline & & & & & & & & & \\
\hline & & & & $0.877-$ & 0.966 & $0.917-$ & 1.037 & $0.806-$ & 0.933 \\
\hline & & & & \multicolumn{2}{|c|}{17838} & \multicolumn{2}{|c|}{8270} & \multicolumn{2}{|c|}{9568} \\
\hline \multirow{2}{*}{2007} & \multirow{2}{*}{ EPPS } & Educación del padre igual o & Jefes y cónyuges de & & & & & & \\
\hline & & superior al SC & $\begin{array}{l}\text { hogar mayores de } 25 \\
\text { años }\end{array}$ & $0.465-$ & 0.805 & $0.260-$ & 0.793 & $0.455-$ & 0.916 \\
\hline & & Educación del padre igual o & Adultos de 24 a 64 & & 4) & & 9) & & \\
\hline & & con su calificación & años & $0.618-$ & 1.341 & $0.506-$ & 1.392 & $0.438-$ & 1.621 \\
\hline 2010 & EISSP & & & & & & & & \\
\hline & & Características & Adultos de 24 a 64 & & & & & & \\
\hline & & ocupacionales del padre & años & $0.543-$ & 0.995 & $0.463-$ & 1.063 & $0.473-$ & 1.107 \\
\hline & & & & & & & & & \\
\hline
\end{tabular}

Nota: SC: secundario completo. Los modelos intergeneracionales estimados incluyen como controles un polinomio cuadrático en la edad de los hijos y su sexo en el caso de la estimación para el total de la muestra. Entre las características ocupacionales se incluye la categoría ocupacional (trabajador independiente o asalariado), el tipo de ocupación y la calificación de la tarea desarrollada. Bajo de las EII se reportan los errores estándares computados con la técnica de bootstrap y a continuación, su intervalo de confianza al $95 \%$.

Fuente: Elaboración propia sobre la base de EDS 1997, EPPS 2007, EISSP 2010 y EPH 1992.

Además, cabe destacar el alto valor de la EII en 1997 para los hijos varones que asciende a 1.01 sugiriendo una persistencia de ingresos entre padres e hijos casi perfecta. Es decir, este coeficiente indica, en términos de desigualdad intergeneracional y siguiendo al interpretación que realiza Mulligan (1997) de la EII, que una diferencia de 1\% entre el ingreso de los padres en 1992 está asociada, manteniendo todo lo demás constante, con una diferencia de ingresos aproximadamente similar entre los hijos en 1997. En cambio, las EII estimada en 2007 para el conjunto de hijos varones es menos de la mitad de la computada para 1997. Debe señalarse nuevamente que la variable instrumental utilizada para estimar las EII en 1997 no es estrictamente igual a la empleada en 2007. Sin embargo, las EII estimadas para una muestra de hijos 
corresidentes $^{119}$ con sus padres, utilizando como variable instrumental para predecir el ingreso paterno la educación observada de los padres, son mayores a las computadas usando como instrumento su calificación ocupacional (ver Cuadro A2 del Anexo). Por tanto, estos resultados sugieren que en caso de utilizar la educación observada de los padres en lugar de su calificación como instrumento para predecir su ingreso, las EII estimadas en 1997 serían aún mayores que las reportadas en el Cuadro 5, confirmando las conclusiones anteriores.

Por otra parte, en el Cuadro 5 también se reportan EII comparables para 1997 y 2010 estimadas utilizando como variable instrumental las características ocupacionales de los padres (su categoría ocupacional, tipo de ocupación y calificación de la tarea desempeñada) para predecir su ingreso familiar per cápita ${ }^{120}$. Las EII así estimadas para hijos de 25 a 64 años son mayores en el primer año en comparación con el segundo. Esto indicaría, en principio, que los niveles promedio de movilidad de ingresos entre generaciones de 1997 eran menores que los estimados en 2010. No obstante, debe destacarse que las diferencias en las EII de 1997 y 2010 son considerablemente menores que las observadas entre 1997 y 2007. Así, para el total de hijos, la EII de 1997 es mayor en un 34\% a la estimada en 2007 pero sólo supera la de 2010 en un 17\%. Este resultado sugiere que parte de la mejora en la MII promedio observada en la década 1997-2007 parece haberse perdido entre este último año y 2010 ${ }^{121}$. Además, los elevados errores estándares de las EII estimadas en 2010, producto de la baja cantidad de observaciones con las que se computaron ${ }^{122}$, no permiten afirmar que las diferencias observadas con relación a las EII de 1997 son estadísticamente significativas. Asimismo, si se consideran las estimaciones obtenidas utilizando como variable instrumental la educación de los padres (aproximada con su calificación) para predecir

\footnotetext{
119 Sólo para esta muestra de hijos está disponible la información sobre la educación de sus padres que cohabitan con ellos en la EDS 1997.

${ }^{120}$ Cabe aclarar que si bien las bases de datos de la EDS 1997 y la EISSP 2010 tienen distintos clasificadores de las ocupaciones, se construyeron grupos ocupacionales para los padres estrictamente comparables.

${ }^{121}$ No obstante, las EII estimadas en estos dos últimos años no son estrictamente comparables porque no se obtuvieron a partir de una muestra similar de hijos. En la EPPS de 2007 la muestra está conformada por jefes y cónyuges de hogar, en cambio, en la EISSP de 2010 por adultos mayores de 18 años para quienes no hay información sobre su posición en el hogar.

${ }^{122}$ Como se especificó previamente, el tamaño de la muestra total disponible para Argentina en la EIISP 2010 es de 1133 observaciones.
} 
su ingreso familiar en 1992, las EII estimadas en 2010 para todos los hijos así como para las hijas de 25 a 64 años resultan levemente mayores que las computadas en 1997.

\subsection{Evaluación del nuevo modelo intergeneracional y su método de estimación}

En esta sección se evalúa el nuevo modelo intergeneracional formulado por Muller (2008). Conforme con éste, no sólo el ingreso permanente de los padres sino también sus ingresos transitorios pueden ser relevantes en la determinación del conjunto de oportunidades futuras de los hijos. A fin de obtener una estimación de la EII correspondiente a este modelo alternativo se estima, a partir del nuevo método de estimación propuesto, los límites inferiores y superiores de esta EII. La principal ventaja de este nuevo método es la posibilidad de estimar, utilizando encuestas cross-section repetidas, un intervalo de confianza de la movilidad o persistencia intergeneracional de ingresos que se derivaría en caso de disponer de datos de panel de largo alcance.

Las estimaciones reportadas en el Cuadro 6 se obtuvieron a partir de una muestra de hijos adultos que tenían entre 25 y 35 años en 2006, de forma tal que eran niños y jóvenes antes de 1996. Este rango etario elegido para los hijos responde a los argumentos de Muller (2008) relacionados con la relevancia de estimar la EII en la niñez o adolescencia de los hijos. La ampliación del rango etario de los hijos implica medir el ingreso de los padres fuera de la etapa vital considerada a priori relevante que es la de su niñez y adolescencia. En este punto resulta importante recordar que la hipótesis subyacente del modelo intergeneracional alternativo para considerar los shocks en el ingreso o los ingresos transitorios de los padres (además del ingreso permanente) es que se espera tengan un efecto en las oportunidades futuras de los hijos, particularmente si ocurren durante su niñez y adolescencia, posibilidad que es mayor en países en desarrollo como Argentina y Chile. Este supuesto está fundamentando por una variedad de evidencia empírica y el fuerte consenso que existe en la literatura acerca de que las experiencias de los primeros años de vida son fundamentales para determinar las oportunidades posteriores. Estos estudios analizan cómo el desarrollo en los primeros años y la adolescencia genera un proceso acumulativo de experiencias y factores que afecta los resultados futuros obtenidos por los hijos. Entre estos antecedentes se 
encuentra el de Cameron y Heckman (2001) que analizan el efecto del ingreso familiar sobre la educación de los hijos, encontrando resultados que sugieren que el ingreso familiar en las primeras etapas de la niñez y no luego, es el que afecta las probabilidades de asistencia a la universidad de los hijos ${ }^{123}$. Según Raczinsky (2006), la niñez temprana es una ventana de oportunidad para la adquisición de capacidad y aprendizaje que pueden lograrse a edades más avanzadas pero a un mayor costo. Sin embargo, algunos, como Heckman y Masterov (2007), entienden que hay habilidades cognitivas que sólo pueden alcanzarse al inicio de la vida y, por tanto, no hay modo de remediar las fallas en esa etapa. Esto tiene implicancias directas de políticas pues sugiere mover el foco de las políticas hacia la familia y la niñez temprana (Delorenzi et al. 2005). Además, según Cunha et al. (2006), las intervenciones a edad temprana promueven mejoras acumulativas -así como la falta de intervenciones producen desventajas que se acumulan -aumentando la productividad de la educación secundaria para los hijos de familias pobres que, en ausencia de restricciones crediticias, decidirían continuar sus estudios (FIEL, 2008).

Como se mencionó en la sección 3, el método TSIV permite, bajo determinados supuestos, estimar consistentemente la elasticidad intergeneracional del ingreso permanente. Dada la ecuación (21), el principal supuesto bajo el cual el estimador TSIV de la EII $(\beta)$ es consistente es que la educación del padre no tenga un efecto directo en el ingreso del hijo, después de controlar por el ingreso permanente del padre. Los estudios de Sewell y Hauser (1975), Corcoran et al. (1992) y Mazumder (2005) muestran que el efecto directo de la educación del padre en el ingreso del hijo no es estadísticamente distinto de cero, una vez que se controla por el ingreso del padre promediado durante varios años ${ }^{124}$. Si esto es así, el método TSIV permitiría obtener el límite inferior (LI) de la EII correspondiente al nuevo modelo intergeneracional puesto que sólo capturar el impacto del componente permanente del ingreso paterno.

\footnotetext{
${ }^{123}$ La explicación de este resultado está relacionada principalmente con la influencia acumulada del ingreso familiar desde el nacimiento hasta la adolescencia durante muchos años que afecta la habilidad y el capital humano general adquirido por el hijo antes de asistir a la universidad. Esto, a su vez, sugiere que el alcance de políticas educativas que promocionan la asistencia universitaria estará limitado por los efectos de largo plazo del ingreso familiar disponible durante la primera infancia, niñez y adolescencia.

${ }^{124}$ A partir de los datos de la EPCASEN también se obtuvo evidencia empírica a favor de este supuesto a partir de una regresión para el ingreso de los hijos que incluye entre las variables explicativas además de la educación del padre, un promedio del ingreso observado del padre durante los dos primeros años del panel. El coeficiente estimado de la educación del padre no resultó estadísticamente diferente de cero.
} 
Por otra parte, el límite superior (LS) de la EII se obtuvo implementando el nuevo método estimación propuesto en esta investigación. Con este objetivo, se utilizaron cinco muestras secundarias distintas a partir de los datos de la encuesta CASEN de Chile y de la EPH de Argentina de los años 1987 ${ }^{125}$, 1990, 1992, 1994 y 1996. Como los hijos adultos de la muestra principal del 2006 tienen entre 25 y 35 años, el ingreso predicho de los padres, a partir de su nivel educativo ${ }^{126}$, se computó en distintas etapas de su niñez y juventud. Así, por ejemplo, si la muestra secundaria es obtenida de la encuesta de 1987, el ingreso de los padres es estimado cuando los hijos tenían de 6 a 16 años, mientras que si se obtiene de la encuesta del año 1996, la estimación del ingreso paterno se realiza cuando los hijos tenían entre 15 y 25 años. Los ingresos paternos en cada uno de los cinco años mencionados se estiman implementando los diferentes pasos del método aquí propuesto para derivar el LS bajo el supuesto de que los términos de error están perfecta y positivamente correlacionados en el tiempo. Luego, los ingresos así estimados se promedian para obtener la EII correspondiente al LS.

Por otra parte, debe señalarse que las estimaciones se realizaron a partir una muestra de hijos que residen con su padre ${ }^{127}$. Si bien esto puede generar un sesgo en las estimaciones, de acuerdo con los resultados de la sección 5.1, éste parece no tener un efecto significativo sobre las EII. Además, no hay razones para suponer que la magnitud de este potencial sesgo sea distinta en las estimaciones obtenidas para Argentina en comparación con las derivadas para Chile utilizando el mismo método. Entonces, si el sesgo afecta las estimaciones de la misma forma, la diferencia entre ellas no lo incluiría. De esta forma, las comparaciones de las EII serían válidas.

\footnotetext{
${ }^{125}$ Aunque sería recomendable obtener la muestra de encuestas previas al año 1987, a fin de estimar el ingresos paterno durante la primera infancia de los hijos adultos de la muestra principal del 2006, no se dispone de una encuesta CASEN más antigua para Chile.

${ }^{126} \mathrm{Si}$ bien, de acuerdo con lo señalado por Dang et al. (2014) podría ser conveniente utilizar otras características paternas invariantes en el tiempo para predecir su ingreso como su lugar de residencia o sector de ocupación en años previos, no se dispone de esta información retrospectiva en las bases de datos utilizadas para Argentina. Además, se desconoce la posible dirección del sesgo presente en los estimadores de la EII $\left(\mathrm{B}^{*}\right)$ cuando se utilizan otras variables instrumentales diferentes al nivel educativo.

${ }^{127}$ La posible dirección de este sesgo no es clara a priori. Para más detalles, ver sección 3 del Capítulo 3. En Jiménez (2011a) se examinaron los métodos disponibles en la literatura para su corrección y se utilizó la técnica de dos etapas de Heckman. No obstante, no se consideró conveniente implementarlo aquí dados los supuestos restrictivos bajo los cuales este método solucionaría el problema y las limitaciones informativas de las bases de datos disponibles.
} 
En el Cuadro 6 se reportan los límites inferiores y superiores de la EII correspondientes al modelo alternativo aquí evaluado para Chile y Argentina, considerando como medida de ingreso de los hijos su ingreso laboral ${ }^{128}$ y como medida de ingreso de los padres su ingreso familiar total y per cápita ${ }^{129}$. Debe mencionarse también que para reducir el denominado life-cycle bias en las estimaciones se incluyeron a padres en edades centrales de 30 a 45 años.

\section{Cuadro 6. Límites de la EII del modelo alternativo. Hijos varones de 25 a 35 años} en 2006.

\begin{tabular}{|c|c|c|c|c|c|c|c|c|c|}
\hline \multirow{3}{*}{ Ingreso paterno } & \multicolumn{3}{|c|}{$\begin{array}{c}\text { CHILE (áreas urbanas y } \\
\text { rurales) }\end{array}$} & \multicolumn{3}{|c|}{ CHILE (áreas urbanas) } & \multicolumn{3}{|c|}{$\begin{array}{c}\text { ARGENTINA (áreas } \\
\text { urbanas) }\end{array}$} \\
\hline & $\mathbf{L I}$ & $\mathbf{L S}$ & Dif. $[2]-[1]$ & $\mathbf{L I}$ & $\mathbf{L S}$ & Dif. $[2]-[1]$ & LI & $\mathbf{L S}$ & Dif. $[2]-[1]$ \\
\hline & [1] & [2] & & [1] & [2] & & {$[1]$} & {$[2]$} & \\
\hline $\begin{array}{l}\text { Ingreso } \\
\text { familiar total }\end{array}$ & 0.493 & 0.599 & 0.106 & 0.519 & 0.594 & 0.075 & 0.466 & 0.606 & 0.140 \\
\hline Obs. & \multicolumn{2}{|c|}{1373} & & \multicolumn{2}{|c|}{905} & & \multicolumn{2}{|c|}{680} & \\
\hline Ingreso & & & & & & & & & \\
\hline $\begin{array}{l}\text { familiar per } \\
\text { capita }\end{array}$ & 0.467 & 0.555 & 0.088 & 0.490 & 0.550 & 0.060 & 0.412 & 0.547 & 0.135 \\
\hline Obs. & \multicolumn{2}{|c|}{1373} & & \multicolumn{2}{|c|}{905} & & \multicolumn{2}{|c|}{680} & \\
\hline
\end{tabular}

Nota: TSIV: estimador TSIV de la EII y LSMA: límite superior del modelo alternativo intergeneracional estimado a partir de cinco muestras secundarias. Los modelos intergeneracionales estimados tienen como variable dependiente el ingreso laboral de los hijos e incluyen como controles un polinomio cuadrático en la edad de los hijos.

Fuente: Elaboración propia sobre la base de EPCASEN 1996-2001-2006, CASEN 1987, 1992, 1994, 1996, 2006 y EPH 1987, 1992, 1994, 1996, 2006.

En todos los casos, el LS de la EII del nuevo modelo supera el LI, es decir, la estimación de la EII correspondiente al modelo tradicional de ingreso permanente tanto para el ingreso familiar total como para el ingreso familiar per cápita. Esto es consistente con la hipótesis de Muller (2008) sobre la importancia de considerar el ingreso transitorio de los padres, particularmente durante la niñez y adolescencia de los hijos. Además, las diferencias entre el LS y LI no son superiores a 0.11 en el caso de Chile y a 0.14 en el caso de Argentina. Teniendo en cuenta que LI no captura ningún efecto de los ingresos transitorios en tanto que el LS podría sobreestimar su efecto, este resultado sugiere que los ingresos transitorios de los padres explicarían como máximo el $18 \%$ y el $25 \%$ de la correlación del ingreso entre padres e hijos en Chile y Argentina,

\footnotetext{
${ }^{128}$ No se consideró como medida de resultado para los hijos su ingreso familiar porque al tratarse de muestras de padres e hijos corresidentes, la muestra de padres utilizada para estimar su ingreso transitorio incluiría en ese caso también a los hijos sesgando las estimaciones.

${ }^{129}$ No se utiliza el ingreso laboral del padre para evitar los problemas de sesgo de selección asociados con la participación laboral. Además, el ingreso familiar es una medida más adecuada de los recursos del hogar, para más detalles ver sección 3.5 .
} 
respectivamente. Según las estimaciones de Muller (2010), la contribución de los $\beta_{\mathrm{q}}$ (en 23) a la EII total es del 0.11 de manera que el $20 \%$ o más de la elasticidad intergeneracional en los Estados Unidos pueden responder al denominado ingreso transitorio de los padres.

Por otra parte, a partir de los límites estimados para la EII del modelo alternativo no se observan diferencias significativas en los niveles de persistencia intergeneracional del ingreso entre Chile y Argentina cuando se considera la correlación entre el ingreso laboral de los hijos varones y el ingreso familiar en su niñez y juventud.

\section{Conclusiones}

Los objetivos de este capítulo fueron varios. Por una parte, analizar el nivel así como los cambios temporales de la MII en Argentina. Las EII estimadas a partir de tres encuestas con información retrospectiva muestran un incremento significativo de la movilidad intergeneracional promedio entre 1997 y 2007 pero no así entre el primer año y 2010.

Asimismo, una forma de evaluar la magnitud del grado de persistencia intergeneracional del ingreso observado en Argentina es contrastándolo con el existente en otro país. Con este fin, se realizó un ejercicio de comparación con Chile a partir de estimaciones metodológicamente confrontables. Las características comunes y diferentes que presentan Argentina y Chile, susceptibles de tener un efecto importante en el proceso de transmisión intergeneracional, como sus niveles de crecimiento y desigualdad económica, hacen que este ejercicio empírico sea particularmente interesante. Así, una de las hipótesis que podrían evaluarse es si dado los comparativamente mayores niveles de desigualdad históricamente observados en Chile $^{130}$ y si la desigualdad y la movilidad están estrechamente relacionadas, siendo la primera un factor muy importante para la configuración de la segunda, más que, por ejemplo, los niveles de crecimiento económico, los niveles de persistencia intergeneracional son mayores en Chile o relativamente similares a los observados en Argentina. Los resultados obtenidos, a partir de las distintas técnicas de estimación

\footnotetext{
${ }^{130}$ Ver, por ejemplo, Núñez y Gutierrez (2004), Larrañaga (2002) y Ruiz-Tagle (1999).
} 
utilizadas para computar la EII, uno de los indicadores de inmovilidad o persistencia intergeneracional más utilizados en la literatura, sugieren que los niveles de movilidad intergeneracional son mayores en Chile que en Argentina cuando se considera como medida de ingreso el ingreso familiar total o per cápita. Así, las EII estimadas para hijos chilenos de 25 a 58 años (0.8) así como para aquellos de 30 a 45 años (0.9) superan en, aproximadamente, 0.2-0.3 las computadas entre hijos argentinos del mismo rango etario (0.5-0.6 y 0.7). Sin embargo, cuando se utiliza como medida de resultado económico para los hijos su ingreso laboral no se observan diferencias significativas en los niveles promedio de inmovilidad intergeneracional de ingresos de Chile y Argentina. Conforme a estimaciones comparables, la elasticidad intergeneracional del ingreso laboral asciende a 0.5 en Chile y 0.44 en Argentina.

Otro objetivo de esta investigación fue analizar formalmente las propiedades de los distintos métodos disponibles para estimar la EII que, como ya fue señalado, constituye uno de los indicadores de persistencia o inmovilidad de ingreso entre generaciones más utilizado en la literatura empírica. En este análisis, se destacaron las ventajas y se derivaron los sesgos potencialmente presentes en los distintos estimadores. Algunos de éstos fueron ignorados por la mayoría de los estudios disponibles. Asimismo, se evaluó empíricamente la performance de distintos estimadores de la EII, tanto los más utilizados en la literatura empírica como los obtenidos con un nuevo método aquí propuesto, utilizando uno de los paneles de datos más largo disponibles para un país de América Latina, como la Encuesta Panel CASEN 1996-2001-2006 (EPCASEN) de Chile. Los resultados obtenidos sugieren, en línea con los hallazgos de otros estudios empíricos (Solon, 1992, Zimmerman, 1992, entre otros), que las EII estimadas por OLS, utilizando un solo período para medir el ingreso paterno tienden a subestimar la verdadera, ocurriendo lo contrario con las estimaciones IV. Si esto es así, entonces el método TSIV presentaría una buena performance puesto que, en la mayoría de los casos, muestra valores intermedios, superiores a los computados con el método OLS pero inferiores a los obtenidos con la técnica IV. Esto es particularmente relevante puesto que el método TSIV permite computar y analizar los niveles de MII en países que, como Argentina, no disponen de datos longitudinales de largo alcance.

En el capítulo también se intentó evaluar empíricamente un modelo intergeneracional alternativo, recientemente propuesto por Muller (2010) y poco 
explorado en la literatura. La idea central de este enfoque es que, a diferencia de lo sostenido por el modelo intergeneracional tradicional, la transmisión relevante entre generaciones, no sólo tiene lugar en relación al componente permanente del ingreso sino también, a través de los ingresos transitorios. Este modelo tiene varias implicancias relevantes, como se mencionó previamente. Por un lado, sugiere que las comparaciones realizadas entre países en base al modelo estándar de transmisión intergeneracional del ingreso permanente, ignora una fuente potencial de heterogeneidad inobservada que podría afectar las estimaciones de la EII: la edad de los hijos en la que se mide el ingreso de sus padres. Por otra parte, las implicancias de política de este modelo alternativo son más inmediatas. Si el ingreso transitorio afecta el ingreso futuro de los niños, esto indicaría que el ingreso de los padres per se tiene un efecto en sus oportunidades, lo que generalmente es incompatible con las formulaciones incluso más débiles del enfoque de igualdad de oportunidades. Entonces, cuanto mayor sea la importancia del ingreso transitorio con relación al permanente en el proceso de transmisión intergeneracional, mayor será el campo para la intervención pública (Muller, 2008).

Como la estimación de este modelo alternativo, al igual que el tradicional, es particularmente problemática en países en los que las bases de datos disponibles no incluyen información sobre el ingreso de los padres para los individuos encuestados, en edades centrales del ciclo vital de ambos, se propuso en esta investigación un método novedoso de estimación. Este nuevo enfoque metodológico combina algunas características del método TSIV, para estimar el componente permanente del ingreso parental y del enfoque propuesto por Dang et al. (2014) que permite estimar su componente transitorio, particularmente durante la niñez y adolescencia de los hijos. Bajo determinados supuestos, con este método puede obtenerse un límite inferior y superior de la verdadera EII en el contexto del modelo alternativo. Las estimaciones obtenidas con de la implementación del nueva técnica indican que, los ingresos transitorios de los padres explicarían como máximo el 18\% y el 25\% de la correlación del ingreso observada entre padres e hijos en Chile y Argentina, respectivamente. En definitiva, considerando que las principales inversiones en el capital humano de los hijos tienen lugar en su niñez y adolescencia, y que, tanto los componentes permanentes como transitorios del ingreso son relevantes para determinar las oportunidades futuras de los hijos, esos resultados sugieren que, los niveles reales de movilidad 
intergeneracional del ingreso en ambos países son similares y relativamente bajos. No obstante, estos resultados deben ser interpretados con precaución dados los supuestos bajo los cuales fueron derivados. Sin embargo, más allá de las limitaciones del método propuesto se considera que éste puede constituir un primer aporte para una línea de investigación sobre formas alternativas de analizar la MII en países con fuentes de información limitada. En particular, se trata de una propuesta metodológica inicial para examinar un aspecto relevante pero poco explorado en la literatura empírica como el impacto de los componentes transitorios del ingreso paterno sobre los resultados económicos obtenidos por sus hijos. Entonces, queda relegado para una investigación futura el estudio de otras técnicas de estimación que permitan relajar los supuestos del enfoque aquí propuesto. 


\section{Referencias}

Aaronson, D. y Mazumder, B. (2008). "Intergenerational Economic Mobility in the United 1940 to 2000”, Journal of Human Resources, 43(1), 139-172.

Altonji, J. G. y Dunn, T. A. (2012). "Relationships among the family incomes and labor market outcomes of relatives", en S. W. Polachek y K. Tatsiramos (eds.), Research in Labor Economics, Volume 35 (35th Anniversary retrospective), Bingley, Emerald Group Publishing Limited, $761-802$.

Andersen, L. (2001). "Social mobility in Latin America: links with adolescent schooling”, Inter-American Development Bank, IADB Research Network Working Paper R-433.

Angrist, J. D. y Krueger, A. B. (1992). "The effect of age at school entry on educational attainment: an application of instrumental variables with moments from two samples", Journal of the American Statistical Association, 87, 328-36.

Angrist, J. D. y Pischke, J-S. (2009), Mostly harmless econometrics: An empiricist's companion. Princeton, New Jersey: Princeton University Press.

Arellano, M. y Meghir, C. (1992). "Female labour supply and on-the-job search: an empirical model estimated using complementary data set", The Review of Economic Studies, 59(3), 537-59.

Atkinson, A. B., Maynard, A. K. y Trinder, C. G. (1983). Parents and children: incomes in two generations. Londres: Heinemann Educational Books.

Azevedo, V. M. R. y Bouillon, C. P. (2010). "Intergenerational social mobility in Latin America: a review of existing evidence", Economic Analysis Review, 25(2), 7-42.

Baker, M., y Solon, G. (2003). "Earnings dynamics and inequality among Canadian men, 1976-1992: evidence from longitudinal tax records", Journal of Labor Economics, 21(2), 289-321.

Beccaria, L. A. (1978). "Una contribución al estudio de la movilidad social en la Argentina. Análisis de los resultados de una encuesta para el Gran Buenos Aires", Desarrollo Económico, 17(68), 593-618

Becker, G. (1987). Tratado sobre la familia. Madrid: Alianza Editorial.

Becker, G. S. y Tomes, N. (1979). “An equilibrium theory of the distribution of income and intergenerational mobility", Journal of Political Economy, 87(6), 1153-89. 
Behrman, J. R. y Taubman, P. (1985). "Intergenerational earnings mobility in the United States: some estimates and a test of Becker's intergenerational endowments model", The Review of Economics and Statistics, 67(1), 144-151.

Behrman, J. R. y Taubman, P. (1990). “The intergenerational correlation between children's adult earnings and their parents' income: Results from the Michigan Panel Survey of Income Dynamics, Review of Income and Wealth, 36(2), 115-27.

Bendezú, L., Denis, A., Sánchez, C., Ugalde, P. y Zubizarreta, J. R. (2007). "La Encuesta Panel CASEN: metodología y calidad de datos”. (Eds) J.R. Zubizarreta, Documento Metodológico No 1, Observatorio Social, Universidad Alberto Hurtado.

Bendezú, L., Denis, A., y Zubizarreta, J. R. (2007). “Análisis de atrición de la muestra en la Encuesta Panel CASEN", Observatorio Social, Universidad Alberto Hurtado.

Björklund, A. y Jäntti, M. (1997). "Intergenerational income mobility in Sweden compared to the United States", The American Economic Review, 87(5), 1009-18.

Björklund, A., Jäntti, M. y Nybom, M. (2012). "The role of parental income over the life cycle: a comparison of Sweden and the UK", Institute for the Study of Labor, IZA, Discussion Paper No. 7066.

Black, E. S. y Deveraux, P. J. (2011). "Recent developments in intergenerational mobility", En Ashenfelter, O. y Card, D., eds., Handbook of Labor Economics. Amsterdam: North-Holland Press.

Blanden, J, Goodman, A., Gregg, P. y Machin, S. (2002). "Changes in Intergenerational Mobility in Britain", Centre for Economic Performance, CEP Discussion Papers No. 0517, LSE.

Blanden, J. (2013). "Cross-country rankings in intergenerational mobility: A comparison of approaches in economics and sociolog," Journal of Economic Surveys, 27(1), 38-73.

Blanden, J., Gregg, P. y Machin, S. (2005). "Intergenerational in Europe and North America", A Report Supported by the Sutton Trust, Abril.

Böhlmark, A. y Lindquist, M. J. (2006). "Life-cycle variations in the association between current and lifetime income: replication and extension for Sweden", Journal of Labor Economics, 24(4), 876-896.

Brastberg, B., Roed, K., O. Raaum, R., Naylor, M., Jantti, T., Eriksson, y Osterbacka, E. (2007). "Nonlinearities in intergenerational earnings mobility: consequences for cross-country comparisons", The Economic Journal, 117(519), 72-92. 
Bratberg, E., Nilsen, O. A. y Vaage, K. (2005). "Intergenerational mobility: trends across the earnings distribution", Institute for the Study of Labor, IZA Discussion Papers No 1517.

Britain.' B.E. Journal of Economic Analysis and Policy 7(2): article 9.

Cameron, S. V. y Heckman, J. J. (2001). "The dynamics of educational attainment for black, hispanic and white males", Journal of Political Economy, 109(31), 455-499.

CEDLAS-MORI-BM (2007). "Manual y documentación metodológica de la Encuesta de Percepción de Planes Sociales". Documento técnico y base de datos correspondiente, Buenos Aires. Disponible en: www.cedlas.org/epps.

Celhay, P.; Sanhueza, C. y Zubizarreta, J.R. (2010). "Intergenerational mobility of income and schooling: Chile 1996-2006”, Revista de AnálisisEconómico, 25(2), 4363.

Chávez Molina, E. y Gutiérrez Ageitos, P. (2009). "Movilidad intergeneracional y marginalidad económica. Un estudio de caso en el Conurbano Bonaerense", Población de Buenos Aires, 6(10), 29-48.

Comi, S. (2004). "Intergenerational mobility in Europe: evidence from ECHP”, Centre for Household, Income, Labour and Demographic Economics, CHILD Working Papers No. 18.

Conconi, Adriana, Guillermo Cruces, Sergio Olivieri y Raúl Sánchez (2008). "E pur si muove? Movilidad, pobreza y desigualdad en América Latina”, Económica, La Plata, LIV(1-2), 21-159.

Corak, M. (2004). "Generational income mobility in North America and Europe: an introduction", en M. Corak, ed., Generational Income Mobility in North America and Europe. Cambridge: Cambridge University Press, 1-37 .

Corak, M. (2006). "Do poor children become poor adults? Lessons from a cross country comparison of generational earnings mobility", Research on Economic Inequality,13, $143-88$.

Corak, M. (2013). "Inequality from generation to generation: The United States in comparison", en R. Rycroft, ed., The Economics of Inequality, Poverty and Discrimination in the 21st Century, Santa Barbara: ABC-CLIO.

Corak, M. y Heisz, A. (1999). "The intergenerational earnings and income mobility of Canadian men: evidence from longitudinal income tax data", Journal of Human Resources, 34, 504-33. 
Corcoran, M.; Gordon, R., Laren, D. y Gary, S. (1992). "The association between men's economic status and their family and community origins," Journal of Human Resources, 27(3), 575-601.

Couch, K. A. y Lillard, D. R., (1998). "Sample selection rules and the intergenerational correlation of earnings", Labour Economics, 5(3), 313-29.

Couch, K. y Dunn, T. (1997). "Intergenerational correlations in labor market status: A comparison of the United State and Germany", Journal of Human Resources, 32(1). 210-232.

Couch, K. y Dunn, T. (1997). "Intergenerational correlations in labor market status: A comparison of the United State and Germany", Journal of Human Resources, 32(1), 210-232.

Cruces, G. (2008). “Tópicos de Economía distributiva”, Maestría en Economía, Facultad de Ciencias Económicas, Universidad Nacional de La Plata, La Plata, Material de clase no publicado.

Cruces, G., Lanjouw, P., Lucchetti, L., Perova, E., Vakis, R. y Viollaz, M. (2011). "Intragenerational mobility and repeated cross-sections. A three-country validation exercise”, World Bank Policy Research Working Paper No. 5916.

Cruces, G., Lanjouw, P., Lucchetti, L., Perova, E., Vakis, R. y Viollaz, M. (2015). "Estimating poverty transitions using repeated cross-sections: a three-country validation excercise”, The Journal of Economic Inequality, 13(2), 161-179.

Cunha, F., Heckman, J. J., Lochner, L. y Masterov, D. V. (2006). "Interpreting the evidence on life cycle skill formation", E. A. Hanusheck y F. Welch (eds.), Handbook of the Economics of Education, 1. Amsterdam: North Holland, 697812.

Dahan, M. y Gaviria, A. (2001). "Sibling correlations and intergenerational mobility in Latin America”, Economic Development y Cultural Change, 49(3), 537-54.

Dahl, M. y DeLeire, T. (2008). “The association between children's earnings and fathers' lifetime earnings: estimates using administrative data", Institute for Research on Poverty, Discussion Paper No. 1342-08.

Dang, Hai-Anh, Lanjouw, P., Luoto, J. y McKenzie, D. (2014). "Using repeated crosssections to explore movements into and out of poverty", Journal of Development Economics, 107, 112-128. 
Dearden, L., Machin, S. y Reed, H. (1997), "Intergenerational mobility in Britain", Economic Journal, 107(440), 47-64.

Delorenzi, S., Reed, J. y Robinson, P. (2005). Maintaining momentum. Promoting social mobility and life chances from early years to childhood, Institute for Public Policy Research (IPPR), Londres.

Dunn, C.E. (2007). “The intergenerational transmission of lifetime earnings: evidence from Brazil", The B.E. Journal of Economic Analysis and Policy, 7(2) (Contributions), 2, 1-40.

Eide, E. R. y Showalter, M. H. (1999). "Factors affecting the transmission of earnings across generations: a quantile regression approach", The Journal of Human Resources, 34(2), 253-67.

Ferreira, F. H. G., Messina, J., Rigolini, J., López-Calva, L. F., Lugo, M. A. y Vakis, R. (2013). Economic mobility and the rise of the Latin American middle class. Washington, DC: World Bank.

Ferreira, S. y Veloso, F. (2006). "Intergenerational mobility of wages in Brazil", Brazilian Review of Econometrics, 26(2), 182-211.

Fertig, A. R. (2001). "Trends in intergenerational earnings mobility in the U.S.” Journal of Income Distribution, 12, 108-30.

FIEL (2008). La igualdad de oportunidades en Argentina. Movilidad intergeneracional en los 2000, Buenos Aires: Temas grupo editorial.

Friedman, M. (1957). A theory of the consumption function, Princeton University Press, Princeton.

Gasparini L. y Cruces G. (2008). "Una distribución en movimiento: El caso de Argentina", Centro de Estudios Distributivos, Laborales y Sociales (CEDLAS), Universidad Nacional de La Plata, Documento de Trabajo del CEDLAS No.. 78.

Gasparini, L. y Lustig, N. (2011). "The rise and fall of income inequality in Latin America", Centro de Estudios Distributivos, Laborales y Sociales (CEDLAS), Universidad Nacional de La Plata, Documento de trabajo del CEDLAS No. 18.

Gasparini, L., Gutiérrez, F. y Tornarolli, L. (2007). "Growth and income poverty in Latin America and the Caribbean: Evidence from houshold surveys", Review of Income and Wealth, 53(2), 209-245.

Gaviria A. (2005). "Movilidad social en América Latina: realidades y percepciones", Mimeo, Universidad de Los Andes. 
Germani, G. (1963). "Movilidad social en la Argentina", En Movilidad social en la sociedad industrial, Lipset, M., S. y Bendix, R. (comps.), Buenos Aires: EUDEBA.

Goldberger, A. (1989), "Economic and mechanical models of intergenerational transmission", American Economic Review, 79(3), 504-513.

Grawe, N. D. (2004a). "Intergenerational mobility for whom? the experience of high and low earning sons in international perspective", M. Corak, ed., Generational Income Mobility in North America and Europe. Cambridge: Cambridge University Press.

Grawe, N. D. (2004b). "Reconsidering the use of nonlinearities in intergenerational earnings mobility as a test for credit constraints", Journal of Human Resources, 39(3), 813-27.

Grawe, N. D. (2006). "Lifecycle bias in estimates of intergenerational earnings persistence", Labour Economics, 13(5), 551-570.

Haider, S. y Solon, G. (2006). "Life-cycle variation in the association between current and lifetime earnings", The American Economic Review, 96(4), 1308-20.

Harding, S. J.; Jencks, Ch.; Lopoo, L. M. y Mayer, S. E. (2004). "The Changing Effect of Family Background on the Incomes of American Adults", en Bowles, S. Gintis, H. y Osborne, M. eds., Unequal Chances: Family Background and Economic Success, Princeton University Press and Russell Sage.

Heckman, J. (1979). "Sample selection as a specification error", Econometrica, 47, 153(61), 153-161.

Heckman, J. y Masteroz, D. (2007). "The productivity argument for investing in Young children", Allied Social Sciences Association, Chicago.

Hertz, T. (2005). "Attrition- and age-bias-corrected estimates of the trend in the intergenerational persistence of family income", documento no publicado, septiembre.

Hertz, T. (2007). "Trends in the intergenerational elasticity of family income in the United States", Industrial Relations: A Journal of Economy and Society, 46(1), 22 50 .

Hirvonen, L. (2008). "Intergenerational earnings mobility among daughters and sons: evidence from Sweden and a comparison with the United States", American Journal of Economics and Sociology, 67(5), 777-826. 
Holter, H. A. (2015). “Accounting for cross-country differences in intergenerational earnings persistence: the impact of taxation and public education expenditure", Quantitative Economics, 6(2), 385-428.

Hussain, M. A, Munk, M. D. y Bonke, J. (2008). “Intergenerational earnings mobilities - how sensitive are they to income measures?", Journal of Income Distribution An International Quarterly, 18(3-4), 79-92.

Jäntti, M., Bratsberg, B., Roed, K., Raaum, O., Naylor, R., Osterbacka, E., Bjorklund A., y Eriksson, T. (2006). "American exceptionalism in a new light: a comparison of intergenerational earnings mobility in the Nordic countries, the United Kingdom and the United States", The Institute for the Study of Labor, IZA Discussion Paper No. 1938.

Jenkins, S. (1987). "Snapshots versus movies: lifecycle biases and the estimation of intergenerational earnings inheritance," European Economic Review, 31(5), 11491158.

Jerrim, J., Choi, A. y Rodríquez, R. S. (2014). “Two-Sample Two-Stage Least Squares (TSTSLS) estimates of earnings mobility: how consistent are they?", Institute of Education, University of London, Department of Quantitative Social Science, Working Paper No. 14-17.

Jiménez, M. (2011a). "Un análisis empírico de las no linealidades en la movilidad intergeneracional del ingreso. El caso de Argentina", Centro de Estudios Distributivos y Laborales (CEDLAS), Universidad Nacional de La Plata, Documento de trabajo del CEDLAS No. 114.

Jiménez, M. (2011b). “¿Movilidad o persistencia intergeneracional del ingreso en la Argentina? Una aproximación empírica", Revista de Economía Política de Buenos Aires, Vols. 9 y 10, 91-141.

Jiménez, M. (2012). “Tendencias en la movilidad intergeneracional del ingreso. Un análisis desde el enfoque de igualdad de oportunidades para la Argentina”. Anales de la AAEP XLVII, Trelew.

Jiménez, M. (2016). "Movilidad intergeneracional del ingreso en Argentina. Un análisis de sus cambios temporales desde el enfoque de igualdad de oportunidades", Centro de Estudios Distributivos, Laborales y Sociales (CEDLAS), Universidad Nacional de La Plata, Documentos de trabajo de CEDLAS (enviado para publicar). 
Jorrat, J. R. (2000). Estratificación social y movilidad. Un estudio del área metropolitana de Buenos Aires. Tucumán: EUDET.

Jorrat, J. R. (2005). “Aspectos descriptivos de la movilidad Intergeneracional de clase en Argentina: 2003-2004", $7^{\circ}$ Congreso Nacional de Estudios del Trabajo, ASET.

Klevmarken, W. A. (1982). "Missing variables and two-stage least-squares estimation from more than one data set", en Proceeding of American Statistical Association, Business and Economic Statistics Section, 156-161.

Kuznets, S. S. (1966). Modern Economic Growth: Rate, Structure and Spread. New Haven: Yale University.

Labar, K. (2007). "Intergenerational Mobility in China", Centre d'Etudes et de Recherches sur le Développement International, CERDI Working Paper No. 29.

Larrañaga, O. (2002). “Distribución de ingresos en Chile, 1958 - 2001”, Departamento de Economía, Universidad de Chile, Documento de Trabajo No. 178.

Lee, Ch. y Solon, G. (2009). "Trends in Intergenerational Income Mobility", The Review of Economics and Statistics, 91(4), 766-772.

Lefranc, A. (2010). "The rise and fall of intergenerational earnings mobility in France over the last half century", Twenty-fourth conference and general assembly of the European Society for Population Economics, Essen.

Lefranc, A. y Trannoy, A. (2005). "Intergenerational earnings mobility in France: Is France more mobile than the US?", Annales d'Economie et de Statistique, 57-77.

Lefranc, A., Ojima, F. y Yoshida, T. (2010). "The intergenerational transmission of income and education: a comparison of Japan and France", J. Dronkers, ed., Quality and Inequality of Education, Springer, 229-253.

Leigh, A. (2007). "Intergenerational mobility in Australia", The B.E. Journal of Economic Analysis \& Policy, 7(2) (Contributions), Article 6.

Martinez, A.; Western, M.; Haynes, M. y Wojtek T. (2013). "Measuring income mobility using pseudo-panel data", The Philippine Statistician, 62(2), 71-99.

Mayer, S. E. y Lopoo, L. M. (2004). "What Do Trends in the Intergenerational Economic Mobility of Sons and Daughters in the United States Mean?" in Miles Corak, ed., Generational income mobility in North America and Europe. Cambridge: Cambridge University Press, 90-121.

Mayer, S. E. y Lopoo, L. M. (2005). "Has the intergenerational transmission of economic status changed?", Journal of Human Resources, 40(1), 169-85. 
Mazumder, B. (2001). "Earnings mobility in the US: A new look at intergenerational inequality", Federal Reserve Bank of Chicago working paper No. 2001-18.

Mazumder, B. (2005). "Fortunate sons: new estimates of intergenerational mobility in the United States using social security earnings data", The Review of Economics and Statistics, 87(2), 235-255.

Minicozzi, A. (2003). "Estimation of sons' intergenerational earnings mobility in the presence of censoring", Journal of Applied Econometrics, 18(3), 291-314.

Muller, S. M. (2008). "Begging the question: permanent income and social mobility", Economic Research Southern Africa, ERSA Working Paper No 75.

Muller, S. M. (2010). "Another problem in the estimation of intergenerational income mobility", Economics Letters, 108(3), 291-295.

Mulligan, C. B. (1997). Parental priorities and economic inequality. University of Chicago Press, Chicago.

Ng, I. YH, Shen, X. y Ho, K. W. (2009). "Intergenerational earnings mobility in Singapore and the United States", Journal of Asian Economics, 20(2), 110-119.

Nicoletti, C. (2008). "Multiple sample selection in the estimation of intergenerational occupational mobility", Institute for social and Economic Research (ISER). University of Essex, working paper No. 2008-20.

Nicoletti, C. y Ermisch, J. (2008). "Intergenerational earnings mobility: changes across cohorts in Britain”. B.E. Journal of Economic Analysis and Policy, 7(2), 1-36.

Nicoletti, C. y Francesconi, M. (2006). "Intergenerational mobility and sample selection in short panels", Journal of Applied Econometrics, 21(8), 1265-93.

Nolan, B., Esping-Andersen, G., Whelan, C. T., Maitre, B. y Wagnerb, S. (2011).’The role of social institutions in intergenerational mobility", T. M., Smeeding, R., Erikson y M., Jantii, eds., Persistence, privilege, and parenting: the comparative study of intergenerational mobility, New York: Russell Sage Foundation, 331-365.

Núñez, J. y Miranda, L. (2007). "Recent findings on intergenerational income and educational mobility in Chile", Department of Economics Universidad de Chile.

Núñez, J. y Miranda, L. (2011). "Intergenerational income and educational mobility in urban Chile", Estudios de Economía, 38(1), 195-221.

Núñez, J. y Risco, C. (2004). "Movilidad intergeneracional de ingresos en un país en desarrollo: el caso de Chile", Departamento de Economía, Universidad de Chile, Documento de trabajo No. 210. 
Núñez, J., y R. Gutiérrez. (2004). "Classism, meritocracy and discrimination in the Labor Market: The case of Chile", Departamento de Economía, Universidad de Chile, Documento de Trabajo No. 208.

Nybom, M. y Sthuler, J. (2016). "Heterogeneous income profiles and life-cycle bias in intergenerational mobility estimation”, The Journal of Human Resources, 51(1), 239268.

Österbacka, E. (2001). "Family background and economic status in Finland", Scandinavian Journal of Economics, 103, 467-484.

Osterberg, T. (2000). "Intergenerational income mobility in Sweden: what do tax-data show?", Review of Income and Wealth, 46(4), 421-36.

Pekkala, S. y Lucas, R. E. B. (2007) "Differences across cohorts in Finnish intergenerational income mobility", Industrial Relations, A Journal of Economic and Society, 46(1), 81-111.

Peters, E. (1992). "Patterns of intergenerational mobility in income and earnings", The Review of Economics and Statistics, 74(3), 456-66.

Quartulli, D. y Salvia, A. (2012). "La movilidad y la estratificación socio-ocupacional en la Argentina. Un análisis de las desigualdades de origen", Entramado y perspectivas. Revista de la carrera de Sociología, 2(2), 15-42.

Raaum, O., Bratsberg, B., Roed, K., Österbacka, E., Eriksson, T., Jäntti, M. y Naylor, R. (2007). "Marital sorting, household labor supply and intergenerational earnings mobility across countries", Institute for the Study of Labor, IZA Discussion Papers No. 3037.

Raczinsky, D. (2006). "Política de infancia temprana en Chile: condicionantes del desarrollo de los niños", Expansiva, En Foco, 77.

Rosen, H. S. (1985). Public Finance. Irwin: Homewood, IL.

Ruiz-Tagle, J. (1999). "Chile: 40 años de desigualdad de ingresos". Departamento de Economía, Universidad de Chile, Documentos de Trabajo No 165.

Sánchez Hugalde, A. (2004). "Movilidad intergeneracional de ingresos y educativa en España (1980-90)", Instituto de Economía de Barcelona, Document de treball No. 2004/1.

Sapelli, C. (2011)."Sudden stops in social mobility: intergenerational mobility in Chile", Instituto de Economía, Pontificia Universidad Católica de Chile, Documentos de Trabajo No. 400. 
Sewell, W. H. y Hauser, R. M. (1975). Education, occupation, and earnings: Achievement in the early career. New York: Academic Press.

Shea, J. (2000), “Does parents' money matter," Journal of Public Economics, 77(2), $155-184$.

Solon, G. (1989). "Intergenerational income mobility in the United States", Institute for Research on Poverty, University of Wisconsin, Discussion Paper No 894-89.

Solon, G. (1992). "Intergenerational income mobility in the United States", The American Economic Review, 82(3), 393-408.

Solon, G. (2002). "Cross-Country differences in intergenerational earnings mobility", Journal of Economic Perspectives, 16(3), 59-66.

Solon, G. (2004). "A model of intergenerational mobility variation over time and place", en Miles Corak, ed., Generational income mobility in North America and Europe. Cambridge: Cambridge University Press.

Stuhler, J. L. (2010). "Empirical strategies to eliminate life-cycle bias in the intergenerational elasticity of earnings literature", SOEP Papers on multidisciplinary panel data research No. 346.

Torche, F. (2005). "Social mobility in Chile in comparative perspective", American Sociological Review, 70(3), 422-450.

Vella, F. (1998). "Estimating models with sample selection bias: A survey", Journal of Human Resources, 33(1), 127-69.

Vogel, T. (2006). "Reassessing intergenerational mobility in Germany and the United States: The impact of differences in lifecycle earnings patterns", School of Business and Economics, Humboldt University, SFB 649 Discussion Papers No 055.

Yaqub, S. (2000). "Intertemporal welfare dynamics", Human Development Report Office (HDRO), United Nations Development Programme (UNDP), Human Development Occasional Papers (1992-2007).

Zimmerman, D. (1992). "Regression toward mediocrity in economic stature", The American Economic Review, 82(3), 409-29. 


\section{Anexo A}

Cuadro A1. Característica de la muestra de hijos y padres corresidentes en áreas urbanas y rurales. Chile, 2006

\begin{tabular}{|c|c|c|}
\hline Variables & Hijos (25 a 58 años) & Padres (30 a 58 años) \\
\hline \multicolumn{3}{|l|}{$\overline{\text { Edad }}$} \\
\hline Edad en 1996 & 22.413 & 46.242 \\
\hline Edad en 2006 & 32.419 & 56.040 \\
\hline \multicolumn{3}{|l|}{ Nivel educativo en 2006} \\
\hline Sin instrucción & 0.017 & 0.011 \\
\hline E. Básica Incompleta & 0.036 & 0.112 \\
\hline E. Básica Completa & 0.041 & 0.132 \\
\hline E. Media Incompleta & 0.226 & 0.342 \\
\hline E. Media Completa & 0.360 & 0.233 \\
\hline E. Superior Incompleta & 0.124 & 0.023 \\
\hline E. Superior Completa & 0.196 & 0.147 \\
\hline \multicolumn{3}{|l|}{ Situación ocupacional } \\
\hline Empleado en 1996 & 0.467 & 0.920 \\
\hline Empleado en 2006 & 0.787 & 0.843 \\
\hline Desempleado en 1996 & 0.065 & 0.013 \\
\hline Desempleado en 2006 & 0.090 & 0.017 \\
\hline Activo en 1996 & 0.532 & 0.933 \\
\hline Activo en 2006 & 0.876 & 0.860 \\
\hline \multicolumn{3}{|l|}{ Ingresos en 2006 (\$ corrientes) } \\
\hline Ingreso laboral total & 174037.1 & 252044.8 \\
\hline Ingreso de la ocupación principal & 218591.3 & 284807.7 \\
\hline Ingreso familiar total & 696647.6 & 726620.3 \\
\hline \multicolumn{3}{|l|}{ Ingresos predichos en 1996 (en } \\
\hline \multicolumn{3}{|l|}{ \$ de 2006) } \\
\hline Ingreso laboral total & - & 229116.6 \\
\hline Ingreso de la ocupación principal & - & 226071.6 \\
\hline Ingreso familiar total & - & 434418.4 \\
\hline Observaciones & 668 & 411 \\
\hline
\end{tabular}

Fuente: Elaboración propia sobre la base de EPCASEN y EPH.

Cuadro A2. EII estimadas en 1997 para hijos corresidentes con sus padres utilizando el método TSIV. Argentina

\begin{tabular}{|c|c|c|c|c|c|c|}
\hline \multirow{2}{*}{ Año } & \multirow{2}{*}{$\begin{array}{c}\text { Base de } \\
\text { datos }\end{array}$} & \multirow{2}{*}{ Muestra } & \multirow{2}{*}{ Instrumento } & \multicolumn{3}{|c|}{ EII para hijos de 25 a 64 años } \\
\hline & & & & Todos & Varones & Mujeres \\
\hline \multirow{8}{*}{1997} & \multirow{8}{*}{ EDS } & \multirow{8}{*}{$\begin{array}{c}\text { Hijos } \\
\text { corresidentes } \\
\text { con sus padres }\end{array}$} & Educación del padre & 0.954 & 0.996 & 0.918 \\
\hline & & & igual o superior al SC & $(0.086)$ & $(0.116)$ & $(0.136)$ \\
\hline & & & aproximada con su & $0.785-1.124$ & $0.768-1.223$ & $0.652-1.183$ \\
\hline & & & calificación ocupacional & 1622 & 831 & 791 \\
\hline & & & & 1.482 & 1.504 & 1.451 \\
\hline & & & Educación del padre & $(0.085)$ & $(0.104)$ & $(0.138)$ \\
\hline & & & igual o superior al SC & $1.316-1.648$ & $1.299-1.709$ & $1.180-1.722$ \\
\hline & & & & 1622 & 831 & 791 \\
\hline
\end{tabular}

Fuente: Elaboración propia sobre la base de EDS 1997. 


\section{Anexo B}

\section{B.1. Límite en probabilidad del estimador OLS sin life-cycle bias}

Dado el modelo (1), (2), (3) se tiene:

$$
\begin{aligned}
\operatorname{plim} \hat{\beta}_{O L S} & =\frac{\operatorname{Cov}\left(y_{p s}, y_{h s}\right)}{\operatorname{var}\left(y_{p s}\right)}=\frac{\operatorname{Cov}\left(y_{p}+u_{p s}, y_{h}+u_{h t}\right)}{\operatorname{var}\left(y_{p}+u_{p s}\right)} \\
\operatorname{plim} \beta_{O L S} & =\frac{\beta \operatorname{var}\left(y_{p}\right)+\operatorname{Cov}\left(y_{p}, u_{h t}\right)+\operatorname{Cov}\left(y_{h}, u_{p s}\right)+\operatorname{Cov}\left(u_{p s}, u_{h t}\right)}{\operatorname{var}\left(y_{p}\right)+\operatorname{var}\left(u_{p s}\right)+2 \operatorname{Cov}\left(y_{p}, u_{p s}\right)}
\end{aligned}
$$

Suponiendo que $\operatorname{Cov}\left(y_{p}, u_{h t}\right)=\operatorname{Cov}\left(y_{h}, u_{p s}\right)=\operatorname{Cov}\left(u_{p s}, u_{h t}\right)=0$, la estimación por OLS estará sujeta al tradicional sesgo de atenuación por error de medición en las variables. En particular, teniendo en cuenta que $u_{p i s}=\omega_{p i s}+v_{p i s}$, si se asume que $\omega_{\text {pis }}$ es también un componente de ruido blanco independiente del ingreso permanente, se tiene:

$$
\operatorname{plim} \hat{\beta}_{O L S}=\beta\left(\frac{\operatorname{var}\left(y_{p}\right)}{\operatorname{var}\left(y_{p}\right)+\operatorname{var}\left(\omega_{p s}\right)+\operatorname{var}\left(v_{p s}\right)}\right)
$$

Esta es la ecuación (4) de la sección 3.1.

\section{B.2. Límite en probabilidad del estimador PT sin life-cycle bias}

Dado el modelo (1), (2), (3) y utilizando $y_{p} \approx \frac{\sum_{t} y_{p t}}{T}$ se tiene:

$$
\operatorname{plim} \hat{\beta}_{P T}=\frac{\operatorname{Cov}\left(\frac{\sum_{t} y_{p t}}{T}, y_{h s}\right)}{\operatorname{var}\left(\frac{\sum_{t} y_{p t}}{T}\right)}=\frac{\operatorname{Cov}\left(\frac{1}{T} \sum_{t}\left(y_{p}+u_{p t}\right), y_{h}+u_{h t}\right)}{\frac{1}{T^{2}} \operatorname{var}\left(\sum_{t}\left(y_{p}+v_{p t}+\omega_{p t}\right)\right)}
$$


$\operatorname{plim} \hat{\beta}_{P T}=\frac{\operatorname{Cov}\left(\frac{1}{T} T y_{p}+\frac{1}{T} \sum_{t} u_{p t}, y_{h}+u_{h t}\right)}{\frac{1}{T^{2}} \operatorname{var}\left(T y_{p}+\sum_{t}\left(v_{p t}+\omega_{p t}\right)\right)}$

$\operatorname{plim} \hat{\beta}_{P T}=\frac{\operatorname{Cov}\left(y_{p}+\frac{1}{T} \sum_{t} u_{p t}, y_{h}+u_{h t}\right)}{\frac{1}{T^{2}}\left[T^{2} \operatorname{var}\left(y_{p}\right)+\sum_{t} \operatorname{var}\left(v_{p t}\right)+\sum_{t} \operatorname{var}\left(\omega_{p t}\right)\right]}$

$\operatorname{plim} \beta_{P T}=\frac{\beta \operatorname{var}\left(y_{p}\right)+\operatorname{Cov}\left(y_{p}, \frac{1}{T} \sum_{t} u_{p t}\right)+\operatorname{Cov}\left(y_{h}, u_{p s}\right)+\operatorname{Cov}\left(\frac{1}{T} \sum_{t} u_{p t}, u_{h t}\right)}{\operatorname{var}\left(y_{p}\right)+\frac{T}{T^{2}} \operatorname{var}\left(v_{p t}\right)+\frac{T}{T^{2}} \operatorname{var}\left(\omega_{p t}\right)}$

Asumiendo que $\operatorname{Cov}\left(y_{p}, \frac{1}{T} \sum_{t} u_{p t}\right)=\operatorname{Cov}\left(y_{h}, u_{p s}\right)=\operatorname{Cov}\left(\frac{1}{T} \sum_{t} u_{p t}, u_{h t}\right)=0$, se tiene:

$$
\operatorname{plim} \beta_{P T}=\beta \frac{\operatorname{var}\left(y_{p}\right)}{\operatorname{var}\left(y_{p}\right)+\frac{1}{T} \operatorname{var}\left(v_{p t}\right)+\frac{1}{T} \operatorname{var}\left(\omega_{p t}\right)}
$$

Esta es la ecuación (5) de la sección 3.1.

En el caso en que el término $\omega_{p t}$ que captura el efecto de shock transitorio en el ingreso siga un proceso AR(1) como el de la ecuación (6), entonces a partir de (B.3) y teniendo en cuenta la derivación de Mazumder (2001) se tiene:

$$
\operatorname{plim} \beta_{P T}=\beta \frac{\operatorname{var}\left(y_{p}\right)}{\operatorname{var}\left(y_{p}\right)+\frac{1}{T} \operatorname{var}\left(v_{p t}\right)+\frac{1}{T} \alpha \operatorname{var}\left(\omega_{p t}\right)}
$$

donde $\alpha=1+2 \delta\left(\frac{T-\delta T-1+\delta^{T}}{T(1-\delta)^{2}}\right)$

Esta es la ecuación (7) de la sección 3.1.

\section{B.3. Límite en probabilidad del estimador IV sin life-cycle bias}

Dado el modelo (1), (2) y (3), el límite en probabilidad del estimador IV, utilizando la educación de los padres como instrumento de su ingreso permanente es el siguiente: 


$$
\begin{aligned}
& p \lim \hat{\beta}_{I V}=\frac{\operatorname{Cov}\left(E_{p}, y_{h t}\right)}{\operatorname{Cov}\left(E_{p}, y_{p s}\right)} \\
& p \lim \hat{\beta}_{I V}=\frac{\operatorname{Cov}\left(E_{p}, \delta_{1} y_{p}+\delta_{2} E_{p}+\varepsilon_{h}+u_{h t}\right)}{\operatorname{Cov}\left(E_{p}, y_{p s}\right)}
\end{aligned}
$$

Si se asume que $\operatorname{Cov}\left(E_{p}, \varepsilon_{h}\right)=0$ y reemplazando $y_{p i}=y_{p i s}-u_{p i s}$, entonces:

$$
p \lim \hat{\beta}_{I V}=\frac{\operatorname{Cov}\left(E_{p}, \delta_{1} y_{p s}-\delta_{1} u_{p s}+\delta_{2} E_{p}+u_{h t}\right)}{\operatorname{Cov}\left(E_{p}, y_{p s}\right)}
$$

$$
p \lim \beta_{I V}=\frac{\operatorname{Cov}\left(E_{p}, \delta_{1} y_{p s}+\delta_{2} E_{p}\right)}{\operatorname{Cov}\left(E_{p}, y_{p s}\right)}+\frac{\operatorname{Cov}\left(E_{p}, u_{h t}\right)-\delta_{1} \operatorname{Cov}\left(E_{p}, u_{p s}\right)}{\operatorname{Cov}\left(E_{p}, y_{p s}\right)}
$$

$$
p \lim \beta_{I V}=\delta_{1}+\delta_{2} \frac{\operatorname{Var}\left(E_{p}\right)}{\operatorname{Cov}\left(E_{p}, y_{p s}\right)}+\frac{\operatorname{Cov}\left(E_{p}, u_{h t}\right)-\delta_{1} \operatorname{Cov}\left(E_{p}, u_{p s}\right)}{\operatorname{Cov}\left(E_{p}, y_{p s}\right)}
$$

Considerando la relación entre el parámetro de interés $\beta$ en (3) y los parámetros $\delta_{1}$ y $\delta_{2}$ en $(9)^{131}$, se tiene:

$$
\beta=\frac{\operatorname{Cov}\left(y_{s}, y_{p}\right)}{\operatorname{Var}\left(y_{p}\right)}=\frac{\operatorname{Cov}\left(\delta_{1} y_{p}+\delta_{2} E_{p}+\varepsilon_{h}, y_{p}\right)}{\operatorname{Var}\left(y_{p}\right)}=\delta_{1}+\delta_{2} \frac{\operatorname{Cov}\left(E_{p}, y_{p}\right)}{\operatorname{Var}\left(y_{p}\right)}
$$

Reemplazando $\delta_{1}$ a partir de (B.7) en (B.6), se obtiene:

$$
p \lim \hat{\beta}_{I V}=\beta+\delta_{2}\left[\frac{\operatorname{Var}\left(E_{p}\right)}{\operatorname{Cov}\left(E_{p}, y_{p s}\right)}-\frac{\operatorname{Cov}\left(E_{p}, y_{p s}\right)}{\operatorname{Var}\left(y_{p}\right)}\right]+\frac{\operatorname{Cov}\left(E_{p}, u_{h t}\right)}{\operatorname{Cov}\left(E_{p}, y_{p s}\right)}-\delta_{1} \frac{\operatorname{Cov}\left(E_{p}, u_{p s}\right)}{\operatorname{Cov}\left(E_{p}, y_{p s}\right)}
$$

Suponiendo que $\operatorname{Cov}\left(E_{p}, u_{p s}\right)=0$ y teniendo en cuenta que $\sigma_{E_{p}}=\sqrt{\operatorname{Var}\left(E_{p}\right)}$ y $\sigma_{y_{p}}=\sqrt{\operatorname{Var}\left(y_{p}\right)}$ se tiene:

$$
p \lim \beta_{I V}=\beta+\delta_{2} \sigma_{E_{p}}\left[\frac{1-\operatorname{Corr}\left(E_{p}, y_{p}\right)^{2}}{\operatorname{Corr}\left(E_{p}, y_{p}\right) \sigma_{y_{p}}}\right]+\frac{\operatorname{Cov}\left(E_{p}, u_{h t}\right)}{\operatorname{Cov}\left(E_{p}, y_{p s}\right)}
$$

Esta es la ecuación (10) de la sección 3.1.

\footnotetext{
${ }^{131}$ Este resultado se deriva a partir de la fórmula para variables omitidas.
} 


\section{B.4. Límite en probabilidad del estimador TSIV o TS2SLS sin life-cycle bias}

Dado el modelo definido en las ecuaciones (1), (2), (3), (11) y (14), el límite en probabilidad del estimador TSIV, utilizando la educación de los padres como instrumento de su ingreso permanente es:

$\operatorname{plim} \hat{\beta}_{T S 2 S L S}=\frac{\operatorname{Cov}\left(\hat{y}_{p}, y_{h}\right)}{\operatorname{Cov}\left(\hat{y}_{p}, y_{p}\right)}$

$\operatorname{plim} \hat{\beta}_{T S 2 S L S}=\frac{\operatorname{Cov}\left(\hat{y}_{p}, \lambda_{1} y_{p}+\lambda_{2} \hat{y}_{p}+u_{h}\right)}{\operatorname{Cov}\left(\hat{y}_{p}, y_{p}\right)}$

$\operatorname{plim} \hat{\beta}_{T S 2 S L S}=\frac{\lambda_{1} \operatorname{Cov}\left(\hat{y}_{p}, y_{p}\right)+\lambda_{2} \operatorname{Cov}\left(\hat{y}_{p}, \hat{y}_{p}\right)+\operatorname{Cov}\left(\hat{y}_{p}, u_{h}\right)}{\operatorname{Cov}\left(\hat{y}_{p}, y_{p}\right)}$

Suponiendo que $\operatorname{Cov}\left(\hat{y}_{p}, u_{h}\right)=0$, entonces

$\operatorname{plim} \hat{\beta}_{T S 2 S L S}=\lambda_{1}+\lambda_{2} \frac{\operatorname{Var}\left(\hat{y}_{p}\right)}{\operatorname{Cov}\left(\hat{y}_{p}, y_{p}\right)}$

Multiplicando y dividiendo el denominador por $\sigma_{\hat{y}_{p}} \sigma_{y_{p}}=\sqrt{\operatorname{Var}\left(\hat{y}_{p}\right)} \sqrt{\operatorname{Var}\left(y_{p}\right)}$ se tiene:

$\operatorname{plim} \beta_{T S 2 S L S}=\lambda_{1}+\lambda_{2} \frac{\sigma_{\hat{y}_{p}}}{\operatorname{Corr}\left(\hat{y}_{p}, y_{p}\right) \sigma_{y_{p}}}$

Dada la relación entre $\beta \mathrm{y}$ los parámetros $\lambda_{1} \mathrm{y} \lambda_{2}$ de la ecuación (14):

$$
\beta=\frac{\operatorname{Cov}\left(y_{s}, y_{p}\right)}{\operatorname{Var}\left(y_{p}\right)}=\frac{\operatorname{Cov}\left(\lambda_{1} y_{p}+\lambda_{2} \hat{y}_{p}+u_{h i}, y_{p}\right)}{\operatorname{Var}\left(y_{p}\right)}=\lambda_{1}+\lambda_{2} \frac{\operatorname{Cov}\left(\hat{y}_{p}, y_{p}\right)}{\operatorname{Var}\left(y_{p}\right)}
$$

Y reemplazando $\lambda_{1}$ según (B.10) en (B.9), se obtiene:

$$
\begin{array}{r}
\operatorname{plim} \hat{\beta}_{T S 2 S L S}=\beta+\lambda_{2}\left(\frac{\sigma_{\hat{y}_{p}}}{\operatorname{Corr}\left(\hat{y}_{p}, y_{p}\right) \sigma_{y_{p}}}-\frac{\operatorname{Cov}\left(\hat{y}_{p}, y_{p}\right)}{\sigma_{y_{p}}^{2}}\right) \\
\operatorname{plim} \hat{\beta}_{T S 2 S L S}=\beta+\lambda_{2}\left(\frac{\sigma_{\hat{y}_{p}}-\operatorname{Corr}\left(\hat{y}_{p}, y_{p}\right) \operatorname{Cov}\left(\hat{y}_{p}, y_{p}\right) / \sigma_{y_{p}}}{\operatorname{Corr}\left(\hat{y}_{p}, y_{p}\right) \sigma_{y_{p}}}\right) \\
\operatorname{plim} \beta_{T S 2 S L S}=\beta+\lambda_{2} \sigma_{\hat{y}_{p}}\left(\frac{1-\operatorname{Corr}\left(\hat{y}_{p}, y_{p}\right)^{2}}{\operatorname{Corr}\left(\hat{y}_{p}, y_{p}\right) \sigma_{y_{p}}}\right)
\end{array}
$$


Esta es la ecuación (15) de la sección 3.2.

Siguiendo a Jerrim et al. (2014), es posible obtener una expresión más simple para el límite en probabilidad de $\hat{\beta}_{\text {TSTSLS }}$, bajo el supuesto de que la covarianza entre el ingreso predicho del padre y el real es igual a la covarianza entre el ingreso predicho del padre y éste mismo. En este caso, se tiene:

$\operatorname{Corr}\left(y_{p}, \hat{y}_{p}\right)=\frac{\operatorname{Cov}\left(y_{p}, \hat{y}_{p}\right)}{\sigma_{y_{p}} \sigma_{\hat{y}_{p}}}=\frac{\operatorname{Cov}\left(\hat{y}_{p}, \hat{y}_{p}\right)}{\sigma_{y_{p}} \sigma_{\hat{y}_{p}}}=\frac{\sigma_{\hat{y}_{p}}^{2}}{\sigma_{y_{p}} \sigma_{\hat{y}_{p}}}=\frac{\sigma_{\hat{y}_{p}}}{\sigma_{y_{p}}}=R$

donde $\mathrm{R}$ es la raíz cuadrada de la varianza explicada $\left(\mathrm{R}^{2}\right)$ en el modelo de la primera etapa (11). Entonces, el límite en probabilidad del estimador sería:

$$
\begin{aligned}
\operatorname{plim} \hat{\beta}_{\text {TSTSLS }}=\beta+\lambda_{2} \sigma_{\hat{y}_{p}}\left(\frac{1-\left(\sigma_{\hat{y}_{p}} / \sigma_{y_{p}}\right)^{2}}{\left(\sigma_{y_{p}} / \sigma_{y_{p}}\right) \sigma_{y_{p}}}\right) \\
\operatorname{plim} \beta_{\text {TSTSLS }}=\beta+\lambda_{2}\left(1-\frac{\sigma_{\hat{y}_{p}}^{2}}{\sigma_{y_{p}}^{2}}\right) \\
\operatorname{plim} \hat{\beta}_{T S T S L S}=\beta+\lambda_{2}\left(1-R^{2}\right)
\end{aligned}
$$

Esta es la ecuación (16) del texto.

\section{B.5. Límite en probabilidad del estimador OLS con life-cycle bias.}

Dado el modelo definido en las ecuaciones (3), (17) y (18), el límite en probabilidad del estimador OLS de la EII está dado por:

$$
\operatorname{plim} \beta_{O L S}=\frac{\operatorname{Cov}\left(y_{p a}, y_{h a}\right)}{\operatorname{var}\left(y_{p a}\right)}=\frac{\operatorname{Cov}\left(\mu_{a} y_{p}+u_{p a}, \lambda_{a} y_{h i}+u_{h a}\right)}{\operatorname{var}\left(\mu_{a} y_{p}+u_{p a}\right)}
$$

Reemplazando $y_{p}=1 / \mu_{a}\left(y_{p a}-u_{p a}\right)$, entonces:

$$
\begin{aligned}
& \operatorname{plim} \hat{\beta}_{O L S}=\frac{\operatorname{Cov}\left(\mu_{a} y_{p}+u_{p a}, \lambda_{a} y_{h i}+u_{h a}\right)}{\operatorname{var}\left(\mu_{a} y_{p}+u_{p a}\right)} \\
& \operatorname{plim} \beta_{O L S}=\frac{\beta \mu_{a} \lambda_{a}+\mu_{a} \operatorname{Cov}\left(y_{p}, u_{h a}\right)+\lambda_{a} \operatorname{Cov}\left(y_{h}, u_{p a}\right)+\operatorname{Cov}\left(u_{p a}, u_{h a}\right)}{\mu_{\alpha}^{2} \operatorname{var}\left(y_{p}\right)+\operatorname{var}\left(u_{p a}\right)}
\end{aligned}
$$


Suponiendo que $\operatorname{Cov}\left(y_{p}, u_{h a}\right)=\operatorname{Cov}\left(y_{h}, u_{p a}\right)=\operatorname{Cov}\left(u_{p a}, u_{h a}\right)=0$, se tiene:

$$
\operatorname{plim} \beta_{O L S}=\beta \frac{\mu_{a} \lambda_{a}}{\mu_{\alpha}^{2} \operatorname{var}\left(y_{p}\right)+\operatorname{var}\left(u_{p a}\right)}
$$

Ésta es la ecuación (19) de la sección 3.2.

\section{B.6. Límite en probabilidad del estimador IV con life-cycle bias}

Dado el modelo conformado por las ecuaciones (3), (8), (9), (17) y (18), el límite en probabilidad del estimador IV de la EII sería ahora:

$$
\begin{aligned}
& p \lim \beta_{I V}=\frac{\operatorname{Cov}\left(E_{p}, y_{h a}\right)}{\operatorname{Cov}\left(E_{p}, y_{p a}\right)} \\
& p \lim \beta_{I V}=\frac{\operatorname{Cov}\left(E_{p}, \lambda_{a} \delta_{1} y_{p}+\lambda_{a} \delta_{2} E_{p}+\lambda_{a} \varepsilon_{h}+u_{h a}\right)}{\operatorname{Cov}\left(E_{p}, y_{p a}\right)}
\end{aligned}
$$

Si se asume que $\operatorname{Cov}\left(E_{p}, \varepsilon_{h}\right)=0$ y reemplazando $y_{p}=1 / \mu_{a}\left(y_{p a}-u_{p a}\right)$, entonces:

$$
p \lim \hat{\beta}_{I V}=\frac{\operatorname{Cov}\left(E_{p},\left[\frac{\lambda_{a} \delta_{1}}{\mu_{a}}\left(y_{p a}-u_{p a}\right)\right]+\lambda_{a} \delta_{2} E_{p}+u_{h a}\right)}{\operatorname{Cov}\left(E_{p}, y_{p a}\right)}
$$

$p \lim \beta_{I V}=\frac{\operatorname{Cov}\left(E_{p}, \frac{\lambda_{a} \delta_{1}}{\mu_{a}} y_{p a}+\lambda_{a} \delta_{2} E_{p}\right)}{\operatorname{Cov}\left(E_{p}, y_{p a}\right)}+\frac{\operatorname{Cov}\left(E_{p}, u_{h a}\right)-\frac{\lambda_{a} \delta_{1}}{\mu_{a}} \operatorname{Cov}\left(E_{p}, u_{p a}\right)}{\operatorname{Cov}\left(E_{p}, y_{p a}\right)}$

$$
p \lim \hat{\beta}_{I V}=\frac{\lambda_{a}}{\mu_{a}} \delta_{1}+\lambda_{a} \delta_{2} \frac{\operatorname{Var}\left(E_{p}\right)}{\operatorname{Cov}\left(E_{p}, y_{p a}\right)}+\frac{\operatorname{Cov}\left(E_{p}, u_{h a}\right)-\frac{\lambda_{a} \delta_{1}}{\mu_{a}} \operatorname{Cov}\left(E_{p}, u_{p a}\right)}{\operatorname{Cov}\left(E_{p}, y_{p a}\right)}
$$

Reemplazando $\delta_{1}$ a partir de (B.7) que define la relación entre $\beta$ y los parámetros $\delta_{1}$ y $\delta_{2}$ de la ecuación (9), se tiene:

$$
\begin{aligned}
p \lim \hat{\beta}_{I V} & =\frac{\lambda_{a}}{\mu_{a}} \beta+\lambda_{a} \delta_{2}\left(\frac{\operatorname{Var}\left(E_{p}\right)}{\operatorname{Cov}\left(E_{p}, y_{p a}\right)}-\frac{1}{\mu_{a}} \frac{\operatorname{Cov}\left(E_{p}, y_{p a}\right)}{\operatorname{Var}\left(y_{p}\right)}\right) \\
& +\frac{\operatorname{Cov}\left(E_{p}, u_{h a}\right)}{\operatorname{Cov}\left(E_{p}, y_{p a}\right)}-\frac{\lambda_{a} \delta_{1}}{\mu_{a}} \frac{\operatorname{Cov}\left(E_{p}, u_{p a}\right)}{\operatorname{Cov}\left(E_{p}, y_{p a}\right)}
\end{aligned}
$$


Asumiendo que $\operatorname{Cov}\left(E_{p}, u_{p a}\right)=0$, se tiene que

$$
p \lim \beta_{I V}=\frac{\lambda_{a}}{\mu_{a}} \beta+\lambda_{a} \delta_{2} \sigma_{E_{p}}\left[\frac{1-\frac{1}{\mu_{a}} \operatorname{Corr}\left(E_{p}, y_{p a}\right)^{2}}{\operatorname{Corr}\left(E_{p}, y_{p a}\right) \sigma_{y_{p}}}\right]+\frac{\operatorname{Cov}\left(E_{p}, u_{h a}\right)}{\operatorname{Cov}\left(E_{p}, y_{p a}\right)}
$$

Esta es la ecuación (20) de la sección 3.3.

\section{B.7. Límite en probabilidad del estimador TS2SLS con life-cycle bias}

Cuando en el modelo a estimar está conformado por las ecuaciones (3), (11), (14), (17) y (18), el límite en probabilidad del estimador TSTSLS:

$$
p \lim \beta_{T S 2 S L S}=\frac{\operatorname{Cov}\left(\hat{y}_{p}, y_{h a}\right)}{\operatorname{Cov}\left(\hat{y}_{p}, y_{p a}\right)}
$$

$p \lim \beta_{T S 2 S L S}=\frac{\operatorname{Cov}\left(\hat{y}_{p}, \lambda_{a} \lambda_{1} y_{p}+\lambda_{a} \lambda_{2} \hat{y}_{p}+\lambda_{a} \varepsilon_{h}+u_{h a}\right)}{\operatorname{Cov}\left(\hat{y}_{p}, y_{p a}\right)}$

Asumiendo que $\operatorname{Cov}\left(\hat{y}_{p}, \varepsilon_{h}\right)=0$ y reemplazando $y_{p}=1 / \mu_{a}\left(y_{p a}-u_{p a}\right)$, entonces:

$p \lim \beta_{T S 2 S L S}=\frac{\operatorname{Cov}\left(\hat{y}_{p},\left[\frac{\lambda_{a} \lambda_{1}}{\mu_{a}}\left(y_{p a}-u_{p a}\right)\right]+\lambda_{a} \lambda_{2} \hat{y}_{p}+u_{h a}\right)}{\operatorname{Cov}\left(\hat{y}_{p}, y_{p a}\right)}$

$p \lim \hat{\beta}_{T S 2 S L S}=\frac{\operatorname{Cov}\left(\hat{y}_{p}, \frac{\lambda_{a} \lambda_{1}}{\mu_{a}} y_{p a}\right)}{\operatorname{Cov}\left(\hat{y}_{p}, y_{p a}\right)}+\lambda_{a} \lambda_{2} \frac{\operatorname{Cov}\left(\hat{y}_{p}, \hat{y}_{p}\right)}{\operatorname{Cov}\left(\hat{y}_{p}, y_{p a}\right)}+\frac{\operatorname{Cov}\left(\hat{y}_{p}, u_{h a}\right)-\frac{\lambda_{a} \lambda_{1}}{\mu_{a}} \operatorname{Cov}\left(\hat{y}_{p}, u_{p a}\right)}{\operatorname{Cov}\left(\hat{y}_{p}, y_{p a}\right)}$

$p \lim \hat{\beta}_{T S 2 S L S}=\frac{\lambda_{a}}{\mu_{a}} \lambda_{1}+\lambda_{a} \lambda_{2} \frac{\operatorname{Var}\left(\hat{y}_{p}\right)}{\operatorname{Cov}\left(\hat{y}_{p}, y_{p a}\right)}+\frac{\operatorname{Cov}\left(\hat{y}_{p}, u_{h a}\right)-\frac{\lambda_{a} \lambda_{1}}{\mu_{a}} \operatorname{Cov}\left(\hat{y}_{p}, u_{p a}\right)}{\operatorname{Cov}\left(\hat{y}_{p}, y_{p a}\right)}$

Dada la relación entre $\beta$ en (3) y $\lambda_{1}$ y $\lambda_{2}$ en (15) se tiene:

$$
\begin{aligned}
p \lim \hat{\beta}_{T S 2 S L S} & =\frac{\lambda_{a}}{\mu_{a}} \beta+\lambda_{a} \lambda_{2}\left(\frac{\operatorname{Var}\left(\hat{y}_{p}\right)}{\operatorname{Cov}\left(\hat{y}_{p}, y_{p a}\right)}-\frac{1}{\mu_{a}} \frac{\operatorname{Cov}\left(\hat{y}_{p}, y_{p a}\right)}{\operatorname{Var}\left(y_{p}\right)}\right) \\
& +\frac{\operatorname{Cov}\left(\hat{y}_{p}, u_{h a}\right)}{\operatorname{Cov}\left(\hat{y}_{p}, y_{p a}\right)}-\frac{\lambda_{a} \lambda_{1}}{\mu_{a}} \frac{\operatorname{Cov}\left(\hat{y}_{p}, u_{p a}\right)}{\operatorname{Cov}\left(\hat{y}_{p}, y_{p a}\right)}
\end{aligned}
$$


Asumiendo que $\operatorname{Cov}\left(\hat{y}_{p}, u_{p a}\right)=0$, teniendo en cuenta que $\sigma_{\hat{y}_{p}}=\sqrt{\operatorname{Var}\left(\hat{y}_{p}\right)}$ y $\sigma_{y_{p}}=\sqrt{\operatorname{Var}\left(y_{p}\right)}$ entonces (B.13) se reduce a:

$$
p \lim \beta_{T S 2 S L S}=\frac{\lambda_{a}}{\mu_{a}} \beta+\lambda_{a} \lambda_{2} \sigma_{\hat{y}_{p}}\left[\frac{1-\frac{1}{\mu_{a}} \operatorname{Corr}\left(\hat{y}_{p}, y_{p a}\right)^{2}}{\operatorname{Corr}\left(\hat{y}_{p}, y_{p a}\right) \sigma_{y_{p}}}\right]+\frac{\operatorname{Cov}\left(\hat{y}_{p}, u_{h a}\right)}{\operatorname{Cov}\left(\hat{y}_{p}, y_{p a}\right)}
$$

Esta es la ecuación (21) de la sección 3.3.

\section{B.8. Límites en probabilidad de los estimadores del límite inferior y superior de la EII en el modelo intergeneracional alternativo}

El modelo a estimar está definido por las ecuaciones (1), (2), (6) y (22). El parámetro de interés es la EII definida en la ecuación (23) (B*). Según Muller (2010), una forma de obtener un límite superior de esta EII que surge del modelo intergeneracional alternativo es utilizando un promedio del ingreso del padre durante varios años de la niñez o adolescencia de los hijos. Sin embargo, debe recordarse que, dada las limitaciones de información existentes para Argentina, es necesario obtener primero una estimación de ese promedio. La estrategia propuesta en esta investigación consiste en estimar (22) utilizando $\hat{y}_{p}=\hat{\gamma} Z_{p}$ y $\hat{\varepsilon}_{p}$ en lugar del ingreso permanente $\left(y_{p}\right)$ y del ingreso transitorio ( $u_{p}$ ) inobservados del padre. Específicamente, a partir de los pasos descriptos en la sección 3.4 y bajo el supuesto de perfecta correlación entre los componentes transitorios del término de error, es posible obtener $\hat{B}_{L S}$ utilizando una estimación del ingreso paterno en diferentes años $\mathrm{k}$ de la niñez y adolescencia de los hijos, definido por $\widetilde{y}_{p k}=\hat{\gamma}_{p k} X_{p k}+\hat{\varepsilon}_{p k}$.

Asimismo, si se utilizan la educación o la ocupación del padre como variable instrumental $\left(Z_{p}\right)$, dado que probablemente estas variables sean endógenas entonces el ingreso de los hijos estará dado por:

$$
y_{h}=\lambda_{1} y_{p}+\lambda_{2} \frac{\sum_{k} \widetilde{y}_{p k}}{m-r}+\sum_{q} \beta_{q} u_{p q}+u_{h t}
$$


A continuación se deriva el plim del estimador propuesto para el límite superior de la EII que surge del modelo alternativo $\left(\mathrm{B}^{*}\right)$ para cualquier $\delta>0^{132}$ :

$$
p \lim \hat{B}_{L S}=\frac{\operatorname{Cov}\left(\frac{1}{K} \sum_{k} \widetilde{y}_{p k}, y_{h t}\right)}{\operatorname{Cov}\left(\frac{1}{K} \sum_{k} \widetilde{y}_{p k}, \frac{1}{Q} \sum_{q} y_{p q}\right)}
$$

Se analiza en primer lugar el denominador de (B.18), siguiendo las derivaciones de Muller (2008) para el estimador PT del modelo (1), (2), (6) y (22). Teniendo en cuenta que $\widetilde{y}_{p k}=\hat{\gamma}_{p k} X_{p k}+\varepsilon_{p k}=y_{p k}=y_{p}+u_{p k}$ y que $y_{p q}=y_{p}+u_{p q}$, suponiendo que $\mathrm{K}=\mathrm{Q}=\mathrm{T}$, el denominador es igual a:

$$
\begin{aligned}
& D=\operatorname{Cov}\left(\frac{1}{K} \sum_{k}\left(y_{p}+u_{p k}\right), \frac{1}{Q} \sum_{q}\left(y_{p}+u_{p q}\right)\right) \\
& D=\frac{1}{T^{2}} \operatorname{Var}\left(\sum_{k}\left(y_{p}+u_{p k}\right)\right) \\
& \mathrm{D}=\frac{1}{T^{2}} \operatorname{Var}\left(\sum_{k}\left(y_{p}+\left(v_{p k}+\omega_{p k}\right)\right)\right. \\
& \mathrm{D}=\operatorname{Var}\left(y_{p}\right)+(1 / T) \operatorname{Var}\left(v_{p}\right)+(1 / T) \alpha \operatorname{Var}\left(\omega_{p}\right)
\end{aligned}
$$

donde $\alpha=1+2 \delta\left(\frac{T-\delta T-1+\delta^{T}}{T(1-\delta)^{2}}\right)$

No obstante, con relación al supuesto $\mathrm{K}=\mathrm{Q}$ debe aclararse que existen varios escenarios posibles en términos de los solapamientos que pueden presentarse entre el conjunto de los ingresos medidos en la niñez de los hijos (Q) y el conjunto de los ingresos usados en la regresión $(\mathrm{K})$. En el caso en que se utilicen en la regresión una medida del ingreso de los padres para todos los años de la niñez de los hijos, se cumplirá que $\mathrm{K}=\mathrm{Q}$.

Queda por determinar ahora el plim del numerador. Reemplazando $y_{h}=\lambda_{1} y_{p}+\lambda_{2} \frac{\sum_{k} \widetilde{y}_{p k}}{K}+\sum_{q} \beta_{q} u_{p q}+u_{h t}$ y $\tilde{y}_{p k}=y_{p}+u_{p k}$ se tiene:

\footnotetext{
${ }^{132}$ Para no complejizar las ecuaciones se deja de lado en la derivación la consideración del potencial lyfecycle bias. No obstante, es posible mostrar que su efecto en el plim del estimador es similar al analizado previamente bajo el modelo tradicional.
} 


$$
\begin{aligned}
N= & \operatorname{Cov}\left(\frac{1}{K} \sum_{k} \widetilde{y}_{p k}, y_{h t}\right) \\
N= & \frac{1}{K} \operatorname{Cov}\left(\sum_{k} \widetilde{y}_{p k}, \lambda_{1} y_{p}+\lambda_{2} \frac{\sum_{k} \widetilde{y}_{p k}}{K}+\sum_{q} \beta_{q} u_{p q}+u_{h t}\right) \\
N= & \frac{\lambda_{1}}{K} \operatorname{Cov}\left(\sum_{k}\left(y_{p}+u_{p k}\right), y_{p}\right)+\frac{\lambda_{2}}{K^{2}} \operatorname{Var}\left(\sum_{k} \widetilde{y}_{p k}\right) \\
& +\frac{1}{K} \operatorname{Cov}\left(\sum_{k}\left(y_{p}+u_{p k}\right), \sum_{q} \beta_{q} u_{p q}\right)+\frac{1}{K} \operatorname{Cov}\left(\sum_{k}\left(y_{p}+u_{p k}\right), u_{h t}\right)
\end{aligned}
$$

Dado que $\operatorname{Cov}\left(u_{p k}, y_{p}\right)=0 \forall \mathrm{k}, \quad$ suponiendo que $\operatorname{Cov}\left(y_{p}, u_{h t}\right)=0 \forall \mathrm{t} \quad \mathrm{y}$ $\operatorname{Cov}\left(u_{p k}, u_{h t}\right)=0 \forall \mathrm{k}, \mathrm{t}$ y entonces,

$$
\begin{aligned}
N= & \lambda_{1} \operatorname{Var}\left(y_{p}\right)+\frac{\lambda_{2}}{K^{2}} \operatorname{Var}\left(\sum_{k}\left(y_{p}+\omega_{p k}+v_{p k}\right)\right)+\frac{1}{K} \sum_{k} \operatorname{Cov}\left(\sum_{q} \beta_{q}\left(\omega_{p q}+v_{p q}\right)\left(\omega_{p k}+v_{p k}\right)\right) \\
N= & \lambda_{1} \operatorname{Var}\left(y_{p}\right)+\lambda_{2} \operatorname{Var}\left(y_{p}\right)+\frac{\lambda_{2}}{K} \operatorname{Var}\left(v_{p}\right)+\frac{\lambda_{2}}{K} \alpha \operatorname{Var}\left(\omega_{p}\right)+\frac{1}{K} \sum_{k} \operatorname{Cov}\left(\sum_{q} \beta_{q} \omega_{p q}, \omega_{p k}\right) \\
& +\frac{1}{K} \sum_{k} \operatorname{Cov}\left(\sum_{q} \beta_{q} \omega_{p q}, v_{p k}\right)+\frac{1}{K} \sum_{k} \operatorname{Cov}\left(\sum_{q} \beta_{q} v_{p q}, \omega_{p k}\right)+\frac{1}{K} \sum_{k} \operatorname{Cov}\left(\sum_{q} \beta_{q} v_{p q}, v_{p k}\right) \\
N= & \lambda_{1} \operatorname{Var}\left(y_{p}\right)+\lambda_{2} \operatorname{Var}\left(y_{p}\right)+\frac{\lambda_{2}}{K} \operatorname{Var}\left(v_{p}\right)+\frac{\lambda_{2}}{K} \alpha \operatorname{Var}\left(\omega_{p}\right) \\
& +\frac{1}{K} \sum_{k} \operatorname{Cov}\left(\sum_{q} \beta_{q} \omega_{p q}, \omega_{p k}\right)+\frac{1}{K} \sum_{k} \operatorname{Cov}\left(\sum_{q} \beta_{q} v_{p q}, v_{p k}\right) \\
N= & \lambda_{1} \operatorname{Var}\left(y_{p}\right)+\lambda_{2} \operatorname{Var}\left(y_{p}\right)+\frac{\lambda_{2}}{K} \operatorname{Var}\left(v_{p}\right)+\frac{\lambda_{2}}{K} \alpha \operatorname{Var}\left(\omega_{p}\right) \\
& +\frac{1}{K} \sum_{k} \sum_{q} \beta_{q} \operatorname{Cov}\left(\omega_{p q}, \omega_{p k}\right)+\frac{1}{K} \sum_{k} \sum_{k} \beta_{k} \operatorname{Cov}\left(v_{p k}, v_{p k}\right)
\end{aligned}
$$

Teniendo en cuenta que $v_{p q} \mathrm{y} v_{p k}$ son componentes de ruido blanco, entonces $\operatorname{Cov}\left(v_{p q}, v_{p k}\right)=0 \forall q \neq k$

$$
\begin{aligned}
N= & \lambda_{1} \operatorname{Var}\left(y_{p}\right)+\lambda_{2} \operatorname{Var}\left(y_{p}\right)+\frac{\lambda_{2}}{K} \operatorname{Var}\left(v_{p}\right)+\frac{\lambda_{2}}{K} \alpha \operatorname{Var}\left(\omega_{p}\right) \\
& +\frac{1}{K} \sum_{k} \sum_{q} \beta_{q} \operatorname{Cov}\left(\omega_{p q}, \omega_{p k}\right)+\frac{1}{K} \sum_{k} \beta_{k} \operatorname{Var}\left(v_{p k}\right)
\end{aligned}
$$

Dado que $\omega_{p q}$ sigue un proceso $\operatorname{AR}(1)$, de acuerdo con las derivaciones de Muller (2008) se tiene: 
$\operatorname{Cov}\left(\omega_{p q}, \omega_{p k}\right)=\delta^{|q-k|} \operatorname{var}\left(\omega_{p q}\right)$

Por tanto,

$$
\begin{aligned}
\sum_{k} \sum_{q} \beta_{q} \operatorname{Cov}\left(\omega_{p q}, \omega_{p k}\right) & =\operatorname{var}\left(\omega_{p q}\right) \sum_{k} \sum_{q} \beta_{q} \delta^{|q-k|} \\
& =\operatorname{var}\left(\omega_{p q}\right) \sum_{q} \beta_{q} \sum_{k} \delta^{|q-k|}
\end{aligned}
$$

Supongamos que $\mathrm{K}=\mathrm{m}-\mathrm{r}$, donde $\mathrm{m}$ es el último año $\mathrm{y} r$ es el primer año de la niñez de los hijos, entonces:

$$
\operatorname{var}\left(\omega_{p q}\right) \sum_{q} \beta_{q} \sum_{k} \delta^{|q-k|}=\operatorname{var}\left(\omega_{p q}\right) \sum_{q} \beta_{q}\left(\delta^{|q-r|}+\delta^{|q-r-1|}+\delta^{|q-r-2|}+\ldots+\delta^{|q-m+1|}+\delta^{|q-m|}\right)
$$

En el caso en que $\mathrm{K}=\mathrm{Q}$, la serie entre paréntesis es primero creciente y luego decreciente en $\delta^{|q-k|}$.

Para un año q dado dividimos la suma entre paréntesis en dos series geométricas finitas como seguidos.

$$
\begin{aligned}
& \Lambda_{q}=\left(\delta^{|q-k|} \frac{1-\left(\frac{1}{\delta}\right)^{|q-r|}}{1-\frac{1}{\delta}}\right)+\left(\delta^{0} \frac{1-\delta^{|q-m|+1}}{1-\delta}\right) \\
& \Lambda_{q}=\delta^{|q-r|} \times \frac{\frac{\delta^{|q-r|}-1}{\delta^{|q-r|}}}{\frac{\delta-1}{\delta}}+\frac{1-\delta^{|q-m|+1}}{1-\delta} \\
& \Lambda_{q}=\frac{\delta\left(\delta^{|q-r|}-1\right)}{\delta-1}+\frac{\delta^{|q-m|+1}-1}{\delta-1} \\
& \Lambda_{q}=\frac{1+\delta-\delta^{|q-r|+1}-\delta^{|q-m|+1}}{1-\delta} \\
& \sum_{k} \sum_{q} \beta_{q} \operatorname{Cov}\left(\omega_{p q}, \omega_{p k}\right)=\operatorname{var}\left(\omega_{p q}\right) \sum_{q} \beta_{q} \sum_{k} \delta^{|q-k|} \\
& =\operatorname{var}\left(\omega_{p q}\right) \sum_{q} \beta_{q} \Lambda_{q}
\end{aligned}
$$

Por otra parte, la relación entre los parámetros $\beta$ у $\lambda_{1}$ y $\lambda_{2}$ es la siguiente: 


$$
\begin{aligned}
& \beta=\frac{\operatorname{Cov}\left(y_{h}, y_{p}\right)}{\operatorname{Var}\left(y_{p}\right)} \\
& \beta=\lambda_{1}+\lambda_{2} \frac{\operatorname{Cov}\left(\frac{\sum_{k} \widetilde{y}_{p k}}{K}, y_{p}\right)}{\operatorname{Var}\left(y_{p}\right)}+\frac{\operatorname{Cov}\left(\sum_{q} \beta_{q} u_{p q}, y_{p}\right)}{\operatorname{Var}\left(y_{p}\right)}+\frac{\operatorname{Cov}\left(u_{h t}, y_{p}\right)}{\operatorname{Var}\left(y_{p}\right)}
\end{aligned}
$$

Teniendo en cuenta los siguientes supuestos: $\operatorname{Cov}\left(u_{p q}, y_{p}\right)=0 \forall q$ y $\operatorname{Cov}\left(u_{p t}, y_{p}\right)=0 \forall t$ , se tiene

$$
\begin{aligned}
& \beta=\lambda_{1}+\frac{\lambda_{2}}{K} \frac{\operatorname{Cov}\left(\sum_{k}\left(y_{p}+u_{p k}\right), y_{p}\right)}{\operatorname{Var}\left(y_{p}\right)} \\
& \beta=\lambda_{1}+\frac{\lambda_{2}}{K} \frac{\operatorname{KVar}\left(y_{p}\right)}{\operatorname{Var}\left(y_{p}\right)} \\
& \beta=\lambda_{1}+\lambda_{2} \text { (B.22) }
\end{aligned}
$$

Reemplazando (B.21) y (B.22) en (B.20) se tiene:

$$
N=\beta \operatorname{Var}\left(y_{p}\right)+\frac{\lambda_{2}}{K} \operatorname{Var}\left(v_{p}\right)+\frac{\lambda_{2}}{K} \alpha \operatorname{Var}\left(\omega_{p}\right)+\frac{1}{K} \operatorname{Var}\left(\omega_{p q}\right) \sum_{q} \beta_{q} \Lambda_{q}+\frac{1}{K} \sum_{k} \beta_{k} \operatorname{Var}\left(v_{p k}\right)
$$

Por lo tanto, de (B.19) y (B.23) se obtiene finalmente:

$$
p \lim \hat{B}_{L S}^{*}=\frac{\beta \operatorname{Var}\left(y_{p}\right)+\lambda_{2} / T \operatorname{Var}\left(v_{p}\right)+\lambda_{2} / T \alpha \operatorname{Var}\left(\omega_{p}\right)+1 / T \operatorname{var}\left(\omega_{p q}\right) \sum_{q} \beta_{q} \Lambda_{q}+1 / T \sum_{k} \beta_{k} \operatorname{Var}\left(v_{p k}\right)}{\operatorname{Var}\left(y_{p}\right)+(1 / T) \operatorname{Var}\left(v_{p}\right)+(1 / T) \alpha \operatorname{Var}\left(\omega_{p}\right)}
$$

donde $\Lambda_{q}=\frac{1+\delta-\delta^{|q-r|+1}-\delta^{|q-m|+1}}{1-\delta}$ y $\alpha=1+2 \delta\left(\frac{T-\delta T-1+\delta^{T}}{T(1-\delta)^{2}}\right)$

Por otra parte, como se mencionó previamente en el texto, teniendo en cuenta que la EII propuesta por este nuevo modelo es la suma entre $\beta$ y las elasticidades correspondientes al componente transitorio del ingreso paterno, el estimador TSIV ofrece un límite inferior de B* pues sólo captura la elasticidad referida al componente permanente. Dado el modelo definido en (1), (2), (6) y (22) y el límite en probabilidad del estimador TS2SLS dado en (B.11) se tiene que:

$$
p \lim \hat{B}_{L I}^{*}=\beta+\lambda_{2} \sigma_{\hat{y}_{p}}\left[\frac{1-\operatorname{Corr}\left(\hat{y}_{p}, y_{p a}\right)^{2}}{\operatorname{Corr}\left(\hat{y}_{p}, y_{p a}\right) \sigma_{y_{p}}}\right]
$$


Bajo el supuesto de que $\lambda_{2}=0$, se observa a partir de (B.25) que este estimador ofrece una medida de la elasticidad intergeneracional del ingreso permanente y, entonces, constituye un límite inferior del parámetro de interés, B* dado por (23). En tanto que, según (B.24) $\hat{B}_{L S}$ constituye un límite superior de B*. Asimismo, debe observarse que esto también ocurre cuando $\lambda_{2}>0$. Ahora bien, como ya se mencionó, cuando se utiliza como instrumento para predecir el ingreso del padre su educación, es razonable suponer que su efecto sobre el ingreso del hijo capturado por $\lambda_{2}$ es positivo. Además, teniendo en cuenta que según los resultados obtenidos por Sewell y Hauser (1975), Corcoran et al. (1992), Mazumder (2005), el efecto directo de la educación del padre en el ingreso del hijo no es estadísticamente distinto de cero, una vez que se controla por el ingreso del padre promediado durante varios años, el supuesto de que $\lambda_{2}=0$ también resulta razonable. 


\section{Capítulo $3^{*}$}

\section{Movilidad intergeneracional y gasto público. Evidencia para} Argentina

\section{Introducción}

En los últimos años, la mayoría de la literatura sobre MI, particularmente, la que analiza la movilidad intergeneracional del ingreso (MII) se ha concentrado en obtener una medida adecuada del nivel de MI, siendo relativamente más escasas las investigaciones que avanzaron en la identificación de los factores causales subyacentes al proceso de transmisión del ingreso entre generaciones. Sin embargo, comprender los mecanismos subyacentes a las correlaciones intergeneracionales es crucial para el desarrollo de políticas públicas apropiadas que promuevan el objetivo social relevante en materia de equidad. Por esto, un estudio de la transmisión intergeneracional del ingreso y de sus determinantes puede aportar información útil para el diseño de políticas adecuadas.

Según los modelos teóricos que analizan los factores asociados con la MII (Mulligan, 1997; Solon, 2004; Ichino et al., 2011) ${ }^{133}$, no sólo la inversión de los padres en sus hijos es uno de los principales canales de transmisión intergeneracional de ingresos, sino también la inversión del gobierno en el capital humano de los niños y adolescentes. Por esto, el principal objetivo de esta investigación es determinar el efecto que tiene en la movilidad intergeneracional de ingresos en Argentina, el gasto público, concretamente, el realizado por los tres niveles de gobiernos (nacional, provincial y municipal) que pueden modificar las inversiones públicas en el capital humano de los

\footnotetext{
- Agradezco a Leonardo Gasparini por sus valiosos comentarios y observaciones. También agradezco las sugerencias realizadas por el Comité de Doctorado, por los asistentes a los Seminarios de Doctorado de la UNLP y por Ana Inés Navarro en la XLVII Reunión Anual de la AAEP que han enriquecido versiones preliminares de este capítulo.

${ }^{133}$ Ver sección 2 para más detalles.
} 
niños y adolescentes. Específicamente, se busca evaluar si el gasto público cumple con uno de los objetivos centrales de la política social que es incrementar la movilidad económica intergeneracional y la igualdad de oportunidades, disminuyendo el impacto del origen socioeconómico en el ingreso obtenido en la adultez. Asimismo, se pretende examinar si la brecha de movilidad observada entre los hijos que provienen de distintos trasfondos socioeconómicos familiares es menor para aquellos que experimentaron mayores niveles de gasto público durante su adolescencia.

Los estudios empíricos que examinan la relación entre movilidad intergeneracional y gasto público son escasos a nivel internacional $\mathrm{y}$, prácticamente inexistentes, a nivel nacional. Entre los antecedentes más directos de esta investigación se encuentran los estudios de Mayer y Lopoo (ML) (2008) para Estados Unidos y Behrman, Gaviria y Székeley (BGS) (2001) para 19 países latinoamericanos y Estados Unidos. A diferencia de estos estudios que se concentran en una medida de posición socioeconómica, el ingreso, en el caso del primero y la educación, en el caso del segundo, en esta investigación se examina el efecto del gasto público tanto en la movilidad intergeneracional del ingreso como en la MI educativa (MIE). La variable educación presenta varias ventajas sobre los ingresos en términos de estimación, especialmente en países como Argentina con falta de información de largo plazo para generaciones sucesivas de una misma familia. Esto genera mayores problemas de estimación cuando la variable de resultado utilizada es el ingreso porque el análisis de la MII requiere observar el ingreso de padres e hijos en momentos similares de sus ciclos vitales, preferentemente en sus edades centrales. Si bien existen métodos, como la técnica Two-Sample Instrumental Variable (TSIV) implementada en esta investigación, que pueden aliviar estos inconvenientes ${ }^{134}$, los supuestos en los que se basa la consistencia de las estimaciones así obtenidas podrían resultar demasiado restrictivos. Además, el examen de la MIE es de interés en sí mismo puesto que los logros educativos ofrecen una fuente importante de información sobre el status y bienestar socioeconómico de los individuos. De esta forma, el análisis de la correlación entre la educación de padres e hijos constituye una alternativa informativa y factible al análisis basado en el ingreso para examinar la transmisión socioeconómica intergeneracional (Hertz et al., 2009). Además, a diferencia de los estudios mencionados que sólo

\footnotetext{
${ }^{134}$ En la sección 3 se describe esta técnica de estimación.
} 
analizan el efecto del gasto total (en el caso de ML) o del gasto en educación (en el caso de BGS), en este investigación se consideran distintas categorías y agregados del gasto público consolidado (GPC), particularmente del gasto público social, con objetivo de explorar el efecto que diferentes medidas de la inversión pública en el capital humano de niños y adolescentes tienen en la movilidad intergeneracional.

Por otro parte, la estrategia de identificación empleada en esta investigación se basa fundamentalmente en explotar la variabilidad temporal del gasto público, de forma similar a como lo hacen ML y BGS. Sin embargo, a diferencia de estos estudios, en los modelos estimados se incluyen, además de efectos fijos locales, un amplio conjunto de variables de control a nivel local que capturan características variables en el tiempo (o entre cohortes) potencialmente relacionadas con el gasto público y con las variables de resultados (ingresos y educación) como, por ejemplo, la recaudación tributaria provincial y la carga tributaria nacional. Así, si no se incluyeran estos controles por provincia, cualquier diferencia en la movilidad intergeneracional por niveles de gasto podría ser parcialmente endógena, entre otras razones, porque, dada la misma tasa de impuestos, los cambios en los ingresos de los residentes en una provincia podrían traducirse en cambios en la recaudación y en el gasto público. También se incluyen controles por otras características provinciales que varían, no sólo localmente, sino también en el tiempo como, por ejemplo, la tasa de pobreza por NBI, el nivel de desigualdad o la tasa de mortalidad infantil existente en la provincia de residencia de cada hijo adulto cuando era adolescente o joven. Estos controles resultan relevantes porque algunas categorías de gasto público, particularmente del gasto público social, se ajustan a los cambios en las necesidades existentes de manera que las provincias con más familias pobres tenderán a gastar más que las provincias con menos familias pobres. La omisión de estas variables de control podrían sesgar las estimaciones del término de interacción entre la posición socioeconómica de los padres y el gasto público que captura el efecto que éste último tiene en las medidas de persistencia intergeneracional consideradas.

Los resultados obtenidos sugieren que el gasto público tiene el efecto esperado, incrementando la movilidad intergeneracional tanto del ingreso como la educativa. Sin embargo, no todas las categorías de gasto son igualmente efectivas para conseguir este resultado. Así, se observa una disminución mayor en la persistencia socioeconómica 
entre generaciones cuando se incrementan las categorías del GPC más directamente relacionadas con el capital humano de los niños y adolescentes: el gasto en salud, nutrición y educación.

Este artículo está organizado de la siguiente manera. En la sección 2 se describe brevemente el marco teórico así como los principales antecedentes de esta investigación, destacando su contribución a la literatura existente. A continuación, en la sección 3 se analizan el modelo empírico, el método de estimación y la estrategia de identificación utilizada para examinar la relación entre movilidad intergeneracional y gasto público. La sección 4 contiene una descripción de las fuentes de datos utilizadas. En la sección 5 se presentan y analizan los resultados obtenidos, para terminar, luego, en la última sección, con las principales conclusiones de la investigación.

\section{Modelos teóricos y antecedentes empíricos}

El marco teórico de este capítulo está conformado por los modelos de persistencia entre generaciones que examinan el efecto del gasto público o, en general, de las acciones estatales en el proceso de transmisión intergeneracional. Entre ellos se encuentra el de Mulligan (1997) que desarrolla un modelo de las preferencias de los padres en el que analiza la transmisión del status económico de una generación a otra. En este modelo, los padres determinan el grado de su preocupación altruista por sus hijos y, conforme a ello, les dedican tiempo y recursos. En este contexto, la autora, describe el efecto de un programa social del gobierno sobre el altruismo de los padres y el bienestar de los niños. Mulligan (1997) muestra que aunque los hijos de familias beneficiarias de subsidios estatales pueden beneficiarse de los recursos adicionales ofrecidos por el programa social, éste puede afectar la formación del altruismo intergeneracional de los padres. En la medida en que las acciones públicas a favor de los niños se financian con impuestos, su efecto neto depende de la forma en que se introducen esos impuestos. Así, si éstos son cargados, en parte, a las familias que reciben el subsidio, puede producirse un efecto negativo sobre el altruismo de los padres y el consumo de sus hijos. En cambio, si los impuestos son cargados a las familias ricas

y el programa público es dirigido hacia las familias pobres, la introducción del 
programa incrementaría el consumo y el altruismo de los pobres, al tiempo que disminuiría el consumo y altruismo de los ricos.

Un modelo teórico que se destaca en el contexto de esta investigación es el desarrollo por Solon (2004) que extiende el modelo seminal de Becker y Tomes $(1979)^{135}$ y describe, en un esquema de optimización simple, el fundamento teórico del enfoque empírico estándar consistente en medir la movilidad del ingreso entre generaciones utilizando la elasticidad intergeneracional del ingreso (EII, en adelante). Una contribución importante de este modelo es la incorporación del efecto de la inversión pública en el capital humano de los niños. Específicamente, el modelo muestra que cuanto más progresiva sea esta inversión del gobierno - en el sentido de que la ratio entre inversión pública e ingreso de los padres disminuya con el ingreso parental - menor será la EII y, por tanto, mayor será la movilidad intergeneracional del ingreso, ceteris paribus.

A partir del modelo seminal de Becker y Tomes (1979) puede derivarse también un marco teórico para comprender los determinantes de los logros educativos de los hijos y cómo las acciones de política pública pueden afectar las correlaciones educativas intergeneracionales. La política pública en gran medida define las elecciones educativas disponibles para padres e hijos, afectando el acceso legal y práctico a la escolaridad así como la calidad y costos de la educación. De esta manera, las acciones del gobierno pueden modificar la oferta y la demanda de educación. Así, por ejemplo, el incremento del gasto público en educación o en capital humano, en general y las mejoras en la calidad de las escuelas públicas son medidas que tienen un impacto en el lado de la oferta. En tanto que las acciones gubernamentales que reducen las restricciones financieras que enfrentan los hogares para invertir en capital humano impactan en la demanda educativa (Behrman y Gaviria, 2001). Las decisiones políticas pueden afectar también el retorno económico de la educación y de otras formas de inversiones del hogar en el capital humano de los hijos. Los padres entonces toman decisiones respecto de las inversiones de tiempo y dinero que realizan en la educación de sus hijos

135 Becker and Tomes (1979) desarrollaron el modelo económico original sobre movilidad intergeneracional del ingreso, basado en la idea de que cada familia maximiza una función de utilidad que incorpora el bienestar de varias generaciones. En este esquema el ingreso de los hijos está determinado por las inversiones que realizan sus padres en ellos y por las denominadas "dotaciones" que reciben los hijos, determinadas genéticamente, como la raza, habilidad y otras características, así como aquellas conformadas por las conexiones familiares y los valores o conocimientos dados por el entorno familiar. 
considerando sus restricciones presupuestarias ${ }^{136}$. Los hijos, por su parte, toman decisiones sobre el esfuerzo educativo que realizarán en función, entre otros factores, de los costos y beneficios percibidos que, a su vez, dependen en parte de las percepciones de las condiciones existentes en el mercado laboral (Hertz et al., 2009).

Otro modelo teórico más reciente que incorpora endógenamente las inversiones públicas en capital humano es el que proponen Ichino et al. (2011). Este esquema teórico muestra que la EII en cualquier sociedad puede estar influenciada por las preocupaciones e intereses en la política económica. Así, las sociedades donde las familias mejor posicionadas económicamente tienen una mayor influencia en el proceso político, tenderán a tener menores inversiones redistributivas en el capital humano y por tanto, mayores niveles de persistencia intergeneracional ${ }^{137}$.

Como se afirmó previamente, los estudios empíricos que examinan la relación entre movilidad intergeneracional y gasto público son escasos a nivel internacional y casi inexistentes, a nivel nacional. Los dos antecedentes más directo de esta investigación son los estudios de Mayer y Lopoo (2008) y Behrman et al. (2001). En el primero, ML utilizando los datos del Panel Study of Income Dynamics (PSID) encuentran que la EII es mayor en los estados de Estados Unidos con un menor gasto por niño que en aquellos que presentan un mayor nivel de gasto público. La estrategia de identificación se basa, principalmente, en el cambio temporal observado en los niveles de gasto público por niño ${ }^{138}$. Como el modelo empírico incluye efectos fijos por el estado en el que residían los hijos cuando tenían 15 años, las estimaciones obtenidas no surgen de la variación cross-section en los niveles de gasto entre los estados. El estudio de BGS, por su parte, examina los efectos del gasto público en educación como porcentaje del PBI sobre el índice de correlación educativa propuesto por Dahan y

\footnotetext{
${ }^{136}$ El acceso al mercado crediticio puede afectar también esta decisión, principalmente en los niveles de educación más elevados.

${ }^{137}$ Según Ichino et al. (2011) y contrariamente a lo que se asume en general, una baja correlación entre el ingreso paterno y el de los hijos no es siempre deseable pues puede implicar más ineficiencia debido a los efectos distorsionadores de las políticas públicas dirigidas a incrementar la movilidad. Además, tomando en cuenta la heterogeneidad en las preferencias por la movilidad intergeneracional, llegan a la conclusión de que incluso si una sociedad completamente móvil es deseable ex ante, puede no ser políticamente sustentable ex post.

${ }^{138}$ Los autores computan el gasto público por niño en cada año dividiendo los gastos totales por la población de 0 a 17 años existente en cada estado, en cada año. Luego, promedian este gasto estatal por niño durante los 3 años en los que los hijos adultos tenían entre 15 a 17 años.
} 
Gaviria $(2001)^{139}$ para 19 países latinoamericanos y Estados Unidos. Los autores estiman un modelo de regresión que tiene como variable dependiente el índice de movilidad educativa intergeneracional y como variables explicativas el gasto público en educación, el PBI per cápita y los años de escolaridad de la población en edad de trabajar. La estrategia de identificación está basada en un modelo de panel con efectos fijos por país. Los resultados obtenidos muestran que un mayor gasto en educación está asociado con valores substancialmente menores del índice de movilidad lo que sugiere que dedicar más recursos a la educación pública incrementa la movilidad intergeneracional. No obstante, si bien la estrategia de estimación por efectos fijos elimina el sesgo potencial de factores inobservables invariantes en el tiempo, las estimaciones podrían aún estar sesgadas por la presencia de características omitidas de los países que varían en el tiempo y que podrían estar correlacionadas con el gasto público así como con el índice de movilidad educativa.

Por su parte Ichino et al. (2011), computan la correlación entre la EII estimada en 10 países (Dinamarca, Finlandia, Canadá, Suecia, Alemania, Francia, Estados Unidos, Reino Unido, España y Australia) con el gasto público en educación que resulta igual a -0.54. La correlación es incluso más fuerte cuando consideran el gasto público en educación primaria. Además, encuentran una elevada correlación cross-country positiva entre la brecha pobres-ricos en la participación política (medida por la pertenencia a un partido político) y la elasticidad intergeneracional del ingreso. Estos resultados sugieren que las preocupaciones e intereses de los individuos por la política económica son importantes en la determinación de una política y, por tanto, influyen en la EII. Sin embargo, la evidencia empírica obtenida por Ichino et al. (2011) está basada en información cross-section de solo 12 países, por lo que es solamente sugestiva.

Otros estudios relacionados con esta investigación, aunque no tan directamente como los anteriores, son los que analizan el efecto de cambios en políticas públicas sobre los niveles de movilidad intergeneracional. Entre ellos se encuentran los de Mulligan (1999), Machin (2007) y Pekkarinen et al. (2009) que examinan la relación entre las políticas educativas y la movilidad intergeneracional ${ }^{140}$. En el primero,

\footnotetext{
${ }^{139}$ Este índice está basado en la correlación de la escolaridad entre hermanos y mide el grado con el cual sus resultados educativos pueden ser explicados por el trasfondo familiar

${ }^{140}$ Davies et al. (2005) desarrollan un modelo teórico en el que examinan el impacto de variaciones de los sistemas educativos en la movilidad intergeneracional.
} 
Mulligan (1999) estima grados similares de movilidad intergeneracional para estados individuales en los Estados Unidos que tienen políticas diferentes para la provisión de educación pública. Machin (2007), por su parte, muestra que la expansión de la educación superior en el Reino Unido en las décadas de los 80s y 90s llevó a un gran incremento en la proporción de hijos de familias de altos ingresos con un grado universitario, pero produjo un aumento mucho menor entre los hijos de familias de bajos ingresos. Además, el autor reporta una movilidad del ingreso mayor para las cohortes nacidas en 1958 que para aquellas nacidas en las décadas subsecuentes, sugiriendo un vínculo entre el acceso a la educación superior y la movilidad intergeneracional. En un estudio empírico reciente, Pekkarinen et al. (2009) examinan los efectos sobre la EII de cambiar el sistema educativo finlandés de uno selectivo a uno más comprehensivo ${ }^{141}$. La estrategia de identificación implementada consiste en explotar la forma en que se implementó la reforma, durante la década de 1970 en diferentes regiones y distintos momentos durante un período de 6 años. En el modelo estimado, la EII depende del sistema educativo experimentado por el niño, controlando por efectos de la cohorte de nacimiento y regionales. Los autores encuentran que la EII para hijos varones cayó de 0.3 a 0.23 como resultado de la reforma educativa, sugiriendo que el anterior ciclo escolar reduce la movilidad intergeneracional. Sin embargo, como la reforma también implicó cambios en los planes de estudios e incrementó la asistencia a los establecimientos educativos, la interpretación exacta de las razones de su impacto en la movilidad intergeneracional no es tan directa. No obstante, el estudio ofrece evidencia empírica robusta de que el sistema educativo importa para la movilidad intergeneracional, medida a través de la EII (Black y Deveraux, 2011).

\footnotetext{
${ }^{141}$ En Finlandia, antes de la reforma educativa implementada en la década de los 70, todos los estudiantes ingresaban a la escuela primaria a los 7 años. Después de 4 años en la escuela primaria, los estudiantes podían aplicar a una escuela secundaria general o continuar en la escuela primaria por dos años más y, en algunas escuelas, continuar con clases durante dos años más. Pero, los estudiantes que seguían esta última trayectoria educativa eran los que no aplicaron o no fueron admitidos en las escuelas secundarias generales ni en los estudios de nivel universitarios, entonces seguían con algún entrenamiento vocacional o abandonaban los estudios. La reforma reemplazó este sistema con una escuela comprehensiva de nueve años a la que todos los niños asistían hasta los 16 años. Luego, los estudiantes elegían si aplicar a las denominadas escuelas secundarias superiores o a las escuelas vocacionales y la admisión a estas dos opciones educativas estaba basada solamente en los grados aprobados en el nivel anterior. Entonces, el ciclo académico y vocacional comienza mucho más tarde después de la reforma (Pekkarinen et al., 2009).
} 
Los estudios que examinan los efectos de las políticas públicas sobre la movilidad educativa intergeneracional son, como señala Hertz et al. (2009), escasos pero crecientes. Entre ellos se encuentra el de Lillard y Willis (1994) que muestra cómo las intervenciones gubernamentales en Malasia redujeron significativamente el efecto de la educación de los padres durante el período 1950-80. Los estudios de Duflo (2001) y Hertz y Jayasundera (2007) analizan los efectos de un programa masivo de construcción de escuelas, encontrando efectos positivos tanto en los logros educativos de los niños beneficiados como en la movilidad educativa. Por su parte, Deng y Treiman (1997) mostraron que las políticas anti-elite de la revolución cultural china redujeron la transmisión educativa intergeneracional en el corto plazo. Finalmente, Hertz et al. (2009) analizan el efecto de las grandes reformas económicas y políticas introducidas en Bulgaria desde 1989 sobre la correlación educativa entre padres e hijos, observando que esta medida de persistencia intergeneracional se duplicó entre 1995 y 2001. Por tanto, los autores concluyen que la crisis económica y fiscal de 1990 llevó a una marcada disminución en la movilidad educativa.

Con relación a los estudios de movilidad intergeneracional en Argentina, como se mencionó en los capítulos anteriores, la mayoría no examina la movilidad del ingreso sino la movilidad ocupacional o educativa entre generaciones ${ }^{142}$.

\section{Metodología}

El modelo empírico tradicional considerado en la literatura para medir el grado de persistencia socioeconómica promedio entre generaciones es el siguiente:

$$
y_{h i}=\beta y_{p i}+\varepsilon_{h i}(1)
$$

donde $y_{h i}$ y $y_{p i}$ son medidas de la posición socioeconómica de hijos y padres, respectivamente. Cuando se considera como medida de status económico el ingreso, entonces el modelo intergeneracional (1) relaciona el logaritmo del ingreso permanente de hijos y padres, siendo $\beta$ la elasticidad intergeneracional del ingreso (EII), esto es,

\footnotetext{
${ }^{142}$ En el Capítulo 1 y en el Capítulo 2 puede consultarse la revisión de los estudios sobre movilidad intergeneracional de la educación y del ingreso en Argentina, respectivamente.
} 
una medida del grado de persistencia del ingreso permanente de padres e hijos. En tanto que, cuando la educación se utiliza como medida de posición socioeconómica, el modelo (1) relaciona los años de educación de los hijos con el de los padres, siendo $\beta$ en este caso un coeficiente que mide la persistencia educativa intergeneracional (CPE en adelante). Este modelo asume implícitamente que la variable utilizada para medir la posición socioeconómica del padre capturará el efecto directo de ésta en la posición del hijo así como el impacto de factores omitidos correlacionados con ambas como características y atributos genéticos de los padres (o la educación del padre en el caso del modelo de MII). Por lo tanto, como ya se mencionó en los capítulos previos, ni la EII ni el CPE constituyen (ni se espera que sean) una medida del efecto causal de la posición socioeconómica de los padres en el resultado económico de los hijos considerado en cada caso. En general, es inevitable que ambos indicadores sobrestimen cualquier relación causal que exista como resultado de la correlación entre el ingreso o la educación de los padres y otras variables explicativas en la ecuación estructural del resultado económico de los hijos. No obstante, aun así, la EII y la CPE constituyen medidas relevantes y ampliamente utilizadas de inmovilidad intergeneracional.

Dado que el objetivo de esta investigación es examinar cómo el gasto público afecta la EII, el modelo a estimar es el siguiente:

$$
\begin{aligned}
Y_{h i, c j t}= & \alpha+\beta Y_{p i, c j}+\delta_{1} G_{c j}+\delta_{2}\left(Y_{p i, c j} \times G_{c j}\right)+\gamma A_{i c j}+\theta X_{c j} \\
& +\varphi D_{j}+\pi D_{t}+\vartheta\left(D_{t} \times D_{j}\right)+\lambda D_{c}+\varepsilon_{i c j t}
\end{aligned}
$$

donde $\mathrm{c}$ indexa la cohorte de nacimiento, $\mathrm{j}$ la provincia de residencia, y $\mathrm{t}$ el año, $Y_{h i, c j t} \mathrm{y}$ $Y_{p i, c j}$ son las variables que mide la posición socioeconómica del hijo y padre (el logaritmo del ingreso familiar, en el modelo de MII o los años de educación, en el modelo MIE); $G_{c j}$ es el logaritmo del gasto público per cápita experimentado por la cohorte c en una etapa determinada de su ciclo de vida (se obtiene como el gasto público per cápita promedio durante los años $\mathrm{t}$ en que los individuos de cada cohorte eran adolescentes o jóvenes); $A_{i c j}$ son controles individuales que incluyen el sexo del hijo, un polinomio de grado cuarto en su edad y en la edad del padre; $X_{c j}$ es un vector de variables de control a nivel provincial que varían por cohorte y en la especificación más completa del modelo incluye los recursos tributarios provinciales, la carga tributaria nacional por provincia, la tasa de desocupación, el nivel de desigualdad 
medido por el coeficiente de Gini, la tasa de crecimiento del Producto Bruto Geográfico (PBG), la tasa de pobreza por NBI, la tasa de asistencia a cada nivel educativo, los años de educación promedio de la población en edad de trabajar (PET), la cantidad total de población, la proporción de la población sin cobertura de salud, la tasa de mortalidad infantil (por mil nacidos vivos), la tasa de mortalidad total (por mil habitantes), el porcentaje de la población sin acceso a agua corriente y sin acceso a desagües cloacales, el partido político del gobernador de cada provincia en relación con el del presidente de turno $^{143} \mathrm{y}$, cuando se considera el gasto público en alguna categoría particular, se incluye como variable de control la proporción del gasto destinada al resto de las categorías no consideradas; $D_{j}$ es un conjunto de dummies por provincia de residencia (efectos fijos por lugar); $D_{t}$ son dummies por año (efectos fijos por año), $D_{c}$ son dummies por cohorte de nacimiento (efectos fijos por cohorte) y $D_{t} \times D_{j}$ es el término de interacción entre las dummies locales y temporales que se introduce para capturar posibles tendencias específicas por provincia en el ingreso de los hijos que podrían estar correlacionadas con los cambios ocurridos en el gasto público. El coeficiente de interés, en este caso, es $\delta_{2}$ que corresponde al término de interacción entre el ingreso parental y el gasto público y captura el efecto de este último sobre la EII. Es importante destacar que la incorporación de efectos fijos por provincia implica que la estrategia de identificación subyacente al modelo (2) se basa, principalmente, en el cambio temporal (por cohorte de nacimiento) observado en los niveles de gasto público consolidado per cápita y no en la variación cross-section de los niveles de gasto entre las provincias.

Como es probable que, a pesar de los efectos fijos por provincia, por cohorte y por año, los controles por las características de padres e hijos así como por características socioeconómicas de las provincias incluidas en el modelo (2), existan factores inobservables omitidos que pueden segar el efecto estimado del gasto público sobre la movilidad intergeneracional, se implementa otra estrategia de identificación propuesta por ML. Esta técnica consiste en explorar el efecto del gasto público consolidado en la movilidad intergeneracional utilizando un enfoque de diferencias en diferencias. Específicamente, se asume que la "brecha de movilidad" entre hijos que

\footnotetext{
${ }^{143}$ La variable incluida indica para cada hijo adulto la proporción de años durante su adolescencia o juventud en los que el gobernador de su provincia fue del mismo partido que el presidente de turno. En la sección de 4 se presenta una breve justificación de su inclusión como variable de control.
} 
provienen de un trasfondo familiar aventajado y aquellos que no están en esta situación, es menor en las provincias con un elevado gasto público consolidado per cápita que en aquellas con un bajo gasto público. Entonces, si el GPCpc incrementa la movilidad global es probable que lo haga aumentando más la movilidad de los hijos más desventajados, en términos socioeconómicos, que la de aquellos más aventajadas dado que este es, o se espera que sea, uno de los principales objetivos del gasto público, particularmente del gasto público social. Para testear esta hipótesis se estima el siguiente modelo:

$$
\begin{aligned}
Y_{h i, c j t}= & \alpha+\beta Y_{p i, c j}+\delta_{1} E_{p i, c j}+\delta_{2}\left(Y_{p i, c j} \times E_{p, j c}\right)+\gamma A_{i c j}+\theta X_{c j} \\
& +\varphi D_{j}+\pi D_{t}+\vartheta\left(D_{t} \times D_{j}\right)+\lambda D_{c}+v_{c j}+\omega_{i c j t}
\end{aligned}
$$

donde $E_{p i, c j}$ es una variable dummy igual a uno si el nivel educativo de los padres es igual o menor al secundario completo - con esto se intenta identificar a los hijos que provienen de un trasfondo familiar desventajado o no -, $\omega_{c j t}$ es un término de error estocástico y $v_{c j}$ captura los factores omitidos inobservables que podrían sesgar los coeficientes estimados de interés $\delta_{1}$ y $\delta_{2}$, se asume que el efecto de estos factores inobservables es el mismo entre los hijos que experimentaron distintos niveles de gasto público per cápita en su provincia de residencia. Entonces, bajo este supuesto, al computar la diferencia en las diferencias del coeficiente $\delta_{2}$ se obtendrían estimaciones insesgadas del efecto del gasto público en las brechas de movilidad entre hijos de padres con baja y alta educación.

Como ya fue señalado en el capítulo anterior, un problema que se presenta para estimar los modelos (1), (2) ó (3) y, en general, la movilidad intergeneracional en países como Argentina es la falta de información de largo plazo para generaciones sucesivas de una misma familia. Una forma de solucionar este problema es implementado una estrategia de estimación con variables instrumentales utilizando dos muestras, una principal en la que se observa el ingreso de los hijos y ciertas características de los padres (educación, ocupación, ubicación geográfica) y una muestra auxiliar de un momento en el pasado en el que los padres eran más jóvenes. En base a la segunda muestra es posible predecir el ingreso de los padres en el pasado a partir de los parámetros estimados en la muestra auxiliar y de las características observadas de los padres en la muestra principal. Esos ingresos predichos son usados en la segunda etapa 
del procedimiento como regresores del modelo de ingresos de los hijos, estimado con la primera muestra $^{144}$.

En esta investigación, como en la mayoría de los estudios empíricos previos que analizan la movilidad intergeneracional del ingreso combinando dos conjuntos de datos diferentes, la elección de los instrumentos está limitada por la escasa información disponible. En función de ella, se emplea como variable instrumental la educación del padre $^{145}$. Sin embargo, de acuerdo con el análisis desarrollado en el capítulo anterior, si la educación del padre pertenece como regresor al modelo estructural del ingreso permanente del hijo, bajo supuestos plausibles ${ }^{146}$, esto ocasionaría una sobrestimación de la EII. En este caso, podría pensarse que nuestras estimaciones ofrecen un límite superior de la $\mathrm{EII}^{147}$.

En comparación con la MII, el análisis de la movilidad intergeneracional educativa presenta menos problemas de medición. En efecto, la educación tiene varias ventajas como variable de resultado sobre los ingresos en términos de estimación. Así, los individuos tienen a completar su educación a mediados de los 20 años entonces el análisis puede realizarse cuando los hijos son relativamente más jóvenes. En cambio, según el análisis del Haider y Solon (2006), para medir MII es conveniente medir el ingreso de padres e hijos en las edades centrales de su ciclo vital para evitar el denominado life-cycle bias $^{148}$. Asimismo, el error de medición es probablemente un problema menor cuando se utiliza la educación porque las personas informan con mayor precisión su educación (o la de sus padres) que sus ingresos. Además, una gran cantidad de estudios muestran que la educación está asociada positivamente con otros indicadores de bienestar socioeconómico como los ingresos, la salud y la esperanza de vida (Black y Deveraux, 2011).

\footnotetext{
${ }^{144}$ Para más detalles sobre ese método (conocido como TSIV), ver Capítulo 1.

${ }^{145}$ Numerosos estudios que intentan estimar la EII con el método TSIV, utilizaron la educación del padre como instrumento (Núñez y Risco, 2004; Núñez y Miranda, 2007; Lillard y Kilburn, 1995; Solon, 1992, entre otros).

${ }^{146}$ Estos supuestos son analizados formalmente en el Capítulo 2, véase sección 3.

${ }^{147}$ Pero, si en línea con los hallazgos Corcoran et al. (1992) y Mazumder (2005), el efecto directo de la educación del padre sobre el ingreso del hijo no es estadísticamente distinto de cero, una vez que se controla por el ingreso del padre promediado durante varios años, la estimación por IV y TSIV de la EII sería consistente. En el capítulo 1 se desarrolla una derivación formal del potencial sesgo presente en la EII estimada con estos métodos.

${ }^{148}$ Estas y otras cuestiones metodológicas relacionadas con la medición de la movilidad intergeneracional del ingreso se discuten formalmente en el Capítulo 2.
} 
Por otra parte, la estimación del grado de MI puede estar sesgada cuando se emplean, como en esta investigación, muestras de padres e hijos corresidentes ${ }^{149}$. La selección por corresidencia surge cuando se observan solamente los ingresos de hijos y padres que viven juntos y no se dispone de información sobre los padres que no residen con sus hijos (Nicoletti y Francesconi, 2006). Si la sub-muestra de individuos observados que residen con sus padres no es aleatoria, entonces la selección por corresidencia puede causar un sesgo en la estimación de la movilidad intergeneracional y llevar a una sub-representación de los ingresos reales de hijos adultos porque los que continúan viviendo con sus padres probablemente son aún estudiantes o no tienen ingresos suficientes para vivir independientemente. Si se asume que los hijos emancipados tienen, en promedio, mayores ingresos que los residentes con sus padres y provienen de una familia rica, la movilidad estimada sería mayor que la verdadera al no poder incluir estas familias en la muestra. Por el contrario, si aquellos provienen de una familia pobre, la movilidad intergeneracional computada sería menor que la verdadera. Un resultado similar resulta de suponer que los hijos emancipados son pobres. La dirección del sesgo no es, pues, tan clara (Sánchez Hugalde, 2004). No obstante, en el capítulo previo se compararon las estimaciones de la EII computadas para Argentina con una muestra de padres e hijos corresidentes, obtenidas de la EPH, con las derivadas utilizando una muestra libre de este sesgo, obtenida de la Encuesta de Percepción de Planes Sociales del 2007 (EPPS). Los resultados alcanzados con ambas muestras son similares, las estimaciones no presentan diferencias estadísticamente significativas y sugieren que este problema no las afecta considerablemente. Además, si este sesgo de selección es el mismo para los hijos corresidentes que experimentaron distintos niveles de gasto público en su niñez o adolescencia, entonces la técnica de diferencias en diferencias eliminaría su efecto del coeficiente de interés.

\footnotetext{
${ }^{149}$ Pocos estudios sobre movilidad intergeneracional analizan este sesgo de selección muestral, entre ellos se pueden mencionar los de Couch y Lillard (1998), Mazumder (2005), Nicoletti y Francesconi (2006) y Nicoletti (2008).
} 


\section{Datos}

Dado que el principal objetivo de esta investigación es determinar el efecto que tiene en la movilidad intergeneracional de ingresos, el gasto público las fuentes de información empleadas en esta investigación son varias. En primer lugar, se utilizaron los microdatos obtenidos de la Encuesta Permanente de Hogares (EPH) para el período 1998-2012. Por otro lado se emplearon los datos sobre el gasto público consolidado (GPC) distribuido por jurisdicción y desagregado por finalidad y función disponibles para el período 1998-2008 que provienen de la Dirección de Análisis del Gasto Público y Programas Sociales (DAGPyPS) del Ministerio de Economía y Finanzas Públicas de la Nación (MECON). El cómputo del GPC incluye las erogaciones del sector público no financiero correspondientes a los tres niveles de gobierno - nación, provincias y Ciudad Autónoma de Buenos Aires (CABA) y municipios. La consolidación es un método que permite mostrar las estadísticas de un conjunto de unidades (el gobierno nacional, provincial y municipal, en este caso) como si fuesen una sola, ya que elimina las transacciones y posiciones de saldo recíprocas entre las unidades (FMI, 2001). La elaboración de cuentas consolidadas involucra tres fases: homogeneización, eliminación y agregación. Homogeneizar la información implica abordar aspectos temporales, valorativos y terminológicos - como las clasificaciones presupuestarias - vinculados con las transacciones que realizan las unidades a consolidar. Por su parte, la segunda etapa comprende la eliminación de las transferencias que realiza un nivel de gobierno hacia otro, para evitar la doble contabilización. Por último, la agregación consiste en sumar las partidas, homogeneizadas y netas de transacciones (Jiménez, 2002). Los principales criterios metodológicos que emplea la Dirección de Análisis del Gasto Público y Programas Sociales (DAGPyPS) para consolidar el gasto son: i) imputar las erogaciones en el nivel de gobierno ejecutor y ii) utilizar la etapa presupuestaria devengado. El primer criterio hace referencia a que el gasto se imputa en el nivel de gobierno que lo ejecuta y no en el que lo financia. Por lo tanto, para evitar duplicaciones, las transferencias de fondos de Nación a provincias y municipios, así como la de provincias a municipios, se descuentan del nivel del gobierno financiador y son incluidas en el ejecutor. El criterio de base devengado alude al momento de registro de las transacciones incluidas en la consolidación. Es decir, los eventos económicos se registran en el período en que ocurren, independientemente de que se haya efectuado o 
de que esté pendiente el cobro o el pago efectivo ${ }^{150}$. Por otra parte, la distribución geográfica del GPC se realizó tomando como unidad de análisis el nivel de gobierno provincial, incluyendo la Ciudad de Buenos Aires. De este modo, sin tomar en cuenta las externalidades inter-jurisdiccionales, la distribución geográfica del GPC implica repartir entre las provincias las erogaciones, sujetas a consolidación, del gobierno nacional. El criterio utilizado para realizar la distribución geográfica del GPC es el criterio del beneficio. Es decir, se asigna el GPC según el lugar de residencia de los beneficiarios de las acciones del Estado. El procedimiento implementado para realizar esta distribución del GPC consiste en los siguientes pasos:

1. Análisis de los objetivos del programa presupuestario.

2. Imputación directa según ubicación geográfica de los beneficiarios a partir de la información obtenida por:

a. Consulta a la unidad ejecutora sobre información geográfica de las erogaciones vinculadas al programa que se contrasta con la información por ubicación geográfica del Sistema Integrado de Información Financiera (SIDIF). Si coincide se mantiene la distribución original del programa y si no coincide se modifica la distribución de acuerdo a la información provista por la unidad ejecutora del programa.

b. Consulta en la Cuenta Inversión (a) y por medio de las órdenes de pago libradas por la Tesorería General de la Nación (b) en caso de no obtener información de la unidad ejecutora.

3. Imputación indirecta a partir de distribuidores geográficos, si no se obtiene información concluyente en (a) y (b), como una aproximación de la distribución efectiva del gasto, en base a indicadores sectoriales y a los objetivos del programa presupuestario se utilizan.

También se utilizaron las series históricas de los siguientes indicadores provinciales como variables de control:

- Los recursos tributarios provinciales cuya información se obtuvo de la Dirección de Coordinación Fiscal con las provincias del MECON.

\footnotetext{
${ }^{150}$ Para mayor detalle sobre la metodología de consolidación del gasto público del sector público no financiero, ver DAGPyPS (2011).
} 
- La carga tributaria nacional por jurisdicción (proporción de la recaudación nacional que se obtiene de cada provincia y la Ciudad Autónoma de Buenos Aires) obtenida de los estudios de Zapata et al. (2000), Sanguinetti y Tommasi (2001) y Müller y Rico (2010).

- La tasa de pobreza por Necesidades básicas insatisfechas (NBI), la tasa de desocupación, el coeficiente de Gini, la tasa de asistencia a cada nivel educativo de la población en el grupo etario objetivo, los años promedios de educación de la PET, la proporción de la población sin cobertura de salud, el porcentaje de la población sin acceso a agua corriente y sin acceso a desagües cloacales por provincia. Todos estos indicadores fueron computados a partir de la EPH.

- La tasa de mortalidad infantil (por mil nacidos vivos) y la tasa de mortalidad total (por mil habitantes) por provincia obtenidas de la Dirección de Estadística e Información de Salud (DEIS) del Ministerio de Salud de la Nación.

- La población total por provincia que se obtuvo de las proyecciones de población realizada por el Instituto Nacional de Estadísticas y Censos (INDEC, 2005).

- La tasa de crecimiento del PBG per cápita de cada provincia computada a partir de la información obtenida de las Direcciones Provinciales de Estadística.

- El partido político del gobernador de cada provincia en relación con el del presidente de turno, información sistematizada por la Unidad de Información Provincial Integrada (ProvInfo) de la Secretaría de Provincias del Ministerio del Interior y Transporte. La variable construida a partir de esta información indica para cada hijo adulto la proporción de años durante su adolescencia o juventud en los que el gobernador de su provincia fue del mismo partido que el presidente de turno. La inclusión de esta variable como control en el modelo empírico se fundamenta principalmente en la evidencia empírica disponible que revela que más allá de las necesidades y capacidades fiscales locales, otros factores relacionados con las influencias políticas juegan un rol importante en la determinación de las asignaciones horizontales de las transferencias intergubernamentales (Boex y Martinez-Vazquez, 2005; Porto y Sanguinetti, 2001, Atlas et al., 1995).

La muestra principal incluye a hijos adultos de 25 a 34 años que nacieron entre 1978 y 1987 con ingresos familiares per cápita positivos y proviene de la EPH en su 
modalidad continua para el período 2003-2012 $2^{151}$. La elección del rango etario para los hijos así como del año inicial del período analizado responde a varias razones, entre ellas, las limitaciones informativas relacionadas con la falta de disponibilidad de datos del gasto público consolidado provincial por finalidad y función para años previos a 1998 y el método de estimación implementado para estimar el ingreso paterno en la adolescencia o juventud de los hijos. Como la EPH no contiene información sobre el ingreso de los padres cuando los hijos eran más jóvenes ${ }^{152}$, a fin de predecir el ingreso familiar en la adolescencia de los hijos se emplea otra muestra anterior obtenida de los datos de la EPH relevada en un año previo al de la muestra principal, comprendido en el período 1998-2008, según la cohorte de nacimiento de cada hijo. La elección de estos años - así como del rango etario para los hijos - obedece a varias razones. En primer lugar, se asume - siguiendo a Núñez y Miranda (2007) y Núñez y Risco (2004) - que los padres toman las principales decisiones de inversiones en el capital humano de sus hijos cuando éstos son niños y adolescentes ${ }^{153}$. Estas inversiones constituyen una de las principales fuentes de transmisión socioeconómica entre generaciones. Se utiliza como medida de los recursos familiares disponibles para realizar estas inversiones un promedio del ingreso familiar per cápita paterno estimado cuando los hijos tenían menos de 24 años ${ }^{154}$. Los individuos que tienen entre 25 y 34 años en cada año del período 2003-2012, tenían 24 años o menos, en algún año del período 1998-2008, según su cohorte de nacimiento (ver Cuadro A1). En segundo lugar, la ampliación del rango de edad de los hijos implicaría emplear datos de la EPH de años anteriores al considerado para cada cohorte a fin de predecir el ingreso paterno en su adolescencia y

\footnotetext{
${ }^{151}$ Para obtener una muestra anual de hijos adultos a partir de cada EPH, se unieron las observaciones correspondientes a los distintos relevamientos realizados dentro del año (dos, en la EPHP y cuatro, en la EPHC) pero dado que la encuesta tiene la estructura de un panel corto de datos y en cada relevamiento la muestra se renueva en un determinado porcentaje, se eliminaron las observaciones repetidas, dejando una observación para cada individuo en cada año a fin de no sesgar los resultados.

${ }^{152} \mathrm{La}$ encuesta no incluye preguntas retrospectivas sobre el ingreso de los padres en un momento en el pasado del ciclo de vida de los hijos y tiene la estructura de un panel corto de datos.

${ }^{153}$ Behrman y Taubman (1990) reportan que la persistencia de ingresos es mayor cuando los padres son observados durante los años de escolaridad de los hijos antes que en un momento posterior de su ciclo de vida.

${ }^{154}$ Así, por ejemplo, para estimar el ingreso promedio del padre para la cohorte de hijos adultos del año 1984 que tiene 28 años en 2012 se utilizaron las muestras secundarias obtenidas de la EPH para el período 1999 a 2008, años en los que los hijos de la muestra principal tenían entre 15 y 24 años. Estas muestras secundarias están conformada por individuos adultos varones que tienen de 25 a 55 años y además son padres de hijos de la misma cohorte de nacimiento que los hijos adultos de la muestra principal. A partir de estas muestras auxiliares se estiman los retornos de la educación de los padres en cada año del período 1998-2008. Estos retornos se utilizan luego para computar el ingreso predicho que el padre de cada hijo adulto de la muestra principal obtenía, en promedio, en función de su educación.
} 
juventud. Pero, en este caso, la cantidad de aglomerados cubiertos por la encuesta se reduciría $^{155}$.

Por otro lado, los datos sobre el gasto público consolidado (GPC) por provincia y por finalidad y función sólo están disponibles desde 1998. A fin de utilizar una medida de la inversión pública en el capital humano de los hijos durante el mismo período en que se mide la inversión privada realizada por los padres en sus hijos y en la misma etapa del ciclo de vida para todas las cohortes, se utiliza como proxy de esa inversión pública, el gasto público consolidado per cápita ${ }^{156}$ (GPCpc) promediado cuando los adultos de la muestra principal tenían entre 18 y 24 años ${ }^{157}$ (ver Cuadro A1). Como advierten ML, como indicador de inversión, el gasto público comparte las mismas limitaciones que el ingreso familiar como proxy de la inversión parental. Así, las provincias que gastan la misma cantidad podrían comprar diferentes bienes y tener diferentes niveles de eficiencia en sus compras dependiendo de una variedad de circunstancias. No obstante, algunas categorías del GPC como el gasto social, el gasto en educación, en atención de la salud, cuyos efectos individuales sobre la movilidad intergeneracional se examinan en esta investigación, podrían considerarse como medidas menos imperfectas de la inversión pública en distintos aspectos configurativos del capital humano de los niños y adolescentes que habitan cada provincia.

Debe advertirse también que, como la EPH recolecta información sólo de los centros urbanos más importantes del país en términos de tamaño de población, las muestras son representativas de las ciudades pero no de toda la población nacional. Esto puede llevar a una sobrestimación de la movilidad generacional en el país porque la muestra considerada no representa grupos de la población para quienes se espera que la persistencia generacional de ingresos sea mayor, tales como aquellos que viven en áreas

\footnotetext{
${ }^{155}$ Los aglomerados cubiertos por las EPH desde 1998 hasta el 2012 (en una o ambas ondas de la EPH en su modalidad puntual) son 29. Por lo tanto, se cuenta con al menos un aglomerado de todas las provincias argentinas, con excepción de Río Negro (cuyo aglomerado Viedma-Carmen de Patagones se incorporó a la EPH en 2002).

${ }^{156}$ El gasto público per cápita se computa como el cociente entre el gasto público consolidado correspondiente a cada provincia y la población provincial total en cada año.

${ }^{157}$ Debe advertirse que se asume, por falta de información, que los hijos adultos de la muestra principal residen en la misma provincia en que habitaron durante su juventud. Claramente, este supuesto no se cumple en caso de los que migraron después y no retornaron a la provincia en la que vivieron entre los 18 y 24 años. Sin embargo, según los datos de la EPH, el porcentaje promedio de los individuos de la muestra que nacieron en otra provincia o país es $8.7 \%$ y el porcentaje promedio que realizó una migración internacional y/o entre provincias en los últimos 5 años es $0.6 \%$. Y, la eliminación de estos individuos de la muestra no modifica sustancialmente los resultados obtenidos.
} 
rurales o pequeños centros urbanos (Núñez y Miranda, 2007). No obstante, dado que en Argentina un elevado porcentaje de la población total habita en centros urbanos, por lo que actualmente, la EPH representa aproximadamente el 70\% de la población urbana y el $60 \%$ de la población total del país.

En el Cuadro 1 se reportan algunas características de las muestras de hijos utilizadas en el análisis empírico. La primera columna corresponde a la muestra total de hijos de 25 a 34 años que habitaban con sus padres en algún año del período 2003-2012. En las columnas siguientes se observan las características para las tres muestras de hijos construidas según el nivel promedio de gasto público consolidado que experimentaron durante su adolescencia y juventud. La clasificación de los niveles de gasto en bajo, medio y alto surge de dividir en tres partes la distribución del gasto público consolidado per cápita promedio en la juventud de cada hijo adulto incluido en la muestra principal. En el Cuadro 1, así como en el análisis empírico siguiente, las estimaciones se realizaron considerando, además del gasto público consolidado per cápita (GPCpc) total, distintas categorías y subcategorías del gasto público social consolidado per cápita (GPSCpc): el gasto público en educación total, en educación básica, en educación superior, en cultura, en salud total, en atención de la salud y obras sociales ${ }^{158}$, en agua potable y alcantarillado, vivienda y urbanismo, en promoción y asistencia social, en previsión social y en programas de empleo.

\footnotetext{
${ }^{158}$ La incorporación de la categoría "obras sociales" como Gasto Público merece una aclaración metodológica. La DAGPyPS del MECON justifica su inclusión en varias razones, entre ellas, por considerar que su figura jurídica, creada por ley especial de la Nación, no se rige por la legislación general para personas jurídicas de carácter civil o comercial. Además, las obras sociales son entidades que proporcionan servicios de salud, turismo y asistencia social a sus afiliados y son financiadas con impuestos sobre la nómina salarial, contribuciones patronales y aportes de los trabajadores. Asimismo, debe destacarse el carácter solidario del seguro. Por último, la contabilización de este seguro social como gasto público es convalidada por la metodología internacional, típicamente la del Fondo Monetario Internacional (DAGPyPS, 2009).
} 
Cuadro 1. Características de las muestras utilizadas en el análisis

\begin{tabular}{|c|c|c|c|c|c|c|c|c|}
\hline \multirow{3}{*}{$\begin{array}{l}\text { Características } \\
\text { Edad }\end{array}$} & \multirow{2}{*}{\multicolumn{2}{|c|}{ Todos los hijos }} & \multicolumn{6}{|c|}{ Hijos según nivel de gasto público consolidado en su juventud } \\
\hline & & & \multicolumn{2}{|c|}{ Bajo } & \multicolumn{2}{|c|}{ Medio } & \multicolumn{2}{|c|}{ Alto } \\
\hline & 27.22 & $(2.17)$ & 27.28 & $(1.97)$ & 27.48 & $(2.36)$ & 26.85 & $(2.10)$ \\
\hline Edad promedio del padre en la juventud del hijo & 50.67 & $(7.17)$ & 50.28 & $(7.19)$ & 50.89 & $(7.36)$ & 50.86 & $(6.88)$ \\
\hline Varón & 0.54 & $(0.50)$ & 0.55 & $(0.50)$ & 0.54 & $(0.50)$ & 0.55 & $(0.50)$ \\
\hline \multicolumn{9}{|l|}{ Nivel educativo } \\
\hline Primaria incompleta & 0.03 & $(0.18)$ & 0.03 & $(0.18)$ & 0.04 & $(0.19)$ & 0.03 & $(0.16)$ \\
\hline Primaria completa & 0.10 & $(0.30)$ & 0.12 & $(0.33)$ & 0.12 & $(0.32)$ & 0.06 & $(0.24)$ \\
\hline Secundaria incompleta & 0.14 & $(0.35)$ & 0.15 & $(0.35)$ & 0.15 & $(0.36)$ & 0.13 & $(0.33)$ \\
\hline Secundaria completa & 0.27 & $(0.45)$ & 0.28 & $(0.45)$ & 0.28 & $(0.45)$ & 0.26 & $(0.44)$ \\
\hline Universitaria incompleta & 0.27 & $(0.45)$ & 0.26 & $(0.44)$ & 0.25 & $(0.43)$ & 0.32 & $(0.47)$ \\
\hline Universitaria completa & 0.18 & $(0.38)$ & 0.16 & $(0.37)$ & 0.17 & $(0.37)$ & 0.21 & $(0.41)$ \\
\hline \multicolumn{9}{|l|}{ Nivel educativo del padre } \\
\hline Primaria incompleta & 0.14 & $(0.34)$ & 0.16 & $(0.36)$ & 0.15 & $(0.35)$ & 0.10 & $(0.30)$ \\
\hline Primaria completa & 0.33 & $(0.47)$ & 0.35 & $(0.48)$ & 0.37 & $(0.48)$ & 0.27 & $(0.45)$ \\
\hline Secundaria incompleta & 0.14 & $(0.35)$ & 0.14 & $(0.35)$ & 0.14 & $(0.35)$ & 0.14 & $(0.35)$ \\
\hline Secundaria completa & 0.19 & $(0.39)$ & 0.19 & $(0.39)$ & 0.18 & $(0.38)$ & 0.20 & $(0.40)$ \\
\hline Universitaria incompleta & 0.06 & $(0.24)$ & 0.06 & $(0.23)$ & 0.05 & $(0.22)$ & 0.08 & $(0.28)$ \\
\hline Universitaria completa & 0.13 & $(0.34)$ & 0.10 & $(0.30)$ & 0.11 & $(0.31)$ & 0.20 & $(0.40)$ \\
\hline Ingreso familiar per cápita ( $\$$ de 2001) & 402.59 & $(346.28)$ & 360.67 & $(293.48)$ & 367.79 & $(317.87)$ & 489.20 & $(410.15)$ \\
\hline $\begin{array}{l}\text { Ingreso familiar per cápita estimado del padre } \\
\text { (\$ de 2001) }\end{array}$ & 170.73 & $(100.67)$ & 153.84 & $(89.23)$ & 163.11 & $(97.12)$ & 198.18 & $(110.51)$ \\
\hline \multicolumn{9}{|l|}{ Gasto público consolidado per cápita ( $\$$ de } \\
\hline \multicolumn{9}{|l|}{ 2001) prome dio durante los 18 a 24 años } \\
\hline Total & 2291.33 & $(582.80)$ & 1917.89 & $(58.83)$ & 2099.41 & $(58.95)$ & 2925.42 & (708.76) \\
\hline Servicios Sociales & 1495.27 & $(373.01)$ & 1250.20 & $(96.05)$ & 1386.91 & $(69.21)$ & 1890.94 & $(449.56)$ \\
\hline GPSCH1 & 765.14 & $(207.63)$ & 634.07 & $(40.12)$ & 704.02 & $(31.57)$ & 980.41 & $(261.11)$ \\
\hline GPSCH2 & 800.21 & $(225.96)$ & 661.13 & $(29.38)$ & 729.58 & $(31.30)$ & 1035.40 & $(286.61)$ \\
\hline GPSCH3 & 749.62 & $(201.12)$ & 622.38 & $(39.20)$ & 690.34 & $(28.52)$ & 958.57 & $(252.61)$ \\
\hline GPSCH4 & 686.13 & $(179.49)$ & 573.96 & $(38.48)$ & 632.60 & $(28.57)$ & 871.82 & $(225.63)$ \\
\hline Salud & 345.37 & $(109.86)$ & 284.33 & $(29.31)$ & 313.54 & $(22.56)$ & 449.54 & $(147.40)$ \\
\hline Atención médica & 140.93 & $(61.05)$ & 111.57 & $(13.23)$ & 119.38 & $(13.98)$ & 198.30 & $(83.44)$ \\
\hline Obras sociales & 150.88 & $(45.73)$ & 125.86 & $(12.72)$ & 137.50 & $(10.73)$ & 193.97 & $(61.31)$ \\
\hline Promoción y Asistencia Social & 84.35 & $(29.80)$ & 66.97 & $(7.37)$ & 76.65 & $(9.82)$ & 112.42 & $(39.21)$ \\
\hline Promoción y asistencia social pública & 41.50 & $(21.03)$ & 31.69 & $(5.10)$ & 37.63 & $(6.64)$ & 56.78 & (31.72) \\
\hline Nutrición y alimentación & 20.86 & $(7.76)$ & 18.55 & $(3.80)$ & 18.91 & $(4.17)$ & 25.66 & (11.27) \\
\hline Deporte y recreación & 2.54 & $(3.15)$ & 1.20 & $(0.44)$ & 1.70 & $(0.59)$ & 5.00 & $(4.79)$ \\
\hline Obras sociales & 8.52 & (3.69) & 6.80 & $(1.06)$ & 7.39 & $(0.89)$ & 11.73 & $(5.21)$ \\
\hline Seguridad Social & 534.11 & $(153.68)$ & 443.32 & $(57.25)$ & 504.29 & $(46.04)$ & 668.68 & (206.88) \\
\hline Educación y Cultura & 319.91 & $(76.68)$ & 271.08 & $(17.13)$ & 300.15 & $(13.31)$ & 396.61 & $(98.50)$ \\
\hline Educación básica & 213.84 & $(58.76)$ & 181.63 & $(14.83)$ & 202.33 & $(16.49)$ & 262.67 & $(83.84)$ \\
\hline Educación elemental & 135.31 & (39.68) & 116.14 & $(11.58)$ & 127.35 & $(10.68)$ & 165.66 & $(58.81)$ \\
\hline Educación media y técnica & 78.53 & (21.98) & 65.49 & $(10.66)$ & 74.98 & $(9.10)$ & 97.01 & $(28.51)$ \\
\hline Educación superior y universitaria & 66.34 & $(20.93)$ & 55.41 & $(8.83)$ & 62.12 & $(8.02)$ & 83.28 & $(28.80)$ \\
\hline Cultura & 13.22 & $(13.80)$ & 6.73 & $(1.53)$ & 8.71 & $(2.86)$ & 25.60 & $(19.65)$ \\
\hline Ciencia y Técnica & 14.85 & $(2.67)$ & 13.51 & $(1.57)$ & 14.75 & $(1.48)$ & 16.43 & $(3.68)$ \\
\hline Trabajo & 81.92 & $(22.16)$ & 74.74 & $(8.40)$ & 74.02 & $(11.81)$ & 99.03 & $(30.68)$ \\
\hline Programas de empleo y seguro de desempleo & 41.87 & $(16.41)$ & 45.00 & $(8.09)$ & 39.86 & $(9.25)$ & 40.77 & $(26.28)$ \\
\hline Asignaciones familiares & 40.05 & $(21.97)$ & 29.74 & $(6.57)$ & 34.15 & (7.63) & 58.26 & (31.16) \\
\hline Vivienda y Urbanis mo & 35.08 & $(35.38)$ & 27.05 & $(15.49)$ & 25.55 & $(14.25)$ & 54.99 & $(54.88)$ \\
\hline Agua Potable y Alcantarillado & 15.51 & (14.48) & 11.70 & $(3.40)$ & 13.69 & $(5.73)$ & 21.84 & $(23.96)$ \\
\hline Otros Servicios Urbanos & 64.18 & $(23.95)$ & 57.50 & $(16.73)$ & 64.28 & $(14.44)$ & 71.41 & $(34.94)$ \\
\hline Observaciones (sin ponderar) & \multicolumn{2}{|c|}{16119} & \multicolumn{2}{|c|}{4759} & \multicolumn{2}{|c|}{4235} & \multicolumn{2}{|c|}{7125} \\
\hline
\end{tabular}

Fuente: Elaboración propia sobre la base de EPH, INDEC y DAGPyPS, MECON.

Nota: Entre paréntesis se reportan los desvíos estándares. El gasto público se expresa en pesos constantes de 2001 utilizando un promedio del Índice de Precios al Consumidor y del Índice de Precios Mayorista.

También se consideran agregados del GPSCpc que pueden tener un efecto más directo en el capital humano de hijos (GPSCH, en adelante). Estas medidas se diferencian entre sí por las categorías de GPSCpc incluidas en cada una. Así, la primera 
medida (GPSCH1) es la más amplia e incluye el GPSCpc en salud, promoción y asistencia social, educación total, agua y alcantarillado y vivienda, la segunda (GPSCH2) surge de restar a la anterior el gasto público en vivienda que podría considerarse un categoría de gasto que no beneficia directamente a niños y jóvenes, la tercera (GPSCH3) incluye el GPSCpc en salud, promoción y asistencia social y educación total. Finalmente, la última medida utilizada (GPSCH4) surge de agregar las categorías de gasto social que se consideran más específicas y dirigidas a incrementar el capital humano de niños y jóvenes: salud, nutrición ${ }^{159}$ y educación.

\section{Resultados}

\subsection{Efecto de distintas medidas de inversión pública en capital humano en la movilidad intergeneracional}

En el Cuadro 2 se observan las elasticidades intergeneracionales del ingreso y los coeficientes de persistencia educativa obtenidos a partir de la estimación de distintas especificaciones del modelo (1) utilizando el método TSIV en el caso de la EII y el método de OLS para el CPE.

Cuadro 2. Medidas de persistencia intergeneracional. 2003- 2012

\begin{tabular}{ccccc}
\hline \hline Medida & Modelo 1 & Modelo 2 & Modelo 3 & Modelo 4 \\
\hline Elasticidad & $0.701 * * *$ & $0.648 * * *$ & $0.648 * * *$ & $0.648 * * *$ \\
intergeneracional del & $(0.041)$ & $(0.015)$ & $(0.015)$ & $(0.015)$ \\
Coeficiente de Persistencia & $0.392 * * *$ & $0.382 * * *$ & $0.379 * * *$ & $0.379 * * *$ \\
educativa & $(0.009)$ & $(0.015)$ & $(0.015)$ & $(0.015)$ \\
\hline
\end{tabular}

Fuente: Elaboración propia sobre la base de EPH, INDEC y DAGPyPS, MECON.

Nota: $* * * p$-value $<0.01$, ** p-value $<0.05, * p$-value $<0.1$. Entre paréntesis se reportan los errores estándares agrupados a nivel provincial. El Modelo 1 incluye además del logaritmo del ingreso predicho del padre, controles por el sexo del hijo, la edad del hijo y de los padres (polinomio cuártico); el Modelo 2, incluye además de las variables anteriores, efectos fijos por provincia de residencia, por cohorte de nacimiento y por año; en el Modelo 3, se agregan los términos de interacciones entre las dummies por provincia y las temporales y en el Modelo 4, se incluye el gasto público consolidado per cápita total.

\footnotetext{
${ }^{159}$ Cabe aclarar que el gasto público en nutrición incluye programas que benefician a la población de cualquier franja etaria y no solamente a niños en edad escolar.
} 
Los índices de persistencia socioeconómica intergeneracional son estadísticamente significativos a un nivel del $1 \%$ y no presentan variaciones considerables entre las distintas especificaciones. La EII promedio a nivel nacional para el período 2003-2012 e hijos de 25 a 39 años, varía de 0.65 a 0.70 , mientras que el CPE tiene valores entre 0.38 y 0.39 según los controles incluidos en el modelo estimado. Conforme a los modelos teóricos mencionados en la sección 2, se espera que estos índices de persistencia intergeneracional varíen con los niveles de gastos público que experimentaron los hijos cuando eran adolescentes o jóvenes. Como primera exploración empírica de esta hipótesis, se reportan en el Gráfico 1 los CPE estimados a partir del modelo intergeneracional básico (1), que relaciona los años de educación de los hijos con el de sus padres, para tres niveles ${ }^{160}$ de las distintas medidas consideradas de gasto público. En general, se aprecia un aumento significativo en esta medida de inmovilidad intergeneracional cuando el nivel promedio de gasto per cápita experimentado por los hijos en su juventud disminuye de alto a bajo.

\section{Gráfico 1. CPE estimado a partir del modelo intergeneracional básico para tres niveles de gasto}

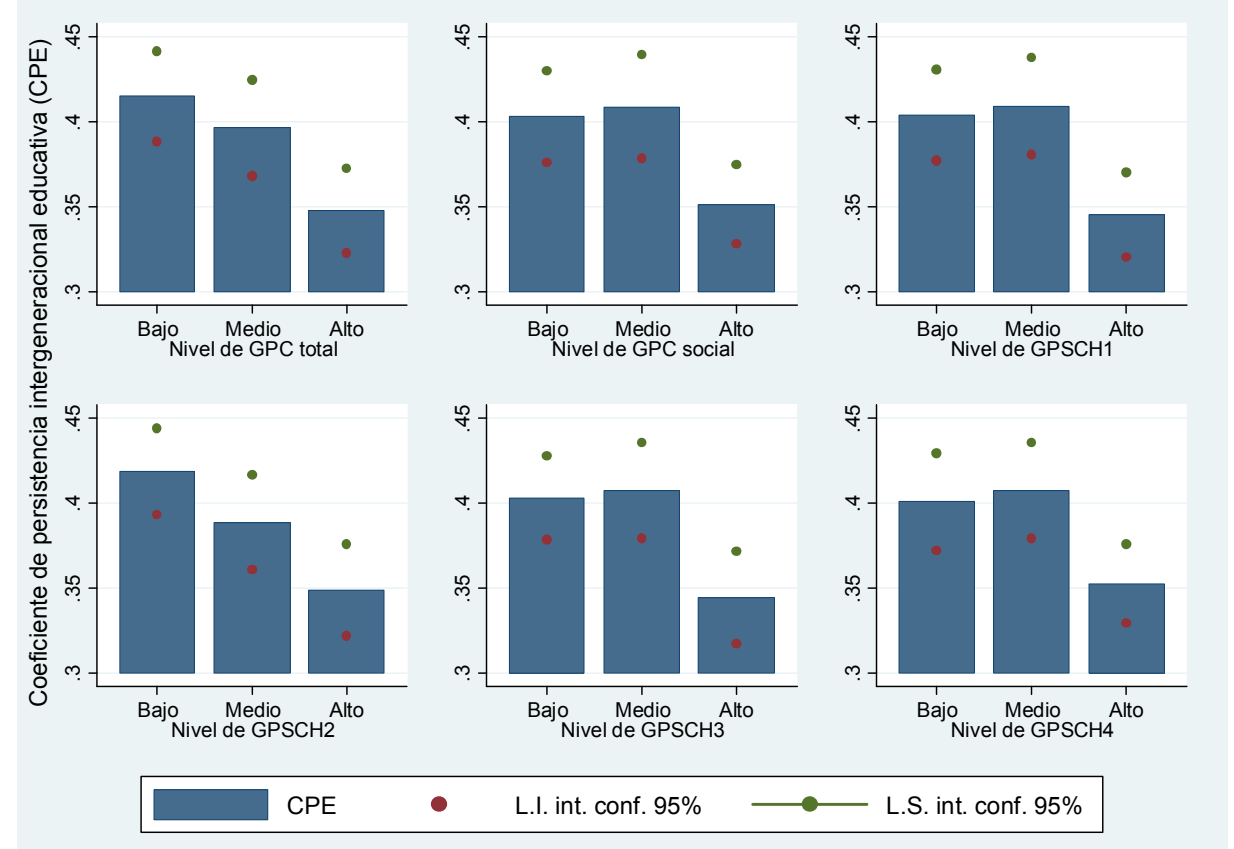

Fuente: Elaboración propia sobre la base de EPH, INDEC y DAGPyPS, MECON.

Nota: El modelo utilizado para estimar el CPE que relaciona la educación de padres e hijos no incluye ninguna variable de control.

\footnotetext{
${ }^{160}$ La clasificación de los niveles de gasto en bajo, medio y alto surge de dividir en terciles la distribución del gasto público consolidado per cápita promedio en la juventud de cada hijo adulto incluido en la muestra principal.
} 


\section{Cuadro 3. Efecto del gasto público consolidado per cápita sobre la elasticidad intergeneracional del ingreso}

\begin{tabular}{|c|c|c|c|c|}
\hline $\begin{array}{l}\text { Medidas } \\
\text { GPCpc }\end{array}$ & Variables de interés & Mod. 1 & Mod. 2 & Mod. 3 \\
\hline Total & $\begin{array}{l}\text { Log del ingreso del padre } \\
\text { Log del Gasto público per cápita } \\
\text { Log del ingreso del padre Log del Gasto público per } \\
\text { cápita }\end{array}$ & $\begin{array}{c}1.793 * * * \\
(0.296) \\
0.291 * \\
(0.157) \\
-0.148 * * * \\
(0.037)\end{array}$ & $\begin{array}{c}1.792 * * * \\
(0.295) \\
0.674 * \\
(0.384) \\
-0.148 * * * \\
(0.037)\end{array}$ & $\begin{array}{c}1.987 * * * \\
(0.274) \\
1.152 * * * \\
(0.374) \\
-0.174 * * * \\
(0.035)\end{array}$ \\
\hline $\begin{array}{l}\text { Servicios } \\
\text { Sociales }\end{array}$ & $\begin{array}{l}\text { Log del ingreso del padre } \\
\text { Log del Gasto público per cápita } \\
\text { Log del ingreso del padre Log del Gasto público per } \\
\text { cápita }\end{array}$ & $\begin{array}{c}1.715 * * * \\
(0.253) \\
0.311 * \\
(0.151) \\
-0.147 * * * \\
(0.034) \\
\end{array}$ & $\begin{array}{c}1.704 * * * \\
(0.255) \\
0.381 \\
(0.434) \\
-0.145 * * * \\
(0.034) \\
\end{array}$ & $\begin{array}{c}1.879 * * * \\
(0.224) \\
0.626 \\
(0.434) \\
-0.169 * * * \\
(0.030) \\
\end{array}$ \\
\hline GPSCH1 & $\begin{array}{l}\text { Log del ingreso del padre } \\
\text { Log del Gasto público per cápita } \\
\text { Log del ingreso del padre Log del Gasto público per } \\
\text { cápita }\end{array}$ & $\begin{array}{c}1.580 * * * \\
(0.238) \\
0.294 * \\
(0.167) \\
-0.140 * * * \\
(0.035)\end{array}$ & $\begin{array}{c}1.583 * * * \\
(0.233) \\
0.871 * * \\
(0.415) \\
-0.141 * * * \\
(0.034)\end{array}$ & $\begin{array}{c}1.714 * * * \\
(0.228) \\
1.011 * * \\
(0.436) \\
-0.160 * * * \\
(0.033)\end{array}$ \\
\hline GPSCH2 & $\begin{array}{l}\text { Log del ingreso del padre } \\
\text { Log del Gasto público per cápita } \\
\text { Log del ingreso del padre Log del Gasto público per } \\
\text { cápita }\end{array}$ & $\begin{array}{c}1.615 * * * \\
(0.232) \\
0.308 * \\
(0.156) \\
-0.146 * * * \\
(0.034) \\
\end{array}$ & $\begin{array}{c}1.618 * * * \\
(0.230) \\
0.508 \\
(0.441) \\
-0.147 * * * \\
(0.034) \\
\end{array}$ & $\begin{array}{c}1.758 * * * \\
(0.210) \\
0.959 * * \\
(0.419) \\
-0.168 * * * \\
(0.031) \\
\end{array}$ \\
\hline GPSCH3 & $\begin{array}{l}\text { Log del ingreso del padre } \\
\text { Log del Gasto público per cápita } \\
\text { Log del ingreso del padre Log del Gasto público per } \\
\text { cápita }\end{array}$ & $\begin{array}{c}1.618 * * * \\
(0.238) \\
0.308 * \\
(0.161) \\
-0.147 * * * \\
(0.035)\end{array}$ & $\begin{array}{c}1.628 * * * \\
(0.235) \\
0.660 \\
(0.435) \\
-0.149 * * * \\
(0.035)\end{array}$ & $\begin{array}{c}1.770 * * * \\
(0.206) \\
1.010 * * \\
(0.386) \\
-0.170 * * * \\
(0.030)\end{array}$ \\
\hline GPSCH4 & $\begin{array}{l}\text { Log del ingreso del padre } \\
\text { Log del Gasto público per cápita } \\
\text { Log del ingreso del padre Log del Gasto público per } \\
\text { cápita }\end{array}$ & $\begin{array}{c}1.619 * * * \\
(0.246) \\
0.303 * \\
(0.170) \\
-0.149 * * * \\
(0.037)\end{array}$ & $\begin{array}{c}1.629 * * * \\
(0.247) \\
0.854 * * \\
(0.391) \\
-0.151 * * * \\
(0.037)\end{array}$ & $\begin{array}{c}1.772 * * * \\
(0.211) \\
1.192 * * * \\
(0.373) \\
-0.173 * * * \\
(0.031)\end{array}$ \\
\hline
\end{tabular}

Fuente: Elaboración propia sobre la base de varias fuentes de información. Ver la sección de datos para más detalles.

Nota: $* * * p$-value $<0.01, * * p$-value $<0.05, * p$-value $<0.1$. Entre paréntesis se reportan los errores estándares agrupados a nivel provincial. El Modelo 1 incluye además de las variables de interés, controles por el sexo del hijo, la edad del hijo y de los padres (polinomio cuártico), efectos fijos por provincia de residencia, dummies por año, el Modelo 2 suma a las anteriores dummies por cohorte y un conjunto de variables de control por características provinciales promedios existentes durante la juventud de los hijos (los recursos tributarios provinciales, la carga tributaria nacional por provincia, la tasa de desocupación, el nivel de desigualdad, la tasa de crecimiento del PBG, la tasa de pobreza por NBI, la tasa de asistencia a cada nivel educativo, los años de educación promedio de la PET, la cantidad total de población, la proporción de la población sin cobertura de salud, la tasa de mortalidad infantil (por mil nacidos vivos), la tasa de mortalidad total (por mil habitantes), el porcentaje de la población sin acceso a agua corriente y sin acceso a desagües cloacales, el partido político del gobernador de cada provincia en relación con el del presidente de turno) y la proporción del gasto público per cápita destinado a otras partidas, salvo en el modelo estimado para el GPCpc total y el Modelo 3, incorpora al anterior los términos de interacción entre las dummies locales y temporales.

En el Cuadro 3 se reportan los resultados de la estimación de distintas especificaciones del modelo intergeneracional del ingreso (2) que incluye además del logaritmo del ingreso predicho del padre y del gasto público promedio en la juventud 
del hijo, un término de interacción entre esas dos variables que permite examinar el efecto de un mayor nivel de GPCpc en la persistencia intergeneracional del ingreso medida por la EII (el coeficiente del log del ingreso paterno). En los paneles 3 a 5 del cuadro se presentan los resultados obtenidos al considerar distintas medidas del GPSCpc destinado al capital humano de hijos (GPSCH).

En todas las especificaciones el ingreso estimado del padre tiene un efecto positivo y estadísticamente significativo al 1\% en el ingreso del hijo. Por su parte, todas las medidas de gasto público consideradas presentan un efecto positivo en el ingreso de los hijos en todos las especificaciones, que es estadísticamente significativo en la mayoría de los casos, particularmente en el modelo más completo que incluye términos de interacción entre los efectos fijos locales y temporales y controles por ciertas características provinciales como, por ejemplo, la tasa de pobreza por NBI, la tasa de desocupación, la desigualdad (medida con el coeficiente de Gini), la tasa de mortalidad infantil, que están correlacionadas positivamente con algunas categorías del gasto público pero negativamente con el ingreso de los hijos. Según Harknett (2003) la evidencia empírica disponible sobre la relación entre gasto y resultados de los hijos es mixta. Es decir, a partir de los estudios disponibles no se concluye claramente si el gasto público tiene un efecto positivo en los resultados de los hijos. Además, las inversiones privadas realizadas por los padres no sólo en dinero, sino también en tiempo y otros factores ambientales afectan el bienestar de los hijos. Si estos factores varían entre cohortes podrían disminuir o, incluso, eliminar el efecto directo del gasto público.

Los términos de interacción entre el GPCpc y el ingreso del padre tienen el signo esperado, siendo negativos y estadísticamente significativos al 1\% para todas las medidas de inversión pública en capital humano consideradas. En principio, estos resultados indican que a mayores niveles de inversión pública en capital humano experimentados en la juventud, menor es la persistencia intergeneracional de ingresos medida por la EII.

Cuando se consideran las estimaciones para el modelo intergeneracional educativo, reportadas en el Cuadro 4, la educación del padre tiene en todos los modelos estimados un efecto positivo y estadísticamente significativo a un nivel de significancia del 1\%. Los coeficientes del GPCpc presentan también los signos positivos esperados y resultan estadísticamente significativos en la mayoría de las medidas consideradas 
particularmente en la especificación más completa (modelo 3). Los términos de interacción entre el GPCpc y la educación del padre, por su parte, tienen en todas las medidas de gasto analizadas, el signo negativo esperado, siendo además estadísticamente significativos, en los tres modelos estimados.

\section{Cuadro 4. Efecto del gasto público consolidado per cápita sobre el coeficiente de persistencia educativa intergeneracional}

\begin{tabular}{|c|c|c|c|c|}
\hline \begin{tabular}{|l|} 
Medidas \\
GPCpc \\
\end{tabular} & Variables de interés & Mod. 1 & Mod. 2 & Mod. 3 \\
\hline Total & $\begin{array}{l}\text { Educación del padre } \\
\text { Log Gasto público per cápita } \\
\begin{array}{l}\text { Educación del padre x Log Gasto público per } \\
\text { cápita }\end{array}\end{array}$ & $\begin{array}{c}1.660 * * * \\
(0.467) \\
1.778^{*} \\
(1.033) \\
-0.166^{* *} \\
(0.061)\end{array}$ & $\begin{array}{c}1.580^{* * *} \\
(0.458) \\
6.371 * * * \\
(1.837) \\
-0.156 * * \\
(0.060)\end{array}$ & $\begin{array}{c}1.598 * * * \\
(0.443) \\
5.926 * * \\
(2.181) \\
-0.158 * * \\
(0.058)\end{array}$ \\
\hline $\begin{array}{l}\text { Servicios } \\
\text { Sociales }\end{array}$ & $\begin{array}{l}\text { Educación del padre } \\
\text { Log Gasto público per cápita } \\
\begin{array}{l}\text { Educación del padre x Log Gasto público per } \\
\text { cápita }\end{array}\end{array}$ & $\begin{array}{c}1.583 * * * \\
(0.356) \\
1.854 * \\
(1.062) \\
-0.165 * * * \\
(0.049)\end{array}$ & $\begin{array}{c}1.539 * * * \\
(0.352) \\
5.049 * * \\
(2.248) \\
-0.159 * * * \\
(0.048)\end{array}$ & $\begin{array}{c}1.541 * * * \\
(0.343) \\
4.054 \\
(2.748) \\
-0.160 * * * \\
(0.047)\end{array}$ \\
\hline GPSCH1 & $\begin{array}{l}\text { Educación del padre } \\
\text { Log Gasto público per cápita } \\
\begin{array}{l}\text { Educación del padre x Log Gasto público per } \\
\text { cápita }\end{array}\end{array}$ & $\begin{array}{c}1.459 * * * \\
(0.375) \\
1.540 * \\
(0.893) \\
-0.162 * * * \\
(0.057)\end{array}$ & $\begin{array}{c}1.394 * * * \\
(0.364) \\
5.143 * * * \\
(1.774) \\
-0.152 * * \\
(0.055)\end{array}$ & $\begin{array}{c}1.406 * * * \\
(0.352) \\
4.102 * \\
(2.013) \\
-0.154 * * * \\
(0.053)\end{array}$ \\
\hline GPSCH2 & $\begin{array}{l}\text { Educación del padre } \\
\text { Log Gasto público per cápita } \\
\begin{array}{l}\text { Educación del padre x Log Gasto público per } \\
\text { cápita }\end{array}\end{array}$ & $\begin{array}{c}1.495 * * * \\
(0.351) \\
1.641 * \\
(0.879) \\
-0.169 * * * \\
(0.053)\end{array}$ & $\begin{array}{c}1.436 * * * \\
(0.344) \\
3.701 * \\
(2.052) \\
-0.160 * * * \\
(0.052)\end{array}$ & $\begin{array}{c}1.444 * * * \\
(0.333) \\
4.701 * \\
(2.626) \\
-0.161 * * * \\
(0.050)\end{array}$ \\
\hline GPSCH3 & $\begin{array}{l}\text { Educación del padre } \\
\text { Log Gasto público per cápita } \\
\begin{array}{l}\text { Educación del padre x Log Gasto público per } \\
\text { cápita }\end{array}\end{array}$ & $\begin{array}{c}1.525^{* * *} \\
(0.328) \\
1.626^{*} \\
(0.851) \\
-0.174 * * * \\
(0.050)\end{array}$ & $\begin{array}{c}1.468 * * * \\
(0.323) \\
5.391 * * * \\
(1.724) \\
-0.165 * * * \\
(0.049)\end{array}$ & $\begin{array}{c}1.473 * * * \\
(0.311) \\
5.619 * * \\
(2.247) \\
-0.166 * * * \\
(0.047)\end{array}$ \\
\hline GPSCH4 & $\begin{array}{l}\text { Educación del padre } \\
\text { Log Gasto público per cápita } \\
\begin{array}{l}\text { Educación del padre x Log Gasto público per } \\
\text { cápita }\end{array}\end{array}$ & $\begin{array}{c}1.552 * * * \\
(0.306) \\
1.838 * * \\
(0.813) \\
-0.180 * * * \\
(0.047)\end{array}$ & $\begin{array}{c}1.492 * * * \\
(0.299) \\
6.257 * * * \\
(1.740) \\
-0.171 * * * \\
(0.046)\end{array}$ & $\begin{array}{c}1.498 * * * \\
(0.287) \\
5.977 * * * \\
(2.041) \\
-0.172 * * * \\
(0.044)\end{array}$ \\
\hline
\end{tabular}

Fuente: Elaboración propia sobre la base de varias fuentes de información. Ver la sección de datos para más detalles. Nota: $* * * p$-value $<0.01, * * p$-value $<0.05, *$-value $<0.1$. Entre paréntesis se reportan los errores estándares agrupados a nivel provincial. Ver la nota del Cuadro 3 con la descripción de la especificación de cada modelo. 
Por otra parte, es interesante notar que la magnitud de los términos de interacción que capturan el efecto del GPCpc en la EII y en el CPE, varían con la medida de inversión pública en capital humano de adolescentes y jóvenes que se utilice. En general, los efectos más elevados se obtienen al considerar las categorías de gasto más directamente relacionadas con la inversión pública en el capital humano de los jóvenes. Así, conforme a los coeficientes estimados para el modelo más completo, un incremento del 1\% en el GPSCH4 reduce la elasticidad intergeneracional del ingreso en 0.173 puntos porcentuales (p.p.) y la CPE en 0.172 p.p.

Una forma de evaluar la magnitud de los efectos estimados es comparándolos con los obtenidos por estudios similares que analicen el impacto del gasto público sobre alguna de estas medidas de inmovilidad intergeneracional. Pero, estos estudios son prácticamente inexistentes. No obstante, ML realizan estimaciones relativamente comparables, obteniendo un impacto negativo del gasto público total de los Estados Unidos sobre la EII de 0.128 , considerando un modelo con efectos fijos locales pero sin otros controles adicionales. Las estimaciones aquí obtenidas para el gasto público total, a partir de la especificación más simple similar a la de ML, indican que este efecto sería igual a 0.148. Otra forma de interpretar los términos de interacción estimados es evaluar la EII y el CPE en distintos niveles del gasto. En el Cuadro 5 se reportan las EII y los CPE estimados, considerando tres niveles del gasto (bajo, medio y alto) que surgen de dividir en terciles la distribución del logaritmo de las distintas medidas del gasto y evaluar la derivada del (log) ingreso o la educación del hijo con respecto al ingreso o la educación del padre en valor medio del gasto de cada tercil. Los resultados indican que un incremento del gasto público total de $\$ 1917$ (gasto promedio en el tercil más bajo) a \$2098 (el gasto promedio en el tercil más alto), es decir, en un 52\% reduce la EII estimada de 0.673 a 0.601 , esto es, en un $11 \%$ y el CPE de 0.403 a 0.338 , un $26 \%$. En el caso del GPSCH4, las estimaciones indican que un aumento de $55 \%$ en esta medida de gasto reduce la EII en un $11 \%$ y el CPE en un 18\%. Estos resultados sugieren que los efectos del GPCpc sobre ambas medidas de persistencia intergeneracional son modestos dado que se requiere un elevado incremento del gasto para conseguir una reducción económicamente significativa en los niveles de inmovilidad entre generaciones. 
Cuadro 5. EII y CPE estimados a partir del modelo de interacción para tres niveles de gasto

\begin{tabular}{|c|c|c|c|c|c|c|c|c|c|c|}
\hline \multirow{3}{*}{ Medida de gasto } & \multirow{3}{*}{ Modelo } & \multicolumn{3}{|c|}{ EII estimada } & \multicolumn{6}{|c|}{ Diferencias en el EII estimada según nivel de gasto } \\
\hline & & \multirow{2}{*}{ Gasto bajo } & \multirow{2}{*}{ Gasto medio } & \multirow{2}{*}{ Gasto alto } & \multicolumn{2}{|c|}{ Bajo - medio } & \multicolumn{2}{|c|}{ Medio - alto } & \multicolumn{2}{|c|}{ Bajo-alto } \\
\hline & & & & & En nivel & $E n \%$ & En nivel & $E n \%$ & En nivel & $E n \%$ \\
\hline \multirow[t]{3}{*}{ Total } & 1 & 0.671 & 0.658 & 0.609 & 0.013 & $2.0 \%$ & 0.048 & $7.3 \%$ & 0.062 & $10.1 \%$ \\
\hline & 2 & 0.671 & 0.658 & 0.609 & 0.013 & $2.0 \%$ & 0.048 & $7.3 \%$ & 0.062 & $10.1 \%$ \\
\hline & 3 & 0.673 & 0.657 & 0.601 & 0.016 & $2.3 \%$ & 0.057 & $8.6 \%$ & 0.072 & $12.0 \%$ \\
\hline \multirow[t]{3}{*}{ Servicios sociale } & 1 & 0.672 & 0.654 & 0.606 & 0.017 & $2.6 \%$ & 0.048 & $7.4 \%$ & 0.065 & $10.8 \%$ \\
\hline & 2 & 0.672 & 0.655 & 0.607 & 0.017 & $2.5 \%$ & 0.048 & $7.3 \%$ & 0.065 & $10.7 \%$ \\
\hline & 3 & 0.674 & 0.654 & 0.599 & 0.020 & $2.9 \%$ & 0.056 & $8.5 \%$ & 0.076 & $12.6 \%$ \\
\hline \multirow[t]{3}{*}{ GPSCH1 } & 1 & 0.670 & 0.657 & 0.610 & 0.013 & $2.0 \%$ & 0.046 & $7.0 \%$ & 0.059 & $9.7 \%$ \\
\hline & 2 & 0.670 & 0.657 & 0.611 & 0.013 & $2.0 \%$ & 0.046 & $7.0 \%$ & 0.060 & $9.7 \%$ \\
\hline & 3 & 0.672 & 0.657 & 0.604 & 0.015 & $2.3 \%$ & 0.053 & $8.0 \%$ & 0.068 & $11.3 \%$ \\
\hline \multirow[t]{3}{*}{ GPSCH2 } & 1 & 0.672 & 0.657 & 0.608 & 0.016 & $2.3 \%$ & 0.049 & $7.4 \%$ & 0.064 & $10.5 \%$ \\
\hline & 2 & 0.672 & 0.657 & 0.608 & 0.016 & $2.3 \%$ & 0.049 & $7.4 \%$ & 0.064 & $10.6 \%$ \\
\hline & 3 & 0.674 & 0.657 & 0.601 & 0.018 & $2.6 \%$ & 0.056 & $8.5 \%$ & 0.074 & $12.3 \%$ \\
\hline \multirow[t]{3}{*}{ GPSCH3 } & 1 & 0.671 & 0.656 & 0.607 & 0.015 & $2.3 \%$ & 0.048 & $7.3 \%$ & 0.064 & $10.5 \%$ \\
\hline & 2 & 0.672 & 0.656 & 0.607 & 0.016 & $2.3 \%$ & 0.049 & $7.4 \%$ & 0.064 & $10.6 \%$ \\
\hline & 3 & 0.673 & 0.656 & 0.600 & 0.018 & $2.7 \%$ & 0.056 & $8.5 \%$ & 0.074 & $12.3 \%$ \\
\hline \multirow[t]{3}{*}{ GPSCH4 } & 1 & 0.671 & 0.656 & 0.606 & 0.015 & $2.3 \%$ & 0.050 & $7.6 \%$ & 0.065 & $10.8 \%$ \\
\hline & 2 & 0.672 & 0.656 & 0.606 & 0.015 & $2.3 \%$ & 0.051 & $7.7 \%$ & 0.066 & $10.9 \%$ \\
\hline & 3 & 0.674 & 0.656 & 0.598 & 0.018 & $2.6 \%$ & 0.058 & $8.9 \%$ & 0.076 & $12.7 \%$ \\
\hline
\end{tabular}

\begin{tabular}{|c|c|c|c|c|c|c|c|c|c|c|}
\hline \multirow{3}{*}{ Medida de gasto } & \multirow{3}{*}{ Modelo } & \multicolumn{3}{|c|}{ CPE estimada } & \multicolumn{6}{|c|}{ Diferencias en el CPE estimada según nivel de gasto } \\
\hline & & \multirow{2}{*}{ Gasto bajo } & \multirow{2}{*}{ Gasto medio } & \multirow{2}{*}{ Gasto alto } & \multicolumn{2}{|c|}{ Bajo - medio } & \multicolumn{2}{|c|}{ Medio - alto } & \multicolumn{2}{|c|}{ Bajo-alto } \\
\hline & & & & & En nivel & $E n \%$ & En nivel & $E n \%$ & En nivel & $E n \%$ \\
\hline \multirow[t]{3}{*}{ Total } & 1 & 0.406 & 0.391 & 0.337 & 0.015 & $3.7 \%$ & 0.054 & $13.8 \%$ & 0.069 & $20.4 \%$ \\
\hline & 2 & 0.404 & 0.390 & 0.340 & 0.014 & $3.5 \%$ & 0.050 & $12.9 \%$ & 0.065 & $19.0 \%$ \\
\hline & 3 & 0.403 & 0.389 & 0.338 & 0.014 & $3.5 \%$ & 0.051 & $13.1 \%$ & 0.065 & $19.2 \%$ \\
\hline \multirow{3}{*}{$\begin{array}{l}\text { Servicios } \\
\text { sociales }\end{array}$} & 1 & 0.407 & 0.388 & 0.334 & 0.019 & $4.8 \%$ & 0.054 & $14.0 \%$ & 0.074 & $22.1 \%$ \\
\hline & 2 & 0.406 & 0.387 & 0.335 & 0.019 & $4.6 \%$ & 0.052 & $13.5 \%$ & 0.071 & $21.2 \%$ \\
\hline & 3 & 0.405 & 0.386 & 0.334 & 0.019 & $4.6 \%$ & 0.052 & $13.5 \%$ & 0.071 & $21.2 \%$ \\
\hline \multirow[t]{3}{*}{ GPSCH1 } & 1 & 0.407 & 0.392 & 0.338 & 0.015 & $3.8 \%$ & 0.053 & $13.6 \%$ & 0.069 & $20.3 \%$ \\
\hline & 2 & 0.405 & 0.390 & 0.340 & 0.014 & $3.6 \%$ & 0.050 & $12.8 \%$ & 0.065 & $19.0 \%$ \\
\hline & 3 & 0.404 & 0.389 & 0.339 & 0.015 & $3.6 \%$ & 0.050 & $12.9 \%$ & 0.065 & $19.2 \%$ \\
\hline \multirow[t]{3}{*}{ GPSCH2 } & 1 & 0.409 & 0.391 & 0.335 & 0.018 & $4.4 \%$ & 0.056 & $14.3 \%$ & 0.074 & $22.1 \%$ \\
\hline & 2 & 0.407 & 0.390 & 0.337 & 0.017 & $4.2 \%$ & 0.053 & $13.6 \%$ & 0.070 & $20.8 \%$ \\
\hline & 3 & 0.406 & 0.389 & 0.336 & 0.017 & $4.2 \%$ & 0.053 & $13.7 \%$ & 0.070 & $20.9 \%$ \\
\hline \multirow[t]{3}{*}{ GPSCH3 } & 1 & 0.408 & 0.390 & 0.333 & 0.018 & $4.5 \%$ & 0.057 & $14.5 \%$ & 0.075 & $22.5 \%$ \\
\hline & 2 & 0.406 & 0.389 & 0.335 & 0.017 & $4.3 \%$ & 0.054 & $13.9 \%$ & 0.071 & $21.3 \%$ \\
\hline & 3 & 0.405 & 0.388 & 0.334 & 0.017 & $4.3 \%$ & 0.054 & $13.9 \%$ & 0.071 & $21.3 \%$ \\
\hline \multirow[t]{3}{*}{ GPSCH4 } & 1 & 0.409 & 0.391 & 0.330 & 0.019 & $4.5 \%$ & 0.060 & $15.5 \%$ & 0.079 & $23.9 \%$ \\
\hline & 2 & 0.407 & 0.390 & 0.332 & 0.018 & $4.3 \%$ & 0.057 & $14.7 \%$ & 0.075 & $22.5 \%$ \\
\hline & 3 & 0.406 & 0.388 & 0.331 & 0.018 & $4.3 \%$ & 0.057 & $14.8 \%$ & 0.075 & $22.6 \%$ \\
\hline
\end{tabular}

Fuente: Elaboración propia sobre la base de estimaciones reportadas en los Cuadro 3 y 4

Nota: Ver la nota del Cuadro 3 con la descripción de la especificación de cada modelo.

En resumen, las estimaciones previas aportan evidencia a favor de la hipótesis de que la persistencia intergeneracional del ingreso y de la educación, medida a partir de la EII y el CPE, disminuyen con los incrementos del gasto público. Resulta importante recordar que los denominados Modelos 2 y 3, a partir de los cuales se derivan estas conclusiones, incluyen además de efectos fijos por provincia, por cohorte de nacimiento, dummies por año, controles por el sexo y la edad del hijo, la educación y edad del padre, un conjunto de controles por características provinciales como la tasa 
del crecimiento del PBG per cápita, los recursos tributarios recaudados por cada jurisdicción, la carga tributaria nacional por provincia, la tasa de pobreza por NBI, la desigualdad, la tasa de desocupación, la cantidad de población total, los años de escolaridad promedio de la PET, la tasa de matriculación en cada nivel educativo, la tasa de mortalidad total e infantil, entre otros. Además, cuando se considera el gasto público social así como subcategorías de éste, ambos modelos incorporan también la proporción del gasto total que se destina a todas las demás partidas, a fin de controlar por la correlación que existe entre ellas y aislar el efecto de las distintas medidas de GPSCH. Y el Modelo 3 incluye además interacciones entre las dummies provinciales y de año que permiten capturar tendencias específicas por provincia en el ingreso de los hijos.

La estrategia de identificación subyacente en las estimaciones previas, concretamente las obtenidas de la especificación más completa del modelo estimado que incluye efectos fijos por provincia, explota la variabilidad temporal del gasto público en cada provincia y no su variabilidad de corte transversal. Es decir, se asume que la incorporación de controles fijos por provincia elimina todos los factores locales constantes en el tiempo, correlacionados con la movilidad intergeneracional y el GPCpc como, por ejemplo, el clima social o político que podrían estar relacionadas tanto con el gasto público como con el ingreso de los hijos adultos. Además, se incluyen controles por características provinciales que varían por cohorte y pueden estar correlacionadas con el gasto público y con el ingreso paterno o de los hijos como, por ejemplo, la recaudación tributaria (medida a través de los recursos tributarios provinciales y la carga tributaria nacional por provincia vigentes para cada cohorte durante su adolescencia y juventud $)^{161}$. Si no se incluyeran estos controles por provincia, cualquier diferencia en las EII o en el CPE por niveles de gasto podría ser parcialmente endógena, entre otras razones, porque, dada la misma tasa de impuestos, los cambios en los ingresos de los residentes en una provincia se traduce en cambios en los ingresos públicos. También se incluyen controles por otras características provinciales que varían, no sólo localmente, sino también por cohorte como, por ejemplo, la tasa de pobreza por NBI, el nivel de

\footnotetext{
${ }^{161}$ Esta es una diferencia importante con la estrategia de identificación utilizada por Mayer y Lopoo (2008) que no incluyen en sus modelos estimados ningún control por factores locales que varían en el tiempo o por cohorte y que pueden estar correlacionados con las variables de interés, sesgando las estimaciones.
} 
desigualdad o la tasa de desocupación promedio existente en la provincia de residencia de cada hijo adulto cuando era adolescente o joven. Estos controles resultan relevantes porque algunas categorías de gasto público, particularmente del gasto público social, se ajustan a las necesidades existentes de manera que las provincias con más familias pobres tenderán a gastar más que las provincias con menos familias pobres. No obstante, aun cuando se incorporen efectos fijos por provincia y por año, interacciones entre estas dos variables, así como controles por distintas características de los hijos y padres y controles por características provinciales, las estimaciones del efecto del gasto público en la movilidad intergeneracional podrían estar sesgadas si persisten factores inobservables omitidos correlacionados con ambas variables. Por esto, a fin de analizar la robustez de estos resultados en la siguiente sección se presentan las estimaciones obtenidas con la técnica de diferencias en diferencias que permite, bajo determinados supuestos, eliminar los problemas de sesgo persistentes.

\subsection{Brechas de movilidad $y$ gasto público: resultados del método de diferencias en diferencias}

Conforme se afirmó en la sección 3, la técnica de diferencias en diferencias puede eliminar los problemas de sesgo persistentes en las estimaciones previas si el efecto de los factores inobservables correlacionados con las variables de interés es el mismo entre los hijos que experimentaron distintos niveles de gasto público per cápita en su provincia de residencia. Además este método permite también testear la hipótesis de la existencia de diferencias en las brechas de movilidad entre hijos de padres con alto y bajo nivel educativo, asociadas al nivel de gasto público per cápita ${ }^{162}$. Cuando se consideran las brechas en el CPE por nivel de gasto público, a fin de disminuir los problemas de multicolinealidad entre el término de interacción de interés y los años de educación del padre se considera como medida de trasfondo socioeconómico familiar el nivel educativo de la madre en lugar de la educación del padre ${ }^{163}$. Con este objetivo se

\footnotetext{
${ }^{162}$ El nivel educativo utilizado como umbral es el secundario completo, entonces, se considera que los padres con un nivel educativo mayor al secundario completo tienen un nivel educativo alto, ocurriendo lo contrario con aquellos padres con secundario completo o un nivel educativo menor.

${ }^{163}$ No obstante, la consideración del nivel educativo del padre como umbral para el cómputo de las brechas de movilidad no modifica sustancialmente las conclusiones obtenidas.
} 
estima el modelo (3) para tres grupos de hijos identificados según el nivel promedio de gasto público per cápita que experimentaron durante su juventud en sus provincias de residencia: bajo, medio y alto. En el Cuadro 6 (y Cuadro A1 del Anexo) se reportan estas estimaciones.

\section{Cuadro 6. Diferencias en diferencias del CPE por nivel educativo de la madre según nivel de gasto público}

\begin{tabular}{|c|c|c|c|c|c|c|c|c|c|}
\hline \multirow{3}{*}{$\begin{array}{c}\text { Medidas de gasto } \\
\text { público consolidado } \\
\text { per cápita }\end{array}$} & \multicolumn{9}{|c|}{ Difference in difference CPE } \\
\hline & \multicolumn{3}{|c|}{ Nivel de gasto bajo - medio } & \multicolumn{3}{|c|}{ Nivel de gasto medio - alto } & \multicolumn{3}{|c|}{ Nivel de gasto bajo-alto } \\
\hline & Mod. 1 & Mod. 2 & Mod. 3 & Mod. 1 & Mod. 2 & Mod. 3 & Mod. 1 & Mod. 2 & Mod. 3 \\
\hline Total & $\begin{array}{c}0.007 \\
(0.783)\end{array}$ & $\begin{array}{c}0.010 \\
(0.680)\end{array}$ & $\begin{array}{c}0.015 \\
(0.601)\end{array}$ & $\begin{array}{l}0.072 \quad * * * \\
(0.001)\end{array}$ & $\begin{array}{l}0.072 * * * \\
(0.001)\end{array}$ & $\begin{array}{l}0.066 * * \\
(0.010)\end{array}$ & $\begin{array}{l}0.079 * * * \\
(0.002)\end{array}$ & $\begin{array}{l}0.082 * * * \\
(0.001)\end{array}$ & $\begin{array}{l}0.081 \quad * * * * \\
(0.004)\end{array}$ \\
\hline Social total & $\begin{array}{l}-0.033 \\
(0.335)\end{array}$ & $\begin{array}{l}-0.033 \\
(0.321)\end{array}$ & $\begin{array}{l}-0.031 \\
(0.385)\end{array}$ & $\begin{array}{l}0.057 \quad * * \\
(0.023)\end{array}$ & $\begin{array}{l}0.056 * * \\
(0.043)\end{array}$ & $\begin{array}{l}0.053 * \\
(0.052)\end{array}$ & $\begin{array}{c}0.024 \\
(0.490)\end{array}$ & $\begin{array}{c}0.023 \\
(0.496)\end{array}$ & $\begin{array}{c}0.022 \\
(0.540)\end{array}$ \\
\hline GPSCHN1 & $\begin{array}{l}-0.006 \\
(0.855)\end{array}$ & $\begin{array}{l}-0.002 \\
(0.939)\end{array}$ & $\begin{array}{l}-0.003 \\
(0.924)\end{array}$ & $\begin{array}{l}0.059 * * \\
(0.028)\end{array}$ & $\begin{array}{l}0.058 * * \\
(0.043)\end{array}$ & $\begin{array}{l}0.054 * \\
(0.073)\end{array}$ & $\begin{array}{c}0.053 \\
(0.113)\end{array}$ & $\begin{array}{l}0.056 * \\
(0.081)\end{array}$ & $\begin{array}{c}0.051 \\
(0.155)\end{array}$ \\
\hline GPSCHN2 & $\begin{array}{c}0.024 \\
(0.382)\end{array}$ & $\begin{array}{c}0.026 \\
(0.311)\end{array}$ & $\begin{array}{c}0.022 \\
(0.434)\end{array}$ & $\begin{array}{c}0.040 \\
(0.224)\end{array}$ & $\begin{array}{l}0.040 \\
(0.267)\end{array}$ & $\begin{array}{c}0.039 \\
(0.293)\end{array}$ & $\begin{array}{l}0.064 \quad * * \\
(0.018)\end{array}$ & $\begin{array}{l}0.066 * * \\
(0.011)\end{array}$ & $\begin{array}{l}0.061 * * \\
(0.030)\end{array}$ \\
\hline GPSCHN3 & $\begin{array}{c}0.024 \\
(0.377)\end{array}$ & $\begin{array}{c}0.026 \\
(0.330)\end{array}$ & $\begin{array}{c}0.027 \\
(0.353)\end{array}$ & $\begin{array}{c}0.043 \\
(0.153)\end{array}$ & $\begin{array}{l}0.045 \\
(0.145)\end{array}$ & $\begin{array}{c}0.043 \\
(0.189)\end{array}$ & $\begin{array}{l}0.067 \quad * * \\
(0.013)\end{array}$ & $\mid \begin{array}{l}0.070 * * * \\
(0.008)\end{array}$ & $\begin{array}{l}0.070 * * \\
(0.015)\end{array}$ \\
\hline GPSCHN4 & $\begin{array}{l}-0.015 \\
(0.654)\end{array}$ & $\begin{array}{l}-0.011 \\
(0.743)\end{array}$ & $\begin{array}{l}-0.007 \\
(0.839)\end{array}$ & $\begin{array}{c}0.048 \\
(0.107)\end{array}$ & $\begin{array}{l}0.047 \\
(0.125)\end{array}$ & $\begin{array}{c}0.042 \\
(0.171)\end{array}$ & $\begin{array}{c}0.033 \\
(0.330)\end{array}$ & $\begin{array}{c}0.036 \\
(0.270)\end{array}$ & $\begin{array}{l}0.034 \\
(0.336)\end{array}$ \\
\hline
\end{tabular}

Fuente: Elaboración propia sobre la base de varias fuentes de información. Ver la sección de datos para más detalles.

Nota: $* * * p$-value $<0.01, * * p$-value $<0.05, * p$-value $<0.1$. Entre paréntesis, se reportan los $p$-values corregidos por la correlación intra-provincial entre las observaciones. Ver la nota del Cuadro 3 con la descripción de la especificación de cada modelo.

En general, se observan diferencias positivas en los CPE computados entre los hijos de padres con un nivel educativo superior al secundario completo y aquellos con madres que tienen una educación igual o inferior al secundario para aquellos que experimentaron niveles bajos o medios de gasto público (ver Cuadro A2 del Anexo) ${ }^{164}$. Además, esas diferencias resultan significativamente mayores para aquellos que residían, durante su adolescencia, en provincias con niveles de gasto público per cápita bajo en comparación con los que experimentaron niveles altos de GPCpc. La mayoría de las medidas consideradas de inversión pública en el capital humano de los jóvenes parecen tener un impacto significativo sobre las brechas de movilidad intergeneracional. Así, por ejemplo, de acuerdo con los resultados obtenidos del modelo más completo (reportados en el Cuadro A1), entre los hijos que experimentaron durante su adolescencia niveles de GPSCpc en salud, promoción social y educación relativamente bajos (GPSCH3), el CPE es mayor en 0.220 para quienes tienen una madre con un nivel

\footnotetext{
${ }^{164}$ Estas diferencias surgen del coeficiente $\delta_{2}$ en el modelo (3) que acompaña el término de interacción entre el logaritmo del ingreso predicho del padre y la dummy que indica si el nivel educativo del padre es bajo (igual o menor a secundario completo).
} 
educativo bajo en comparación con los hijos de madres con una educación mayor al secundario completo. En tanto que, entre aquellos que, durante su juventud, habitaban en provincias con altos niveles de gasto público en salud promoción social y educación, la brecha de movilidad intergeneracional asociada al nivel educativo de la madre asciende a 0.150 . Por tanto, la diferencia en los CPE por nivel educativo materno entre los hijos que experimentaron niveles de GPSCH3 bajo supera en 0.071 a la que se observa entre aquellos que experimentaron niveles altos de este gasto.

Cuando se considera como medida de persistencia socioeconómica intergeneracional la EII, sólo se obtienen efectos significativos del gasto público en las brechas de movilidad al comparar los hijos con niveles de gasto en el primer decil y aquellos en el quinto o en el último decil de la distribución del gasto público consolidado (ver Cuadro A3). Este resultado indica que se requiere un mayor incremento en el gasto público para reducir las brechas de movilidad intergeneracional por estrato socioeconómico cuando se utiliza como medida de bienestar o resultado económico de los hijos su ingreso en lugar de su educación.

\subsection{Las distintas categorías del gasto público y sus efectos en la movilidad intergeneracional}

Los resultados anteriores sugieren que el GPCpc, en particular el agregado de las categorías del gasto público social que parecen más estrechamente relacionadas con la inversión pública en capital humano de niños y adolescentes tienen un efecto significativo y positivo sobre la movilidad intergeneracional así como en las brechas de movilidad. No obstante, desde el punto de vista de la política pública, es relevante determinar también si algunas categorías particulares del gasto público social resultan más efectivas que otras para reducir los niveles de persistencia socioeconómica intergeneracional. La elevada correlación existente entre las distintas categorías de gasto dificulta la identificación del efecto individual de cada una. Por eso en todos los modelos estimados se incluye como variable de control la proporción del gasto destinada al conjunto de partidas no consideradas y su interacción con el ingreso y los años de educación del padre según corresponda. 
Los resultados de la estimación del modelo (2) para la MII se reportan en el Cuadro 7. Nuevamente, en todas las especificaciones el ingreso estimado del padre tiene un efecto positivo y estadísticamente significativo al 1\% sobre el ingreso del hijo y la mayoría de las categorías de gasto público consideradas presentan un efecto positivo en el ingreso de los hijos, particularmente en los Modelos 2 y 3 que incluyen controles por un conjunto de características provinciales que están correlacionadas positivamente con algunas categorías del gasto público pero negativamente con el ingreso de los hijos. Sin embargo, este efecto positivo del gasto público en el ingreso de los hijos no es significativo en el caso de la educación básica. Esta falta de significancia estadística podría deberse en parte al hecho de que esta partida del gasto no beneficia directamente a la mayoría de los jóvenes de 18 a 24 años ${ }^{165}$ sino a los niños y adolescentes.

Los términos de interacción entre el GPCpc y el ingreso del padre tienen el signo esperado, siendo negativos para casi todas las categorías de gasto público social y estadísticamente significativos en las tres especificaciones, salvo en el caso del gasto en vivienda y urbanismo. Esto podría estar relacionado con la incidencia distributiva de algunas partidas de este gasto (como las asociadas con planes asignados con contrapartida de cuotas) que no tienen, a diferencia de las restantes, una claro sesgo propobre. En general, este sector es quizás el que presenta mayor variabilidad en los resultados de los estudios incidencia distributiva (Gasparini et al., 2000). Algunas de estas investigaciones, como la desarrollada por Paqueo y Lee (2000), encuentran un incidencia uniforme y no pro-pobre del gasto en vivienda.

Asimismo, cuando se considera el GPCpc en agua y alcantarillado, los términos de interacción negativos entre esta categoría de gasto y el ingreso paterno predicho sólo resultan estadísticamente significativos en las dos especificaciones más completa del modelo. En principio, estos resultados indican que a mayores niveles de gasto público (en las principales categorías del gasto público social) experimentados en la juventud, menor es la persistencia intergeneracional de ingresos medida por la EII.

Las estimaciones correspondientes al modelo intergeneracional educativo se muestran en el Cuadro 8.

${ }^{165}$ A excepción de aquellos que cursen con sobreedad los estudios primarios o secundarios. 


\section{Cuadro 7. Efecto de las categorías del gasto público social consolidado per cápita sobre la elasticidad intergeneracional del ingreso}

\begin{tabular}{|c|c|c|c|c|}
\hline \begin{tabular}{|l} 
Categorías \\
GPC \\
\end{tabular} & Variables de interés & Mod. 1 & Mod. 2 & Mod. 3 \\
\hline Salud & $\begin{array}{l}\text { Log del Ingreso del padre } \\
\text { Log Gasto público per cápita } \\
\text { Log del Ingreso del padre x Log Gasto público per } \\
\text { cápita }\end{array}$ & $\begin{array}{c}1.350 * * * \\
(0.201) \\
0.157 \\
(0.164) \\
-0.121 * * * \\
(0.034)\end{array}$ & $\begin{array}{c}1.388^{* * *} \\
(0.218) \\
1.008 * * \\
(0.398) \\
-0.134 * * \\
(0.049)\end{array}$ & $\begin{array}{c}1.513 * * * \\
(0.191) \\
1.267 * * * \\
(0.330) \\
-0.155^{* * *} \\
(0.045)\end{array}$ \\
\hline $\begin{array}{l}\text { Atención } \\
\text { médica }\end{array}$ & $\begin{array}{l}\text { Log del Ingreso del padre } \\
\text { Log Gasto público per cápita } \\
\text { Log del Ingreso del padre x Log Gasto público per } \\
\text { cápita }\end{array}$ & $\begin{array}{c}1.119 * * * \\
(0.143) \\
0.269 \\
(0.169) \\
-0.097 * * * \\
(0.029) \\
\end{array}$ & $\begin{array}{c}1.229 * * * \\
(0.196) \\
0.967 * * * \\
(0.343) \\
-0.135 * * \\
(0.059) \\
\end{array}$ & $\begin{array}{c}1.308 * * * \\
(0.181) \\
0.817 * * * \\
(0.273) \\
-0.154 * * \\
(0.056) \\
\end{array}$ \\
\hline Obras sociales & $\begin{array}{l}\text { Log del Ingreso del padre } \\
\text { Log Gasto público per cápita } \\
\text { Log del Ingreso del padre x Log Gasto público per } \\
\text { cápita }\end{array}$ & $\begin{array}{c}1.205^{* * *} \\
(0.142) \\
0.170 \\
(0.130) \\
-0.112 * * * \\
(0.027) \\
\end{array}$ & $\begin{array}{c}1.265^{* * *} \\
(0.155) \\
1.185^{* * *} \\
(0.388) \\
-0.146^{* * *} \\
(0.041) \\
\end{array}$ & $\begin{array}{c}1.385 * * * \\
(0.133) \\
1.672 * * * \\
(0.377) \\
-0.170 * * * \\
(0.039) \\
\end{array}$ \\
\hline $\begin{array}{l}\text { Promoción y } \\
\text { Asistencia } \\
\text { Social }\end{array}$ & $\begin{array}{l}\text { Log del Ingreso del padre } \\
\text { Log Gasto público per cápita } \\
\text { Log del Ingreso del padre x Log Gasto público per } \\
\text { cápita }\end{array}$ & $\begin{array}{c}1.077 * * * \\
(0.159) \\
0.286 \\
(0.194) \\
-0.099 * * \\
(0.036)\end{array}$ & $\begin{array}{c}1.178^{* * *} \\
(0.162) \\
1.095 * * * \\
(0.318) \\
-0.155^{* * *} \\
(0.040)\end{array}$ & $\begin{array}{c}1.281 * * * \\
(0.144) \\
0.819 * * \\
(0.316) \\
-0.187 * * * \\
(0.035)\end{array}$ \\
\hline $\begin{array}{l}\text { Promoción y } \\
\text { asistencia } \\
\text { social pública }\end{array}$ & $\begin{array}{l}\text { Log del Ingreso del padre } \\
\text { Log Gasto público per cápita } \\
\text { Log del Ingreso del padre x Log Gasto público per } \\
\text { cápita }\end{array}$ & $\begin{array}{c}1.001 * * * \\
(0.122) \\
0.391 * * \\
(0.179) \\
-0.098 * * * \\
(0.033) \\
\end{array}$ & $\begin{array}{c}1.108 * * * \\
(0.107) \\
1.179 * * * \\
(0.219) \\
-0.155^{* * *} \\
(0.034) \\
\end{array}$ & $\begin{array}{c}1.189 * * * \\
(0.103) \\
1.175 * * * \\
(0.187) \\
-0.188 * * * \\
(0.032) \\
\end{array}$ \\
\hline $\begin{array}{l}\text { Educación y } \\
\text { Cultura }\end{array}$ & $\begin{array}{l}\text { Log del Ingreso del padre } \\
\text { Log Gasto público per cápita } \\
\text { Log del Ingreso del padre x Log Gasto público per } \\
\text { cápita }\end{array}$ & $\begin{array}{c}1.555^{* * *} \\
(0.256) \\
0.396^{*} \\
(0.221) \\
-0.158^{* * *} \\
(0.044)\end{array}$ & $\begin{array}{c}1.420 * * * \\
(0.259) \\
0.389 \\
(0.431) \\
-0.155^{* * *} \\
(0.040)\end{array}$ & $\begin{array}{c}1.426^{* * *} \\
(0.259) \\
0.659 * \\
(0.374) \\
-0.173 * * * \\
(0.040)\end{array}$ \\
\hline $\begin{array}{l}\text { Educación } \\
\text { básica }\end{array}$ & $\begin{array}{l}\text { Log del Ingreso del padre } \\
\text { Log Gasto público per cápita } \\
\text { Log del Ingreso del padre x Log Gasto público per } \\
\text { cápita }\end{array}$ & $\begin{array}{c}1.164 * * * \\
(0.298) \\
0.128 \\
(0.277) \\
-0.097 * \\
(0.056)\end{array}$ & $\begin{array}{c}1.255^{* * *} \\
(0.285) \\
0.069 \\
(0.533) \\
-0.148^{* *} \\
(0.053)\end{array}$ & $\begin{array}{c}1.318 * * * \\
(0.277) \\
0.363 \\
(0.405) \\
-0.169 * * * \\
(0.051)\end{array}$ \\
\hline $\begin{array}{l}\text { Educación } \\
\text { superior y } \\
\text { universitaria }\end{array}$ & $\begin{array}{l}\text { Log del Ingreso del padre } \\
\text { Log Gasto público per cápita } \\
\text { Log del Ingreso del padre x Log Gasto público per } \\
\text { cápita }\end{array}$ & $\begin{array}{c}1.024 * * * \\
(0.089) \\
0.130 \\
(0.118) \\
-0.091 * * * \\
(0.021)\end{array}$ & $\begin{array}{c}1.183 * * * \\
(0.136) \\
1.040 * * * \\
(0.349) \\
-0.166^{* * *} \\
(0.046) \\
\end{array}$ & $\begin{array}{c}1.282 * * * \\
(0.114) \\
1.397 * * * \\
(0.269) \\
-0.196 * * * \\
(0.041)\end{array}$ \\
\hline $\begin{array}{l}\text { Vivienda y } \\
\text { Urbanismo }\end{array}$ & $\begin{array}{l}\text { Log del Ingreso del padre } \\
\text { Log Gasto público per cápita } \\
\text { Log del Ingreso del padre x Log Gasto público per } \\
\text { cápita }\end{array}$ & $\begin{array}{c}0.602 * * * \\
(0.064) \\
-0.155 \\
(0.112) \\
0.012 \\
(0.020)\end{array}$ & $\begin{array}{c}0.610 * * * \\
(0.078) \\
-0.346 \\
(0.206) \\
0.010 \\
(0.034)\end{array}$ & $\begin{array}{c}0.666^{* * *} \\
(0.077) \\
-0.357^{* *} \\
(0.165) \\
-0.015 \\
(0.036)\end{array}$ \\
\hline $\begin{array}{l}\text { Agua y } \\
\text { alcantarillado }\end{array}$ & $\begin{array}{l}\text { Log del Ingreso del padre } \\
\text { Log Gasto público per cápita } \\
\text { Log del Ingreso del padre x Log Gasto público per } \\
\text { cápita }\end{array}$ & $\begin{array}{c}0.672 * * * \\
(0.046) \\
0.007 \\
(0.089) \\
-0.012 \\
(0.016) \\
\end{array}$ & $\begin{array}{c}0.738 * * * \\
(0.053) \\
0.352 * * \\
(0.145) \\
-0.058 * * \\
(0.027) \\
\end{array}$ & $\begin{array}{c}0.740 * * * \\
(0.051) \\
0.381 * * \\
(0.161) \\
-0.062 * * \\
(0.029)\end{array}$ \\
\hline
\end{tabular}

Fuente: Elaboración propia sobre la base de varias fuentes de información. Ver la sección de datos para más detalles. Nota: *** p-value $<0.01, * *$ p-value $<0.05$, * p-value $<0.1$. Entre paréntesis se reportan los errores estándares agrupados a nivel provincial. Ver la nota del Cuadro 3 con la descripción de la especificación de cada modelo. 


\section{Cuadro 8. Efecto de las categorías del gasto público social consolidado per cápita sobre el coeficiente de persistencia educativa intergeneracional}

\begin{tabular}{|c|c|c|c|c|}
\hline $\begin{array}{l}\text { Categorías } \\
\text { GPC }\end{array}$ & Variables de interés & Mod. 1 & Mod. 2 & Mod. 3 \\
\hline Salud & $\begin{array}{l}\text { Educación del padre } \\
\text { Log Gasto público per cápita } \\
\begin{array}{l}\text { Educación del padre x Log Gasto público per } \\
\text { cápita }\end{array}\end{array}$ & $\begin{array}{c}1.233 * * * \\
(0.191) \\
1.397 * * \\
(0.650) \\
-0.147 * * * \\
(0.032) \\
\end{array}$ & $\begin{array}{c}1.124 * * * \\
(0.168) \\
5.559 * * * \\
(1.631) \\
-0.115 * * * \\
(0.031) \\
\end{array}$ & $\begin{array}{c}1.136 * * * \\
(0.167) \\
5.403 * * * \\
(1.883) \\
-0.120^{* * *} \\
(0.032) \\
\end{array}$ \\
\hline $\begin{array}{l}\text { Atención } \\
\text { médica }\end{array}$ & $\begin{array}{l}\text { Educación del padre } \\
\text { Log Gasto público per cápita } \\
\begin{array}{l}\text { Educación del padre x Log Gasto público per } \\
\text { cápita }\end{array}\end{array}$ & $\begin{array}{c}0.917 * * * \\
(0.118) \\
0.668 \\
(0.452) \\
-0.110 * * * \\
(0.024) \\
\end{array}$ & $\begin{array}{c}0.778 * * * \\
(0.155) \\
2.925 \\
(1.730) \\
-0.066 \\
(0.043) \\
\end{array}$ & $\begin{array}{c}0.808 * * * \\
(0.159) \\
1.662 \\
(1.764) \\
-0.077 * \\
(0.044) \\
\end{array}$ \\
\hline Obras sociales & $\begin{array}{l}\text { Educación del padre } \\
\text { Log Gasto público per cápita } \\
\begin{array}{l}\text { Educación del padre x Log Gasto público per } \\
\text { cápita }\end{array}\end{array}$ & $\begin{array}{c}1.176^{* * *} \\
(0.188) \\
1.949 * * * \\
(0.587) \\
-0.160 * * * \\
(0.038) \\
\end{array}$ & $\begin{array}{c}1.088 * * * \\
(0.172) \\
4.684 * * * \\
(1.554) \\
-0.126 * * * \\
(0.036) \\
\end{array}$ & $\begin{array}{c}1.096 * * * \\
(0.167) \\
4.921 * * \\
(1.991) \\
-0.130 * * * \\
(0.035) \\
\end{array}$ \\
\hline $\begin{array}{l}\text { Promoción y } \\
\text { Asistencia } \\
\text { Social }\end{array}$ & $\begin{array}{l}\text { Educación del padre } \\
\text { Log Gasto público per cápita } \\
\begin{array}{l}\text { Educación del padre x Log Gasto público per } \\
\text { cápita }\end{array}\end{array}$ & $\begin{array}{c}0.924 * * * \\
(0.219) \\
0.486 \\
(0.667) \\
-0.124 * * \\
(0.051) \\
\end{array}$ & $\begin{array}{c}0.980 * * * \\
(0.186) \\
2.898 * * \\
(1.261) \\
-0.169 * * * \\
(0.056) \\
\end{array}$ & $\begin{array}{c}0.988 * * * \\
(0.181) \\
3.951 * * \\
(1.495) \\
-0.171 * * * \\
(0.054) \\
\end{array}$ \\
\hline $\begin{array}{l}\text { Promoción y } \\
\text { asistencia } \\
\text { social pública }\end{array}$ & $\begin{array}{l}\text { Educación del padre } \\
\text { Log Gasto público per cápita } \\
\begin{array}{l}\text { Educación del padre x Log Gasto público per } \\
\text { cápita }\end{array}\end{array}$ & $\begin{array}{c}0.673 * * * \\
(0.129) \\
0.523 \\
(0.551) \\
-0.080 * * \\
(0.038)\end{array}$ & $\begin{array}{c}0.880 * * * \\
(0.138) \\
2.803 * * * \\
(0.901) \\
-0.186 * * * \\
(0.052)\end{array}$ & $\begin{array}{c}0.886 * * * \\
(0.134) \\
3.872 * * * \\
(0.953) \\
-0.187 * * * \\
(0.050)\end{array}$ \\
\hline $\begin{array}{l}\text { Educación y } \\
\text { Cultura }\end{array}$ & $\begin{array}{l}\text { Educación del padre } \\
\text { Log Gasto público per cápita } \\
\begin{array}{l}\text { Educación del padre x Log Gasto público per } \\
\text { cápita }\end{array}\end{array}$ & $\begin{array}{c}1.301 * * * \\
(0.445) \\
1.782 \\
(1.085) \\
-0.160 * \\
(0.078)\end{array}$ & $\begin{array}{c}1.032 * * * \\
(0.272) \\
5.368 * * \\
(2.240) \\
-0.149 * * * \\
(0.050)\end{array}$ & $\begin{array}{c}1.062 * * * \\
(0.277) \\
4.522 * \\
(2.468) \\
-0.153 * * * \\
(0.049)\end{array}$ \\
\hline $\begin{array}{l}\text { Educación } \\
\text { básica }\end{array}$ & $\begin{array}{l}\text { Educación del padre } \\
\text { Log Gasto público per cápita } \\
\begin{array}{l}\text { Educación del padre x Log Gasto público per } \\
\text { cápita }\end{array}\end{array}$ & $\begin{array}{c}0.747 * * * \\
(0.234) \\
0.886 \\
(0.581) \\
-0.068 \\
(0.046)\end{array}$ & $\begin{array}{c}0.824 * * * \\
(0.187) \\
3.486 \\
(2.355) \\
-0.129 * * * \\
(0.041)\end{array}$ & $\begin{array}{c}0.855^{* * *} \\
(0.190) \\
2.644 \\
(2.629) \\
-0.135 * * * \\
(0.042)\end{array}$ \\
\hline $\begin{array}{l}\text { Educación } \\
\text { superior y } \\
\text { universitaria }\end{array}$ & $\begin{array}{l}\text { Educación del padre } \\
\text { Log Gasto público per cápita } \\
\begin{array}{l}\text { Educación del padre x Log Gasto público per } \\
\text { cápita }\end{array}\end{array}$ & $\begin{array}{c}0.922 * * * \\
(0.152) \\
1.531 * * \\
(0.646) \\
-0.130 * * * \\
(0.037) \\
\end{array}$ & $\begin{array}{c}1.003 * * * \\
(0.135) \\
2.246 * * \\
(0.912) \\
-0.174 * * * \\
(0.042) \\
\end{array}$ & $\begin{array}{c}1.006 * * * \\
(0.132) \\
3.475 * * \\
(1.398) \\
-0.177 * * * \\
(0.041)\end{array}$ \\
\hline $\begin{array}{l}\text { Vivienda y } \\
\text { Urbanis mo }\end{array}$ & $\begin{array}{l}\text { Educación del padre } \\
\text { Log Gasto público per cápita } \\
\begin{array}{l}\text { Educación del padre x Log Gasto público per } \\
\text { cápita }\end{array}\end{array}$ & $\begin{array}{c}0.390 * * * \\
(0.063) \\
-0.139 \\
(0.300) \\
-0.003 \\
(0.020) \\
\end{array}$ & $\begin{array}{c}0.571 * * * \\
(0.126) \\
-0.857 \\
(0.543) \\
-0.081 \\
(0.055) \\
\end{array}$ & $\begin{array}{c}0.584 * * * \\
(0.128) \\
-1.418 * * \\
(0.532) \\
-0.086 \\
(0.056) \\
\end{array}$ \\
\hline $\begin{array}{l}\text { Agua y } \\
\text { alcantarillado }\end{array}$ & $\begin{array}{l}\text { Educación del padre } \\
\text { Log Gasto público per cápita } \\
\begin{array}{l}\text { Educación del padre x Log Gasto público per } \\
\text { cápita }\end{array}\end{array}$ & $\begin{array}{c}0.361 * * * \\
(0.051) \\
0.173 \\
(0.133) \\
0.008 \\
(0.018)\end{array}$ & $\begin{array}{c}0.421 * * * \\
(0.063) \\
0.802 * \\
(0.444) \\
-0.037 \\
(0.038)\end{array}$ & $\begin{array}{c}0.425^{* * *} \\
(0.063) \\
0.635 \\
(0.499) \\
-0.040 \\
(0.038)\end{array}$ \\
\hline
\end{tabular}

Fuente: Elaboración propia sobre la base de varias fuentes de información. Ver la sección de datos para más detalles.

Nota: *** p-value $<0.01, * * p$-value $<0.05, * p$-value $<0.1$. Entre paréntesis se reportan los errores estándares agrupados a nivel provincial. Ver la nota del Cuadro 3 con la descripción de la especificación de cada modelo. 
Se observa que la educación del padre tiene, en casi la totalidad de los modelos estimados, un efecto positivo y estadísticamente significativo (a un nivel de significancia del 1\%) sobre los años de educación obtenido por sus hijos. Los coeficientes del GPCpc presentan también signos positivos en la mayoría de las categorías consideradas. Los términos de interacción entre el GPCpc y la educación del padre, por su parte, tienen en casi todas las categorías, el signo negativo esperado, siendo además estadísticamente significativos, en los tres modelos estimados, en el caso del GPCpc en salud (su subcategoría, obras sociales), en promoción y asistencia social, en educación total, media y superior.

Asimismo, la magnitud del efecto de las categorías del GPSCpc sobre la EII y el CPE varía según la partida de gasto social considerada. Cuando se consideran los términos de interacción estimados para el modelo intergeneracional más completo (Mod. 3) se aprecia que el gasto en promoción y asistencia social así como el gasto en educación superior son las dos categorías de GPSCpc con el mayor impacto sobre la EII y en el CPE. El efecto comparativamente más alto del gasto en educación superior en ambas medidas de persistencia intergeneracional probablemente esté relacionado con el tramo de edad de los hijos en la que se mide la inversión pública en capital humano: 18 a 24 años, siendo este grupo etario el principal beneficiario directo de esta partida del gasto educativo. En resumen, los resultados de los Cuadros 6 y 7 sugieren que determinadas categorías del gasto público, especialmente las que tienen un impacto directo sobre el capital humano, son más efectivas que otras para aumentar la movilidad intergeneracional.

Por otra parte, resulta de interés también examinar si alguna de las categorías del gasto público que no se destina a servicios sociales tiene un efecto significativo sobre las medidas de persistencia intergeneracional analizadas. En particular, el gasto público en seguridad y defensa podría tener un impacto sobre la movilidad entre generaciones que puede ser positivo o negativo dependiendo de cómo esta categoría de gasto afecta otras variables como, por ejemplo, la desigualdad y el crecimiento que tienen, a su vez, un impacto en la MI. Existen distintos argumentos que explican las relaciones potenciales entre gasto militar y bienestar económico. Algunos fundamentan la existencia de una relación positiva entre este tipo de gasto y el crecimiento económico así como una negativa entre aquel y la desigualdad en la distribución del ingreso, 
señalando que un incremento en el gasto militar aumenta la demanda agregada y el empleo en la economía (Benoit, 1978). Como esta expansión en la economía beneficia relativamente más a los pobres mejora la distribución del ingreso. Knight et al. (1996) en su estudio para 44 países, ofrece amplia evidencia de que el gasto en defensa tiene un impacto positivo sobre el crecimiento. No obstante, otra corriente de argumentos postula la ausencia de cualquier relación causal entre gasto militar y crecimiento económico (Biswas y Ram, 1986; Payne y Ross, 1992; Kim, 1996; Dakurah et al., 2001. En cambio, según otros autores un mayor gasto en defensa disminuye los fondos destinados a partidas sociales como educación, salud y promoción social, retardando el crecimiento económico al reducir la inversión en capital humano. Este argumento, denominado del "crowding-out" del gasto militar (o "guns-and-butter argument"), fue articulado originalmente por Russet (1969) en base a evidencia empírica para Estados Unidos, Francia y el Reino Unido. No obstante, en un estudio seminal Yildrim y Sezgin (2002) mostraron que existe un trade-off negativo entre defensa y salud pero uno positivo entre defensa y educación en Turquía. Según Ali (2011), los resultados de Russet (1969) podrían reflejar la dinámica subyacente de las economías desarrolladas, mientras que los hallazgos de Yildirim y Sezgin (2002) representan mejor la situación de un país en desarrollo con un conjunto de instituciones diferentes. En definitiva, no existen resultados concluyentes en la literatura sobre las relaciones entre gasto en defensa y bienestar. Por tanto, es difícil adelantar alguna hipótesis acerca del efecto potencial del gasto en defensa o seguridad interior sobre la movilidad intergeneracional.

En el Cuadro 9 se presentan las estimaciones del modelo de interacción para el gasto en seguridad y defensa total así como para el gasto en seguridad interior. Los resultados obtenidos sugieren que ambas partidas del gasto público tienen un efecto positivo y significativo tanto sobre el ingreso como sobre la educación de los hijos. Además, el signo negativo y estadísticamente significativo del término de interacción bajo análisis sugiere que un incremento en el gasto en defensa o en seguridad reducen las medidas de persistencia intergeneracional consideradas (la EII y el CPE). No obstante, los resultados deberían ser interpretados con precaución debido a la elevada correlación positiva existente entre el gasto en seguridad y el gasto en servicios sociales $(r=0.95)$ que dificultan aislar el efecto individual de cada tipo de gasto. 
Cuadro 9. Efecto del gasto público consolidado en defensa y seguridad sobre las medidas de persistencia intergeneracional

\begin{tabular}{|c|c|c|c|c|}
\hline $\begin{array}{l}\text { Medidas } \\
\text { GPCpc }\end{array}$ & Variables de interés & Mod. 1 & Mod. 2 & Mod. 3 \\
\hline \multicolumn{5}{|c|}{ Efectos en el coeficiente de persitencia educativa } \\
\hline \multirow[t]{5}{*}{$\begin{array}{l}\text { Seguridad y } \\
\text { defensa }\end{array}$} & Educación del padre & $\begin{array}{c}1.304 * * * \\
(0.255)\end{array}$ & $\begin{array}{c}1.277 * * * \\
(0.252)\end{array}$ & $\begin{array}{c}1.282 * * * \\
(0.242)\end{array}$ \\
\hline & Log Gasto público per cápita & $2.268^{* *}$ & $4.929 * * *$ & $4.730 * *$ \\
\hline & & $(0.887)$ & $(1.646)$ & $(1.946)$ \\
\hline & Educación del padre $\times$ Log Gasto público per & $-0.183 * * *$ & $-0.178 * * *$ & $-0.179 * * *$ \\
\hline & cápita & $(0.050)$ & $(0.050)$ & $(0.048)$ \\
\hline \multirow{6}{*}{$\begin{array}{l}\text { Seguridad } \\
\text { interior }\end{array}$} & Educación del padre & $0.930 * * *$ & $0.902 * * *$ & $0.905 * * *$ \\
\hline & & $(0.138)$ & $(0.138)$ & $(0.131)$ \\
\hline & Log Gasto público per cápita & $1.324 *$ & $5.840 * * *$ & $5.714 * *$ \\
\hline & & $(0.644)$ & $(1.720)$ & $(2.044)$ \\
\hline & Educación del padre $\times$ Log Gasto público per & $-0.122 * * *$ & $-0.116^{* * *}$ & $-0.117 * * *$ \\
\hline & cápita & $(0.030)$ & $(0.030)$ & $(0.029)$ \\
\hline \multicolumn{5}{|c|}{ Efectos en la elasticidad intergeneracional del ingreso } \\
\hline \multirow[t]{4}{*}{$\begin{array}{l}\text { Seguridad y } \\
\text { defensa }\end{array}$} & Log del ingreso del padre & $\begin{array}{c}1.468 * * * \\
(0.230)\end{array}$ & $\begin{array}{c}1.481 * * * \\
(0.234)\end{array}$ & $\begin{array}{c}1.631^{* * *} \\
(0.200)\end{array}$ \\
\hline & Log del Gasto público per cápita & 0.262 & $1.367 * *$ & $1.570 * * *$ \\
\hline & & $(0.218)$ & $(0.540)$ & $(0.533)$ \\
\hline & $\begin{array}{l}\text { Log del ingreso del padre } \times \text { Log del Gasto } \\
\text { público per cápita }\end{array}$ & $\begin{array}{c}-0.162 * * * \\
(0.044)\end{array}$ & $\begin{array}{c}-0.165^{* * *} \\
(0.045)\end{array}$ & $\begin{array}{c}-0.195 * * * \\
(0039)\end{array}$ \\
\hline \multirow{6}{*}{$\begin{array}{l}\text { Seguridad } \\
\text { interior }\end{array}$} & Log del ingreso del padre & $1.044 * * *$ & $1.054 * * *$ & $1.149 * * *$ \\
\hline & & (0.122) & (0.122) & (0.109) \\
\hline & Log del Gasto público per cápita & -0.058 & 0.716 & $0.963 * *$ \\
\hline & & $(0.124)$ & $(0.482)$ & $(0.424)$ \\
\hline & Log del ingreso del padre $\times$ Log del Gasto & $-0.088 * * *$ & $-0.090 * * *$ & $-0.111 * * *$ \\
\hline & público per cápita & $(0.025)$ & $(0.025)$ & $(0.022)$ \\
\hline
\end{tabular}

Fuente: Elaboración propia sobre la base de varias fuentes de información. Ver la sección de datos para más detalles.

Nota: $* * * p$-value $<0.01, * * p$-value $<0.05, * p$-value $<0.1$. Entre paréntesis se reportan los errores estándares agrupados a nivel provincial. Ver la nota del Cuadro 3 con la descripción de la especificación de cada modelo.

\subsection{Gasto público y movilidad intergeneracional entre estratos de ingresos}

Los resultados analizados en las secciones previas sugieren que el gasto público redujo los niveles promedio de persistencia socioeconómica intergeneracional. Pero no permiten saber si esto se debe a un incremento en la movilidad ascendente o en la movilidad descendente de los hijos con relación a la situación original de sus padres. A fin de examinar este punto se considera como medida de movilidad ascendente ${ }^{166}$ la probabilidad de que el percentil del hijo en la distribución de ingresos de su cohorte supere el percentil en el que puede clasificarse a su padre en función de su ingreso

${ }^{166}$ Esta medida fue introducida por Bhattacharya y Mazumder (2011). 
predicho $^{167}$. Formalmente, sea $Y_{h i}$ y $Y_{p i}$ el ingreso del i-ésimo hijo y padre con funciones de distribución acumulada marginales $F_{h i}$ y $F_{p i}$ respectivamente, la movilidad ascendente se define como:

$$
v_{i}=\operatorname{Prob}\left\lfloor F_{h i}-F_{p i}>\tau\right\rfloor(4)
$$

Esta medida de movilidad, a diferencia de las probabilidades de transición normalmente consideradas, tiene la ventaja de permitir considerar movimientos pequeños de los hijos en su posición relativa a la de sus padres, que son ignorados por las probabilidades de transición. En efecto, el valor de $\tau$ controla la magnitud de los movimientos en la posición relativa, de manera que a menor $\tau$, se considerarán movimientos más pequeños.

A fin de examinar el efecto del gasto público en esta medida de movilidad ascendente se estima el siguiente modelo probit:

$$
\begin{aligned}
\operatorname{Prob}\left[v_{i}=1\right] & =\operatorname{Prob}\left[F_{h i}-F_{p i}>\tau \mid x\right] \\
& =\Phi\left[\alpha+\delta_{1} G_{c j}+\gamma A_{i c j}+\theta X_{c j}+\varphi D_{j}+\pi D_{t}+\lambda D_{c}+\vartheta\left(D_{t} \times D_{j}\right)+\varepsilon_{i c j t}\right]
\end{aligned}
$$

donde $\Phi[\cdot]$ es la función de distribución normal acumulada estándar, c indexa la cohorte de nacimiento, $\mathrm{j}$ la provincia de residencia, y $\mathrm{t}$ el año, $G_{c j}$ es el logaritmo del gasto público consolidado per cápita; $A_{i c j}$ son controles individuales que incluyen el sexo del hijo, un polinomio de grado cuarto en su edad, un polinomio cuadrático en la edad del padre; $X_{c j}$ es un vector de variables de control a nivel provincial que varían por cohorte previamente descripto en la sección 3.

Los resultados de la estimación del modelo probit para $\tau=0$ se muestran en el Cuadro 10. Conforme se esperaba, todas las medidas consideradas de gasto público tienen efectos positivos y significativos sobre la probabilidad de movilidad de los hijos de ubicarse en un percentil superior al de su padre, para las dos especificaciones más completas del modelo (5). Según los efectos marginales computados a partir del modelo

\footnotetext{
${ }^{167}$ Ver sección 3 para más detalles metodológicos relacionados con esta estimación. Cabe aclarar que para clasificar a los hijos en los percentiles de la distribución del ingreso de su generación también se consideró su ingreso predicho utilizando la misma ecuación de ingreso empleada para predecir el ingreso de los padres. De otra forma, si se utilizara el ingreso observado del hijo pero el predicho del padre, se estarían comparando cuantiles de distribuciones de ingresos distintos pudiendo sesgar la medida de movilidad ascendente considerada.
} 
(5), en los valores promedios de las variables explicativas, un incremento del $1 \%$ en el gasto público social aumenta en 0.010 la probabilidad de ascender un percentil en la distribución del ingreso. El efecto es levemente superior para el gasto público social en salud, promoción social y educación (GPSCH3) que incrementa la probabilidad de movilidad ascendente en 0.012 ante un aumento del $1 \%$ en esta medida del gasto. Como este incremento del gasto puede considerarse demasiado pequeño, a fin de tener una idea más clara de la magnitud del efecto del gasto en la probabilidad de los hijos de ubicarse en un percentil superior al de su padre, se estimaron las probabilidades predichas para tres niveles del gasto: bajo, medio y alto (en el valor promedio del gasto público correspondiente a cada tercil de la distribución del gasto).

\section{Cuadro 10. Efectos del gasto público sobre la probabilidad de movilidad ascendente. Modelo probit.}

\begin{tabular}{|c|c|c|c|c|}
\hline \multirow{2}{*}{$\begin{array}{c}\text { Medidas de gasto público } \\
\text { consolidado per cápita (en log) }\end{array}$} & \multicolumn{2}{|c|}{ "Modelo 1} & "Modelo 2 & "Modelo 3 \\
\hline & Coef & Ef. Marg. & Ef. Marg. & Ef. Marg. \\
\hline Total & $\begin{array}{c}0.061 \\
(0.100)\end{array}$ & $\begin{array}{l}0.0002 \\
(0.038)\end{array}$ & 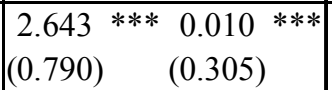 & $\begin{array}{cccc}2.241 & * * & 0.009 & * * \\
(0.949) & & (0.363) & \end{array}$ \\
\hline Social total & $\left(\begin{array}{c}0.192 \\
(0.121)\end{array}\right.$ & $\begin{array}{c}0.001 \\
(0.046)\end{array}$ & 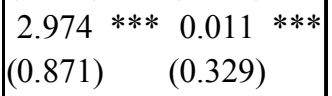 & 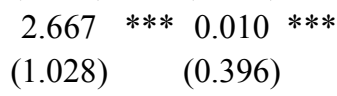 \\
\hline GPSCH1 & $\left(\begin{array}{l}0.085 \\
(0.093)\end{array}\right.$ & $\begin{array}{l}0.0003 \\
(0.036)\end{array}$ & 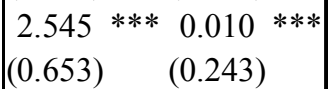 & \begin{tabular}{ccc}
2.092 & $* *$ & $0.008 \quad * *$ \\
$(1.003)$ & \multicolumn{2}{c}{$(0.386)$}
\end{tabular} \\
\hline GPSCH2 & $\begin{array}{c}0.105 \\
(0.096)\end{array}$ & $\begin{array}{l}0.0004 \\
(0.037)\end{array}$ & 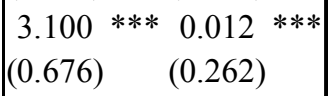 & $\begin{array}{ccc}2.945 & * * * & 0.011 \\
(0.861) & (0.328)\end{array}$ \\
\hline GPSCH3 & $\left(\begin{array}{c}0.097 \\
(0.098)\end{array}\right.$ & $\begin{array}{l}0.0004 \\
(0.037)\end{array}$ & 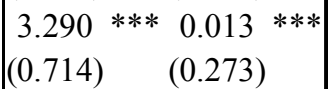 & $\begin{array}{ccc}3.067 & * * * & 0.012 \\
(0.995) & (0.378)\end{array}$ \\
\hline GPSCH4 & $\begin{array}{c}0.061 \\
(0.099)\end{array}$ & $\begin{array}{l}0.0002 \\
(0.038)\end{array}$ & $\mid \begin{array}{lc}3.224 * * * & 0.012 \\
(0.658) & (0.261)\end{array}$ & $\begin{array}{ccc}2.928 & * * * & 0.011 * * * \\
(0.928) & (0.349)\end{array}$ \\
\hline
\end{tabular}

Fuente: Elaboración propia sobre la base de varias fuentes de información. Ver la sección de datos para más detalles.

Nota: $* * * p$-value $<0.01, * *$ p-value $<0.05, *$ p-value $<0.1$. Entre paréntesis se reportan los errores estándares agrupados a nivel provincial. Ver la nota del Cuadro 3 con la descripción de la especificación de cada modelo. Los efectos marginales fueron computados en los valores promedios de las variables explicativas.

Como surge del Cuadro 11, si se considera la especificación más completo del modelo probit estimado, un incremento del gasto público total per cápita de $\$ 1917$ (valor promedio en el tercil más bajo) a \$2905 (valor promedio en el tercil más alto), esto es, en un 52\%, aumenta la probabilidad predicha de movilidad ascendente de 0.441 a 0.777 , es decir, en un $43.3 \%$. En tanto que, un crecimiento del gasto público social y del GPSCH3 en un porcentaje relativamente similar incrementa esta probabilidad en un $47.5 \%$ y $52.6 \%$, respectivamente. Conforme con los intervalos de confianza, las 
diferencias entre estas probabilidades predichas son estadísticamente significativas, al menos a un nivel de significancia del 5\%.

Cuadro 11. Probabilidades predichas de movilidad ascendente por nivel de gasto.

\begin{tabular}{|c|c|c|c|c|c|c|c|c|c|c|}
\hline \multirow{3}{*}{ Medida de gasto } & \multirow{3}{*}{ Modelo } & \multicolumn{3}{|c|}{ Probabilidad predicha } & \multicolumn{6}{|c|}{ Diferencias en la probabilidad según nivel de gasto } \\
\hline & & \multirow{2}{*}{ Gasto bajo } & \multirow{2}{*}{ Gasto medio } & \multirow{2}{*}{ Gasto alto } & \multicolumn{2}{|c|}{ Bajo - medio } & \multicolumn{2}{|c|}{ Medio - alto } & \multicolumn{2}{|c|}{ Bajo-alto } \\
\hline & & & & & En nivel & $E n \%$ & En nivel & $E n \%$ & En nivel & $E n \%$ \\
\hline \multirow[t]{3}{*}{ Total } & 1 & $\begin{array}{c}0.612 \\
0.597-0.628\end{array}$ & $\begin{array}{c}0.615 \\
0.606-0.623\end{array}$ & $\begin{array}{c}0.622 \\
0.607-0.638\end{array}$ & -0.002 & $-0.4 \%$ & -0.008 & $-1.3 \%$ & -0.010 & $-1.6 \%$ \\
\hline & 2 & 0.405 & 0.498 & 0.800 & -0.093 & $-23.0 \%$ & -0.302 & $-60.7 \%$ & -0.396 & $-49.4 \%$ \\
\hline & 3 & $\begin{array}{c}0.269-0.540 \\
0.441 \\
0270-0612\end{array}$ & $\begin{array}{c}0.419-0.511 \\
0.520 \\
0.422-0617\end{array}$ & $\left|\begin{array}{c}0.703-0.898 \\
0.777 \\
0.647-0.907\end{array}\right|$ & -0.079 & $-17.9 \%$ & -0.257 & $-49.5 \%$ & -0.336 & $-43.3 \%$ \\
\hline \multirow[t]{3}{*}{$\begin{array}{l}\text { Servicios } \\
\text { sociales }\end{array}$} & 1 & $\begin{array}{c}0.605 \\
0.589-0.620\end{array}$ & $\begin{array}{c}0.613 \\
0.609-0.618\end{array}$ & $\begin{array}{c}0.637 \\
0.613-0.662\end{array}$ & -0.009 & $-1.4 \%$ & -0.024 & $-3.9 \%$ & -0.033 & $-5.1 \%$ \\
\hline & 2 & $\begin{array}{c}0.420 \\
0.298-0.543\end{array}$ & $\begin{array}{c}0.557 \\
0.518-0.596\end{array}$ & $\mid \begin{array}{c}0.866 \\
0.757-0.975\end{array}$ & -0.137 & $-32.5 \%$ & -0.309 & $-55.5 \%$ & -0.446 & $-51.5 \%$ \\
\hline & 3 & $\begin{array}{c}0.444 \\
0.291-0.598\end{array}$ & $\begin{array}{c}0.566 \\
0.517-0.615\end{array}$ & $\mid \begin{array}{c}0.847 \\
0.699-\end{array}$ & -0.122 & $-27.4 \%$ & -0.281 & $-49.7 \%$ & -0.403 & $-47.5 \%$ \\
\hline \multirow[t]{3}{*}{ GPSCH1 } & 1 & $\begin{array}{c}0.610 \\
0.595-0.625\end{array}$ & $\begin{array}{c}0.613 \\
0.605-0.622\end{array}$ & $\begin{array}{c}0.624 \\
0.610-\quad 0.639\end{array}$ & -0.003 & $-0.5 \%$ & -0.011 & $-1.8 \%$ & -0.014 & $-2.3 \%$ \\
\hline & 2 & $\begin{array}{c}0.407 \\
0.288-0.525\end{array}$ & $\begin{array}{c}0.500 \\
0.432-0.568\end{array}$ & $\begin{array}{c}0.794 \\
0.710-\quad 0.878\end{array}$ & -0.093 & $-23.0 \%$ & -0.294 & $-58.9 \%$ & -0.388 & $-48.8 \%$ \\
\hline & 3 & $\begin{array}{c}0.447 \\
0.263-0.630\end{array}$ & $\begin{array}{c}0.524 \\
0.420-\quad 0.627\end{array}$ & $\begin{array}{c}0.768 \\
0.630-0.905\end{array}$ & -0.077 & $-17.2 \%$ & -0.244 & $-46.6 \%$ & -0.321 & $-41.8 \%$ \\
\hline \multirow[t]{3}{*}{ GPSCH2 } & 1 & $\begin{array}{c}0.609 \\
0.595-0.623\end{array}$ & $\begin{array}{c}0.614 \\
0.607-0.620\end{array}$ & $\begin{array}{c}0.627 \\
0.610-\quad 0.645\end{array}$ & -0.004 & $-0.7 \%$ & -0.014 & $-2.2 \%$ & -0.018 & $-2.8 \%$ \\
\hline & 2 & $\begin{array}{c}0.382 \\
0.278-0.487\end{array}$ & $\begin{array}{c}0.511 \\
0.461-0.561\end{array}$ & $\begin{array}{c}0.852 \\
0.774-0.931\end{array}$ & -0.128 & $-33.6 \%$ & -0.342 & $-67.0 \%$ & -0.470 & $-55.2 \%$ \\
\hline & 3 & $\begin{array}{c}0.397 \\
0.258-0.535\end{array}$ & $\begin{array}{c}0.519 \\
0.452-0.585\end{array}$ & $\begin{array}{c}0.844 \\
0.737-0.951\end{array}$ & -0.122 & $-30.7 \%$ & -0.326 & $-62.8 \%$ & -0.447 & $-53.0 \%$ \\
\hline \multirow[t]{3}{*}{\begin{tabular}{|l|} 
GPSCH3 \\
\end{tabular}} & 1 & $\begin{array}{c}0.611 \\
0.597-0.624\end{array}$ & $\begin{array}{c}0.615 \\
0.609-0.620\end{array}$ & \begin{tabular}{|c|}
0.627 \\
$0.609-\quad 0.645$
\end{tabular} & -0.004 & $-0.7 \%$ & -0.012 & $-2.0 \%$ & -0.016 & $-2.6 \%$ \\
\hline & 2 & $\begin{array}{c}0.387 \\
0.278-0.495\end{array}$ & $\begin{array}{c}0.521 \\
0.473-0.569\end{array}$ & $\mid \begin{array}{c}0.868 \\
0.784-0.952\end{array}$ & -0.135 & $-34.8 \%$ & -0.346 & $-66.4 \%$ & -0.481 & $-55.4 \%$ \\
\hline & 3 & $\begin{array}{c}0.406 \\
0.252-0.560\end{array}$ & $\begin{array}{c}0.531 \\
0.465-0.597\end{array}$ & $\begin{array}{c}0.856 \\
0.730-0.981\end{array}$ & -0.125 & $-30.9 \%$ & -0.325 & $-61.2 \%$ & -0.450 & $-52.6 \%$ \\
\hline \multirow[t]{3}{*}{ GPSCH4 } & 1 & $\begin{array}{c}0.613 \\
0.601-0.626\end{array}$ & $\begin{array}{c}0.616 \\
0.611-0.621\end{array}$ & 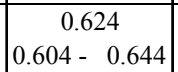 & -0.002 & $-0.4 \%$ & -0.008 & $-1.3 \%$ & -0.011 & $-1.7 \%$ \\
\hline & 2 & $\begin{array}{c}0.403 \\
0.307-0.498\end{array}$ & $\begin{array}{c}0.532 \\
0.491-0.573\end{array}$ & $\begin{array}{c}0.875 \\
0.797-0.954\end{array}$ & -0.130 & $-32.2 \%$ & -0.343 & $-64.5 \%$ & -0.473 & $-54.0 \%$ \\
\hline & 3 & $\begin{array}{c}0.425 \\
0.289-0.562 \\
\end{array}$ & \begin{tabular}{|c}
0.543 \\
$0.487-0.599$ \\
\end{tabular} & $\begin{array}{c}0.859 \\
0.738-\quad 0.980\end{array}$ & -0.117 & $-27.6 \%$ & -0.316 & $-58.2 \%$ & -0.433 & $-50.5 \%$ \\
\hline
\end{tabular}

Fuente: Elaboración propia sobre la base de varias fuentes de información. Ver la sección de datos para más detalles.

Nota: Bajo las probabilidades predichas se reportan los intervalos de confianza al $95 \%$, computados con el método delta. Ver la nota del Cuadro 3 con la descripción de la especificación de cada modelo.

Por otra parte, cuando se considera movimientos ascendentes de mayor magnitud, se observa que el gasto público tiene un efecto significativo para $0<\tau<0.20$. Es decir, la probabilidad de superar el percentil del padre en magnitudes mayores a cero pero menores a 0.20 , son más elevadas para los hijos que experimentaros niveles más altos de gasto público en su juventud. Esto significa que el gasto no sólo tiene un efecto positivo en la probabilidad de los hijos de superar el percentil de sus padres sino también en la de abandonar el decil más bajo en el que se encontraban sus padres. Este 
resultado puede observarse en el Cuadro A4 donde se reportan las estimaciones del modelo probit para $\tau=0.10$ que sugieren que el gasto público total, el gasto público social y casi todas las medidas de inversión pública en capital humano consideradas (salvo el GPSCH1) tienen un efecto positivo en la probabilidad de los hijos de superar el decil en el que se encontraban sus padres. Sin embargo, para valores mayores de $\tau$, concretamente para $\tau=0.20$ no se observan efectos significativos del gasto en la probabilidad de movilidad ascendente. Por lo tanto, las estimaciones sugieren que el gasto público tiene un impacto limitado en la movilidad intergeneracional ascendente, concretamente parece no tener efectos en la probabilidad de que los hijos superen el quintil en el que se encontraba sus padres. No obstante, cuando se consideran movimientos ascendentes de menor magnitud se obtienen efectos positivos $y$ significativos. Este resultado es consistente con los impactos relativamente modestos del gasto público sobre los niveles promedios de persistencia intergeneracional medidos a partir de la EII. No obstante, las estimaciones presentadas en este apartado deben ser interpretadas con precaución puesto que las limitaciones de los datos utilizados dificultan, en general, el análisis de probabilidades de transición del ingreso ${ }^{168}$.

\subsection{Análisis de robustez}

La extensión de la serie histórica del GPC por ubicación geográfica no permite examinar el efecto del gasto público que experimentaron durante la niñez o adolescencia los adultos de la muestra principal. Sin embargo, varios estudios han reportado mayores tasas de retorno de intervenciones en capital humano en la primera infancia que intervenciones en etapas posteriores del ciclo de vida durante la adolescencia, juventud o adultez (Duncan y Brook-Gunn, 1997; Duncan et al., 1998, Bernal y Camacho, 2010). Esto sucede, entre otras razones, porque la capacidad de los seres humanos para aprender durante la primera infancia es mucho mayor y su conjunto de habilidades básicas es mucho más maleable que en los años posteriores (Heckman, 2000 y 2008). La evidencia empírica sugiere que la habilidad cognitiva se forma relativamente temprano en el ciclo de vida, está prácticamente determinada en su totalidad entre los 8

\footnotetext{
${ }^{168}$ Véase para más detalles la sección 3 del Capítulo 2.
} 
y 14 años de edad y se vuelve mucho menos maleable con el paso del tiempo (Carneiro y Heckman, 2003). No obstante, otra evidencia sugiere que el momento de la inversión no es relevante o que es más importante cuando se mide cerca del resultado de interés (Mayer, 2002).

A fin de examinar el efecto de la edad y el período temporal en la que se mide la inversión pública en el capital humano de los hijos se utilizan en esta sección los datos sobre el gasto público provincial per cápita (GPPpc) por finalidad y función para el período 1993-2012 que provienen de la Dirección de Análisis del Gasto Público y Programas Sociales (DAGPyPS) del MECON. Estos datos permiten obtener una medida del gasto público durante la adolescencia de los hijos adultos de la muestra principal. Conforme se observa en el Cuadro A1, los hijos de 25 a 34 años observados durante 2003-2012, tenían entre 15 y 17 años en el período 1993-2012. Aunque existen datos sobre el gasto público nacional para el mismo período, no están distribuidos geográficamente y no se dispone de la información necesaria para hacer esta distribución de forma similar a como se la realizó para el período 1998-2008, en particular no se cuenta con la apertura programática del gasto nacional por finalidad y función, ni con indicadores provinciales apropiados ${ }^{169}$. No obstante, una elevada proporción del GPC particularmente en las partidas más directamente relacionadas con el capital humano de los hijos es ejecutado por los gobiernos provinciales. Así, en promedio, durante 1998-2008, el 40.4\% del GPC es ejecutado por las provincias. En particular, el $80 \%$ del GPC en educación y cultura y más del $90 \%$ del gasto en educación básica es ejecutado por los gobiernos provinciales. Esto refleja la responsabilidad de las provincias en el financiamiento y provisión de los servicios de educación básica en Argentina como resultado de un proceso de descentralización ocurrido principalmente durante los 90 . No obstante, el primer proceso de transferencia de servicios educativos de la Nación a las provincias se produjo en 1978. Mediante las leyes $\mathrm{N}^{\circ} 21.809 \mathrm{y} \mathrm{N}^{\circ} 21.810$ se facultó al gobierno nacional a transferir a las provincias

\footnotetext{
169 Por ejemplo, no hay información disponible sobre la cantidad de alumnos por nivel educativo y provincia para los años 1993 ni 1995 porque el Ministerio de Educación de la Nación no realizó relevamientos ni elaboración de información estadística para el período 1989-1993 y para el año1995 (Ferreres, 2010). No obstante, este constituye un indicador relevante para distribuir el gasto público nacional en educación básica en función de sus principales beneficiarios. De la misma forma, un indicador que podría utilizarse para distribuir el gasto público nacional en atención de la salud entre sus beneficiarios sería la cantidad de población que no cuenta con cobertura de salud. Sin embargo, no hay bases de datos que permitan computar este indicador a nivel provincial para el período 1993-1996.
} 
los establecimientos de enseñanza preprimaria y primaria que estaban a cargo de la nación. Tres años más tarde, el proceso se completó con la transferencia de los establecimientos para adultos (Curcio et al., 2009). Luego, en 1992, con la Ley $\mathrm{N}^{\circ}$ 24.049, se dispuso la transferencia de la administración y financiamiento de los servicios educativos que aún estaban en poder del gobierno Nacional a los gobiernos provinciales y a la Ciudad de Buenos Aires (Cetrángolo y Gatto, 2002). En 1993, mediante la Ley Federal de Educación $N^{\circ} 24.185$ se dispone una serie de reformas que apoyan la redefinición de los roles administrativos y responsabilidades de financiamiento del sistema educativo para cada nivel de gobierno. Las provincias fueron designadas como las principales administradoras de la educación pública pre universitaria y de la formación inicial docente en todo el país (Curcio et al., 2009). Por otra parte, el 69\% del GPC en atención médica es ejecutado por las provincias. Esto responde, en gran medida a la modificación introducida en 1992, a través de la Ley $\mathrm{N}^{\circ}$ 24.061 que reglamentó la transferencia, a la jurisdicción de las respectivas provincias y de la ciudad de Buenos Aires, de la administración y financiamiento de 20 hospitales e institutos dependientes del gobierno nacional. Durante la década de 1990 también se descentralizaron otras áreas de la política social: vivienda, programas nutricionales y otros programas asistenciales. Esto se refleja en que el 54\% del GPC en promoción y asistencia social total, el 91\% del gasto en nutrición y alimentación, el $82 \%$ del gasto en deporte y recreación y $77 \%$ del gasto en vivienda son ejecutados por las provincias (ver Gráfico A1). Por otra parte, este ejercicio permite analizar también en qué medida el gasto público provincial, sin el gasto nacional ni el municipal, es efectivo para reducir los niveles de persistencia socioeconómica intergeneracional.

En los Cuadros 12 y 13 se presentan los resultados de la estimación de tres especificaciones del modelo (1) para el GPPpc total, social y para tres medidas del GPSCH que pueden computarse a partir de la apertura por finalidad y función disponible para el GPPpc: el GPSCH1 (gasto público en salud, promoción social, educación, vivienda y agua), el GPSCH2 (gasto público en salud, promoción social, educación y agua) y el GPSCH3 (gasto público en salud, promoción social y educación). En todas las especificaciones el ingreso estimado del padre tiene un efecto positivo y estadísticamente significativo al $1 \%$ en el ingreso del hijo. Por su parte, todas las medidas de gasto público consideradas presentan un efecto positivo en el ingreso y en la educación de los hijos. Sin embargo, el efecto positivo del gasto público en el 
ingreso de los hijos no es significativo en el caso del GPPpc total, social y el GPSCH $1{ }^{170}$. Los términos de interacción entre el GPPpc y el ingreso o la educación del padre tienen el signo esperado, siendo negativos y estadísticamente significativos para las tres medidas de inversión pública en capital humano consideradas (GPSCH1, GPSCH2, GPSCH3). A su vez, tanto en el caso de la EII como en el caso del CPE, el efecto del gasto público se incrementa levemente en el caso de las medidas que se consideran más directamente beneficiosas para los niños adolescentes. Así, mientras un incremento del 1\% en el GPSCH1 reduce la EII en 0.077 p.p. y el CPE en 0.081 p.p., un aumento del GPSCH3 en el mismo porcentaje disminuye estas medidas de persistencia intergeneracional en 0.105 p.p. y en 0.097 p.p., respectivamente. No obstante, las diferencias en los efectos de las tres medidas de inversión pública en capital humano no son estadísticamente significativas.

Por otra parte, el efecto del GPPpc tanto en la EII como en el CPE parecen ser menores que el del GPCpc. Así, si se considera la especificación más completa del modelo y la tercera medida de inversión pública en capital humano (GPSCH3), un incremento del $1 \%$ en el gasto en salud, promoción social y educación reducen la EII en 0.170 p.p. y el CPE, en 0.166 cuando se considera el GPCpc pero en 0.105 p.p. y 0.097 p.p, en el caso del GPPpc, respectivamente. Además, ambas diferencias son estadísticamente significativas. Este es un resultado esperable dado que el GPCpc es mayor, por definición, que el GPPpc, debe tenerse en cuenta también que la edad promedio de los hijos en la que se mide el primero (18 a 24 años) es mayor que la edad en la que se mide el segundo (15 a 17 años). Por tanto, si conforme surge de algunos estudios, las tasas de retorno de las intervenciones en capital humano en la niñez o adolescencia son mayores que las de intervenciones en etapas posteriores del ciclo de vida, el efecto del GPPpc podría ser igual o mayor que el del GPCpc.

\footnotetext{
${ }^{170}$ No obstante, cuando se considera el nivel del gasto público per cápita (estandarizado) en lugar de su logaritmo en las tres especificaciones del modelo analizadas, los efectos principales así como los términos de interacción resultan estadísticamente significativos para todas las medidas de gasto público. Una explicación posible de este resultado es que la transformación logarítmica del gasto público provincial disminuye su variabilidad y también su correlación con el ingreso y la educación de los hijos.
} 


\section{Cuadro 12. Efecto del gasto público provincial per cápita sobre la elasticidad intergeneracional del ingreso}

\begin{tabular}{|c|c|c|c|c|}
\hline $\begin{array}{l}\text { Medidas } \\
\text { GPPpc }\end{array}$ & Variables de interés & Mod. 1 & Mod. 2 & Mod. 3 \\
\hline Total & $\begin{array}{l}\text { Log del Ingreso del padre } \\
\text { Log Gasto público per cápita } \\
\text { Log del Ingreso del padre x Log Gasto público } \\
\text { per cápita }\end{array}$ & $\begin{array}{c}0.797 * * * \\
(0.257) \\
0.198 \\
(0.209) \\
-0.024 \\
(0.039)\end{array}$ & $\begin{array}{c}0.915 * * * \\
(0.277) \\
0.182 \\
(0.201) \\
-0.041 \\
(0.042)\end{array}$ & $\begin{array}{c}0.957 * * * \\
(0.305) \\
0.191 \\
(0.227) \\
-0.048 \\
(0.047)\end{array}$ \\
\hline \begin{tabular}{|l|} 
Servicios \\
Sociales
\end{tabular} & $\begin{array}{l}\text { Log del Ingreso del padre } \\
\text { Log Gasto público per cápita } \\
\text { Log del Ingreso del padre x Log Gasto público } \\
\text { per cápita }\end{array}$ & $\begin{array}{c}0.910 * * * \\
(0.206) \\
0.286 \\
(0.183) \\
-0.043 \\
(0.034) \\
\end{array}$ & $\begin{array}{c}1.005 * * * \\
(0.231) \\
0.218 \\
(0.175) \\
-0.058 \\
(0.038) \\
\end{array}$ & $\begin{array}{c}1.083 * * * \\
(0.270) \\
0.242 \\
(0.209) \\
-0.070 \\
(0.044)\end{array}$ \\
\hline GPSCHN1 & $\begin{array}{l}\text { Log del Ingreso del padre } \\
\text { Log Gasto público per cápita } \\
\text { Log del Ingreso del padre x Log Gasto público } \\
\text { per cápita }\end{array}$ & $\begin{array}{c}0.884 * * * \\
(0.182) \\
0.255 \\
(0.182) \\
-0.040 \\
(0.030)\end{array}$ & $\begin{array}{c}1.015^{* * *} \\
(0.181) \\
0.239 \\
(0.160) \\
-0.062^{*} \\
(0.030)\end{array}$ & $\begin{array}{c}1.109 * * * \\
(0.209) \\
0.272 \\
(0.188) \\
-0.077 * * \\
(0.035)\end{array}$ \\
\hline GPSCHN2 & $\begin{array}{l}\text { Log del Ingreso del padre } \\
\text { Log Gasto público per cápita } \\
\begin{array}{l}\text { Log del Ingreso del padre x Log Gasto público } \\
\text { per cápita }\end{array}\end{array}$ & $\begin{array}{c}0.984 * * * \\
(0.168) \\
0.329 * \\
(0.159) \\
-0.057 * \\
(0.028)\end{array}$ & $\begin{array}{c}1.112 * * * \\
(0.164) \\
0.325 * * \\
(0.140) \\
-0.078 * * * \\
(0.027)\end{array}$ & $\begin{array}{c}1.216^{* * *} \\
(0.185) \\
0.370^{* *} \\
(0.158) \\
-0.096 * * * \\
(0.031)\end{array}$ \\
\hline \begin{tabular}{|l|} 
GPSCHN3 \\
\end{tabular} & $\begin{array}{l}\text { Log del Ingreso del padre } \\
\text { Log Gasto público per cápita } \\
\begin{array}{l}\text { Log del Ingreso del padre x Log Gasto público } \\
\text { per cápita }\end{array}\end{array}$ & $\begin{array}{c}1.044 * * * \\
(0.161) \\
0.408 * * \\
(0.147) \\
-0.067 * * \\
(0.027)\end{array}$ & $\begin{array}{c}1.164 * * * \\
(0.156) \\
0.385^{* * *} \\
(0.131) \\
-0.087 * * * \\
(0.026)\end{array}$ & $\begin{array}{c}1.269 * * * \\
(0.171) \\
0.427 * * * \\
(0.143) \\
-0.105 * * * \\
(0.029)\end{array}$ \\
\hline
\end{tabular}

Fuente: Elaboración propia sobre la base de varias fuentes de información. Ver la sección de datos para más detalles.

Nota: $* * * p$-value $<0.01, * * p$-value $<0.05, * p$-value $<0.1$. Entre paréntesis se reportan los errores estándares agrupados a nivel provincial. El Modelo 1 incluye además de las variables de interés, controles por el sexo del hijo, la edad del hijo y de los padres (polinomio cuártico), efectos fijos por provincia de residencia, dummies por año y dummies por cohorte, el Modelo 2 suma a las anteriores un conjunto de variables de control por características provinciales existentes durante la juventud de los hijos (los recursos tributarios provinciales, la tasa de desocupación, el nivel de desigualdad, la tasa de crecimiento del PBG, la tasa de pobreza por NBI, la tasa de asistencia a cada nivel educativo, los años de educación promedio de la PET, la cantidad total de población, la tasa de mortalidad infantil (por mil nacidos vivos), la tasa de mortalidad total (por mil habitantes) y el porcentaje de la población sin acceso a agua corriente, el partido político del gobernador de cada provincia en relación con el del presidente de turno) y la proporción del gasto público per cápita destinado a otras partidas, salvo en el modelo estimado para el GPCpc total y el Modelo 3, incorpora al anterior los términos de interacción entre las dummies locales y temporales.

Cuando se consideran por separado las distintas categorías del gasto público provincial social per cápita ${ }^{171}$ se observa que los gastos en salud, en promoción y asistencia social y en educación total tienen efectos positivos sobre el ingreso y la educación de los hijos (ver Cuadro A5 y A6 del Anexo). Además todos los términos de interacción de estas tres categorías del gasto tienen el signo negativo esperado y resultan

\footnotetext{
${ }^{171}$ Para el GPP no se dispone de la misma apertura por finalidad y función que para el GPC por eso, se consideran en este análisis menos categorías del gasto público social.
} 
estadísticamente significativos sugiriendo que un incremento del gasto público provincial en estas categorías reduce la persistencia intergeneracional de ingresos así como la educativa. Y, a excepción del gasto público en salud, sus efectos individuales en la EII y en el CPE no son significativamente distintos de los estimados para las mismas categorías del GPC. Esto probablemente responde a la elevada participación de los gobiernos provinciales en la ejecución del GPC en estas partidas (ver Gráfico A1).

\section{Cuadro 13. Efecto del gasto público provincial per cápita sobre el coeficiente de persistencia educativa intergeneracional}

\begin{tabular}{|c|c|c|c|c|}
\hline $\begin{array}{l}\text { Medidas } \\
\text { GPPpc }\end{array}$ & Variables de interés & Mod. 1 & Mod. 2 & Mod. 3 \\
\hline Total & $\begin{array}{l}\text { Educación del padre } \\
\text { Log Gasto público per cápita } \\
\begin{array}{l}\text { Educación del padre x Log Gasto público per } \\
\text { cápita }\end{array}\end{array}$ & $\begin{array}{c}0.656^{* * *} \\
(0.205) \\
1.406 * * \\
(0.623) \\
-0.041 \\
(0.032) \\
\end{array}$ & $\begin{array}{c}0.652 * * * \\
(0.207) \\
0.990 * * \\
(0.413) \\
-0.040 \\
(0.032) \\
\end{array}$ & $\begin{array}{c}0.674 * * * \\
(0.210) \\
0.814 \\
(0.486) \\
-0.044 \\
(0.032) \\
\end{array}$ \\
\hline $\begin{array}{l}\text { Servicios } \\
\text { Sociales }\end{array}$ & $\begin{array}{l}\text { Educación del padre } \\
\text { Log Gasto público per cápita } \\
\begin{array}{l}\text { Educación del padre x Log Gasto público per } \\
\text { cápita }\end{array}\end{array}$ & $\begin{array}{c}0.883 * * * \\
(0.286) \\
1.558 * * \\
(0.649) \\
-0.079 \\
(0.046)\end{array}$ & $\begin{array}{c}0.881 * * * \\
(0.287) \\
0.660 \\
(0.440) \\
-0.079 \\
(0.046)\end{array}$ & $\begin{array}{c}0.898 * * * \\
(0.280) \\
0.440 \\
(0.500) \\
-0.082 * \\
(0.045)\end{array}$ \\
\hline GPSCHN1 & $\begin{array}{l}\text { Educación del padre } \\
\text { Log Gasto público per cápita } \\
\begin{array}{l}\text { Educación del padre x Log Gasto público per } \\
\text { cápita }\end{array}\end{array}$ & $\begin{array}{c}0.880 * * * \\
(0.281) \\
2.012 * * \\
(0.753) \\
-0.082 * \\
(0.047) \\
\end{array}$ & $\begin{array}{c}0.863 * * * \\
(0.273) \\
1.537 * * \\
(0.596) \\
-0.079 * \\
(0.046) \\
\end{array}$ & $\begin{array}{c}0.872^{* * *} \\
(0.266) \\
1.130 \\
(0.663) \\
-0.081^{*} \\
(0.045) \\
\end{array}$ \\
\hline GPSCHN2 & $\begin{array}{l}\text { Educación del padre } \\
\text { Log Gasto público per cápita } \\
\begin{array}{l}\text { Educación del padre x Log Gasto público per } \\
\text { cápita }\end{array}\end{array}$ & $\begin{array}{c}0.958 * * * \\
(0.288) \\
1.980 * * \\
(0.761) \\
-0.095 * \\
(0.048)\end{array}$ & $\begin{array}{c}0.935 * * * \\
(0.277) \\
1.487 * * \\
(0.552) \\
-0.092 * \\
(0.047)\end{array}$ & $\begin{array}{c}0.941 * * * \\
(0.269) \\
1.151 * \\
(0.614) \\
-0.093 * \\
(0.045)\end{array}$ \\
\hline GPSCHN3 & $\begin{array}{l}\text { Educación del padre } \\
\text { Log Gasto público per cápita } \\
\begin{array}{l}\text { Educación del padre x Log Gasto público per } \\
\text { cápita }\end{array}\end{array}$ & $\begin{array}{c}0.975 * * * \\
(0.275) \\
2.086 * * \\
(0.781) \\
-0.099 * * \\
(0.046)\end{array}$ & $\begin{array}{c}0.957 * * * \\
(0.266) \\
1.569 * * \\
(0.571) \\
-0.096 * * \\
(0.045)\end{array}$ & $\begin{array}{c}0.963 * * * \\
(0.258) \\
1.184 * \\
(0.646) \\
-0.097 * * \\
(0.043)\end{array}$ \\
\hline
\end{tabular}

Fuente: Elaboración propia sobre la base de varias fuentes de información. Ver la sección de datos para más detalles.

Nota: $* * * p$-value $<0.01$, ** p-value $<0.05, * p$-value $<0.1$. Entre paréntesis se reportan los errores estándares agrupados a nivel provincial. Ver la nota del Cuadro 12 con la descripción de la especificación de cada modelo.

Un resultado llamativo es la falta de significancia estadística del efecto del gasto en educación básica tanto en el ingreso y educación de los hijos como en las medidas de 
persistencia socioeconómica entre padres e hijos. No obstante, conforme se explicó previamente, esto podría nuevamente estar relacionado con la edad en la que se mide la inversión pública en educación. En efecto, la medida de inversión pública considerada es el GPP promedio que experimentaron los hijos adultos cuando tenían entre 15 y 17 años. Sin embargo, el GPP en educación básica comprende, además del gasto en educación media, el gasto en educación elemental y educación primaria que no benefician directamente a los hijos en su adolescencia sino durante su niñez. No obstante, otra explicación factible para la falta de significancia del gasto en educación básica es la existencia de deficiencias en la calidad de los servicios educativos públicos. Asimismo, este resultado podría estar relacionado también con la creciente segregación escolar que se observa en Argentina durante el período 1992-2009 según los resultados obtenidos por Gasparini et al. (2012) y Jaume (2013). En efecto, como advierte Krüger (2013) el hecho de que los jóvenes se concentren en determinados centros según su nivel socioeconómico incide en la desigualdad de logros educativos en el nivel medio argentino. De esta forma, la segregación implica que los estudiantes de origen social desfavorecido se encuentran en desventaja por su menor capital económico y cultural y por asistir a escuelas en las que no pueden beneficiarse del intercambio con pares de distintos segmentos sociales. Adicionalmente, la integración social en la escuela no sólo afecta los resultados educativos inmediatos sino también puede incidir en la cantidad de educación demandada luego del egreso y en la inserción laboral (Brunello y Checchi, 2006; Sendón, 2005).

\section{Conclusiones}

El objetivo de esta investigación fue determinar el efecto que tiene sobre la MI en Argentina, el gasto público realizado por los tres niveles de gobierno (nacional, provincial y municipal) que modificaron las inversiones públicas en el capital humano de los niños y adolescentes durante el período 1998-2008. Específicamente, se buscó evaluar si el gasto público cumple con uno de los objetivos centrales de la política social que es incrementar la movilidad económica intergeneracional y la igualdad de oportunidades, disminuyendo el impacto del origen socioeconómico en el ingreso obtenido en la adultez. Asimismo, se examinó si la brecha de movilidad observada entre 
los hijos que provienen de trasfondo familiares socioeconómicamente distintos es menor entre las cohortes que experimentaron mayores niveles de gasto público durante su adolescencia y juventud que en aquellas para las cuales el gasto público fue menor.

Los resultados obtenidos sugieren que el gasto público tiene el efecto esperado, incrementando la movilidad intergeneracional tanto del ingreso como la educativa. Sin embargo, no todas las categorías de gasto son igualmente efectivas para conseguir este resultado. Así, se observa una disminución mayor en la persistencia socioeconómica entre generaciones, medida por la EII y por el CPE cuando se incrementan las medidas del GPC más directamente relacionadas con el capital humano de los niños y adolescentes como las que surgen de agregar el gasto en salud, promoción social y educación.

Si bien las estimaciones anteriores surgen de explotar la variabilidad temporal (entre cohortes de nacimiento) del gasto público, controlando por un amplio conjunto de características individuales y principalmente, locales de las provincias de residencia de los hijos podrán estar sesgada por la presencia de factores locales inobservables que varían en el tiempo. Una forma de corregir este problema, bajo determinados supuestos, es mediante la estrategia de diferencias en diferencias que permite, además examinar el efecto del gasto público en las brechas de movilidad intergeneracional según nivel educativo de los padres. En general, los resultados obtenidos a partir de esta técnica confirman los anteriores. No obstante, debe señalarse que, desde el punto de vista económico, la magnitud de los efectos estimados del gasto público sobre la movilidad intergeneracional son en general modestos, sugiriendo que se requieren de elevados incrementos en el gasto para disminuir significativamente las medidas de persistencia intergeneracional consideradas o incrementar las probabilidades de movilidad ascendente de los hijos.

Estos hallazgos deben interpretarse con precaución debido a las limitaciones informativas existentes, en particular, el gasto público consolidado por provincia sólo está disponible para el período 1998-2008. Por tanto, las estimaciones podrían cambiar al considerar los niveles de gasto público para otros períodos y/o al cambiar la edad en el que se observa el ingreso familiar de los hijos adultos. En particular, conforme fue señalado previamente, varios estudios han reportado mayores tasas de retorno de intervenciones en capital humano en la primera infancia. Por tanto, es probable que los 
efectos del gasto aquí estimado se incrementen al medir las inversiones públicas en el capital humano durante la niñez de los hijos adultos. No obstante, el análisis de robustez realizado utilizando el gasto público provincial para el período 1993-2004, que permite medir la inversión pública en capital humano ejecutada por los gobiernos locales durante la adolescencia de los hijos (concretamente cuando tenían de 15 a 17 años), sugiere que las principales conclusiones no se modifican al considerar otra franja etaria en la que se mide la inversión pública en el capital humano de los hijos. 


\section{Referencias}

Ali, H. (2011). "Military expenditures and human development: guns and butter arguments revisited: a case study from Egypt”, Peace Economics, Peace Science and public policy, 17(1), 1554-8597.

Atkinson, A. y Bourguignon, F. (2000). "Income distribution and economics", en A. Atkinson y F. Bourguignon, eds., Handbook of Income Distribution. Amsterdam: Elsevier Science, 1-58.

Atlas, C. M, Gilligan, T. W., Hendershott, R. J. y Zupan, M. A. (1995). "Slicing the federal government net spending pie: who wins, loses and why", American Economic Review, 85(3), 624-629.

Becker, G. S. (1987). Tratado sobre la familia. Madrid: Alianza Editorial.

Becker, G. S. y Tomes, N. (1979). “An equilibrium theory of the distribution of income and intergenerational mobility", Journal of Political Economy, 87(6), 1153-89.

Behrman, J. R, Gaviria, A. y Székeley, M. (2001). "Intergenerational mobility in Latin America", Economia, 2(1), 1-31.

Behrman, J. R. y Taubman, P. (1990). "The intergenerational correlation between children's adult earnings and their parents' income: Results from the Michigan Panel Survey of Income Dynamics, Review of Income and Wealth, 36(2), 115-27.

Benoit, E. (1978). "Growth and defense in developing countries", Economic Development and Cultural Change, 26 (2), 271-280.

Bernal, R. y Camacho, A. (2010). "La importancia de los programas para la Primera Infancia en Colombia". Documento de trabajo No. 20. Universidad de los Andes.

Bhattacharya, D. y Mazumder, B. (2011). "A nonparametric analysis of black-white differences in intergenerational income mobility in the United States", Quantitative Economics, 2(3), 335-379.

Biswas, B., y R. Ram. (1986). "Military expenditures and economic growth in less developed countries: an augmented model and further evidence", Economic Development and Cultural Change, 34(2), 361-372.

Black, E. S. y Deveraux, P. J. (2011). "Recent developments in intergenerational mobility", En Ashenfelter, O. y Card, D., eds., Handbook of Labor Economics. Amsterdam: North-Holland Press. 
Boex, J. y Martinez-Vázquez, J. (2005). "The determinants of the incidence of intergovernmental grants: A survey of the international experience”, Andrew Young School of Policy Studies Working paper No. 09.

Brunello, G. y Checchi, D. (2006). "Does school tracking affect equality of opportunity? New international evidence", Institute for the Study of Labor, IZA Discussion Paper No. 2348.

Carneiro, P. y Heckman, J. (2003). "Human Capital Policy", The National Bureau of Economic Research, NBER Working Papers No. 9495.

Cetrángolo, O. y Gatto, F. (2002). "Descentralización Fiscal en Argentina: Restricciones Impuestas por un Proceso Mal Orientado", Documento presentado en el seminario Desarrollo Local y Regional: Hacia la construcción de Territorios Competitivos e Innovadores, Organizado por el ILPES y el Gobierno de la Provincia de Pichincha.

Corcoran, M., Gordon, R., Laren, D. y Gary, S. (1992). "The Association between men's economic status and their family and community origins," Journal of Human Resources, 27(3), 575-601.

Couch, K. A. y Lillard, D. R. (1998). "Sample selection rules and the intergenerational correlation of earnings", Labour Economics, 5(3), 313-29.

Cruces, G. (2008). “Tópicos de Economía distributiva”, Maestría en Economía, Facultad de Ciencias Económicas, Universidad Nacional de La Plata, La Plata, Material de clase no publicado.

Curcio, J., Cetrángolo, O. y Jiménez, J. (2009). "Fuentes de financiamiento educativo". En: Jiménez, Murdochowicz y de la educación que favorecen la cohesión social, CIEF-ADETEF.

DAGPyPS (2009). Gasto Público Social dirigido a la Niñez en la Argentina 1995-2007, Dirección de Análisis del Gasto Púbico y Programas Sociales (DAGPyPS) y Fondo de Naciones Unidas para Infancia (UNICEF), Buenos Aires.

DAGPyPS (2011). "Serie de gasto público consolidado. Aspectos metodológicos", Dirección de Análisis del Gasto Púbico y Programas Sociales (DAGPyPS).

Dakurah, H., S. Davies y R. Sampath. (2001). "Defense spending and economic growth in developing countries: A causality analysis", Journal of Policy Modeling, 23(6), 651-658. 
Davies, J., Zhang, J. y Zeng, J. (2005), "Intergenerational mobility under private vs. public education", Scandinavian Journal of Economics, 107(3), 399-417.

Deng, Z. y Treiman, D. J. (1997). "The impact of the cultural revolution on trends in educational attainment in the people's republic of China". The American Journal of Sociology, 103(2), 391-428.

Duflo, E. (2001). Schooling and labor market consequences of school construction in Indonesia: Evidence from an unusual policy experiment. The American Economic Review, 91(4), 795-813.

Duncan, G., Yeung, W. J., Brooks-Gunn, J. y Smith, J. R. (1998). "How much does childhood poverty affect the life chances of children?", American Sociological Review, 63(3), 406-423.

Duncan, G.J. y Brooks-Gunn, J. (1997). Consequences of growing up poor. New York: Russell Sage.

Fernández, A. G. (2006). "Alternative measures of intergeneracional social mobility in Argentina", Anales de la Asociación Argentina de Economía Política (AAEP), Buenos Aires.

Ferreres, O. J. (2010). Dos siglos de economía argentina 1810-2010: historia argentina en cifras. Fundación Norte y Sur; El Ateneo: Buenos Aires.

FIEL (2008). La igualdad de oportunidades en la Argentina. Movilidad intergeneracional en los 2000, Buenos Aires: Temas grupo editorial.

Fondo Monetario Internacional (2001), Manual de estadísticas de finanzas públicas. Washington DC: International Monetary Fund.

Gasparini, L. (2007). "Monitoring the socio-economic conditions in Argentina 19922006", CEDLAS, Centro de Estudios Distributivos, Laborales y Sociales, Facultad de Ciencias Económicas, Universidad Nacional de La Plata.

Gasparini, L., Alaimo, V, Cuenin, F., Rabassa, M. y Vúletin, G. (2000). "El impacto distributivo del gasto público en sectores sociales en la Provincia de Buenos Aires. Un análisis en base a la Encuesta de Desarrollo Social", Cuadernos de Economía $\mathrm{N}^{\circ}$ 50, Ministerio de Economía de la Provincia de Buenos Aires.

Gasparini, L., Jaume, D., Serio, M. y Vazquez, E. (2012). “La segregación escolar entre escuelas públicas y privadas en Argentina. Reconstruyendo la evidencia", Desarrollo Económico-Revista de Ciencias Sociales, 51, 189-219. 
Haider, S. y Solon, G. (2006). "Life-cycle variation in the association between current and lifetime earnings", The American Economic Review, 96(4), 1308-20.

Harknett, K., Garfinkel, I., Bainbridge, J., Smeeding, T., Folbre, N. y McLanahan, S. (2003). "Do public expenditures improve child outcomes in the U.S.: a comparison across fifty states", Center for Research on Child Wellbeing Working Paper No. 02.

Heckman, J. (2000). "Policies to foster human capital", Research in Economic, 54(1), 356.

Heckman. J. (2008). “Schools, Skills and Synapses”, Economic Inquiry, 46(3), 289-324.

Heckman, J. (1979), "Sample selection as a specification error", Econometrica, 47(1), 153-161.

Hertz, T., Meurs, M. y Selckuk, S. (2009). "Post-socialism: evidence from the Bulgarian case", World development, 37(3), 739-752.

Hertz, T., y Jayasundera, T. (2007). "School construction and intergenerational educational mobility in Indonesia", Department of Economics, American University, Washington, Working Paper No. 18.

Ichino, A., Karabarbounis, L. y Moretti, E. (2011), "The political economy of intergenerational income mobility", Economic Inquiry, 49(1), 47-69.

Jaume, D. J. (2013). "Un estudio sobre el incremento de la segregación escolar en Argentina", Documento de Trabajo $N^{\circ}$ 143, Centro de Estudios Distributivos, Laborales y Sociales (CEDLAS), Universidad Nacional de La Plata.

Jiménez, M. (2002). "Delimitación del perímetro de la consolidación contable pública y métodos de consolidación: especial referencia a las corporaciones locales", Revista de Contabilidad, 5(9), 147-167.

Kim, H. (1996). "Trade-offs between military spending, quality of life and economic growth", Comparative Economic Studies, 38(4), 69-84.

Knight, M., N. Loayzan, y Villanueva, D. (1996). “The peace dividend: military expenditure cuts and economic growth”, Policy Research Working Paper No. 1577.

Lillard, L. A., y Willis, R. (1994). "Intergenerational educational mobility: Effects of family and state in Malaysia", Journal of Human Resources, 29(4), 1126-1166.

Lillard, L. y Kilburn, M. (1995). “Intergenerational earnings links: sons and daughters", Corporation Provides Objective Research Service, RAND Working Paper Series No. 17. 
Machin, S. (2007), "Education expansion and intergenerational mobility in Britain", en L. Woessman y P. Peterson, eds., Schools and the equal opportunity problem, MIT Press.

Mayer, S. E. y Lopoo, L. M. (2008). "Government spending and intergeneracional mobility”, Journal of Public Ecoomics, 92(1-2), 139-158.

Mayer, S.E. (2002). "The influence of parental income on children's outcomes: a review report to the New Zealand", Ministry of Social Development.

Mazumder, B. (2005). "Fortunate sons: new estimates of intergenerational mobility in the United States using social security earnings data", The Review of Economics and Statistics, 87(2), 235-255.

Müller, A. y Raco, S. (2010). "Impacto fiscal de las transacciones del gobierno federal. Una evaluación para 2003-2008", Centro de Estudios de la Situación y Perspectivas de la Argentina, Universidad de Buenos Aires, Documento de trabajo No. 15.

Mulligan, C. (1997). Parental priorities and economic inequality. Chicago: University of Chicago Press.

Mulligan, C. (1999). "Galton vs. the human capital approach to inhertance: Some evidence", Journal of Political Economy, 107(6), Part. 2: Symposium on the Economic analysis of social behavior in honor of Gary S. Becker (Dec., 1999), S184S224.

Navarro, A. I. (2010). "Exploring intergenerational educational mobility in Argentina", en Bishop, J. A., ed., Studies in applied welfare analysis: Papers from the third ECINEQ Meeting (Vol. 18). Bingley: Emerald Group Publishing.

Nicoletti, C. (2008). "Multiple sample selection in the estimation of intergenerational occupational mobility", Institute for social \& Economic Research (ISER). University of Essex, Working paper No. 20.

Nicoletti, C. y Francesconi, M. (2006). "Intergenerational mobility and sample selection in short panels", Journal of Applied Econometrics, 21(8), 1265-93.

Núñez, J. y Miranda, L. (2007). "Recent findings on intergenerational income and educational mobility in Chile", Departamento de Economía, Universidad de Chile.

Núñez, J. y Risco, C. (2004). "Movilidad intergeneracional de ingresos en un país en desarrollo: El caso de Chile", Departamento de Economía, Universidad de Chile, Working Paper No. 210. 
Paqueo, V. y Lee, K. (2000). "Protecting the poor and improving investment in their human Capital", World Bank.

Payne, J. y Ross, K. (1992). "Defense spending and the macroeconomy", Defense Economics, 3(2), 161-168.

Pekkarinen, T.; Uusitalo, R. y Kerr, S. (2009), "School tracking and intergenerational income mobility: Evidence from the Finnish comprehensive school reform", Journal of Public Economics, 93(7-8), 965-973.

Porto, A. y Sanguinetti, P. (2001). "Political determinants of intergovernmental grants: Evidence from Argentina", Economics and Politics, 13(3), 237-253.

Quartulli, D. y Salvia, A. (2012). "La movilidad y la estratificación socio-ocupacional en la Argentina. Un análisis de las desigualdades de origen", Entramado y perspectivas, Revista de la carrera de Sociología, 2(2), 15-42.

Russett, M. (1969). “Who pays for defense?”, American Political Science Review, 63(2), 412-426.

Sánchez Hugalde, A. (2004). "Movilidad intergeneracional de ingresos y educativa en España (1980-90)”, Instituto de Economía de Barcelona, Document de treball No. 1.

Sanguinetti, J. y M., Tommasi. (2001). "Una propuesta de reforma integral del sistema fiscal federal", Centro de Estudios para el Desarrollo Institucional. Mimeo.

Sendón, M. A. (2005). "Las trayectorias de los egresados de la escuela media en una sociedad mutada", Revista Mexicana de Investigación Educativa, 10(24), 191-219.

Solon, G. (1992). "Intergenerational income mobility in the United States", The American Economic Review, 82(3), 393-408.

Solon, G. (2004). "A model of intergenerational mobility variation over time and place", en M. Corak, ed., Generational Income Mobility in North America and Europe. Cambridge: Cambridge University Press.

Yildirim, J. y Sezgin, S. (2002). "Defense, education and health expenditures in Turkey", Journal of Peace Research, 39(5), 569-580.

Zapata, J. A., Bertea, A. O. y Iturre, T. B. (2000). "Sistema de supervisión multilateral: para un federalismo con responsabilidad fiscal", Reunión anual 2000 de ABA y Expobank, Tucumán. 


\section{Anexo}

Cuadro A1. Edades de los hijos de la muestra principal según año y cohorte de nacimiento

\begin{tabular}{|c|c|c|c|c|c|c|c|c|c|c|c|c|c|c|c|c|c|c|c|c|}
\hline \multirow{3}{*}{$\begin{array}{l}\text { Cohorte de } \\
\text { nacimiento }\end{array}$} & & & & & & \multicolumn{15}{|c|}{ Edades en cada año } \\
\hline & & & & & & & & & & & 1 & 2 & 3 & 4 & 5 & 6 & 7 & 8 & 9 & 10 \\
\hline & $\overline{1993}$ & 1994 & 1995 & 1996 & 1997 & 1998 & 1999 & 2000 & 2001 & 2002 & 2003 & 2004 & 2005 & 2006 & 2007 & 2008 & 2009 & 2010 & 2011 & 2012 \\
\hline 11978 & 15 & 16 & 17 & 18 & 19 & 20 & 21 & 22 & 23 & 24 & 25 & 26 & 27 & 28 & 29 & 30 & 31 & 32 & 33 & 34 \\
\hline 21979 & & 15 & 16 & 17 & 18 & 19 & 20 & 21 & 22 & 23 & 24 & 25 & 26 & 27 & 28 & 29 & 30 & 31 & 32 & 33 \\
\hline 31980 & & & 15 & 16 & 17 & 18 & 19 & 20 & 21 & 22 & 23 & 24 & 25 & 26 & 27 & 28 & 29 & 30 & 31 & 32 \\
\hline $4 \quad 1981$ & & & & 15 & 16 & 17 & 18 & 19 & 20 & 21 & 22 & 23 & 24 & 2 & 26 & 27 & 28 & 29 & 30 & 31 \\
\hline $5 \quad 1982$ & & & & & 15 & 16 & 17 & 18 & 19 & 20 & 21 & 22 & 23 & 24 & 25 & 26 & 27 & 28 & 29 & 30 \\
\hline $\begin{array}{ll}6 & 1983\end{array}$ & & & & & & 15 & 16 & 17 & 18 & 1 & 20 & 2 & 22 & 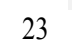 & 24 & 25 & 26 & 27 & 28 & 29 \\
\hline $7 \quad 1984$ & & & & & & & 15 & 16 & 17 & 18 & 19 & 20 & 21 & 22 & 23 & 24 & 25 & 26 & 27 & 28 \\
\hline $8 \quad 1985$ & & & & & & & & 15 & 16 & 17 & 18 & 19 & 20 & 21 & 22 & 23 & 24 & 25 & 26 & 27 \\
\hline $9 \quad 1986$ & & & & & & & & & 15 & 16 & 17 & 18 & 19 & 20 & 21 & 22 & 23 & 24 & 25 & 26 \\
\hline $10 \quad 1987$ & & & & & & & & & & 15 & 16 & 17 & 18 & 19 & 20 & 21 & 22 & 23 & 24 & 25 \\
\hline
\end{tabular}

Fuente: Elaboración propia.

Nota: En blanco se señalan las edades en las que se mide la inversión pública en capital humano utilizando los datos del gasto público consolidado; en gris oscuro, las edades en las que se mide esa inversión a partir de la serie del gasto público provincial disponibles para el período 1993-2009 y en gris claro, se indican las edades de los hijos de la muestra principal. 
Cuadro A2. Brechas en la movilidad intergeneracional educativa según nivel de gasto público

\begin{tabular}{|c|c|c|c|c|c|c|c|c|c|c|}
\hline \multirow{2}{*}{\begin{tabular}{|c|} 
Medida \\
de GPCpe
\end{tabular}} & \multirow{2}{*}{ Variables } & \multicolumn{3}{|c|}{ Nivel de gasto bajo } & \multicolumn{3}{|c|}{ Nivel de gasto medio } & \multicolumn{3}{|c|}{ Nivel de gasto alto } \\
\hline & & Modelo 1 & Modelo 2 & odelo 3 & Modelo 1 & Modelo 2 & Modelo 3 & Modelo 1 & Modelo 2 & Modelo3 \\
\hline \multirow[t]{6}{*}{ Total } & Educación del padre & & & & $0.209 * * *$ & $0.209^{* * *}$ & $0.209 * * *$ & $0.137 * * *$ & $0.137^{* * * *}$ & $0.142 * * *$ \\
\hline & & $(0.157-0.275)$ & $(0.161-0.277)$ & $(0.157-0.291)$ & $(0.160-0.258)$ & $(0.159-0.258)$ & $(0.152-0.266)$ & $(0.088-0.185)$ & $(0.080-0.194)$ & $0.084-0.201)$ \\
\hline & Educe & & & & & & & & & \\
\hline & & $(0.07)$ & $(0.0$ & $(0.06$ & $(0.025-0.209)$ & $(0.02$ & $.220)$ & $(0.075-0.223)$ & $(0.070-0.229)$ & $(0.066-0.224)$ \\
\hline & Nivele & $-3.994 * * *$ & $-4.038^{* * *}$ & -4.0 & $-4.142 * * *$ & $-4.134 * * *$ & $-4.125^{* * *}$ & $-2.838^{* * *}$ & $-2.885^{* * *}$ & $-2.971 * * *$ \\
\hline & & $(-4.831--3.157)$ & $(-4.863--3.213)$ & $(-5.010--3.183)$ & $(-5.083--3.201)$ & $(-5.106--3.161)$ & $(-5.228--3.021)$ & $(-3.475--2.202)$ & $(-3.636--2.134)$ & $(-3.727--2.215)$ \\
\hline \multirow{6}{*}{$\begin{array}{l}\text { Social } \\
\text { total }\end{array}$} & & & & & & & & $0^{* * *}$ & & \\
\hline & & 260) & $(0.1$ & $(0.1$ & 273) & 278) & 278) & $0.201)$ & 211) & 214) \\
\hline & Educa & $5 * * *$ & & & & & & & & \\
\hline & & $0.233)$ & $(0.10$ & $(0.09$ & $0.184)$ & $-0.189)$ & $(0.03$ & $0.167)$ & $(0.0$ & $0.163)$ \\
\hline & Niv & & & & & & & & & \\
\hline & & $(-4.80$ & $(-4.78$ & $(-4.88$ & $(-4.88$ & $(-4.98$ & $-3.243)$ & $-2.824)$ & $-2.774)$ & 2.851) \\
\hline \multirow[t]{6}{*}{ GPSCH1 } & & & & & *** & 0 & & & & \\
\hline & & $(0.1$ & 0.1 & 30) & $(0.1$ & & & $(0.1$ & & \\
\hline & $E d u c$ & & $* *$ & & & & & & & \\
\hline & & $(0.09$ & $(0.0$ & $(0.08$ & $(0.01$ & $0.227)$ & $(0.00$ & $0.187)$ & & 187) \\
\hline & Nive & -3 & & & & -4 & & -3 & -3 & \\
\hline & & $(-4.6$ & $(-4.7$ & $(-4.7$ & $(-5.3$ & $(-5.4$ & 11) & $(-3.4$ & .77) & 571) \\
\hline \multirow[t]{6}{*}{ GPSCH2 } & Educc & $4^{* * * *}$ & $0.215^{* * *}$ & $0.216^{* * *}$ & $0.191^{* * *}$ & $0.189^{* * *}$ & $0.194 * * *$ & $0.151^{* * *}$ & $0.149^{* * *}$ & $0.155^{* * *}$ \\
\hline & & $0.277)$ & $.275)$ & 282) & $(0.11)$ & $(0.1$ & .276) & $0.193)$ & & 206) \\
\hline & Educac & & 0 & & & & & & & \\
\hline & & $(0.0$ & $(0.0$ & $(0.0$ & $(0.02$ & $(0.0$ & 70) & $(0.0$ & 97) & 189) \\
\hline & Nivel ed. ma & $-3.970 * * *$ & $-3.987 * * *$ & $-3.997 * * *$ & $-3.873 * * *$ & $-3.845 * * *$ & $-3.892 * * *$ & $-3.107 * * *$ & $-3.125 * * *$ & $-3.220 * * *$ \\
\hline & & 4.86 & $(-4.8$ & $(-4.9$ & $(-5.18)$ & $(-5.2)$ & $(-5.3$ & $(-3.5$ & $(-3.6)-(-) \cdot-)$ & $(-3.7$ \\
\hline \multirow[t]{6}{*}{ GPSCH3 } & Educc & $4 * * *$ & **** & *** & $0^{* * * *}$ & $0^{* * *}$ & $3 * * *$ & $7 * * *$ & $5 * * *$ & $0 * * *$ \\
\hline & & $0.276)$ & $(0.1$ & $(0.15$ & $-0.255)$ & $(0.12$ & $(0.12)$ & $0.193)$ & 200) & $(0.093$ \\
\hline & Educación del padre & $0.136^{* * *}$ & $0.134 * * *$ & $0.129^{* * *}$ & $0.142 * * *$ & $0.140^{* * *}$ & $0.139 * *$ & $0.130^{* * *}$ & $0.131^{* * * *}$ & 0.1 \\
\hline & & $(0.084-0.18$ & $(0.08$ & $(0.07$ & $(0.04$ & $-0.240)$ & $(0.02$ & $(0.0$ & 198) & $0.194)$ \\
\hline & Nivel & $-3.944 * * *$ & $-3.974 * * *$ & -4.0 & $-3.824 * * *$ & -3 . & -3 & -3 & -3 & -3 . \\
\hline & & 4.8 & & $(-4.9$ & $(-50)$ & & & $(-3.5)$ & & 563) \\
\hline \multirow[t]{6}{*}{$\overline{G P S C H 4}$} & Educación del padre $\times$ Nivel & $0.188 * * *$ & $0.191 * * *$ & $0.194 * * *$ & $0.203 * * *$ & $0.202 * * *$ & $0.201 * * *$ & $0.156^{* * *}$ & $0.155^{* * *}$ & $0.159 * * *$ \\
\hline & er & $(0.113-0.263)$ & $(0.118-0.264)$ & $(0.114-0.273)$ & $(0.140-0.267)$ & $0.267)$ & $(0.136$ & $(0.107-0.204)$ & $(0.098-0.212)$ & $0.217)$ \\
\hline & Educación del padre & & $0.178^{* * *}$ & & & & & & & \\
\hline & & 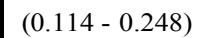 & 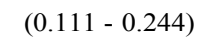 & م & $(0.01 \xi$ & $(0.0$ & $(0.0$ & $(0.06$ & $(0.05$ & $(0.05$ \\
\hline & Nivel ed. madre bajo & $-3.398^{* * *}$ & $-3.440^{* * *}$ & $-3.439 * * *$ & $-4.148 * * *$ & $-4.129 * * *$ & $-4.129 * * *$ & $-3.235^{* * *}$ & $-3.260^{* * *}$ & $-3.323^{* * *}$ \\
\hline & & $(-4.499--2.296)$ & $(-4.530--2.350)$ & $(-4.624--2.255)$ & $(-5.435--2.861)$ & $(-5.457--2.801)$ & $(-5.461--2.797)$ & $(-3.712--2.759)$ & $(-3.841--2.679)$ & $(-3.887--2.759)$ \\
\hline
\end{tabular}

Fuente: Elaboración propia sobre la base de varias fuentes de información. Ver la sección de datos para más detalles.

Nota: *** $p$-value $<0.01$, ** $p$-value $<0.05, *$-value $<0.1$. Entre paréntesis se reportan los intervalos de confianza al $95 \%$ computados a partir de errores estándares agrupados a nivel provincial. Ver Cuadro 3 con la descripción de la especificación de cada modelo. 


\section{Cuadro A3. Diferencias en diferencias de la EII por nivel educativo del padre según nivel de gasto público}

\begin{tabular}{|c|c|c|c|c|c|c|c|c|c|}
\hline \multirow{3}{*}{$\begin{array}{c}\text { Medidas de gasto } \\
\text { público consolidado } \\
\text { per cápita }\end{array}$} & \multicolumn{9}{|c|}{ Difference in difference EII } \\
\hline & \multicolumn{3}{|c|}{ Nivel de gasto bajo - medio } & \multicolumn{3}{|c|}{ Nivel de gasto medio - alto } & \multicolumn{3}{|c|}{ Nivel de gasto bajo-alto } \\
\hline & Mod. 1 & Mod. 2 & Mod. 3 & Mod. 1 & Mod. 2 & Mod. 3 & Mod. 1 & Mod. 2 & Mod. 3 \\
\hline Total & $\begin{array}{l}0.573 * * * \\
(0.000)\end{array}$ & $\begin{array}{cc}0.585 & * * * \\
(0.000) & \end{array}$ & $\begin{array}{cc}0.529 & * * * \\
(0.000) & \end{array}$ & $\begin{array}{l}0.365 \quad * * * \\
(0.000)\end{array}$ & $\begin{array}{c}0.374 \quad * * * \\
(0.000)\end{array}$ & $\begin{array}{c}0.394 \quad * * * * \\
(0.000)\end{array}$ & $\begin{array}{cc}0.939 \\
(0.000)\end{array}$ & $\begin{array}{c}0.959 \quad * * * \\
(0.000)\end{array}$ & $\begin{array}{l}0.923 \quad * * * \\
(0.000)\end{array}$ \\
\hline Social total & $\begin{array}{l}-0.091 \\
(0.521)\end{array}$ & $\begin{array}{l}-0.074 \\
(0.570)\end{array}$ & $\begin{array}{l}-0.109 \\
(0.456)\end{array}$ & $\begin{array}{c}0.298 \\
(0.284)\end{array}$ & $\begin{array}{c}0.263 \\
(0.394)\end{array}$ & $\begin{array}{c}0.291 \\
(0.339)\end{array}$ & $\begin{array}{c}0.208 \\
(0.185)\end{array}$ & $\begin{array}{c}0.188 \\
(0.226)\end{array}$ & $\begin{array}{c}0.182 \\
(0.252)\end{array}$ \\
\hline GPSCHN1 & $\begin{array}{l}-0.025 \\
(0.836)\end{array}$ & $\begin{array}{l}-0.020 \\
(0.841)\end{array}$ & $\begin{array}{l}-0.063 \\
(0.713)\end{array}$ & $\begin{array}{l}0.664 \quad * * * \\
(0.000)\end{array}$ & $\begin{array}{c}0.646 \quad * * * \\
(0.000)\end{array}$ & $\begin{array}{c}0.711 \quad * * * \\
(0.000)\end{array}$ & $\begin{array}{c}0.638 \quad * * * \\
(0.000)\end{array}$ & $\begin{array}{c}0.626 \quad * * * \\
(0.001)\end{array}$ & $\begin{array}{l}0.648 \quad * * * \\
(0.000)\end{array}$ \\
\hline GPSCHN2 & $\begin{array}{l}-0.085 \\
(0.649)\end{array}$ & $\begin{array}{l}-0.098 \\
(0.611)\end{array}$ & $\begin{array}{l}-0.109 \\
(0.584)\end{array}$ & $\begin{array}{l}0.688 \quad * * * \\
(0.000)\end{array}$ & $\begin{array}{c}0.688 \quad * * * \\
(0.000)\end{array}$ & $\begin{array}{l}0.701 \quad * * * \\
(0.000)\end{array}$ & $\begin{array}{c}0.603 \quad * * * \\
(0.002)\end{array}$ & $\begin{array}{c}0.590 \quad * * * \\
(0.003)\end{array}$ & $\begin{array}{l}0.592 \quad * * * \\
(0.004)\end{array}$ \\
\hline GPSCHN3 & $\begin{array}{l}-0.023 \\
(0.832)\end{array}$ & $\begin{array}{l}-0.016 \\
(0.841)\end{array}$ & $\begin{array}{l}-0.053 \\
(0.733)\end{array}$ & $\begin{array}{l}0.777 \quad * * * \\
(0.000)\end{array}$ & $\begin{array}{c}0.758 \quad * * * \\
(0.000)\end{array}$ & $\begin{array}{c}0.784 \quad * * * \\
(0.000)\end{array}$ & $\begin{array}{c}0.754 \quad * * * \\
(0.000)\end{array}$ & $\begin{array}{c}0.742 \quad * * * \\
(0.000)\end{array}$ & $\begin{array}{l}0.731 \quad * * * \\
(0.000)\end{array}$ \\
\hline GPSCHN4 & $\begin{array}{l}-0.131 \\
(0.426)\end{array}$ & $\begin{array}{l}-0.126 \\
(0.444)\end{array}$ & $\begin{array}{l}-0.167 \\
(0.334)\end{array}$ & $\begin{array}{c}0.636 \quad * * * \\
(0.000)\end{array}$ & $\begin{array}{c}0.619 \quad * * * \\
(0.000)\end{array}$ & $\begin{array}{c}0.673 \quad * * * * \\
(0.000)\end{array}$ & $\begin{array}{c}0.505 \\
(0.002)\end{array} \quad * * * *$ & $\begin{array}{c}0.494 \quad * * * \\
(0.003)\end{array}$ & $\begin{array}{l}0.507 \quad * * * \\
(0.004)\end{array}$ \\
\hline GPSCHN5 & $\begin{array}{l}-0.075 \\
(0.531)\end{array}$ & $\begin{array}{l}-0.075 \\
(0.527) \\
\end{array}$ & $\begin{array}{l}-0.075 \\
(0.501) \\
\end{array}$ & $\begin{array}{r}0.293 \\
(0.264) \\
\end{array}$ & $\begin{array}{c}0.287 \\
(0.280) \\
\end{array}$ & $\begin{array}{c}0.202 \\
(0.467) \\
\end{array}$ & $\begin{array}{c}0.217 \\
(0.100)\end{array}$ & $\begin{array}{c}0.213 \\
(0.102) \\
\end{array}$ & $\begin{array}{r}0.128 \\
(0.294) \\
\end{array}$ \\
\hline
\end{tabular}

Fuente: Elaboración propia sobre la base de varias fuentes de información. Ver la sección de datos para más detalles.

Nota: los niveles de gasto bajo, medio y alto corresponden al primer, quinto y último decil de las distribuciones de cada medida de gasto público consideradas, respectivamente.

Cuadro A4. Efectos del gasto público sobre la probabilidad de los hijos superar el decil en que se encontraba el padre. Modelo probit $(\tau=0.10)$.

\begin{tabular}{|c|c|c|c|c|c|c|c|c|c|}
\hline \multirow{2}{*}{$\begin{array}{c}\text { Medidas de gasto } \\
\text { público consolidado per }\end{array}$} & \multicolumn{2}{|c|}{ "Modelo 1} & \multicolumn{3}{|c|}{ Modelo 2} & \multicolumn{4}{|c|}{ Modelo 3} \\
\hline & Coef & Ef. Marg. & Coe & & Ef. Marg. & Coef & & Ef. Mar & \\
\hline Total & $\begin{array}{c}0.020 \\
(0.127)\end{array}$ & $\begin{array}{l}0.0001 \\
(0.050)\end{array}$ & $\begin{array}{c}1.182 \\
(0.600)\end{array}$ & $* *$ & $\begin{array}{ll}0.005 & * * \\
(0.236) & \end{array}$ & $\begin{array}{c}1.403 \\
(0.793)\end{array}$ & & $\begin{array}{c}0.006 \\
(0.312)\end{array}$ & $*$ \\
\hline Social total & $\begin{array}{c}0.037 \\
(0.105)\end{array}$ & $\begin{array}{l}0.0001 \\
(0.041)\end{array}$ & $\begin{array}{c}1.557 \\
(0.750)\end{array}$ & $* *$ & $\begin{array}{l}0.006 * * \\
(0.295)\end{array}$ & $\begin{array}{c}1.841 \\
(0.910)\end{array}$ & $* *$ & $\begin{array}{c}0.007 \\
(0.358)\end{array}$ & $* *$ \\
\hline GPSCH1 & $\begin{array}{l}-0.002 \\
(0.107)\end{array}$ & $\begin{array}{l}0.0000 \\
(0.042)\end{array}$ & $\begin{array}{c}0.786 \\
(0.689)\end{array}$ & & $\begin{array}{c}0.003 \\
(0.272)\end{array}$ & $\begin{array}{c}1.192 \\
(0.771)\end{array}$ & & $\begin{array}{c}0.005 \\
(0.304)\end{array}$ & \\
\hline GPSCH2 & $\begin{array}{c}0.005 \\
(0.114)\end{array}$ & $\begin{array}{l}0.0000 \\
(0.045)\end{array}$ & $\begin{array}{c}0.888 \\
(0.600)\end{array}$ & & $\begin{array}{c}0.004 \\
(0.236)\end{array}$ & $\begin{array}{c}1.379 \\
(0.790)\end{array}$ & $*$ & $\begin{array}{c}0.005 \\
(0.311)\end{array}$ & $*$ \\
\hline GPSCH3 & $\begin{array}{c}0.017 \\
(0.118)\end{array}$ & $\begin{array}{l}0.0001 \\
(0.047)\end{array}$ & $\begin{array}{c}1.023 \\
(0.759)\end{array}$ & & $\begin{array}{c}0.004 \\
(0.299)\end{array}$ & $\begin{array}{c}1.705 \\
(0.867)\end{array}$ & $* *$ & $\begin{array}{c}0.007 \\
(0.341)\end{array}$ & $* *$ \\
\hline GPSCH4 & $\begin{array}{c}0.022 \\
(0.125)\end{array}$ & $\begin{array}{l}0.0001 \\
(0.049)\end{array}$ & $\begin{array}{c}1.488 \\
(0.671)\end{array}$ & $* *$ & $\begin{array}{l}0.006 * * \\
(0.264)\end{array}$ & $\begin{array}{c}1.876 \\
(0.829)\end{array}$ & $* *$ & $\begin{array}{c}0.007 \\
(0.326)\end{array}$ & $* *$ \\
\hline GPSCH5 & $\begin{array}{c}0.031 \\
(0.129)\end{array}$ & $\begin{array}{l}0.0001 \\
(0.051) \\
\end{array}$ & $\begin{array}{c}1.335 \\
(0.793)\end{array}$ & $*$ & $\begin{array}{c}0.005 * \\
(0.312)\end{array}$ & $\begin{array}{c}1.584 \\
(0.949) \\
\end{array}$ & $*$ & $\begin{array}{c}0.006 \\
(0.373) \\
\end{array}$ & $*$ \\
\hline
\end{tabular}

Fuente: Elaboración propia sobre la base de varias fuentes de información. Ver la sección de datos para más detalles.

Nota: $* * * p$-value $<0.01, * * p$-value $<0.05, * p$-value $<0.1$. Entre paréntesis se reportan los errores estándares agrupados a nivel provincial. Ver la nota del Cuadro 3 con la descripción de la especificación de cada modelo. Los efectos marginales fueron computados en los valores promedios de las variables explicativas. 


\section{Cuadro A5. Efecto de las categorías del gasto público social provincial per cápita}

sobre la elasticidad intergeneracional del ingreso

\begin{tabular}{|c|c|c|c|c|}
\hline $\begin{array}{l}\text { Categorías } \\
\text { GPP } \\
\end{array}$ & Variables de interés & Mod. 1 & Mod. 2 & Mod. 3 \\
\hline Salud & $\begin{array}{l}\text { Log del Ingreso del padre } \\
\text { Log Gasto público per cápita } \\
\text { Log del Ingreso del padre } \times \text { Log Gasto público per } \\
\text { cápita }\end{array}$ & $\begin{array}{c}0.721 * * * \\
(0.094) \\
0.108 \\
(0.133) \\
-0.017 \\
(0.019) \\
\end{array}$ & $\begin{array}{c}0.798 * * * \\
(0.091) \\
0.104 \\
(0.105) \\
-0.033^{*} \\
(0.018) \\
\end{array}$ & $\begin{array}{c}0.863^{* * *} \\
(0.097) \\
0.144 \\
(0.104) \\
-0.046^{* *} \\
(0.020) \\
\end{array}$ \\
\hline $\begin{array}{l}\text { Atención } \\
\text { médica }\end{array}$ & $\begin{array}{l}\text { Log del Ingreso del padre } \\
\text { Log Gasto público per cápita } \\
\text { Log del Ingreso del padre } \times \text { Log Gasto público per } \\
\text { cápita }\end{array}$ & $\begin{array}{c}0.679 * * * \\
(0.078) \\
0.018 \\
(0.107) \\
-0.009 \\
(0.017)\end{array}$ & $\begin{array}{c}0.731 * * * \\
(0.077) \\
0.068 \\
(0.083) \\
-0.021 \\
(0.016)\end{array}$ & $\begin{array}{c}0.789 * * * \\
(0.078) \\
0.129 \\
(0.089) \\
-0.034 * \\
(0.017)\end{array}$ \\
\hline Obras sociales & $\begin{array}{l}\text { Log del Ingreso del padre } \\
\text { Log Gasto público per cápita } \\
\text { Log del Ingreso del padre } \times \text { Log Gasto público per } \\
\text { cápita }\end{array}$ & $\begin{array}{c}0.731 * * * \\
(0.133) \\
0.299 \\
(0.188) \\
-0.024 \\
(0.035) \\
\end{array}$ & $\begin{array}{c}0.811^{* * *} \\
(0.130) \\
0.335^{*} \\
(0.163) \\
-0.045 \\
(0.035) \\
\end{array}$ & $\begin{array}{c}0.792 * * * \\
(0.133) \\
0.249 \\
(0.159) \\
-0.040 \\
(0.036) \\
\end{array}$ \\
\hline $\begin{array}{l}\text { Promoción y } \\
\text { Asistencia } \\
\text { Social }\end{array}$ & $\begin{array}{l}\text { Log del Ingreso del padre } \\
\text { Log Gasto público per cápita } \\
\begin{array}{l}\text { Log del Ingreso del padre } \times \text { Log Gasto público per } \\
\text { cápita }\end{array}\end{array}$ & $\begin{array}{c}1.036 * * * \\
(0.124) \\
0.519 * * * \\
(0.184) \\
-0.109 * * * \\
(0.034) \\
\end{array}$ & $\begin{array}{c}1.069 * * * \\
(0.122) \\
0.524 * * * \\
(0.149) \\
-0.118 * * * \\
(0.033)\end{array}$ & $\begin{array}{c}1.112 * * * \\
(0.134) \\
0.621 * * * \\
(0.167) \\
-0.130 * * * \\
(0.037) \\
\end{array}$ \\
\hline $\begin{array}{l}\text { Promoción y } \\
\text { asistencia } \\
\text { social pública }\end{array}$ & $\begin{array}{l}\text { Log del Ingreso del padre } \\
\text { Log Gasto público per cápita } \\
\text { Log del Ingreso del padre } \times \text { Log Gasto público per } \\
\text { cápita }\end{array}$ & $\begin{array}{c}1.052 * * * \\
(0.122) \\
0.543 * * * \\
(0.182) \\
-0.114 * * * \\
(0.034) \\
\end{array}$ & $\begin{array}{c}1.077 * * * \\
(0.120) \\
0.540 * * * \\
(0.150) \\
-0.121 * * * \\
(0.033) \\
\end{array}$ & $\begin{array}{c}1.114 * * * \\
(0.131) \\
0.635 * * * \\
(0.168) \\
-0.131 * * * \\
(0.037) \\
\end{array}$ \\
\hline $\begin{array}{l}\text { Educación y } \\
\text { Cultura }\end{array}$ & $\begin{array}{l}\text { Log del Ingreso del padre } \\
\text { Log Gasto público per cápita } \\
\begin{array}{l}\text { Log del Ingreso del padre } \times \text { Log Gasto público per } \\
\text { cápita }\end{array}\end{array}$ & $\begin{array}{c}1.240 * * * \\
(0.211) \\
0.655 * * * \\
(0.211) \\
-0.110 * * \\
(0.040) \\
\end{array}$ & $\begin{array}{c}1.328 * * * \\
(0.215) \\
0.608^{* * *} \\
(0.197) \\
-0.126^{* * *} \\
(0.041) \\
\end{array}$ & $\begin{array}{c}1.404 * * * \\
(0.248) \\
0.622 * * \\
(0.233) \\
-0.141 * * * \\
(0.047) \\
\end{array}$ \\
\hline $\begin{array}{l}\text { Educación } \\
\text { básica }\end{array}$ & $\begin{array}{l}\text { Log del Ingreso del padre } \\
\text { Log Gasto público per cápita } \\
\begin{array}{l}\text { Log del Ingreso del padre } \times \text { Log Gasto público per } \\
\text { cápita }\end{array}\end{array}$ & $\begin{array}{c}0.982 * * * \\
(0.235) \\
0.415 \\
(0.254) \\
-0.066 \\
(0.047) \\
\end{array}$ & $\begin{array}{c}1.069 * * * \\
(0.244) \\
0.371 \\
(0.241) \\
-0.082 \\
(0.049) \\
\end{array}$ & $\begin{array}{c}1.115^{* * *} \\
(0.264) \\
0.370 \\
(0.265) \\
-0.091^{*} \\
(0.053) \\
\end{array}$ \\
\hline $\begin{array}{l}\text { Educación } \\
\text { superior y } \\
\text { universitaria }\end{array}$ & $\begin{array}{l}\text { Log del Ingreso del padre } \\
\text { Log Gasto público per cápita } \\
\text { Log del Ingreso del padre } \times \text { Log Gasto público per } \\
\text { cápita }\end{array}$ & $\begin{array}{c}0.845^{* * *} \\
(0.085) \\
0.416^{* *} \\
(0.158) \\
-0.077 * * \\
(0.030) \\
\end{array}$ & $\begin{array}{c}0.843 * * * \\
(0.082) \\
0.403 * * \\
(0.157) \\
-0.076 * * \\
(0.029) \\
\end{array}$ & $\begin{array}{c}0.863 * * * \\
(0.082) \\
0.446^{* *} \\
(0.161) \\
-0.084 * * * \\
(0.029) \\
\end{array}$ \\
\hline $\begin{array}{l}\text { Vivienda y } \\
\text { Urbanis mo }\end{array}$ & $\begin{array}{l}\text { Log del Ingreso del padre } \\
\text { Log Gasto público per cápita } \\
\begin{array}{l}\text { Log del Ingreso del padre } \times \text { Log Gasto público per } \\
\text { cápita }\end{array}\end{array}$ & $\begin{array}{c}0.476 * * * \\
(0.060) \\
-0.207 \\
(0.125) \\
0.053 * * \\
(0.021) \\
\end{array}$ & $\begin{array}{c}0.508^{* * *} \\
(0.060) \\
-0.163 \\
(0.133) \\
0.043^{*} \\
(0.022) \\
\end{array}$ & $\begin{array}{c}0.503 * * * \\
(0.063) \\
-0.149 \\
(0.138) \\
0.044 * \\
(0.022) \\
\end{array}$ \\
\hline $\begin{array}{l}\text { Agua y } \\
\text { alcantarillado }\end{array}$ & $\begin{array}{l}\text { Log del Ingreso del padre } \\
\text { Log Gasto público per cápita } \\
\begin{array}{l}\text { Log del Ingreso del padre } \times \text { Log Gasto público per } \\
\text { cápita }\end{array}\end{array}$ & $\begin{array}{c}0.601 * * * \\
(0.059) \\
-0.113 \\
(0.144) \\
0.019 \\
(0.027)\end{array}$ & $\begin{array}{c}0.598 * * * \\
(0.059) \\
-0.105 \\
(0.140) \\
0.021 \\
(0.027)\end{array}$ & $\begin{array}{c}0.596^{* * *} \\
(0.061) \\
-0.097 \\
(0.144) \\
0.021 \\
(0.028)\end{array}$ \\
\hline
\end{tabular}

Fuente: Elaboración propia sobre la base de varias fuentes de información. Ver la sección de datos para más detalles.

Nota: $* * * p$-value $<0.01, * * p$-value $<0.05, * p$-value $<0.1$. Entre paréntesis se reportan los errores estándares agrupados a nivel provincial. Ver la nota del Cuadro 12 con la descripción de la especificación de cada modelo. 


\section{Cuadro A6. Efecto de las categorías del gasto público social provincial per cápita sobre el coeficiente de persistencia educativa intergeneracional}

\begin{tabular}{|c|c|c|c|c|}
\hline \begin{tabular}{|l|} 
Categorías \\
GPP
\end{tabular} & Variables de interés & Mod. 1 & Mod. 2 & Mod. 3 \\
\hline Salud & $\begin{array}{l}\text { Educación del padre } \\
\text { Log Gasto público per cápita } \\
\begin{array}{l}\text { Educación del padre } \times \text { Log Gasto público per } \\
\text { cápita }\end{array}\end{array}$ & $\begin{array}{c}0.758 * * * \\
(0.149) \\
0.908 * \\
(0.500) \\
-0.077 * * \\
(0.030)\end{array}$ & $\begin{array}{c}0.733 * * * \\
(0.148) \\
1.576^{* * *} \\
(0.537) \\
-0.072^{* *} \\
(0.030)\end{array}$ & $\begin{array}{c}0.732 * * * \\
(0.142) \\
1.230 * \\
(0.621) \\
-0.072 * * \\
(0.029)\end{array}$ \\
\hline $\begin{array}{l}\text { Atención } \\
\text { médica }\end{array}$ & $\begin{array}{l}\text { Educación del padre } \\
\text { Log Gasto público per cápita } \\
\begin{array}{l}\text { Educación del padre } \times \text { Log Gasto público per } \\
\text { cápita }\end{array}\end{array}$ & $\begin{array}{c}0.701 * * * \\
(0.087) \\
0.755^{* * *} \\
(0.266) \\
-0.072 * * * \\
(0.019) \\
\end{array}$ & $\begin{array}{c}0.681 * * * \\
(0.087) \\
1.200 * * * \\
(0.362) \\
-0.067 * * * \\
(0.019) \\
\end{array}$ & $\begin{array}{c}0.678 * * * \\
(0.082) \\
0.927 * \\
(0.456) \\
-0.067 * * * \\
(0.018)\end{array}$ \\
\hline Obras sociales & $\begin{array}{l}\text { Educación del padre } \\
\text { Log Gasto público per cápita } \\
\begin{array}{l}\text { Educación del padre } \times \text { Log Gasto público per } \\
\text { cápita }\end{array}\end{array}$ & $\begin{array}{c}0.400 * * * \\
(0.082) \\
0.549 \\
(0.538) \\
-0.005 \\
(0.022) \\
\end{array}$ & $\begin{array}{c}0.393 * * * \\
(0.081) \\
2.052 * * * \\
(0.518) \\
-0.003 \\
(0.022) \\
\end{array}$ & $\begin{array}{c}0.402 * * * \\
(0.084) \\
1.959 * * * \\
(0.582) \\
-0.006 \\
(0.023)\end{array}$ \\
\hline $\begin{array}{l}\text { Promoción y } \\
\text { Asistencia } \\
\text { Social }\end{array}$ & $\begin{array}{l}\text { Educación del padre } \\
\text { Log Gasto público per cápita } \\
\begin{array}{l}\text { Educación del padre } \times \text { Log Gasto público per } \\
\text { cápita }\end{array}\end{array}$ & $\begin{array}{c}0.735^{* * *} \\
(0.145) \\
0.891 * \\
(0.438) \\
-0.097 * * \\
(0.041) \\
\end{array}$ & $\begin{array}{c}0.709^{* * *} \\
(0.137) \\
0.970^{* *} \\
(0.405) \\
-0.090^{* *} \\
(0.039) \\
\end{array}$ & $\begin{array}{c}0.708 * * * \\
(0.135) \\
0.894 * \\
(0.490) \\
-0.090 * * \\
(0.038)\end{array}$ \\
\hline $\begin{array}{l}\text { Promoción y } \\
\text { asistencia } \\
\text { social pública }\end{array}$ & $\begin{array}{l}\text { Educación del padre } \\
\text { Log Gasto público per cápita } \\
\begin{array}{l}\text { Educación del padre } \times \text { Log Gasto público per } \\
\text { cápita }\end{array}\end{array}$ & $\begin{array}{c}0.740 * * * \\
(0.134) \\
0.899 * * \\
(0.410) \\
-0.099 * * \\
(0.039)\end{array}$ & $\begin{array}{c}0.718 * * * \\
(0.128) \\
1.010^{* *} \\
(0.396) \\
-0.093 * * \\
(0.036)\end{array}$ & $\begin{array}{c}0.716^{* * *} \\
(0.126) \\
0.961 * \\
(0.478) \\
-0.093 * * \\
(0.036)\end{array}$ \\
\hline $\begin{array}{l}\text { Educación y } \\
\text { Cultura }\end{array}$ & $\begin{array}{l}\text { Educación del padre } \\
\text { Log Gasto público per cápita } \\
\begin{array}{l}\text { Educación del padre } \times \text { Log Gasto público per } \\
\text { cápita }\end{array}\end{array}$ & $\begin{array}{c}0.864 * * * \\
(0.247) \\
0.997 * \\
(0.513) \\
-0.088^{*} \\
(0.047)\end{array}$ & $\begin{array}{c}0.804 * * * \\
(0.227) \\
1.392 * * \\
(0.511) \\
-0.078 * \\
(0.044)\end{array}$ & $\begin{array}{c}0.820^{* * *} \\
(0.226) \\
1.043^{*} \\
(0.581) \\
-0.081^{*} \\
(0.043)\end{array}$ \\
\hline $\begin{array}{l}\text { Educación } \\
\text { básica }\end{array}$ & $\begin{array}{l}\text { Educación del padre } \\
\text { Log Gasto público per cápita } \\
\begin{array}{l}\text { Educación del padre } \times \text { Log Gasto público per } \\
\text { cápita }\end{array}\end{array}$ & $\begin{array}{c}0.607 * * * \\
(0.168) \\
0.553 \\
(0.358) \\
-0.043 \\
(0.035)\end{array}$ & $\begin{array}{c}0.547 * * * \\
(0.153) \\
0.928 * * \\
(0.398) \\
-0.032 \\
(0.032)\end{array}$ & $\begin{array}{c}0.567 * * * \\
(0.157) \\
0.658 \\
(0.491) \\
-0.036 \\
(0.032)\end{array}$ \\
\hline $\begin{array}{l}\text { Educación } \\
\text { superior y } \\
\text { universitaria }\end{array}$ & $\begin{array}{l}\text { Educación del padre } \\
\text { Log Gasto público per cápita } \\
\begin{array}{l}\text { Educación del padre } \times \text { Log Gasto público per } \\
\text { cápita }\end{array}\end{array}$ & $\begin{array}{c}0.502 * * * \\
(0.083) \\
0.631 * \\
(0.341) \\
-0.045 \\
(0.032)\end{array}$ & $\begin{array}{c}0.497 * * * \\
(0.082) \\
0.628 \\
(0.430) \\
-0.043 \\
(0.032)\end{array}$ & $\begin{array}{c}0.490 * * * \\
(0.082) \\
0.485 \\
(0.425) \\
-0.041 \\
(0.032)\end{array}$ \\
\hline $\begin{array}{l}\text { Vivienda y } \\
\text { Urbanismo }\end{array}$ & $\begin{array}{l}\text { Educación del padre } \\
\text { Log Gasto público per cápita } \\
\begin{array}{l}\text { Educación del padre } \times \text { Log Gasto público per } \\
\text { cápita }\end{array}\end{array}$ & $\begin{array}{c}0.325^{* * *} \\
(0.043) \\
0.103 \\
(0.234) \\
0.019 \\
(0.013)\end{array}$ & $\begin{array}{c}0.325^{* * *} \\
(0.039) \\
0.292 \\
(0.272) \\
0.018 \\
(0.013)\end{array}$ & $\begin{array}{c}0.329 * * * \\
(0.039) \\
0.278 \\
(0.376) \\
0.017 \\
(0.013)\end{array}$ \\
\hline $\begin{array}{l}\text { Agua y } \\
\text { alcantarillado }\end{array}$ & $\begin{array}{l}\text { Educación del padre } \\
\text { Log Gasto público per cápita } \\
\begin{array}{l}\text { Educación del padre } \times \text { Log Gasto público per } \\
\text { cápita }\end{array}\end{array}$ & $\begin{array}{c}0.350 * * * \\
(0.029) \\
-0.287 * * \\
(0.111) \\
0.016 \\
(0.010)\end{array}$ & $\begin{array}{c}0.348 * * * \\
(0.030) \\
-0.275 * * * \\
(0.097) \\
0.017 \\
(0.010)\end{array}$ & $\begin{array}{c}0.347 * * * \\
(0.029) \\
-0.246^{* *} \\
(0.109) \\
0.017 \\
(0.010)\end{array}$ \\
\hline
\end{tabular}

Fuente: Elaboración propia sobre la base de varias fuentes de información. Ver la sección de datos para más detalles.

Nota: $* * * p$-value $<0.01, * *$-value $<0.05, * p$-value $<0.1$. Entre paréntesis se reportan los errores estándares agrupados a nivel provincial. Ver la nota del Cuadro 12 con la descripción de la especificación de cada modelo. 
Gráfico A1. Participación promedio del Gasto público provincial en el Gasto público consolidado total y social según categorías. 1998-2008.

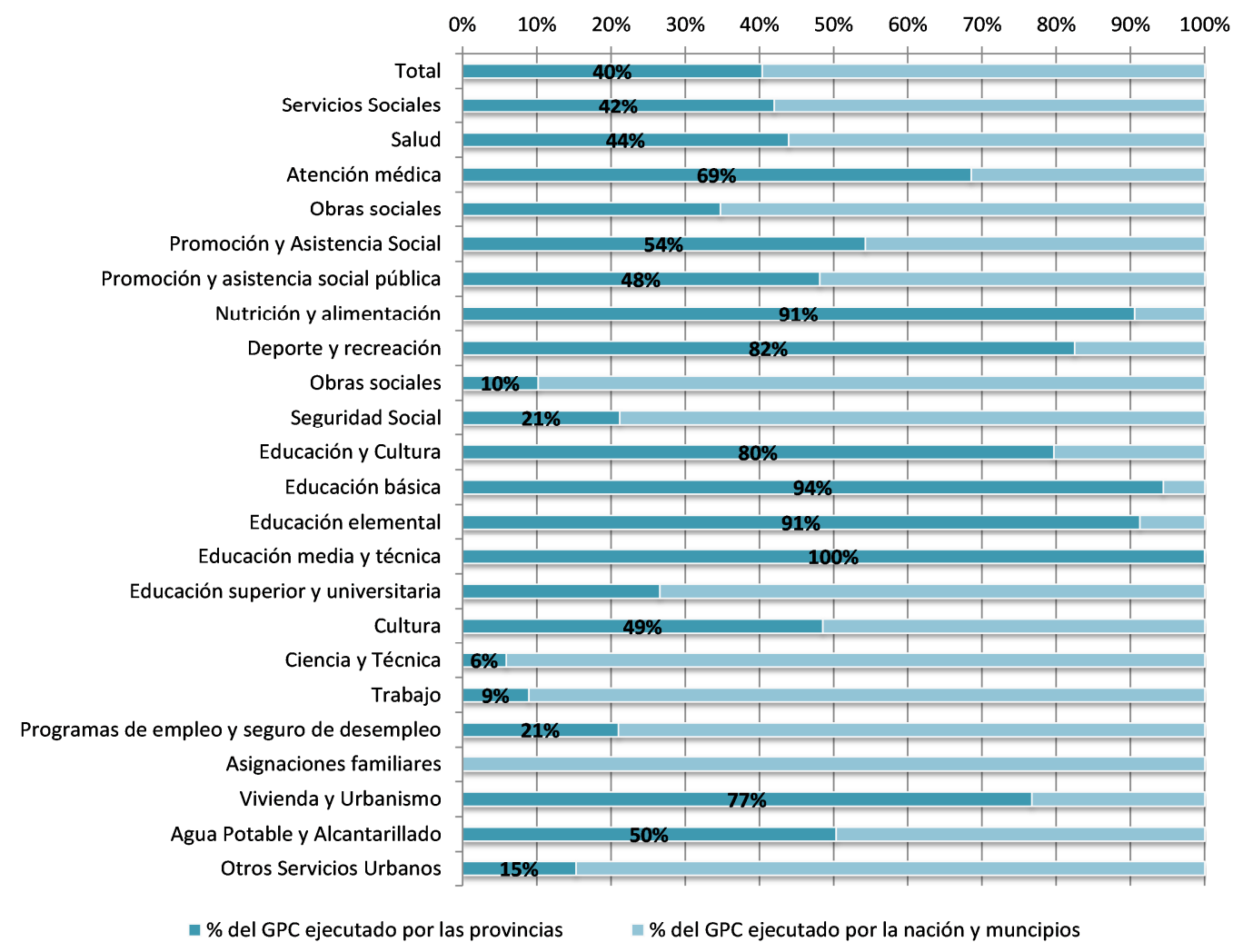

Fuente: Elaboración propia sobre la base de DAGPyPS y MECON. 